Universidade de São Paulo

Instituto de Física

\title{
Teorias de Calibre à Temperatura Finita e a Equação de Boltzmann
}

\author{
Renan Buosi Ferreira
}

Orientador: Prof. Dr. Fernando Tadeu Caldeira Brandt

Dissertação de mestrado apresentada ao Instituto de Física visando a obtenção do título de Mestre em Ciências

Banca Examinadora:

Prof. Dr. Fernando Tadeu Caldeira Brandt (IF-USP)

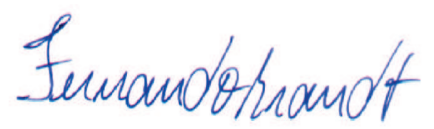

Prof. Dr. Josif Frenkel (IF-USP)

Prof. Dr. Bruto Max Pimentel (IFT-UNESP)

São Paulo

2015 
FICHA CATALOGRÁFICA

Preparada pelo Serviço de Biblioteca e Informação do Instituto de Física da Universidade de São Paulo

Ferreira, Renan Buosi

Teorias de calibre à temperatura finita e a equação de Boltzmann. São Paulo, 2015.

Dissertação (Mestrado) - Universidade de São Paulo. Instituto de Física. Depto. de Física Experimental.

Orientador: Prof. Dr. Fernando Tadeu Caldeira Brandt

Área de Concentração: Física de Partícula Elementares.

Unitermos: 1. Física de partículas; 2. Teoria quântica de campos; 3. Física térmica.

USP/IF/SBI-085/2015 


\section{Agradecimentos}

Não é por acaso que iniciarei agradecendo ao Professor Fernando, cuja dedicação e paciência foram enormes, e sem as quais não conseguiria enfrentar os desafios ao longo desses meses.

Gostaria de agradecer também aos meus pais, que sempre me apoiaram em qualquer empreitada na qual eu me introduzi, e obviamente, por terem provido todo o suporte que necessitei para chegar à presente singularidade de minha vida.

Além disso, não posso esquecer de prestar minha gratidão à INFRAERO, que além de permitir meu auto-sustento, me deu condições para que pudesse desenvolver minhas atividades acadêmicas. E como toda empresa é composta por pessoas, gostaria de agradecer em especial aos meus gestores(Daniel, Aderlei, Myron e Mário) pela compreensão e apoio despendidos durante todo esse tempo.

Quanto aos meus amigos, gostaria de agradecê-los um por um, mas o contexto me impede, e portanto, registro aqui o meu agradecimento ao Ulisses por ser um grande amigo, e ao Samuel, que muito me ajudou quando a vida profissional e acadêmica conflitaram.

Por fim, deixo essas últimas palavras de gratidão à minha parceira na vida, Marília, que me dá apoio incondicional, e me força a sempre ir em frente, mesmo quando o cansaço e o desânimo me abatem. Honestamente, com você tudo faz mais sentido. 



\section{Resumo}

A equivalência entre o formalismo de equação de transporte de Boltzmann e o limite de altas temperaturas da teoria de campos à temperatura finita é investigada no contexto das teorias de calibre. Essa conexão é feita através da comparação direta entre as amplitudes térmicas obtidas via a equação de transporte, sem termo de colisão, com aquelas resultantes do limite HTL das funções de Green térmicas em ordem de um loop. Para o formalismo quântico, partimos de um ensemble em equilíbrio, cujos efeitos térmicos são descritos via formalismo do tempo imaginário. Isso permite expressar as funções de Green térmicas como uma média estatística de amplitudes frontais (após continuação analítica). Já para o caso do formalismo clássico, combinamos as equações de Wong com a variação temporal da função de distribuição de partículas no espaço de fase. A equação resultante pode ser resolvida iterativamente, o que permite obter as várias ordens de aproximação para a corrente e as respectivas amplitudes térmicas. Finalmente, comparando as amplitudes obtidas a partir dos dois formalismos, pudemos verificar a sua equivalência. Ademais, apresentamos cálculos explícitos até segunda ordem de aproximação no caso de uma teoria não abeliana, e até quarta ordem para uma teoria abeliana, quando a distribuição de cargas é não neutra. 



\section{Abstract}

The equivalence between the formalism of Boltzmann transport equation and the high temperature limit of thermal field theory is investigated in the context of gauge theories. This connection is made through a direct comparison between the thermal amplitudes obtained via the collisionless transport equation with those resulting from the HTL limit of one loop thermal Green's function. For the quantum formalism we start with an ensemble in equilibrium, whose thermal effects are described by the imaginary time formalism. This allows one to write the thermal Green functions as a statistical average of forward scattering amplitudes (after analytic continuation). For the classical formalism, we combine Wong's equations with the time derivative of the particle distribution function in phase space. The resulting equation can be solved in an iterative fashion, yielding the perturbative results for the current and the respective thermal amplitudes. Finally, comparing the amplitudes obtained from the two formalisms, we were able to verify their equivalence. Moreover, we present explicit calculations for a non-Abelian theory up to the second order approximation, and in the case of an abelian theory we proceed up to fourth order, when the charge distribution is not neutral. 



\section{Lista de abreviaturas e siglas}

$\begin{array}{ll}\text { 1PI } & \text { One-particle irreducible } \\ \text { HTL } & \text { Hard Thermal Loop } \\ \text { QED } & \text { Quantum Electrodynamics } \\ \text { QCD } & \text { Quantum Chromodynamics } \\ \text { QFT } & \text { Quantum Field Theory } \\ \text { TQFT } & \text { Thermal Quantum Field Theory }\end{array}$





\section{Lista de ilustrações}

Figura 1 - (a) Polos de uma cotangente hiperbólica envoltos por caminhos de integração circulares; (b) Caminho de integração formado pela deformação dos caminhos circulares contidos em (a). . . . . . . . . . . . 30

Figura 2 - Ordem mais baixa dos diagramas de um loop com pernas externas, que devido ao seu formato é chamado de tadpole. . . . . . . . . . . . . . 33

Figura 3 - Diagrama de um loop com duas pernas externas para uma teoria $\phi^{3}$. 34

Figura 4 - Diagrama de um loop com $n$ pernas externas para uma teoria genérica. 35

Figura 5 - (a) Amplitude frontal obtida a partir de um diagrama com um loop e $n$ pernas externas; (b) permutação cíclica de (a) . . . . . . . . . . . . 38

Figura 6 - (a) Vértice de interação entre férmions e bósons ; (b) Vértice da autointeração de 3 pontos dos bósons; (c) Vértice da auto-interação de 4 pontos dos bósons. . . . . . . . . . . . . . . . . . 4 45

Figura 7 - Compontes da auto-energia dos bósons de calibre, cujas interações associadas são: (a) auto-interação de 4 pontos dos bósons de calibre; (b) auto-interação de 3 pontos dos bósons de calibre ; (c) interação entre bósons de calibre e férmions; e (d) interação entre bósons de calibre e ghosts.

Figura 8 - Amplitudes frontais, com auto-interações de 3 pontos, da auto-energia dos bósons de calibre.

Figura 9 - Amplitudes frontais, com interações entre bósons de calibre e ghosts, da auto-energia dos bósons de calibre.

Figura 10 - Amplitudes frontais, com interações entre bósons de calibre e férmions, da auto-energia dos bósons de calibre. . . . . . . . . . . . . 5 56

Figura 11 - Auto-energia dos férmions. . . . . . . . . . . . . . . 57

Figura 12 - Amplitudes frontais, com interações entre bósons de calibre e férmions, da auto-energia dos férmions. . . . . . . . . . . . . . . . . 57

Figura 13 - Auto-energia dos ghosts. . . . . . . . . . . . . . 58

Figura 14 - Amplitudes frontais, com interações entre bósons de calibre e ghosts, da auto-energia dos ghosts. . . . . . . . . . . . . . . . . . . . 59

Figura 15 - Diagrama de um loop fermiônico com $n$ fótons externos. . . . . . . . . 82

Figura 16 - Amplitude frontal de um férmion térmico interagindo com $n$ fótons externos. . . . . . . . . . . . . . . . . . . . . 9 91

Figura 17 - Dependência das amplitudes térmicas em relação ao parâmetro $\bar{m}=\frac{m}{T}$,

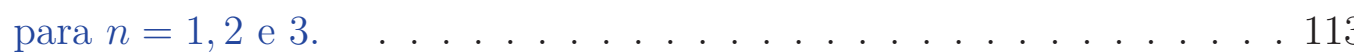

Figura 18 - Dependência das amplitudes térmicas em relação ao parâmetro $\bar{m}=\frac{m}{T}$, para $n=4 \ldots \ldots \ldots \ldots \ldots 114 \ldots \ldots \ldots$ 
Figura 19 - Fluxograma do algoritmo utilizado no cálculo do limite de altas temperaturas das correções do propagador de um fóton, em um plasma no âmbito da QED. . . . . . . . . . . . . . . . . . . . . 120

Figura 20 - Fluxograma do algoritmo utilizado nos cálculos das amplitudes térmicas via iterações da equação de transporte de Boltzmann, para um plasma eletrônico. . . . . . . . . . . . . . . . . . . . . . . 122

Figura 21 - Cálculos efetuados via o aplicativo Mathematica para uma amplitude térmica com 3 pernas externas seguindo o algoritmo da figura 19, onde os comandos, texto em azul, estão seguidos por seus respectivos outputs.124

Figura 22 - Cálculos efetuados via o aplicativo Mathematica para uma amplitude térmica com 4 pernas externas seguindo o algoritmo da figura 19, onde os comandos, texto em azul, estão seguidos por seus respectivos outputs.125

Figura 23 - Cálculos efetuados via o aplicativo Mathematica para uma amplitude térmica com 3 pernas externas seguindo o algoritmo da figura 20, onde os comandos, texto em azul, estão seguidos por seus respectivos outputs. 126

Figura 24 - Cálculos efetuados via o aplicativo Mathematica para uma amplitude térmica com 4 pernas externas seguindo o algoritmo da figura 20, onde os comandos, texto em azul, estão seguidos por seus respectivos outputs.127

Figura 25 - Resultado dos cálculos via o aplicativo Mathematica para uma amplitude térmica com 3 pernas externas, onde os comandos, texto em azul, estão seguidos pelos outputs. . . . . . . . . . . . . . . . . . 127

Figura 26 - Resultado dos cálculos via o aplicativo Mathematica para uma amplitude térmica com 4 pernas externas, onde os comandos, texto em azul, estão seguidos pelos outputs. . . . . . . . . . . . . . . . . . . . 127 


\section{Sumário}

INTRODUÇÃo $\ldots \ldots \ldots \ldots \ldots \ldots \ldots \ldots$

2 TEORIA QUÂNTICA DE CAMPOS À TEMPERATURA FINITA . 17

$2.1 \quad$ Formalismo do tempo imaginário . . . . . . . . . . 17

$2.2 \quad$ Regras de Feynman à temperatura finita . . . . . . . . . . 21

$2.3 \quad$ Amplitudes frontais . . . . . . . . . . . . . . . 29

2.3.1 Amplitudes frontais para a teoria $\phi^{3} \ldots \ldots \ldots \ldots . \ldots . \ldots 32$

2.3.2 Amplitudes frontais para uma teoria genérica . . . . . . . . . . 35

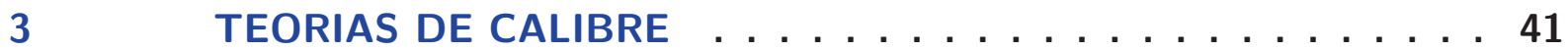

3.1 Transformações de calibre . . . . . . . . . . . . . 42

3.2 Regras de Feynman para as teorias de calibre . . . . . . . . . 46

3.2.1 Método de Faddeev-Popov . . . . . . . . . . . . . . . . 48

3.2.2 Interações na teoria de Yang-Mills . . . . . . . . . . . . . . 50

3.3 Amplitudes frontais da teoria de Yang-Mills . . . . . . . . . . 52

3.3.1 Auto-energias da teoria de Yang-Mills . . . . . . . . . . . . . 53

4 LIMITE DE ALTAS TEMPERATURAS DA TQFT . . . . . . . . 61

$4.1 \quad$ Limite de altas temperaturas para a teoria $\lambda \phi^{3} \ldots \ldots$

4.2 Limite de altas temperaturas para a teoria de Yang-Mills . . . . 64

4.2.1 HTL aplicado à auto-energia dos bósons de calibre . . . . . . . . . . 64

4.2.1.1 Decomposição tensorial das auto-energias . . . . . . . . . . . . . . 66

$4.2 .1 .2 \quad$ Integrações . . . . . . . . . . . . . . . . . 67

4.2.2 HTL aplicado à auto-energia dos ghosts . . . . . . . . . . . 70

4.2.3 HTL aplicado à auto-energia dos férmions . . . . . . . . . . . . . 71

5 EQUAÇÃO DE TRANSPORTE DE BOLTZMANN $\ldots \ldots \ldots 73$

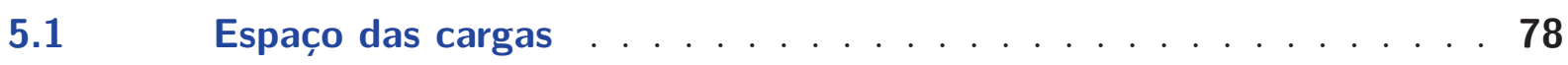

5.2 Integração no espaço dos momentos . . . . . . . . . 79

6 LIMITE DE ALTAS TEMPERATURAS DA QED E A EQUAÇÃO DE TRANSPORTE . . . . . . . . . . . . . . 81

6.1 Equação de Boltzmann . . . . . . . . . . . . . . 82

6.2 Limite de altas temperaturas da QED . . . . . . . . . . . . 89

$7 \quad$ CONCLUSÃO $\ldots \ldots \ldots \ldots \ldots$ 
APÊNDICE A - AÇÃO EFETIVA . . . . . . . . . . 99

A.1 Funcional gerador das funções de correlação . . . . . . . . . . 103

APÊNDICE B - PROPAGADORES NO ENSEMBLE COMPLETO 105

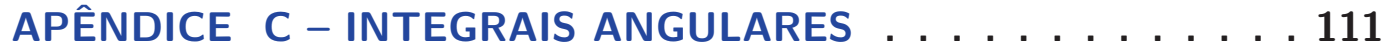

APÊNDICE D - FUNÇÃO TÉRMICA NO LIMITE DE MASSA NULA . . . . . . . . . . . . 113

APÊNDICE E - ALGORITMO DO CÁLCULO DAS AMPLITUDES TÉRMICAS . . . . . . . . . . . . . 117

E.1

QED . . . . . . . . . . . . . . . . . . . 117

E.2 Equação de transporte de Boltzmann . . . . . . . . . . . . . 120

E.3 Comparação entre os resultados . . . . . . . . . . . . . . 122

Referências . . . . . . . . . . . . . . . . . . 129 


\section{Introdução}

O presente trabalho é composto de um estudo acerca da conexão entre a equação de transporte de Boltzmann, que é desenvolvida em um contexto clássico, e o limite de altas temperaturas da QFT $^{1}$ (BRANDT; FERREIRA; THUORST, 2015; LITIM; MANUEL, 2002; BRANDT; FRENKEL; TAYLOR, 1995a). Com isso, além de analisar em quais limites as teorias quânticas e clássicas são equivalentes, iremos também descrever um procedimento que a priori pode ser aplicado na extensão da teoria clássica, já que ao obtermos o modelo de um sistema no contexto quântico, poderemos encontrar seu limite clássico.

Desta forma, um dos conceitos cruciais a serem trabalhados é o da temperatura, que sempre surge ao lidarmos com sistemas de muitos corpos. Adicionalmente, quando os fenômenos envolverem a física de altas energias, isto é, quando os efeitos relativísticos e quânticos são significativos, deve-se utilizar a QFT juntamente com a mecânica estatística.

Dentre os vários sistemas em que ocorre essa dinâmica, podemos citar as anãs brancas, que são estrelas compostas por um gás degenerado de elétrons relativísticos, ou por exemplo, as colisões entre partículas com altas energias, que formam um plasma de quarks e glúons. Assim, fica evidente que a QFT à temperatura finita pode ser empregada em uma gama muito grande de fenômenos físicos, variando desde aqueles na escala do micro (dinâmica de nucleons) até os na escala do macro (cosmologia).

Para dar início a este estudo, apresentaremos no capítulo 2 o formalismo do tempo imaginário, que introduz os efeitos da temperatura na QFT, e tem como consequência a discretização da energia, cujos valores permitidos serão representados pelas frequências de Matsubara (MATSUBARA, 1955). Sendo assim, em vez de efetuarmos as integrais nas energias considerando um intervalo contínuo e infinito, assim como é feito na QFT usual, lidaremos com somatórias, que após uma sequência de manipulações, serão escritas em função de integrais em um plano complexo.

Ainda no capítulo 2, veremos que as amplitudes térmicas(DAS, 1997; BELLAC, 1996), advindas de certos diagramas de Feynman, em ordem de um loop, podem ser escritas como uma média estatística de amplitudes frontais, que por sua vez são representadas por diagramas em ordem de árvore. Consequentemente, por utilizarmos essa linguagem diagramática (teoria de perturbação), serão descritas também as regras de Feynman à temperatura finita(KAPUSTA; GALE, 2006).

Já no capítulo seguinte, além de definir as transformações de calibre associadas ao

1 Teoria Quântica de Campos 
grupo de Lie SU(N)2 , apresentaremos a construção da teoria de Yang-Mills(YANG; MILLS, 1954; PESKIN; SCHROEDER, 1995) através da invariância sob essas transformações. Ademais, como a lagrangiana para esta teoria possui graus de liberdade não físicos, utilizaremos o método de Faddeev-Popov(FADDEEV; POPOV, 1967) para introduzir ghosts, e obter as regras de Feynman para teorias de calibre. A partir deste ponto, aplicaremos essas regras para obter as auto-energias dos bósons de calibre, dos férmions e dos ghosts, pois como demonstraremos neste mesmo capítulo, essas funções juntamente com as outras amplitudes térmicas geradas por diagramas 1PI formam as correções para os propagadores destes campos.

Para concluir o formalismo quântico, no capítulo 4 introduziremos o limite HTL (BRAATEN; PISARSKI, 1990), que é equivalente ao limite de altas temperaturas, o qual será aplicado à teoria $\lambda \phi^{4}$ para fins de exemplificação. Posteriormente, estudaremos detalhadamente este mesmo limite aplicado à teoria de Yang-Mills(FRENKEL; TAYLOR, 1990; TAYLOR; WONG, 1990), culminando em um resultado explícito para as amplitudes térmicas.

Complementarmente à esses cálculos para a TQFT ${ }^{3}$, no capítulo 5 será apresentado o formalismo da equação de transporte de Boltzmann para um plasma não-abeliano, já que esse sistema físico equivale ao limite clássico da teoria de Yang-Mills. Como veremos, esse formalismo parte das equações de movimento de Wong resultando em uma equação de transporte iterativa, que permitirá obter sucessivas ordens de aproximação.

Ao compararmos os resultados obtidos nos capítulos 4 e 5, concluiremos que ambos os formalismos são equivalentes para a teoria de Yang-Mills até a segunda ordem de aproximação. Já para ordens superiores, seguiremos no capítulo 6 com uma teoria de calibre abeliana, mais especificamente a $\mathrm{QED}^{4}$, para qual efetuaremos cálculos até a quarta ordem de aproximação(BRANDT; FERREIRA; THUORST, 2015). Essa escolha foi motivada pelo fato da QED possuir uma dependência térmica diferente para cada uma das ordens de aproximação, o que não ocorre para a $\mathrm{QCD}^{5}$. Além disso, neste capítulo consideraremos um plasma eletrônico com uma distribuição de cargas não neutra, o que nos permitirá demonstrar uma conexão ainda mais profunda entre esses dois formalismos.

Em todo o trabalho utilizaremos unidades tais que $\hbar=c=k_{B}=1$.

2 Grupo especial unitário de grau N, com dimensão $N^{2}-1$.

3 Teoria Quântica de Campos Térmica

4 Eletrodinâmica Quântica

5 Cromodinâmica Quântica 


\section{Teoria quântica de campos à temperatura finita}

Ao longo dos anos foram criados três formalismos que incluem os efeitos da temperatura na QFT. O primeiro deles, chamado de formalismo do tempo imaginário é o mais adequado para descrever sistemas em equilíbrio térmico, embora seja possível estendê-lo para sistemas com evolução temporal através de uma continuação analítica. Já nos outros dois formalismos, os efeitos da temperatura são introduzidos via condições de contorno no caminho da integração temporal, sendo que estes são denominados como trajetória temporal fechada(SCHWINGER, 1961), e dinâmica de campos térmicos (UMEZAWA; MATSUMOTO; TACHIKI, 1982).

Desta forma, para os fenômenos aqui estudados, o formalismo mais apropriado é o do tempo imaginário, pois como veremos, ele permite que utilizemos as técnicas já desenvolvidas para a QFT à temperatura nula com apenas algumas modificações.

\subsection{Formalismo do tempo imaginário}

Ao considerarmos um sistema de várias partículas, no âmbito da mecânica estatística, em contato com um reservatório, que permite a troca de calor e partículas, é conveniente utilizar o Ensemble Grand Canônico dentre todos os existentes, pois nele a temperatura $T$, o volume $V$ e o potencial químico $\mu$ são constantes. Além disso, podemos associar a este ensemble uma matriz densidade de probabilidade, que defini-se como:

$$
\hat{\rho}=e^{-\beta\left(\hat{H}-\mu_{i} \hat{N}_{i}\right)}
$$

em que $\beta$ é o inverso da temperatura, $\hat{H}$ é o operador hamiltoniano e $\mu_{i}$ é o potencial químico associado ao operador $\hat{N}_{i}$, que representa a quantidade de partículas de uma certa espécie.

A escolha deste ensemble é também a mais adequada para descrever fenômenos físicos no âmbito da QFT à temperatura finita, pois além de permitir estudar os casos em equilíbrio térmico, ele engloba a criação e aniquilação de partículas, que surge ao considerarmos os efeitos relativísticos.

Ademais, com o auxílio de (2.1) é possível definir a média de um operador $\hat{O}$, considerando as flutuações estatísticas,

$$
\langle\hat{O}\rangle=\frac{\operatorname{Tr}\left[e^{-\beta\left(\hat{H}-\mu_{i} \hat{N}_{i}\right)} \hat{O}\right]}{\operatorname{Tr}\left[e^{-\beta\left(\hat{H}-\mu_{i} \hat{N}_{i}\right)}\right]},
$$


sendo $\operatorname{Tr}$ a operação de traço.

A expressão acima possui uma importante função em seu denominador, a saber a função de partição $Z=\operatorname{Tr}[\hat{\rho}]$, que caracteriza o sistema físico em questão, pois através dela podemos calcular quantidades termodinâmicas tais como a energia $E$, a entropia $S$, a pressão $P$ e o número de partículas $N$ do sistema, conforme as seguintes relações termodinâmicas

$$
\begin{array}{r}
P=\frac{\partial(T \ln (Z))}{\partial V} \\
S=\frac{\partial(T \ln (Z))}{\partial T} \\
N_{i}=\frac{\partial(T \ln (Z))}{\partial \mu_{i}} \\
E=T S-P V+\mu_{i} N_{i}
\end{array}
$$

Portanto, o cálculo da função de partição é essencial para a descrição de um sistema de muitos corpos. Logo, este será o nosso objetivo nos desenvolvimentos a seguir. Para isto, consideraremos os casos em que é possível encontrar funções de estado $\left|\phi_{a}\right\rangle$ definidas como autovetores da hamiltoniana, assim permitindo o cálculo do traço da seguinte forma:

$$
Z(\beta)=\sum_{a} \int d \phi_{a}\left\langle\phi_{a}\left|e^{-\beta\left(\hat{H}-\mu_{i} \hat{N}_{i}\right)}\right| \phi_{a}\right\rangle
$$

Agora, diferentemente do que é feito na mecânica estatística usual, que utiliza funções de estado dependentes da posição $x$ e do momento $p$ associados às partículas, utilizaremos funções que variam com um campo $\phi$ e seu momento conjugado $\pi$. Esses dois campos, $\phi$ e $\pi$, estão definidos para todo o espaço, e por isto, para contabilizar todas as suas contribuições, as relações a seguir virão acompanhadas de integrais do tipo $\int d^{3} x$.

Além disso, nos próximos desenvolvimentos não consideraremos os operadores $\hat{N}_{i}$, pois eles são necessários apenas nos casos em que a densidade de partículas é finita, e o enfoque do presente trabalho limita-se aos casos para os quais a temperatura é finita, independentemente da densidade de partículas.

A fim de introduzir efeitos quânticos na expressão (2.7), seguiremos os mesmos passos feitos para a QFT usual (FEYNMAN; HIBBS, 1965), quando tratada via integrais de trajetória. Posto isto, analisaremos o efeito de sucessivas transformações, que isoladamente evoluem no tempo um determinado estado para outro muito próximo. Essa sequência se dará praticamente de forma contínua, sendo que ao final retornaremos às mesmas configurações do estado inicial. Assim, iniciaremos dividindo a matriz densidade de probabilidade em várias outras matrizes contendo transformações infinitesimais,

$$
\left\langle\phi_{a}\left|e^{-\beta \hat{H}}\right| \phi_{a}\right\rangle=\left\langle\phi_{a}\left|e^{-\Delta \tau \hat{H}} \ldots e^{-\Delta \tau \hat{H}}\right| \phi_{a}\right\rangle
$$


Nesta relação definimos $\Delta \tau=\frac{\beta}{N}, \operatorname{com} N \rightarrow \infty$. Agora, ao considerar que o problema físico em questão admite soluções que formem uma base completa, estabeleceremos relações de completeza tanto para $|\phi\rangle$ quanto para a função de estado no espaço dos momentos conjugados $|\pi\rangle$.

$$
\begin{aligned}
& \int d \phi_{i}\left|\phi_{i}\right\rangle\left\langle\phi_{i}\right|=1 \\
& \int \frac{d \pi_{i}}{2 \pi}\left|\pi_{i}\right\rangle\left\langle\pi_{i}\right|=1
\end{aligned}
$$

Intercalando estas relações de completeza entre as exponenciais presentes em (2.8), temos:

$$
\begin{array}{r}
\left\langle\phi_{a}\left|e^{-\beta \hat{H}}\right| \phi_{a}\right\rangle=\lim _{N \rightarrow \infty} \int\left(\prod_{i=1}^{N} \frac{d \pi_{i}}{2 \pi} d \phi_{i}\right)\left\langle\phi_{a} \mid \pi_{N}\right\rangle\left\langle\pi_{N}\left|e^{-\Delta \tau \hat{H}}\right| \phi_{N}\right\rangle\left\langle\phi_{N} \mid \pi_{N-1}\right\rangle \ldots \\
\ldots\left\langle\phi_{2} \mid \pi_{1}\right\rangle\left\langle\pi_{1}\left|e^{-\Delta \tau \hat{H}}\right| \phi_{1}\right\rangle\left\langle\phi_{1} \mid \phi_{a}\right\rangle .
\end{array}
$$

Além das relações (2.9) e (2.10), ainda utilizaremos a definição do produto entre as funções de estado $|\phi\rangle$ e $|\pi\rangle$,

$$
\left\langle\phi_{i} \mid \pi_{i}\right\rangle=e^{i \int d^{3} x \pi_{i}(x) \phi_{i}(x)}
$$

que somadas ao limite $\Delta \tau \rightarrow 0$, nos permitirão obter para as exponenciais em (2.11) a seguinte expansão:

$$
\left\langle\pi_{i}\left|e^{-\Delta \tau \hat{H}}\right| \phi_{i}\right\rangle \approx\left\langle\pi_{i}|(1-\Delta \tau \hat{H})| \phi_{i}\right\rangle=\left(1-\Delta \tau H_{i}\right) e^{-i \int d^{3} x \pi_{i}(x) \phi_{i}(x)},
$$

em que, $H_{i}=\int d^{3} x \mathcal{H}\left(\pi_{i}(x), \phi_{i}(x)\right)$, sendo $\mathcal{H}$ a densidade de hamiltoniana.

Como o sistema irá retornar ao estado inicial no término da evolução temporal, devemos impor $\phi_{N+1}=\phi_{1}=\phi_{a}$, e assim, poderemos facilmente calcular a integral em $\phi_{1}$ na expressão (2.11), pois $\left\langle\phi_{1} \mid \phi_{a}\right\rangle=\delta\left(\phi_{1}-\phi_{a}\right)$. A substituição de (2.13) e (2.12) em (2.11) resultará em

$$
\left\langle\phi_{a}\left|e^{-\beta \hat{H}}\right| \phi_{a}\right\rangle=\lim _{N \rightarrow \infty} \int\left(\prod_{i=1}^{N} \frac{d \pi_{i}}{2 \pi} d \phi_{i}\right) \delta\left(\phi_{1}-\phi_{a}\right) e^{-\Delta \tau \sum_{j=1}^{N} \int d^{3} x\left[\mathcal{H}\left(\pi_{j}(x), \phi_{j}(x)\right)-i \pi_{j}(x) \frac{\phi_{j+1}(x)-\phi_{j}(x)}{\Delta \tau}\right]} .
$$

Por fim, ao impor os limites $N \rightarrow \infty$ e $\Delta \tau \rightarrow 0$, as transformações presentes em (2.14) passarão a ser contínuas.

$$
\left\langle\phi_{a}\left|e^{-\beta \hat{H}}\right| \phi_{a}\right\rangle=\int[d \pi] \int_{\phi(x, 0)}^{\phi(x, \beta)}[d \phi] e^{\int^{0} d \tau \int d^{3} x\left[i \pi(x, \tau) \frac{\partial \phi(x, \tau)}{\partial \tau}-\mathcal{H}(\pi(x, \tau), \phi(x, \tau))\right]}
$$


Estas medidas de integração $[d \pi]$ e $[d \phi]$ são apenas representações simbólicas das sequências de integrações funcionais definidas em (2.14). Adicionalmente, é necessário recordar que a integral no campo $\phi$ é feita em um intervalo periódico já que $\phi(x, 0)=$ $\phi(x, \beta)$.

Ademais, esta equação (2.15), como será demonstrado nos seguintes parágrafos, nos diz que uma teoria de campos com $D+1$ dimensões espaço-temporais descreve um sistema quântico térmico com $D$ dimensões espaciais. Além disto, veremos também que a variável temporal é sacrificada em favor da inclusão da temperatura, o que torna este formalismo adequado aos sistemas em equilíbrio térmico.

Até agora, todas as passagens reproduzidas nesta seção são muito semelhantes àquelas efetuadas em livros introdutórios à QFT (PESKIN; SCHROEDER, 1995) no que diz respeito ao cálculo das amplitudes de transição via integrais de trajetória. Porém existem algumas diferenças entre (2.15) e a expressão das amplitudes de transição entre dois estados formando um ciclo periódico (KAPUSTA; GALE, 2006), que pode ser escrita como

$$
\left\langle\phi_{a}\left|e^{-i H t_{f}}\right| \phi_{a}\right\rangle=\int[d \pi] \int_{\phi(x, 0)}^{\phi\left(x, t_{f}\right)}[d \phi] e^{i \int_{0}^{t_{f}} d t \int d^{3} x\left[\pi(x, t) \frac{\partial \phi(x, t)}{\partial t}-\mathcal{H}(\pi(x, t), \phi(x, t))\right]}
$$

na qual $t$ é a variável temporal pertencente ao espaço de Minkowski e $t_{f}$ é o tempo de duração da transição.

Ao compararmos as expressões (2.16) e (2.15), nota-se que é possível obter a função de partição a partir das amplitudes de transição, se aplicada uma rotação de Wick do tipo $t \rightarrow-i \tau$. Desta forma, fica claro que poderemos aproveitar todo o formalismo desenvolvido para as amplitudes de transição à temperatura nula, necessitando apenas considerar as consequências advindas da rotação de Wick, já que essa transformação temporal no plano complexo nos leva de um espaço de Minkowski para um euclidiano.

Em consequência à essa mudança na parte temporal da métrica espacial, teremos também uma mudança na parte temporal do quadrimomento, pois como os campos são periódicos em $\tau$, que é restrito ao intervalo $0 \leq \tau \leq \beta$, a componente do quadrimomento conjugada à $\tau$ deverá ser discreta. Portanto, a expansão em série de Fourier dos campos $\phi$ tem a seguinte forma:

$$
\phi(x, \tau)=\left(T L^{3}\right)^{-\frac{1}{2}} \sum_{n=-\infty}^{\infty} \int d^{3} p e^{i\left(\vec{p} \cdot \vec{x}+\omega_{n} \tau\right)} \phi_{n}\left(\omega_{n}, \vec{p}\right),
$$

em que $L$ é a dimensão de cada componente espacial, o que implica $L \rightarrow \infty$.

Ao substituir (2.17) em $\phi(x, \beta)=\phi(x, 0)$, concluímos que $\omega_{n}^{B}=2 \pi n T$, sendo $n$ um número inteiro. Estas variáveis $\omega_{n}^{B}$ são citadas na literatura como as frequências de Matsubara, e possuem estes valores apenas para partículas com estatística bosônica. 
Em contrapartida, as partículas com estatística fermiônica obedecem relações de antiperiodicidade, $\phi^{F}(x, \beta)=-\phi^{F}(x, 0)$, e consequentemente, ao substituirmos as expansões em série de Fourier dos campos $\phi^{F}$ nesta relação, obteremos $\omega_{n}^{F}=2(n+1) \pi T$ para as frequências de Matsubara.

Caso estivéssemos lidando com a QFT à temperatura nula, a expressão (2.17) conteria $p_{0} t$ ao invés de $\omega_{n} \tau$, e como a rotação de Wick transforma $t$ em $-i \tau$, para que seja mantida a equivalência entre os dois formalismos, a energia deverá obedecer a seguinte rotação de Wick $p_{0} \rightarrow i \omega_{n}$.

Por fim, a expressão (2.15) será o ponto de partida para o desenvolvimento das regras de Feynman na seção seguinte, porém, antes de fazê-lo, é importante notar que no caso de $\beta \rightarrow \infty$, essa expressão se reduz a teoria de campos usual definida em um espaço euclidiano. Por outro lado, no caso de $\beta \rightarrow 0$, que é o limite de altas temperaturas, iremos nos aproximar de um sistema clássico. Este último caso será amplamente explorado ao compararmos os resultados obtidos via QFT à temperatura finita com aqueles obtidos via formalismo clássico.

\subsection{Regras de Feynman à temperatura finita}

Os conceitos apresentados em (2.15) e (2.16) são passíveis de generalização para qualquer teoria, e suas aplicações se estendem desde as teorias mais simples, contendo apenas campos escalares, até as mais complexas. Todavia, não é possível obter uma solução fechada quando os termos de interação (não quadráticos) são levados em conta.

No entanto, quando os termos de interação são tratados como uma perturbação, podemos utilizar um método de expansão, a ser descrito a seguir, que culminará nas regras de Feynman. Embora este método seja amplamente utilizado em vários ramos da física, por exemplo, física de partículas e física do estado sólido, a convergência de sua expansão ainda não foi comprovada com o devido rigor matemático.

Por conveniência continuaremos a utilizar o campo escalar $\phi$, sendo que a generalização do método a ser apresentado, pode ser encontrada na literatura(RAMOND, 1990; MUTA, 1987; BIRRELL; DAVIES, 1982; ZEE, 2003). Para dar prosseguimento aos cálculos, será necessário explicitar a forma da densidade de lagrangiana ${ }^{1} \mathcal{L}$, que assumiremos ser:

$$
\mathcal{L}=\mathcal{L}_{0}-V_{i}=\frac{1}{2} \partial_{\mu} \phi(x, t) \partial^{\mu} \phi(x, t)-\frac{m^{2}}{2} \phi^{2}(x, t)-V_{I}
$$

Esta lagrangiana está definida no espaço de Minkowski, e após uma rotação de

1 No texto que está por vir, assim como ocorre na literatura, nos referenciaremos às densidades de lagrangiana por apenas lagrangiana. 
Wick será escrita como

$$
\mathcal{L}_{E}=-\frac{1}{2}\left(\partial_{\tau} \phi(x, \tau)\right)^{2}-\frac{1}{2}(\vec{\nabla} \phi(x, \tau))^{2}-\frac{m^{2}}{2} \phi(x, \tau)^{2}-V_{I} .
$$

A parte de interação desta lagrangiana, representada pelo potencial $V_{I}$, no caso mais geral de uma teoria renormalizável, irá conter termos proporcionais a $\phi^{3}$ ou a $\phi^{4}$. No entanto, para fins de simplificação e melhor compreensão do método, vamos considerar, na presente seção, apenas a interação $\lambda \phi^{4}$. Agora que todos os termos de $\mathcal{L}$ foram explicitados, o momento $\pi$ conjugado ao campo $\phi$ será:

$$
\pi(x, t)=\frac{\partial \mathcal{L}\left(\phi(x, t), \partial_{\mu} \phi(x, t)\right)}{\partial\left(\partial_{0} \phi(x, t)\right)}=\frac{\partial \phi(x, t)}{\partial t} \stackrel{\text { Rotação de Wick }}{\longrightarrow} i \frac{\partial \phi(x, \tau)}{\partial \tau} \equiv \pi(x, \tau) .
$$

Substituindo esse momento $\pi$ na transformação de Legendre que correlaciona a lagrangiana com a hamiltoniana, temos:

$$
\begin{aligned}
H=i \pi(x, \tau) \frac{\partial \phi(x, \tau)}{\partial \tau}-\mathcal{L}\left(\phi(x, \tau), \partial_{\mu} \phi(x, \tau)\right) & \\
= & \frac{\pi(x, \tau)^{2}}{2}+\frac{(\vec{\nabla} \phi(x, \tau))^{2}}{2}+\frac{m^{2} \phi^{2}(x, \tau)}{2}+\lambda \phi^{4}(x, \tau) .
\end{aligned}
$$

A relação (2.15) ilustra bem o resultado para a função de partição, porém a versão (2.14) com os campos discretizados é mais prática para efetuarmos os cálculos. Portanto, a função de partição respectiva à densidade de hamiltoniana (2.21) será

$$
\begin{aligned}
Z & =\lim _{N \rightarrow \infty} \prod_{i=1}^{N} \int \frac{d \pi_{i}}{2 \pi} \int_{\bigcirc} d \phi_{i} e^{-\Delta \tau \sum_{j=1}^{N} \int d^{3} x\left[\frac{\pi_{j}^{2}}{2}+\frac{\left(\vec{\nabla} \phi_{j}\right)^{2}}{2}+\frac{m^{2} \phi_{j}^{2}}{2}+\lambda \phi_{j}^{4}-i \pi_{j} \frac{\phi_{j+1}-\phi_{j}}{\Delta \tau}\right]} \\
& =\lim _{N \rightarrow \infty} \prod_{i=1}^{N} \int_{\bigcirc} d \phi_{i} e^{-\Delta \tau \sum_{j=1}^{N} \int d^{3} x\left[\frac{\left(\vec{\nabla} \phi_{j}\right)^{2}}{2}+\frac{m^{2} \phi_{j}^{2}}{2}+\lambda \phi_{j}^{4}\right]} \int \frac{d \pi_{i}}{2 \pi} e^{-\sum_{j=1}^{N} \int d^{3} x\left[\frac{\Delta \tau}{2} \pi_{j}^{2}-i\left(\phi_{j+1}-\phi_{j}\right) \pi_{j}\right]} .
\end{aligned}
$$

Na expressão acima, o símbolo $\bigcirc$ indica que o intervalo de integração é periódico. Nesta ação contida em (2.22), iremos dividir o espaço de configuração das posições em $M^{3}$ cubos infinitesimais com volume $L^{3}$. Além disto, a largura destes cubos estará condicionada a $L=a M, \operatorname{com} a \rightarrow 0$ e $M \rightarrow \infty$. Este procedimento juntamente com a mudança de variável $\pi_{j}=\frac{\Pi_{j}}{\sqrt{a^{3} \Delta \tau}}$, visam manter a adimensionalidade da função de partição.

Para cada um desses cubos haverá uma integral gaussiana do tipo

$$
\int_{-\infty}^{\infty} \frac{d \Pi_{j}}{2 \pi} e^{-\frac{1}{2} \Pi_{j}^{2}+i \sqrt{\frac{a^{3}}{\Delta \tau}}\left(\phi_{j+1}-\phi_{j}\right) \Pi_{j}}=\frac{e^{\frac{-a^{3}}{2 \Delta \tau}\left(\phi_{j+1}-\phi_{j}\right)^{2}}}{\sqrt{2 \pi}} .
$$


Substituindo este resultado em (2.22), teremos uma nova expressão para a função de partição que depende apenas do campo $\phi$.

$$
Z=\lim _{M, N \rightarrow \infty}(2 \pi)^{-\frac{M^{3} N}{2}} \prod_{i=1}^{N} \int_{\bigcirc} d \phi_{i} e^{\Delta \tau \sum_{j=1}^{N} \int d^{3} x\left[-\frac{1}{2}\left(\frac{\phi_{j+1}-\phi_{j}}{\Delta \tau}\right)^{2}-\frac{1}{2}\left(\vec{\nabla} \phi_{j}\right)^{2}-\frac{m^{2}}{2} \phi_{j}^{2}-\lambda \phi_{j}^{4}\right]}
$$

Se considerarmos novamente, o limite no qual a variável temporal euclidiana está definida num contínuo, a função de partição assumirá uma forma que é comumente encontrada em livros introdutórios à QFT (PESKIN; SCHROEDER, 1995; MUTA, 1987; RAMOND, 1990).

$$
Z=N^{\prime} \int_{\bigcirc}[d \phi] e^{\int_{0}^{\beta} d \tau \int d^{3} x \mathcal{L}_{E}(\phi)}
$$

Esta constante $N^{\prime}$ será irrelevante no nosso contexto, pois como pode ser observado na expressão da média de um operador em (2.2), ela ocorre tanto no numerador quanto no denominador, o que acarreta em um cancelamento.

Ainda a cerca da expressão (2.25), seu expoente pode ser identificado como a ação no espaço euclidiano $S_{E}$, cuja variável temporal está restrita a um intervalo finito. Ademais, assim como fizemos com a lagrangiana, iremos dividir esta ação em duas partes, $S_{0}$ contendo os termos quadráticos no campo, e $S_{I}$ contendo os termos restantes, que no caso de (2.24) será apenas o potencial $V_{I}=\lambda \phi^{4}$.

$$
Z=N^{\prime} \int_{\bigcirc}[d \phi] e^{S_{0}+S_{I}}
$$

Assumindo que as interações presentes em $S_{I}$ são fracas, torna-se possível expandir $e^{S_{I}}$ em uma série de Taylor, e assim temos

$$
Z=N^{\prime} \int_{\bigcirc}[d \phi] e^{S_{0}} \sum_{l=0}^{\infty} \frac{S_{I}^{l}}{l !} .
$$

Já na sequência, iremos calcular o logaritmo da expressão acima, pois $\ln (Z)$ é recorrente nas expressões de todas as variáveis termodinâmicas definidas em (2.3), e além 
disto, este procedimento irá separar a parte de interação da parte quadrática.

$$
\begin{aligned}
\ln (Z) & =\ln \left[\sum_{l=0}^{\infty} N^{\prime} \int_{\bigcirc}[d \phi] e^{S_{0}} \frac{S_{I}^{l}}{l !}\right] \\
& =\ln \left[N^{\prime} \int_{\bigcirc}[d \phi] e^{S_{0}} \sum_{l=0}^{\infty} \frac{\int_{\bigcirc}[d \phi] e^{S_{0}} \frac{S_{I}^{l}}{l !}}{\int_{\bigcirc}[d \phi] e^{S_{0}}}\right] \\
& =\ln \left[N^{\prime}[d \phi] e^{S_{0}}\right]+\ln \left[1+\sum_{l=1}^{\infty} \frac{1}{l !} \frac{\int_{\bigcirc}[d \phi] e^{S_{0}} S_{I}^{l}}{\int_{\bigcirc}}\right] \\
& =\ln \left(Z_{0}\right)+\ln \left(Z_{I}\right)
\end{aligned}
$$

No termo $\ln \left(Z_{0}\right)$ acima, não existe nenhum efeito das interações produzidas por $V_{I}$, e por isto ele representa a contribuição de um gás ideal. Já o termo $\ln \left(Z_{I}\right)$ contém as médias de todas as potências positivas de $S_{I}$ calculadas no sistema deste gás ideal. Portanto, este procedimento de expansão trata a teoria regida por $S$, como se ela fosse uma teoria regulada por $S_{0}$ com pertubações causadas por $S_{I}$. Para que este método seja convergente, essas pertubações devem se tornar cada vezes menores, ao passo que aumentamos a ordem de aproximação, o que implica na condição $V_{I} \ll 1$.

Agora, com o intuito de analisar o efeito dessa expansão de $\ln (Z)$, estudaremos as ordens iniciais destas perturbações, sendo a primeira delas igual a

$$
\ln \left(Z_{1}\right)=\frac{-\lambda \int_{0}^{\beta} d \tau \int_{0}^{3} x \int_{\circ}[d \phi] e^{S_{0}} \phi^{4}(x, \tau)}{\int_{0}[d \phi] e^{S_{0}}} .
$$

Introduzindo uma expansão em série de Fourier do campo $\phi$ nesta expressão, temos

$$
\begin{aligned}
& \ln \left(Z_{1}\right)=-\lambda \sum_{n_{1}, \ldots, n_{4}} \int_{0}^{\beta} d \tau e^{i\left(\omega_{n_{1}}+\ldots+\omega_{n_{4}}\right) \cdot \tau} \int d^{3} x e^{i\left(\vec{p}_{1}+\ldots+\vec{p}_{4}\right) \cdot \vec{x}} \times \\
& \frac{\prod_{l, q} \int\left[\tilde{\phi}_{l}(q)\right] e^{\tilde{\phi}_{l}(\vec{q})\left[-\frac{1}{2} \beta^{2}\left(\omega_{l}^{2}+\vec{q}^{2}+m^{2}\right)\right] \tilde{\phi}_{-l}(-\vec{q})} \tilde{\phi}_{n_{1}}\left(\vec{p}_{1}\right) \ldots \tilde{\phi}_{n_{4}}\left(\vec{p}_{4}\right)}{\prod_{l, q} \int\left[\tilde{\phi}_{l}(q)\right] e^{\tilde{\phi}_{l}(\vec{q})\left[-\frac{1}{2} \beta^{2}\left(\omega_{l}^{2}+\vec{q}^{2}+m^{2}\right)\right] \tilde{\phi}_{-l}(-\vec{q})}} .
\end{aligned}
$$

Essas integrações em $x$ e $\tau$ são triviais, e culminam em $\beta \delta_{n_{1}+\ldots+n_{4}, 0}$ e $V \delta\left(\vec{p}_{1}+\ldots+\vec{p}_{4}\right)$, respectivamente. Ademais, essas funções delta irão impor a conservação da energia e dos momentos, como já era esperado, pois estamos lidando com campos que representam partículas reais. 
Nos focando agora no numerador de (2.30), não é difícil ver que caso haja no integrando uma potência ímpar de um dos campos $\tilde{\phi}_{n_{i}}\left(\vec{p}_{i}\right)$, esta integral será nula por simetria. Portanto, os únicos termos que irão contribuir em (2.30) serão aqueles contendo potências pares dos campos $\tilde{\phi}$, fato que quando aliado à conservação do quadrimomento resulta nas seguintes condições:

$$
\begin{gathered}
\left(n_{1}, \vec{p}_{1}\right)=-\left(n_{2}, \vec{p}_{2}\right) \text { e }\left(n_{3}, \vec{p}_{3}\right)=-\left(n_{4}, \vec{p}_{4}\right), \text { ou } \\
\left(n_{1}, \vec{p}_{1}\right)=-\left(n_{4}, \vec{p}_{4}\right) \text { e }\left(n_{3}, \vec{p}_{3}\right)=-\left(n_{2}, \vec{p}_{2}\right), \text { ou } \\
\left(n_{1}, \vec{p}_{1}\right)=-\left(n_{3}, \vec{p}_{3}\right) \text { e }\left(n_{2}, \vec{p}_{2}\right)=-\left(n_{4}, \vec{p}_{4}\right) .
\end{gathered}
$$

Além destas condições, devemos também considerar:

$$
(l, \vec{q})=\left(n_{1}, \vec{p}_{1}\right) \text { e }(l, \vec{q})=\left(n_{3}, \vec{p}_{3}\right), \text { combinadas com }(2.31) \text { e }(2.32) ; \text { ou }
$$

$$
(l, \vec{q})=\left(n_{1}, \vec{p}_{1}\right) \text { e }(l, \vec{q})=\left(n_{2}, \vec{p}_{2}\right), \text { combinadas com }(2.33) ;
$$

pois caso contrário, os campos $\tilde{\phi}_{n_{i}}\left(\vec{p}_{i}\right)$ serão fatorados no numerador de $(2.30)$, e a integral restante será idêntica à presente no denominador, resultando em uma constante, que a princípio pode ser ignorada.

Aplicados todos esses condicionamentos, as integrais restantes serão do tipo:

$$
\frac{\int_{-\infty}^{\infty} d x x^{2} e^{-\frac{a x^{2}}{2}}}{\int_{-\infty}^{\infty} d x e^{-\frac{a x^{2}}{2}}}=\frac{-2\left(\frac{d}{d a}\right)\left(\int_{-\infty}^{\infty} d x e^{-\frac{a x^{2}}{2}}\right)}{\int_{-\infty}^{\infty} d x e^{-\frac{a x^{2}}{2}}}=-2 \frac{\frac{d}{d a}\left(\sqrt{\frac{2 \pi}{a}}\right)}{\sqrt{\frac{2 \pi}{a}}}=\frac{1}{a}
$$

Então, poderemos reescrever (2.30), como

$$
\ln \left(Z_{1}\right)=-3 \lambda \beta V\left(T \sum_{n_{1}} \int \frac{d^{3} p_{1}}{2 \pi} \frac{1}{\omega_{n_{1}}^{2}+\vec{p}_{1}^{2}+m^{2}}\right)\left(T \sum_{n_{2}} \int \frac{d^{3} p_{2}}{2 \pi} \frac{1}{\omega_{n_{2}}^{2}+\vec{p}_{2}^{2}+m^{2}}\right) .
$$

Essas duas integrais, tanto a em $p_{1}$, quanto a em $p_{2}$, possuem o mesmo tipo de integrando, $\frac{1}{\omega_{n}^{2}+\vec{p}^{2}+m^{2}}$, que pode ser identificado com o propagador livre, isto é, o propagador de uma teoria regida por $\mathcal{L}_{0}$.

Uma forma simples de verificar tal fato, decorre da substituição de $\mathcal{L}_{0}$ na equação de Euler-Lagrange(PESKIN; SCHROEDER, 1995), esta que é resultado direto do princípio de mínima ação.

$$
\partial_{\mu}\left(\frac{\partial \mathcal{L}}{\partial\left(\partial_{t} \phi\right)}\right)=\frac{\partial \mathcal{L}}{\partial \phi} \stackrel{\left(\mathcal{L}=\mathcal{L}_{0}\right)}{\longrightarrow}\left(-\partial_{\tau}^{2}-\vec{\nabla}^{2}+m^{2}\right) \phi=0
$$


A expressão acima, define a equação de movimento para o campo $\phi$ no ensemble do gás ideal, e a ela podemos associar uma função de Green, que por sua vez pode ser identificada com o propagador livre $D_{0}$, cuja expressão no espaço dos momentos se escreve como

$$
D_{0}\left(\omega_{n}, \vec{p}\right)=\frac{1}{\omega_{n}^{2}+\vec{p}^{2}+m^{2}} .
$$

A presença destes propagadores nos integrandos de (2.37), pode ser interpretada através dos diagramas de Feynman, sendo que estes representam os processos físicos possíveis para cada uma das ordens de aproximação. A interpretação e a construção destes diagramas serão descritas a seguir.

Na expressão (2.37) para o $\ln \left(Z_{1}\right)$, obtivemos duas integrais de mesma estrutura, e cada uma delas representa a integral de um propagador no espaço dos momentos. Desta forma, atrelaremos à essas integrais uma linha que irá conectar dois pontos do espaço-tempo euclidiano, e cuja representação gráfica é

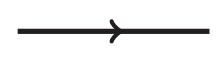

sendo que o sentido desta seta reflete a direção do fluxo do quadrimomento $p_{E}=\left(\omega_{n}, \vec{p}\right)$.

Como $\ln \left(Z_{1}\right)$ resulta da média $\left\langle V_{1}\right\rangle=-\lambda\left\langle\phi^{4}(x, \tau)\right\rangle$, cada uma dessas potências de $\phi$ irá representar um ponto de entrada ou de saída pertencente à um vértice localizado em $(x, \tau)$, conforme o diagrama abaixo.

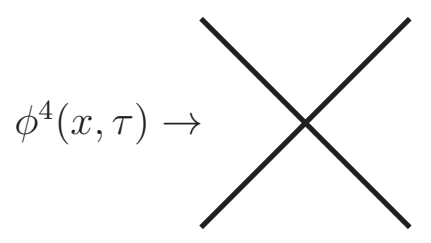

Reescrevendo este diagrama no espaço dos momentos, obtemos

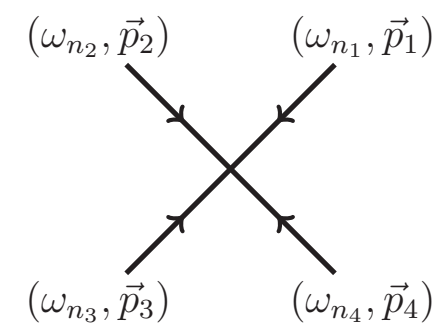

Essas setas, da mesma forma como foi definido para os propagadores, representam a direção do fluxo de $p_{E}$. Ademais, como existem duas possibilidades para essas direções, isto é, ou os momentos estão ingressando no vértice ou esses momentos estão saindo dele, utilizaremos por convenção a primeira dessas possibilidades.

Além do mais, se aplicarmos as condições representadas por (2.31-2.35) em um vértice qualquer, deveremos obrigatoriamente associar cada uma das entradas/saídas de 
(2.42) em pares. Tal conexão deverá contemplar todas as possíveis combinações desses pares, e será feita através dos propagadores, o que resulta no seguinte diagrama para $\ln \left(Z_{1}\right)$,

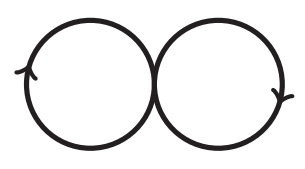

Adicionalmente, mesmo ocorrendo três combinações distintas destes diagramas, todos eles resultam em um mesmo valor numérico, e por isto, teremos

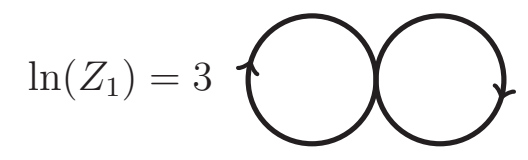

Ao comparar (2.37) com o diagrama acima, vemos que associados à um vértice, temos uma potência de $-\lambda$ proveniente da expansão de $e^{S_{I}}$, e um fator $\beta(2 \pi)^{3} \delta_{\omega_{\text {in }}, \omega_{\text {out }}} \delta\left(\vec{p}_{\text {in }}-\right.$ $\left.\vec{p}_{\text {out }}\right)$ resultante da transformada de Fourier, em que os índices "in" e "out" representam a soma dos quadrimomentos ingressando ou saindo do vértice, respectivamente. Adicionalmente, como as condições de conservação do quadrimomento são obedecidas em (2.37), estas funções delta se resumem ao fator multiplicativo $\beta V$.

Aliás, a comparação feita no parágrafo anterior, também permite concluir que para cada linha teremos um fator

$$
T \sum_{n} \int \frac{d^{3} p}{(2 \pi)^{3}} D_{0}\left(\omega_{n}, \vec{p}\right)
$$

Esses preceitos até agora obtidos a partir de $\ln \left(Z_{1}\right)$, compõem quase que todas as regras de Feynman, todavia é necessário analisar a próxima ordem de aproximação, pois como veremos, ocorrem interferências entre diagramas de ordens superiores. Para tal, vamos inicialmente expandir $\ln \left(Z_{I}\right)$ em relação à $S_{I}$, e após agruparemos os termos com uma mesma potência da constante de acoplamento, conforme

$$
\begin{aligned}
\ln \left(Z_{I}\right) & =\ln \left(1+\sum_{l=1}^{\infty} \frac{\left\langle S_{I}^{l}\right\rangle}{l !}\right)=\sum_{l=1}^{\infty} \frac{\left\langle S_{I}^{l}\right\rangle}{l !}-\frac{1}{2}\left(\sum_{l=1}^{\infty} \frac{\left\langle S_{I}^{l}\right\rangle}{l !}\right)^{2}+\frac{1}{3}\left(\sum_{l=1}^{\infty} \frac{\left\langle S_{I}^{l}\right\rangle}{l !}\right)^{3} \ldots=\left(\left\langle S_{I}^{1}\right\rangle\right)_{\lambda^{1}}+ \\
& +\left(\frac{1}{2}\left\langle S_{I}^{2}\right\rangle-\frac{1}{2}\left(\left\langle S_{I}^{1}\right\rangle\right)^{2}\right)_{\lambda^{2}}+\left(\frac{1}{6}\left\langle S_{I}^{3}\right\rangle-\frac{1}{2}\left\langle S_{I}^{2}\right\rangle\left\langle S_{I}^{1}\right\rangle+\frac{1}{3}\left(\left\langle S_{I}^{1}\right\rangle\right)^{3}\right)_{\lambda^{3}}+\ldots,
\end{aligned}
$$

em que os índices inferiores aos parênteses, indicam qual é a potência de $\lambda$ dos termos internos.

Posto isto, vemos em (2.46), que para $\lambda^{2}$ temos dois termos, sendo que um deles pode ser diretamente calculado com o auxílio de (2.44),

$$
-\frac{1}{2}\left\langle S_{I}^{\prime}\right\rangle^{2}=-\frac{1}{2}\left(\ln \left(Z_{1}\right)\right)^{2}=-\frac{1}{2} \times 3 \bigcirc
$$


Já o outro termo, $\left\langle S_{I}^{2}\right\rangle$, pode ser calculado via integração funcional, como foi feito para $\ln \left(Z_{1}\right)$, ou podemos aproveitar as regras diagramáticas discutidas nos parágrafos anteriores. Escolhendo este último procedimento, iniciaremos com os vértices definidos para $\phi^{4}(x, \tau)$ e $\phi^{4}\left(x^{\prime}, \tau^{\prime}\right)$ :

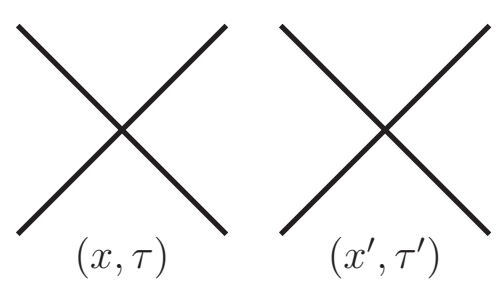

A conexão entre as saídas/entradas destes dois vértices irá gerar três tipos de diagramas, para os quais existem fatores que consideram quantas vezes cada um destes se repete, resultando em
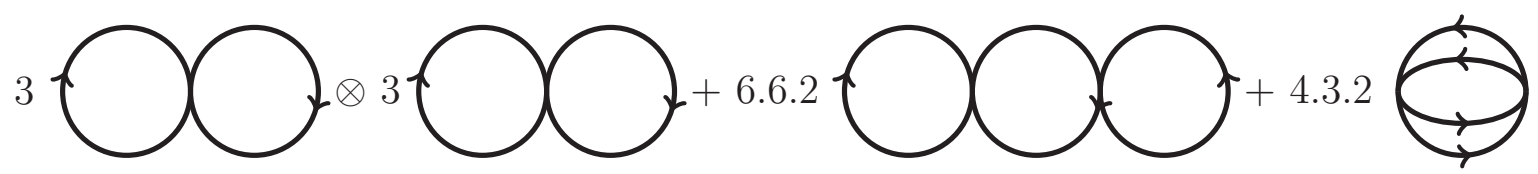

Além destes fatores, ainda existe um fator $\frac{1}{2}$ decorrente da expansão em (2.46). Assim, fica claro que o primeiro diagrama em (2.49) irá cancelar o termo proveniente de $-\frac{1}{2}\left(\ln \left(Z_{1}\right)\right)^{2}$, e portanto, restarão apenas os seguintes diagramas:

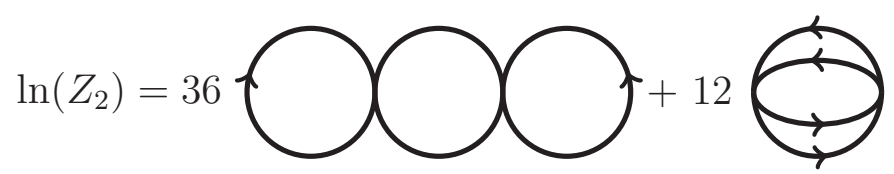

Esses dois diagramas acima, e o contido em (2.44), compartilham uma forma em comum, o que lhes garante a seguinte propriedade: cada uma de suas partes está conectada à todas as outras. Esse procedimento defini os diagramas conexos, e como veremos, eles representam uma classe muito importante de processos físicos.

De forma geral, cancelamentos como o visto em (2.49), ocorrem para todas as ordens da expansão na constante de acoplamento, e não só para $\lambda^{2}$. Para melhor visualizar tal fato, basta retornarmos a expressão (2.46). Nela vemos que para as contribuições proporcionais à $\lambda^{k}$, sempre haverá um termo $\left\langle S_{I}^{k}\right\rangle$ acompanhado de todas as combinações dos termos $\left\langle S_{I}^{a}\right\rangle^{l}$, com a.l $=k$, e mais os produtos $\left\langle S_{l_{1}}^{a_{1}}\right\rangle^{l}\left\langle S_{I}^{a_{2}}\right\rangle^{l_{2}} \ldots$, com $a_{1} \cdot l_{1}+a_{2} . l_{2}+\ldots=k$. Todos esses termos diferentes de $\left\langle S_{I}^{k}\right\rangle$, irão cancelar a parte desconexa do mesmo, restando somente a parte conexa. Demonstrações detalhadas deste fato, que pode ser resumido por

$$
\ln \left(Z_{I}\right)=\sum_{l=0}^{\infty} \frac{1}{l !}\left\langle S_{I}^{l}\right\rangle_{c}
$$

em que $\left\langle S_{I}^{l}\right\rangle_{c}$ representa os diagramas conexos de ordem $l$, são encontradas nos livros (PESKIN; SCHROEDER, 1995) e (KAPUSTA; GALE, 2006). 
Aliás, como os diagramas desconexos são proporcionais à potências do volume maiores do que a unidade, as suas contribuições comprometeriam a extensibilidade de $\ln (Z)$, e consecutivamente das variáveis termodinâmicas como a entropia, a pressão, a energia e a densidade de partículas, e portanto, o seu cancelamento descrito nos parágrafos anteriores já era esperado.

Por fim, agora é possível construir todos os termos que contribuirão com $\ln \left(Z_{I}\right)$, para qualquer ordem de aproximação, através das regras de Feynman, que de forma resumida podem ser escritas como:

1) Construir todos os diagramas de Feynman conexos, e calcular seus respectivos fatores combinatórios;

2) Atribuir um fator $T \sum_{n} \int \frac{d^{3} p}{(2 \pi)^{2}} D_{0}\left(\omega_{n}, \vec{p}\right)$ para cada linha interna destes diagramas;

3) Incluir um fator $-\lambda$ para cada vértice destes diagramas, e considerar a conservação dos quadrimomentos envolvidos neste vértice através da inclusão de fatores do tipo $\beta(2 \pi)^{3} \delta_{\omega_{i} n, \omega_{o} u t} \delta\left(\vec{p}_{i} n-\vec{p}_{o} u t\right)$, ou considerar esta conservação diretamente na composição dos diagramas, como por exemplo,

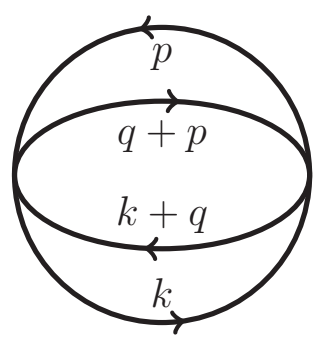

4) Incluir um fator $\beta V$.

\subsection{Amplitudes frontais}

Na seção anterior, apresentamos uma técnica para obter a função de partição a partir de diagramas de Feynman. Todavia, ainda restam por fazer, as integrais nos momentos e a somatória na energia. Na maioria dos casos a ordem na qual efetuamos essas operações, se primeiro as integrais nos momentos ou a somatória nas energias, não irá alterar o resultado, porém se os integrandos não forem analíticos essa invariância não é garantida. E como para a maioria das teorias de calibre, os propagadores e os vértices são analíticos, a menos de alguns limites específicos, não deveremos nos preocupar com essa ordem de integração/soma.

De forma geral, em ordem de um loop, podemos imaginar que a somatória nas frequências de Matsubara se resume a soma de funções $f\left(p_{0}=i \omega_{n}, \vec{p}\right)$ definidas no espaço dos momentos com métrica de Minkowski. Como os valores assumidos pelas frequências $\omega_{n}$, para bósons, são idênticos aos valores dos polos de uma cotangente hiperbólica com 
argumento igual a $\frac{\beta p_{0}}{2}$, existe uma integral no plano complexo de $p_{0}$ que reproduzirá esta soma.

$$
F=\sum_{n} f\left(p_{0}=\omega_{n}, \vec{p}\right)=\frac{1}{2 \pi i} \oint_{C} d p_{0} \frac{1}{2} \operatorname{coth}\left(\frac{\beta p_{0}}{2}\right) f\left(p_{0}, \vec{p}\right)
$$

Essa expressão é resultado direto do cálculo via resíduos, em que consideramos o caminho de integração $C$ conforme representado na figura 1. Equivalentemente, a soma para os férmions pode ser reescrita também em uma integral no plano complexo, bastando somente substituir a cotangente hiperbólica em (2.53) por uma tangente hiperbólica de mesmo argumento.

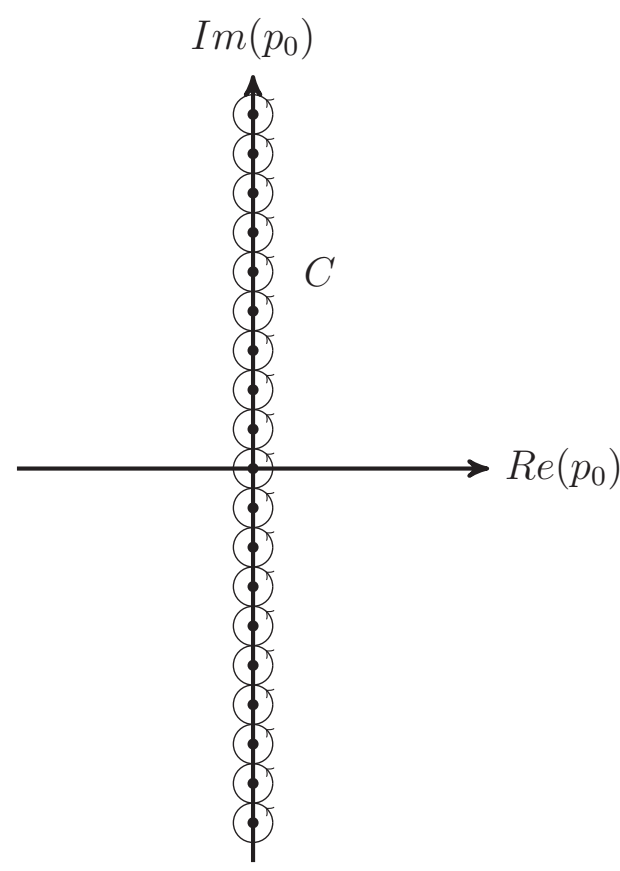

(a)

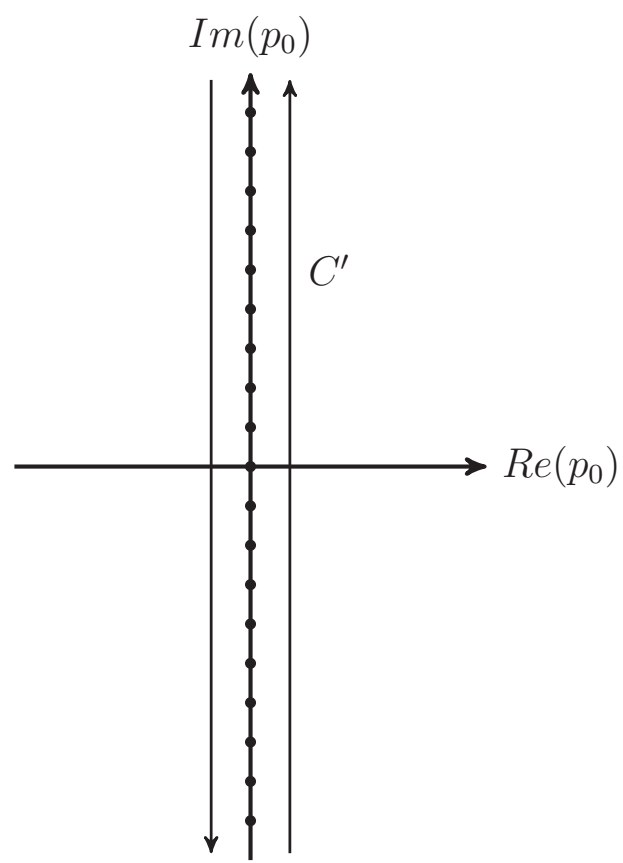

(b)

Figura 1 - (a) Polos de uma cotangente hiperbólica envoltos por caminhos de integração circulares; (b) Caminho de integração formado pela deformação dos caminhos circulares contidos em (a).

Os vários caminhos circulares, componentes do caminho $C$, que envolvem os polos da cotangente hiperbólica, podem ser deformados de maneira a obtermos o caminho $C^{\prime}$ indicado na figura 1. Essa deformação só poderá ser efetuada, se $f\left(p_{0}, \vec{p}\right)$ não possuir nenhum polo no eixo imaginário de $p_{0}{ }^{2}$, caso contrário não poderemos efetuar a integral em (2.53) utilizando o caminho $C^{\prime}$ ao invés do caminho $C$.

2 Essa condição para teorias de calibre depende justamente do calibre que estamos utilizando, por exemplo, para o calibre temporal é necessário adotar um procedimento especial no cálculo das funções de Green, conforme os trabalhos (BRANDT; FRENKEL; MACHADO, 2000; BRANDT; CUADROS-MELGAR; MACHADO, 2003). 


$$
\begin{aligned}
\frac{1}{2 \pi i} \oint_{C^{\prime}} d p_{0} \frac{1}{2} \operatorname{coth}\left(\frac{\beta p_{0}}{2}\right) f\left(p_{0}, \vec{p}\right)=\frac{1}{2 \pi i} \int_{i \infty-\epsilon}^{-i \infty-\epsilon} d p_{0} \frac{1}{2} \operatorname{coth}\left(\frac{\beta p_{0}}{2}\right) f\left(p_{0}, \vec{p}\right)+ \\
+\frac{1}{2 \pi i} \int_{-i \infty+\epsilon}^{i \infty+\epsilon} d p_{0} \frac{1}{2} \operatorname{coth}\left(\frac{\beta p_{0}}{2}\right) f\left(p_{0}, \vec{p}\right)
\end{aligned}
$$

No lado direito da expressão acima, substituiremos $p_{0}$ por $-p_{0}$ na primeira integral, e neste mesmo termo, efetuaremos também a transformação $\vec{p} \rightarrow-\vec{p}$, que não altera o valor de $f\left(p_{0}, \vec{p}\right)$, pois esta função possui uma integração em todas as direções espaciais do quadrimomento $p$. Além disto, levando em conta que a cotangente hiperbólica é uma função impar, teremos

$$
\frac{1}{2 \pi i} \oint_{C} d p_{0} \frac{1}{2} \operatorname{coth}\left(\frac{\beta p_{0}}{2}\right) f\left(p_{0}, \vec{p}\right)=\frac{1}{2 \pi i} \int_{-i \infty+\epsilon}^{i \infty+\epsilon} d p_{0} \frac{1}{2} \operatorname{coth}\left(\frac{\beta p_{0}}{2}\right)[f(p)+f(-p)] .
$$

Para concluir, podemos somar à expressão acima um outro termo com mesmo integrando, porém substituindo o caminho de integração por $C_{\supset}$, que é o semi-círculo infinito com parte real positiva. Isto nos permitirá calcular a integral resultante, definida no caminho fechado $C_{D}$, via teorema dos resíduos, mas desta vez os polos a serem utilizados serão os pertencentes à $f( \pm p)$, e que possuem parte real positiva.

$$
\begin{aligned}
\frac{1}{2 \pi i}\left(\int_{-i \infty+\epsilon}^{i \infty+\epsilon}+\int_{C_{\supset}}\right) d p_{0} \frac{1}{2} \operatorname{coth}\left(\frac{\beta p_{0}}{2}\right)[f(p)+f(-p)]= \\
\frac{1}{2 \pi i} \oint_{C_{D}} d p_{0} \frac{1}{2} \operatorname{coth}\left(\frac{\beta p_{0}}{2}\right)[f(p)+f(-p)]
\end{aligned}
$$

Para que essa expressão acima seja igual a (2.53), a integral definida no caminho $C_{\supset}$ deverá ser nula. Felizmente, isto ocorrerá nos casos das teorias que trataremos neste trabalho, pois elas possuem propagadores que descressem com $p_{0}$, e mesmo quando os vértices dependem da energia, a razão entre eles sempre tenderá a 0 quando $p_{0} \rightarrow \infty$. Aliás, ainda que isso não ocorra, como é o caso da gravitação, as distribuições de Bose-Einstein ou Fermi-Dirac descressem suficientemente rápido para anular as expressões na região com $p_{0}$ grande.

Adicionalmente, $\operatorname{como} \tanh (x)=\operatorname{coth}^{-1}(x)$, ao generalizarmos (2.56) para bósons 
e férminos, efetuaremos as seguintes manipulações:

$$
\begin{aligned}
\frac{1}{2 \pi i} \oint_{C_{D}} d p_{0} \operatorname{coth}^{ \pm 1} & \left(\frac{\beta p_{0}}{2}\right) \frac{[f(p)+f(-p)]}{2}= \\
& =\frac{1}{2 \pi i} \oint_{C_{D}} d p_{0}\left(\frac{e^{\frac{\beta p_{0}}{2}}+e^{-\frac{\beta p_{0}}{2}}}{e^{\frac{\beta p_{0}}{2}}-e^{-\frac{\beta p_{0}}{2}}}\right)^{ \pm 1} \frac{[f(p)+f(-p)]}{2} \\
& =\frac{1}{2 \pi i} \oint_{C_{D}} d p_{0} \frac{[f(p)+f(-p)]}{2} \pm \frac{1}{2 \pi i} \oint_{C_{D}} d p_{0} \frac{1}{e^{\beta p_{0}} \mp 1}[f(p)+f(-p)] .
\end{aligned}
$$

Nesta expressão, vemos que a soma das frequências de Matsubara transformou-se em duas integrais, a primeira idêntica àquela que teríamos se tivéssemos considerando a teoria sem os efeitos da temperatura, adicionada de uma rotação de Wick, isto é, a contribuição do vácuo em um espaço euclidiano; e a segunda integral possuindo a mesma estrutura que teríamos para a QFT usual, mas ponderada por uma distribuição estatística, que para bósons é a distribuição de Bose-Einstein, $N_{B}\left(p_{0}\right)=\frac{1}{e^{\beta p_{0}}-1}$, e para os férmions é a distribuição de Fermi-Dirac, $N_{F}=\frac{1}{e^{\beta p_{0}}+1}$.

Para lidar com esta contribuição do vácuo em (2.57), basta utilizarmos os procedimentos de renormalização desenvolvidos para a QFT usual. Todavia, nosso foco se dará ao outro termo, pois este contém todos os efeitos da temperatura, e é o único que contribui nas equações de transporte, como veremos nos capítulos seguintes.

Antes de prosseguirmos, é importante ressaltar duas características da parte térmica de (2.57), a primeira delas é que as distribuições $N_{B, F}$ evitam o aparecimento de divergências ultravioletas, já que seu decaimento exponencial é suficiente para amortizar qualquer crescimento advindo de $f( \pm p)$. E a segunda, refere-se ao fato de que quando aplicado o limite $T \rightarrow 0$, esta parte térmica se anula, restando somente a contribuição do vácuo.

\subsubsection{Amplitudes frontais para a teoria $\phi^{3}$}

A fim de ilustrar uma das formas de efetuar as integrações em $p_{0}$, contidas na expressão (2.57), vamos utilizar a teoria $\phi^{3}$ com massa nula, que tem sua forma gráfica muito semelhante à QED. As regras de Feynman para a teoria $\phi^{3}$ são muito simples: para cada vértice temos $-\lambda$; e para cada propagador temos $\frac{1}{k^{2}}$, onde $k$ é o quadrimomento do campo $\phi$.

Iniciaremos pelo diagrama de um loop, o tadpole, que é mostrado na figura 2 , e para o qual temos

$$
f^{(1)}(k)=\frac{-\lambda}{(k+p)^{2}}=\frac{-\lambda}{k^{2}}
$$




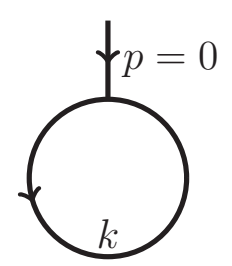

Figura 2 - Ordem mais baixa dos diagramas de um loop com pernas externas, que devido ao seu formato é chamado de tadpole.

A substituição deste $f(k)$ na parte térmica de (2.57), que denotaremos por $S(\beta)$, resulta em

$$
\begin{aligned}
S^{(1)}(\beta) & =\frac{1}{2 \pi i} \oint_{C_{D}} d k_{0} \frac{1}{e^{\beta k_{0}}-1}\left[f^{(1)}(k)+f^{(1)}(-k)\right] \\
& =\frac{1}{2 \pi i} \oint_{C_{D}} d k_{0} \frac{1}{e^{\beta k_{0}}-1}\left[\frac{-\lambda}{k^{2}}+\frac{-\lambda}{(-k)^{2}}\right] \\
& =\frac{-\lambda}{2 \pi i} \oint_{C_{D}} d k_{0} \frac{1}{e^{\beta k_{0}}-1} \frac{2}{\left(k_{0}-|\vec{k}|\right)\left(k_{0}+|\vec{k}|\right)} .
\end{aligned}
$$

O único polo contido na expressão acima, que possui parte real positiva, ocorre quando $k_{0}=|\vec{k}|$. Assim, utilizando o teorema dos resíduos para resolver (2.59), obtemos

$$
S^{(1)}=\frac{\lambda}{|\vec{k}|} \frac{1}{e^{\beta|\vec{k}|}-1} .
$$

O resultado acima pode ser entendido de forma gráfica através de

$$
\begin{aligned}
& \downarrow p=0 \\
& k
\end{aligned}=-\int \frac{d^{3} k}{(2 \pi)^{3}} \frac{1}{|\vec{k}|} \frac{1}{e^{\beta|\vec{k}|}-1}\left\{\begin{array}{r|c}
\vec{k} & p=0 \\
\hline k & k
\end{array}\right\}_{k^{2}=0} .
$$

Aqui vemos a decomposição do tadpole em outro diagrama, que é representado por um propagador interagindo diretamente com um campo externo ao loop. Este tipo de estrutura representa o caso mais simples das amplitudes frontais, que em geral são compostas por estes diagramas do tipo árvore, e como veremos, ocorrerão sempre que efetuarmos uma soma de Matsubara. Para o caso de (2.60) ainda é possível efetuar as integrações restantes na parte espacial dos momentos de forma fechada, porém para as próximas ordens isto não será possível, mesmo no caso de uma teoria escalar.

O próximo diagrama de um loop a ser tratado, será aquele com duas pernas externas, conforme figura 3. Para ele, temos

$$
f^{(2)}(k)=\frac{\lambda^{2}}{k^{2}(k+p)^{2}} .
$$




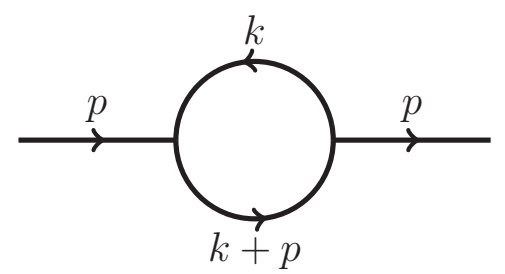

Figura 3 - Diagrama de um loop com duas pernas externas para uma teoria $\phi^{3}$.

Substituindo novamente esta expressão para $f(k)$ na parte térmica de $(2.57)$, culminamos com

$$
\begin{aligned}
S^{(2)}(\beta) & =\frac{\lambda^{2}}{2 \pi i} \oint_{C_{D}} d k_{0} \frac{1}{e^{\beta k_{0}}-1}\left[\frac{1}{k^{2}(k+p)^{2}}+k \rightarrow-k\right]= \\
& =-\left.\lambda^{2} N_{B}\left(k_{0}\right) \frac{1}{2|\vec{k}|} \frac{1}{(k+p)^{2}}\right|_{k_{0}=|\vec{k}|}-\left.\lambda^{2} N_{B}\left(k_{0}\right) \frac{1}{2|\vec{k}+\vec{p}|} \frac{1}{k^{2}}\right|_{k_{0}=|\vec{k}+\vec{p}|-p_{0}}+k \rightarrow-k
\end{aligned}
$$

Além disso, como as pernas externas estão associadas à uma partícula real, com quadrimomento $p$, podemos considerar que suas energias são discretas, $p_{0}=2 \pi l i T$, em que $l$ é um número inteiro. Substituindo esse valor de $p_{0}$ nas distribuições de Bose-Einstein contidas na expressão acima, deduzimos que

$$
\frac{1}{e^{\beta\left(|\vec{k} \mp \vec{p}| \pm p_{0}\right)}-1}=\frac{1}{e^{\beta(|\vec{k} \mp \vec{p}|) \pm \pi l i}-1}=\frac{1}{e^{\beta(|\vec{k} \mp \vec{p}|)}-1},
$$

sendo que essa simplificação também ocorre quando estamos lidando com distribuições de Fermi-Dirac.

Ademais, se aplicarmos o deslocamento $\vec{k} \rightarrow \vec{k}-\vec{p}$ no segundo de (2.63), esta expressão se abreviará em

$$
S^{(2)}(\beta)=-\frac{\lambda^{2}}{|\vec{k}|} \frac{1}{e^{\beta|\vec{k}|}-1}\left[\frac{1}{(k+p)^{2}}+\frac{1}{(k-p)^{2}}\right]_{k^{2}=0} .
$$

Assim como interpretamos $S^{(1)}(\beta)$ de forma gráfica, faremos o mesmo para $S^{(2)}(\beta)$,

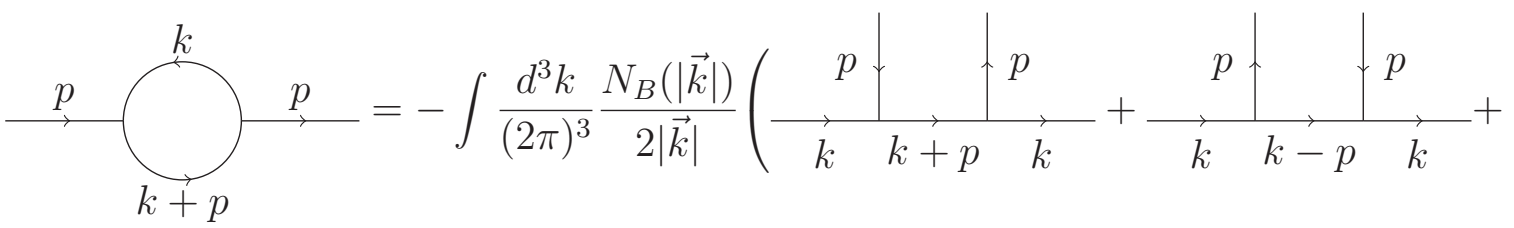

$$
\begin{aligned}
& +k \rightarrow-k)_{k^{2}=0},
\end{aligned}
$$

em que as amplitudes frontais obtidas são permutações cíclicas umas das outras, e contemplam todas as possibilidades destas permutações. Baseando-se nisto e em (2.61), podemos concluir, que as somas nas energias equivalem a cortes nas linhas internas aos loops, respeitados os fluxos e a conservação dos momentos em cada vértice; e assim ao efetuarmos todos esses cortes, obteremos todas as permutações cíclicas desses diagramas. 


\subsubsection{Amplitudes frontais para uma teoria genérica}

As regras para obtenção das amplitudes frontais a partir de diagramas de um loop, observadas na seção anterior, não se restringem apenas à teoria $\phi^{3}$, elas valem também para uma infinidade de outras teorias, e nesta seção estas regras serão generalizadas.

Para considerar as teorias nas quais podemos ter estruturas complexas nos vértices, como é o caso, por exemplo, da QCD, onde alguns de seus vértices dependem dos momentos, definiremos uma função $N$ que contém todas essas estruturas. Além delas, podem ocorrer também nos numeradores, fatores provenientes dos propagadores, especialmente quando desejamos que os denominadores dependam apenas de quantidades escalares, como ocorre, por exemplo, com propagadores fermiônicos,

$$
\frac{1}{\not k-m}=\frac{(\not k+m)}{(\not k-m)(\not k+m)}=\frac{(\not k+m)}{k^{2}-m^{2}} .
$$

Essa dependência do numerador em relação aos momentos internos e externos ao loop, com $n$ pernas externas, será fatorada da seguinte maneira $N=V_{1}\left(k, k+p_{1}\right) V_{2}(k+$ $\left.p_{1}, k+p_{1}+p_{2}\right) \ldots V_{n}\left(k-p_{n}, k\right)$, em que $k$ é o momento interno ao loop, $p_{i}$ são os momentos externos, $p_{n}=-\left(p_{1}+p_{2}+\ldots+p_{n-1}\right)$, e $V_{i}$ são funções dos vértices, que podem conter termos provenientes dos propagadores conectados ao referido vértice, como por exemplo, o numerador de (2.67).

Embora um diagrama de Feynman possa conter várias interações, cada uma delas é independente das outras, isto é, a presença de uma certa interação no gráfico, não altera o caráter das outras, e por isto escolhemos os vértices dependendo apenas dos momentos envolvidos naquela interação específica. De maneira prática, a função $V_{i}$ irá depender dos momentos, $p_{i n}$, pertencentes ao loop que ingressam no vértice $i$, e daqueles momentos, $p_{\text {out }}$, que saem deste vértice; assim definimos a seguinte notação: $V_{i}\left(p_{\text {in }}, p_{\text {out }}\right)$.

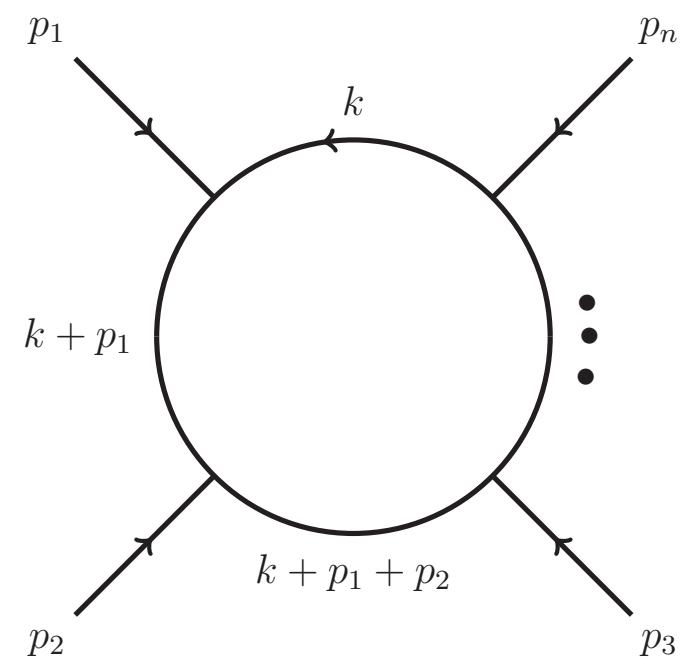

Figura 4 - Diagrama de um loop com $n$ pernas externas para uma teoria genérica. 
Para uma teoria genérica, além das somas/integrações nos momentos, deverão ocorrer também as somas dos outros graus de liberdade, como por exemplo, para a QED, em que é necessário efetuar o traço das matrizes de Dirac provenientes dos propagadores de férmions, ou para a QCD, que além desses traços ainda deveremos somar sob os índices de cor. Todas essas somas serão representadas por $\operatorname{Tr}$ na expressão de $F^{(n)}(p)$, que contém a expressão da amplitude gerada pelo diagrama contido na figura 4, a menos das integrações nos momentos.

$$
F^{(n)}(p)=T \sum_{j} \frac{\operatorname{Tr}\left[V_{1}\left(k, k+p_{1}\right) V_{2}\left(k+p_{1}, k+p_{1}+p_{2}\right) \ldots V_{n}\left(k-p_{n}, k\right)\right]}{k^{2}\left(k+p_{1}\right)^{2} \ldots\left(k-p_{n}\right)^{2}}
$$

Para fins ilustrativos, analisaremos o caso com 3 vértices de interação,

$$
F^{(3)}(p)=T \sum_{j} \frac{\operatorname{Tr}\left[V_{1}\left(k, k+p_{1}\right) V_{2}\left(k+p_{1}, k+p_{1}+p_{2}\right) V_{3}\left(k+p_{1}+p_{2}, k\right)\right]}{k^{2}\left(k+p_{1}\right)^{2}\left(k+p_{1}+p_{2}\right)^{2}} .
$$

Reescrevendo novamente esta soma em uma integral no plano complexo com o auxílio de (2.57), e considerando apenas a parte térmica, obtém-se

$$
\begin{aligned}
S^{(3)}(\beta)= & \frac{1}{2 \pi i} \oint_{C_{D}} d k_{0} N_{B, F}\left(k_{0}\right)\left(\frac{\operatorname{Tr}\left[V_{1}\left(k, k+p_{1}\right) V_{2}\left(k+p_{1}, k+p_{1}+p_{2}\right) V_{3}\left(k+p_{1}+p_{2}, k\right)\right]}{k^{2}\left(k+p_{1}\right)^{2}\left(k+p_{1}+p_{2}\right)^{2}}\right. \\
& \left.+\frac{\operatorname{Tr}\left[V_{1}\left(-k,-k+p_{1}\right) V_{2}\left(-k+p_{1},-k+p_{1}+p_{2}\right) V_{3}\left(-k+p_{1}+p_{2}, k\right)\right]}{k^{2}\left(k-p_{1}\right)^{2}\left(k-p_{1}-p_{2}\right)^{2}}\right) .
\end{aligned}
$$

Em seguida, solucionaremos a integral acima via teorema dos resíduos, o que resultará em

$$
\begin{aligned}
& S^{(3)}(\beta)=-\frac{N_{B, F}(|\vec{k}|)}{2|\vec{k}|}\left(\frac{\operatorname{Tr}\left[V_{1}\left(k, k+p_{1}\right) V_{2}\left(k+p_{1}, k+p_{1}+p_{2}\right) V_{3}\left(k+p_{1}+p_{2}, k\right)\right]}{\left(k+p_{1}\right)^{2}\left(k+p_{1}+p_{2}\right)^{2}}\right)_{k^{2}=0} \\
& -\frac{N_{B, F}\left(\left|\vec{k}+\vec{p}_{1}\right|\right)}{2\left|\vec{k}+\vec{p}_{1}\right|}\left(\frac{\operatorname{Tr}\left[V_{1}\left(k, k+p_{1}\right) V_{2}\left(k+p_{1}, k+p_{1}+p_{2}\right) V_{3}\left(k+p_{1}+p_{2}, k\right)\right]}{k^{2}\left(k+p_{1}+p_{2}\right)^{2}}\right)_{\left(k+p_{1}\right)^{2}=0} \\
& -\frac{N_{B, F}\left(\left|\vec{k}+\vec{p}_{1}+\vec{p}_{2}\right|\right)}{2\left|\vec{k}+\vec{p}_{1}+\vec{p}_{2}\right|}\left(\frac{\operatorname{Tr}\left[V_{1}\left(k, k+p_{1}\right) V_{2}\left(k+p_{1}, k+p_{1}+p_{2}\right)\right]}{k^{2}\left(k+p_{1}+p_{2}\right)^{2}} \times\right. \\
& \left.V_{3}\left(k+p_{1}+p_{2}, k\right)\right)_{\left(k+p_{1}+p_{2}\right)^{2}=0}
\end{aligned}
$$

Agora, efetuaremos no segundo termo acima a transformação $\vec{k} \rightarrow \vec{k}-\vec{p}_{1}$, que não irá alterar os limites de integração da parte espacial de $k$, cuja presença não é explícita, 
mas deve ser considerada. Todavia, esse deslocamento produz $k_{0}=|\vec{k}|-p_{01}$, e assim temos, consecutivamente, $k \rightarrow k-p_{1}$. De forma análoga, faremos $k \rightarrow k-p_{1}-p_{2}$ no terceiro termo acima.

$$
\begin{aligned}
& S^{(3)}(\beta)=-\frac{N_{B, F}(|\vec{k}|)}{2|\vec{k}|}\left(\frac{\operatorname{Tr}\left[V_{1}\left(k, k+p_{1}\right) V_{2}\left(k+p_{1}, k+p_{1}+p_{2}\right) V_{3}\left(k+p_{1}+p_{2}, k\right)\right]}{\left(k+p_{1}\right)^{2}\left(k+p_{1}+p_{2}\right)^{2}}\right. \\
& +\frac{\operatorname{Tr}\left[V_{1}\left(k-p_{1}, k\right) V_{2}\left(k, k+p_{2}\right) V_{3}\left(k+p_{2}, k-p_{1}\right)\right]}{\left(k-p_{1}\right)^{2}\left(k+p_{2}\right)^{2}} \\
& \left.+\frac{\operatorname{Tr}\left[V_{1}\left(k-p_{1}-p_{2}, k-p_{2}\right) V_{2}\left(k-p_{2}, k\right) V_{3}\left(k, k-p_{2}-p_{2}\right)\right]}{\left(k-p_{1}-p_{2}\right)^{2}\left(k-p_{2}\right)^{2}}+(k \rightarrow-k)\right)_{k^{2}=0}
\end{aligned}
$$

Sendo assim, ao identificarmos $p_{3}=-p_{1}-p_{2}$, é notável que o segundo termo entre parenteses equivale ao primeiro termo, se efetuadas as seguintes trocas: $\left(V_{3}, p_{3}\right) \rightarrow\left(V_{1}, p_{1}\right)$ e $\left(V_{1}, p_{1}\right) \rightarrow\left(V_{2}, p_{2}\right)$. Essas trocas configuram uma permutação cíclica das pernas externas, e o mesmo ocorre ao compararmos os outros termos. Adicionalmente, quando esses três termos explicitados em (2.72), forem adicionados aos outros termos com $k \rightarrow-k$, eles irão formar o conjunto de todas as permutações cíclicas das amplitudes frontais provenientes de um diagrama com um loop e 3 pernas externas.

Esse resultado para $n=3$, pode ser facilmente generalizado para um número de vértices qualquer, já que o mecanismo de permutação permanecerá o mesmo, e portanto, a única diferença será a quantidades de termos. Para tanto, vamos demonstrar a primeira permutação cíclica que ocorre no caso mais geral.

$$
\begin{aligned}
& S^{(n)}(\beta)=\left(\frac{-1}{e^{\beta k_{0}} \mp 1} \frac{1}{2 k_{0}} \frac{\operatorname{Tr}\left[V_{1}\left(k, k+p_{1}\right) V_{2}\left(k+p_{1}, k+p_{1}+p_{2}\right) \ldots V_{n}\left(k-p_{n}, k\right)\right]}{k^{2}\left(k+p_{1}\right)^{2} \ldots\left(k-p_{n}\right)^{2}}\right)_{k_{0}=|\vec{k}|} \\
& +\left(\frac{-1}{e^{\beta k_{0}} \mp 1} \frac{1}{2 k_{0}} \frac{\operatorname{Tr}\left[V_{1}\left(k, k+p_{1}\right) V_{2}\left(k+p-{ }_{1}, k+p_{1}+p_{2}\right) \ldots V_{n}\left(k-p_{n}, k\right)\right]}{k^{2}\left(k+p_{1}\right)^{2} \ldots\left(k-p_{n}\right)^{2}}\right)_{k_{0}=\left|\vec{k}+\vec{p}_{1}\right|-p_{01}} \\
& +\ldots+(k \rightarrow-k)
\end{aligned}
$$

A parte térmica da solução de (2.68) via teorema dos resíduos está representada acima. Neste resultado, aplicaremos no segundo termo a transformação $k \rightarrow k-p_{1}$, no terceiro termo a transformação $k \rightarrow k-p_{1}-p_{2}$, e a assim sucessivamente em todos os 
termos restantes.

$$
\begin{aligned}
& S^{(n)}(\beta)=\frac{-N_{B, F}(|\vec{k}|)}{2|\vec{k}|}\left(\frac{\operatorname{Tr}\left[V_{1}\left(k, k+p_{1}\right) V_{2}\left(k+p_{1}, k+p_{1}+p_{2}\right) \ldots V_{n}\left(k-p_{n}, k\right)\right]}{\left(k+p_{1}\right)^{2}\left(k+p_{1}+p_{2}\right)^{2} \ldots\left(k-p_{n}\right)^{2}}\right. \\
& \left.+\frac{\operatorname{Tr}\left[V_{1}\left(k-p_{1}, k\right) V_{2}\left(k, k+p_{2}\right) \ldots V_{n}\left(k-p_{n}-p_{1}, k-p_{1}\right)\right]}{\left(k+p_{2}\right)^{2} \ldots\left(k-p_{n}-p_{1}\right)^{2}\left(k-p_{1}\right)^{2}}+\ldots+(k \rightarrow-k)\right)_{k^{2}=0}
\end{aligned}
$$

Comparando esse primeiro termo, representado na figura 5a, com o segundo, ilustrado na figura $5 \mathrm{~b}$, notam-se as seguintes trocas: $\left(V_{n}, p_{n}\right) \rightarrow\left(V_{1}, p_{1}\right),\left(V_{1}, p_{1}\right) \rightarrow\left(V_{2}, p_{2}\right)$, $\ldots,\left(V_{i}, p_{i}\right) \rightarrow\left(V_{i+1}, p_{i+1}\right), \ldots$ e $\left(V_{n-2}, p_{n-2}\right) \rightarrow\left(V_{n-1}, p_{n-1}\right)$. Portanto, essas transformações representam uma permutação cíclica, e assim os outros termos irão formar todas as permutações cíclicas restantes.

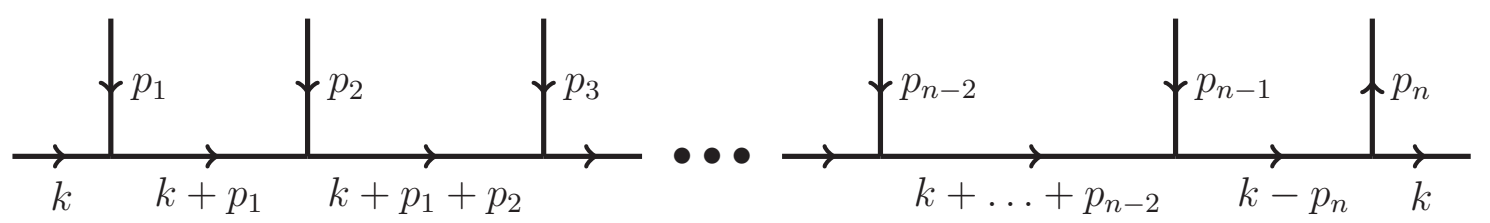

(a)

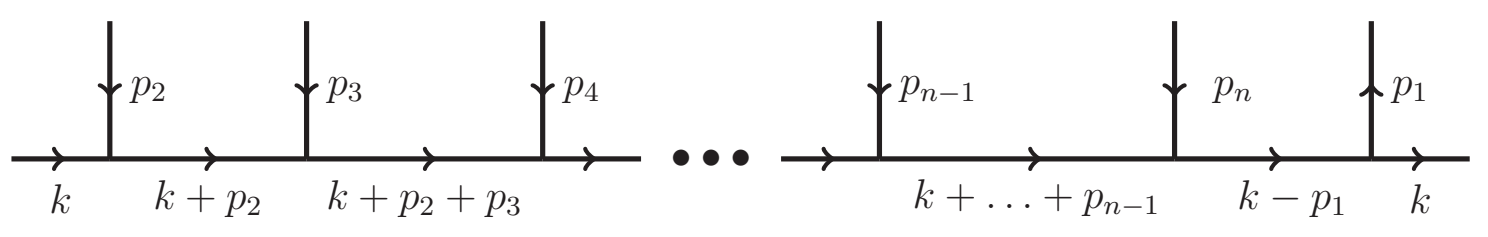

(b)

Figura 5 - (a) Amplitude frontal obtida a partir de um diagrama com um loop e $n$ pernas externas; (b) permutação cíclica de (a).

É interessante notar em (2.74), que todos os campos envolvidos no loop estão na camada de massa nula, isto é, $k^{2}=0$. Isto ocorre, porque todos esses campos representam partículas reais, consecutivamente, devem estar na camada de massa. Caso não estivéssemos utilizando por simplicidade propagadores com massa nula, teriamos $k^{2}=m^{2}$, ou alternativamente, $\omega_{k}=\sqrt{\vec{k}^{2}+m^{2}}$, em que $\omega_{k}$ representa a energia da partícula.

Levando em conta a generalidade do resultado obtido, podemos agora fazer uma continuação analítica das energias externas $p_{0 i}$ para valores contínuos. Embora a natureza discreta de $p_{0 i}$ tenha sido essencial na relação (2.64), nada impede que a continuação analítica seja feita após a integração em $k_{0}$. Com essa prescrição, podemos retornar ao espaço de Minkwoski, e a dependência temporal dos campos externos pode ser estabelecida. Deste modo, é possível descrever o comportamento de sistemas térmicos sob a ação de um campo externo não estático. Por exemplo, podemos investigar a propagação de 
perturbações no meio térmico. De fato, a expressão em termos de amplitudes frontais, aqui derivada, descreve partículas com distribuição térmica interagindo com um campo externo. 



\section{Teorias de calibre}

O conceito de invariância por transformações de calibre surgiu inicialmente nos trabalhos de Maxwell, onde era evidente a invariância tanto do potencial vetor $\vec{A}$, quanto do potencial elétrico $\varphi$, via transformações do tipo $A^{\mu}=(\varphi, \vec{A}) \rightarrow A^{\mu}+\partial^{\mu} \theta$, em que $\theta$ é uma função arbitrária das coordenadas espaciais.

E então, após mais de um século de pesquisa, essas transformações foram generalizadas para estruturas mais complexas no escopo da QFT(YANG; MILLS, 1954; UTIYAMA, 1956), o que resultou na QCD, teoria Eletro-fraca e QED, que explicam, respectivamente, as interações fortes, fracas e eletromagnéticas.

Para melhor exemplificar, o que é a invariância via transformações de calibre, imaginemos uma função de onda associada a um sistema quântico. Ao submetemos esta função de onda a uma transformação do tipo

$$
\psi(x) \rightarrow \psi^{\prime}(x)=e^{i \theta} \psi(x)
$$

$\operatorname{com} \theta$ constante, esta função continuará descrevendo o mesmo sistema, pois as quantidades físicas em mecânica quântica dependem apenas do módulo de $\psi$.

No exemplo do parágrafo anterior, o parâmetro $\theta$ era constante, porém em um caso geral, podemos considerar que ele assume um valor diferente para cada posição do espaço-tempo, o que pode vir a causar uma grande restrição no sistema físico, conforme veremos na seção seguinte.

Adicionalmente, para distinguir entre os casos nos quais ocorre ou não a dependência de $\theta$ com as posições, utilizaremos duas nomenclaturas distintas. No caso de $\theta$ constante, dizemos que o sistema é invariante por uma transformação global, já no outro caso, dizemos que a transformação é local.

Como veremos adiante, as invariâncias via transformações locais nos forçarão a fixar condições de calibre, e como elas são arbitrárias, acredita-se que as quantidades físicas não devem depender dessas escolhas, isto é, a física é independente do calibre utilizado.

Além disso, quando considerada uma transformação local, será necessário o auxílio de um ente físico para tramitar essa informação/condição no espaço-tempo, o que gera a necessidade de incluirmos campos de calibre neste sistema. De um ponto de vista prático, isto ocorre por mera consequência da utilização de uma descrição teórica, que baseia-se no princípio da invariância por transformações de calibre locais.

Embora as transformações globais e locais se assemelhem, suas consequências são completamente distintas. Enquanto, que as transformações globais se prestam a descrever 
degenerescências dos graus de liberdade, as transformações locais representam a ausência de um ou mais graus de liberdade.

\subsection{Transformações de calibre}

Dentre as teorias de calibre existentes, desenvolveremos agora o formalismo para a teoria de Yang-Mills(YANG; MILLS, 1954) em $d+1$ dimensões do espaço-tempo, pois ela representa uma generalização de vários sistemas físicos. Para esta teoria, utilizaremos um grupo de Lie $G$, que por hora será tratado genericamente. Ademais, correlacionada a este grupo, teremos uma álgebra de Lie, a qual associaremos $n$ geradores $T^{a}$, com $a$ assumindo valores inteiros positivos não nulos. Além disso, para esses geradores, utilizaremos uma relação de comutação, (3.2), que introduz as constantes de estrutura fabc desta álgebra.

$$
\left[T^{a}, T^{b}\right]=i f^{a b c} T^{c}
$$

Agora, utilizando a representação fundamental do grupo G, definiremos um campo fermiônico $\psi(x)$, possuindo componentes $\psi_{i}(x), i=1,2, \ldots, N$ e que se transforma, sob a ação de um elemento $U$ do grupo $G$, como

$$
\psi^{\prime}(x)=U(x) \psi(x)=e^{-i T^{a} \theta^{a}(x)} \psi(x) .
$$

Na relação acima, a fim de estudar as simetrias locais associadas a este grupo $G$, fizemos o parâmetro $\theta^{a}$ dependendo das coordenadas espaço-temporais de $x$, e além disso, a lagrangiana $\mathcal{L}_{0}$ de um campo fermiônico livre é trivialmente invariante sob transformações de calibre globais.

$$
\mathcal{L}_{0}=\bar{\psi}(x)\left(i \gamma^{\mu} \partial_{\mu}-m\right) \psi(x)
$$

Adicionalmente, na expressão acima da lagrangiana livre, ou lagrangiana de Dirac, $m$ é a massa associada ao férmion, e $\bar{\psi}=\psi^{\dagger} \gamma^{0}$. Posto isto, se aplicarmos a transformação de calibre (3.3) em (3.4), teremos

$$
\begin{aligned}
\mathcal{L}_{0}^{\prime} & =\bar{\psi}^{\prime}(x)\left(i \gamma^{\mu} \partial_{\mu}-m\right) \psi^{\prime}(x)=\bar{\psi}(x) U^{-1}(x)\left(i \gamma^{\mu} \partial_{\mu}-m\right) U(x) \psi(x) \\
& =\bar{\psi}(x)\left(i \gamma^{\mu} \partial_{\mu}-m\right) \psi(x)+i \bar{\psi}(x) U^{-1}(x) \gamma^{\mu} \partial_{\mu}(U(x)) \psi(x) \\
& =\mathcal{L}_{0}+i \bar{\psi}(x) U^{-1}(x) \gamma^{\mu} \partial_{\mu}(U(x)) \psi(x) .
\end{aligned}
$$

Aqui vemos, que $\mathcal{L}_{0}$ não é invariante via a transformação de calibre definida pelo conjunto dos geradores $T^{a}$. E assim, com o intuito de encontrar uma lagrangiana, que possua essa invariância, iremos utilizar o seguinte ansatz:

$$
D_{\mu}=\partial_{\mu}-i g A_{\mu}=\partial_{\mu}-i g T^{a} A_{\mu}^{a}
$$


em que o fator $-i$ foi introduzido por pura conveniência, e $g$ funcionará como uma constante de acoplamento das interações entre os campos de calibre, $A_{\mu}$, e os campos fermiônicos, conforme veremos. Esse operador $D_{\mu}$, que é referido na literatura como derivada covariante, irá substituir $\partial_{\mu}$ em (3.4), pois é justamente esta derivada quem gera a contribuição a mais contida em (3.3). Para que não ocorra nenhuma confusão, renomearemos esta nova lagrangiana como $\mathcal{L}_{F}$.

Para que $\mathcal{L}_{F}$ seja invariante por transformações de calibre, devemos substituir (3.6) em (3.4), e impor a invariância do termo cinético,

$$
\begin{array}{r}
\left(D_{\mu} \psi(x)\right)^{\prime}=U(x) D_{\mu} \psi(x) \\
{\left[\left(\partial_{\mu}-i g A_{\mu}\right) \psi(x)\right]^{\prime}=U(x)\left(\partial_{\mu}-i g A_{\mu}\right) \psi(x)} \\
A_{\mu}^{\prime} U(x)=U(x) A_{\mu}-\frac{i}{g} \partial_{\mu} U(x) .
\end{array}
$$

Ademais, como U é elemento de um grupo, ele admite inversa, e então

$$
A_{\mu}^{\prime}=U(x) A_{\mu} U^{-1}(x)-\frac{i}{g}\left(\partial_{\mu} U(x)\right) U^{-1}(x) .
$$

Essa expressão define a transformação de calibre para os campos $A_{\mu}$, e para que ela possa ser reescrita em função dos geradores, consideraremos o parâmetro $\theta$ como um infinitesimal, desta forma $U(x) \approx 1-i T^{b} \theta^{b}(x)$, e

$$
\begin{aligned}
& T^{a} A_{\mu}^{\prime}=\left(1-i T^{b} \theta^{b}(x)\right) T^{a} A_{\mu}^{a}\left(1+i T^{b} \theta^{b}(x)\right)-\frac{i}{g}\left[\partial_{\mu}\left(1-i T^{b} \theta^{b}(x)\right)\right]\left(1+i T^{b} \theta^{b}(x)\right) \\
& T^{a} A_{\mu}^{\prime}=T^{a} A_{\mu}^{a}+i\left(T^{a} T^{b}-T^{b} T^{a}\right) A_{\mu}^{a} \theta^{b}(x)-\frac{1}{g} T^{a} \partial_{\mu} \theta^{a}(x)+\mathcal{O}\left(\theta^{2}\right)
\end{aligned}
$$

Todavia, ao desprezarmos os termos com ordem quadrática em $\theta$, substituirmos (3.2) no termo contendo a comutação entre $T^{a}$ e $T^{b}$, e utilizarmos a antissimetria das constantes de estrutura na permutação entre dois de seus índices, culminamos com

$$
A_{\mu}^{\prime a}=A_{\mu}^{a}+f^{a b c} \theta^{b}(x) A_{\mu}^{c}-\frac{1}{g} \partial_{\mu} \theta^{a}(x) .
$$

Assim, todos os termos de $\mathcal{L}_{F}$ estão definidos, e suas relações de transformação estabelecidas. Porém, como $\mathcal{L}_{F}$ já não mais depende somente do campo fermiônico, ela deixará de descrever apenas campos livres, fato explicitado pelo termo de interação sublinhado na expressão abaixo.

$$
\mathcal{L}_{F}=\bar{\psi}(x)\left(i \gamma^{\mu} D_{\mu}-m\right) \psi(x)=\bar{\psi}(x)\left(i \gamma^{\mu} \partial_{\mu}-m\right) \psi(x)+g \bar{\psi}(x) \gamma^{\mu} A_{\mu} \psi(x)
$$

Além disso, o último termo desta expressão é exatamente o termo de interação entre elétrons e fótons na QED, caso identifiquemos $g=-e$, e o grupo de Lie $G$ com 
o grupo unitário unidimensional U(1). Isto ocorre, pois a QED é o caso mais simples desta teoria de Yang-Mills desenvolvida até agora. Inspirando-se nisto, é possível propôr uma generalização da lagrangiana de Maxwell, $\mathcal{L}_{\max }=-\frac{1}{4} F^{\mu \nu} F_{\mu \nu}$. Para tanto, devemos, primeiramente, encontrar um paralelo aos tensores eletromagnéticos, $F^{\mu \nu}$. E como na QED, uma forma de definir esses tensores, é através de

$$
\left[\partial_{\mu}+i q A_{\mu}, \partial_{\mu}+i q A_{\mu}\right]=i q F_{\mu \nu}
$$

em que $q$ é a carga do férmion e $A_{\mu}$ é o potêncial vetor, conclui-se que uma extensão natural seria

$$
\left[D_{\mu}, D_{\nu}\right]=-i g T^{a} F_{\mu \nu}^{a}
$$

O cálculo direto deste comutador, juntamente com o uso da relação (3.2), resulta em

$$
F_{\mu \nu}^{a}=\partial_{\mu} A_{\nu}^{a}-\partial_{\nu} A_{\mu}^{a}+g f^{a b c} A_{\mu}^{b} A_{\nu}^{c}
$$

E assim encontramos o tensor equivalente a $F_{\mu \nu}$, que além de conter os termos usuais da QED, isto é, as derivadas dos campos $A_{\mu, \nu}$, contém também um termo que acopla estes campos, o que altera toda a dinâmica das partículas associadas à $A_{\mu, \nu}$. Agora, em comparação à $\mathcal{L}_{\max }$, definiremos uma lagrangiana contendo os campos de calibre,

$$
\mathcal{L}_{B}=-\frac{1}{4} F^{a \mu \nu} F_{\mu \nu}^{a}
$$

Esta lagrangiana, além de conter termos quadráticos em $A$, que formam a parte cinética de $\mathcal{L}_{B}$, possui também dois tipos de auto-interações destes campos. A primeira delas é proveniente de um termo cubico em $A$, e a segunda é gerada por um termo quártico, cujos diagramas de Feynman constam nas figuras $6 \mathrm{~b}$ e 6c, respectivamente.

Ainda sobre essas auto-interações, elas estão presentes apenas nos casos em que o grupo $G$ é não abeliano, pois elas surgem quando substituímos (3.2) em (3.9) para obter (3.10). Caso contrário, o comutador entre $T^{a}$ e $T^{b}$ em (3.9) seria nulo, e consecutivamente, $\delta A$ não dependeria do próprio $A$, o que acarretaria apenas no termo quadrático da lagrangiana $\mathcal{L}_{B}$.

Adicionalmente, a diferença entre teorias abelianas e não abelianas pode ser exemplificada ao compararmos a QED com a QCD. A primeira, como já foi dito, possui suas simetrias descritas pelo grupo U(1), que é um grupo abeliano, em contrapartida, as simetrias da QCD são descritas pelo grupo SU(3), que além de não abeliano, possui um maior número de graus de liberdade, ou de cargas associadas ao grupo ${ }^{1}$. E por este motivo, a QCD possui um comportamento assimptótico, o que dificulta em muito seus cálculos, especialmente o uso de métodos perturbativos.

1 Segundo o teorema de Noether, é possível associar a cada simetria do sistema, que ocorre via transformações contínuas, uma quantidade conservada, e estas são comumente chamadas de cargas. 


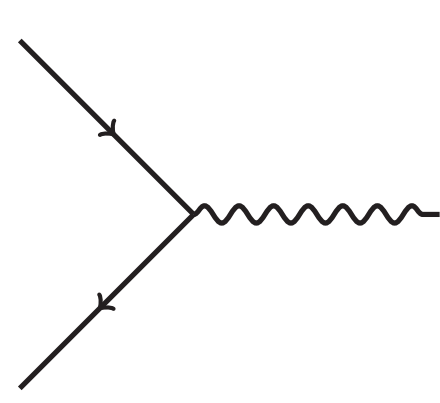

(a)

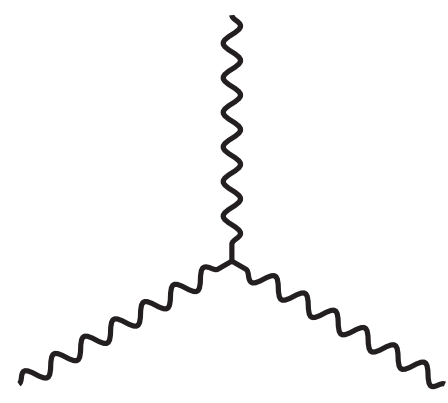

(b)

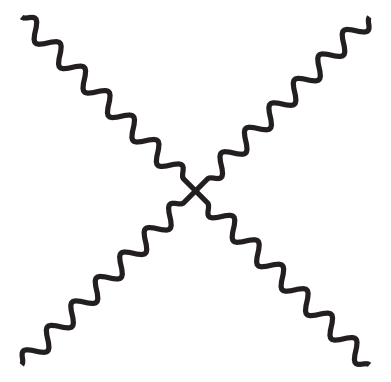

(c)

Figura 6 - (a) Vértice de interação entre férmions e bósons ; (b) Vértice da auto-interação de 3 pontos dos bósons; (c) Vértice da auto-interação de 4 pontos dos bósons.

Agora, pensando em uma teoria mais geral possível, surge a questão: existirá mais algum tipo de interação, além desses três contidos na figura 6? Caso existam, esperamos que esses termos adicionais à lagrangiana de Yang-Mills, $\mathcal{L}_{Y M}$, sejam invariantes por transformações de Lorentz, inversão espacial e reversão temporal. Exemplos de termos com essas propriedades, em mais baixa ordem, são

$$
\begin{aligned}
& \bar{\psi} A^{\mu} A_{\mu} \psi, \quad \bar{\psi} \partial_{\mu} A^{\mu} \partial_{\nu} A^{\nu} \psi, \quad \bar{\psi} \gamma^{\mu} A_{\mu} A^{\nu} A_{\nu} \psi \quad \bar{\psi} \gamma^{\alpha} A_{\alpha} \partial_{\mu} A^{\mu} \partial_{\nu} A^{\nu} \psi \ldots \\
& \gamma^{\mu} A_{\mu} A^{\nu} A_{\nu} A^{\alpha} A_{\alpha}, \quad \partial^{\mu} A_{\mu} A^{\nu} A_{\nu} A^{\alpha} A_{\alpha}, \quad \partial^{\mu} A_{\mu} \partial_{\beta} A_{\alpha} \partial^{\beta} A^{\alpha} A^{\delta} A_{\delta} \ldots
\end{aligned}
$$

Embora todos esses termos possuam as propriedades físicas descritas no parágrafo anterior, eles não são invariantes via transformação de calibre, quando tratados individualmente. Em contrapartida, algumas combinações destes termos, como os escritos abaixo, possuem essa invariância adicional.

$$
\bar{\psi} F^{\mu \nu} F_{\mu \nu} \psi, \quad F^{\mu \alpha} F_{\alpha}^{\beta} F_{\beta \mu}, \ldots
$$

Porém, se caso utilizássemos esses termos na lagrangiana e/ou outros com potências maiores dos campos, surgiriam problemas de renormalização. Uma forma de visualizar esse problema é efetuando uma análise dimensional nas constantes que viriam a multiplicar esses termos. Não é difícil notar que estas constantes teriam dimensões mássicas negativas, o que implica em não renormalizabilidade (MUTA, 1987).

Sendo assim, ao adicionarmos a renormalizabilidade à nossa lista de propriedades desejadas, nos restarão apenas $\mathcal{L}_{F}$ e $\mathcal{L}_{B}$. No entanto, para esta última lagrangiana, ainda não verificamos explicitamente sua invariância por transformações de calibre, posto isto, iniciaremos essa demonstração com o cálculo da transformação do tensor $F$,

$$
F_{\mu \nu}^{\prime a}=\partial_{\mu} A_{\nu}^{\prime a}-\partial_{\nu} A_{\mu}^{\prime a}+g f^{a b c} A_{\mu}^{\prime b} A_{\nu}^{\prime c} .
$$

Como a expressão acima irá se decompor em muitos termos, olharemos cada parte dela isoladamente, sendo a primeira composta pelos termos com as derivadas dos campos 
de calibre,

$$
\begin{aligned}
\partial_{\mu} A_{\nu}^{\prime a}-\partial_{\nu} A_{\mu}^{a} & =\left[\partial_{\mu} A_{\nu}^{a}+f^{a b c}\left(A_{\nu}^{c} \partial_{\mu} \theta^{b}+\theta^{b} \partial_{\mu} A_{\nu}^{c}\right)-\frac{1}{g} \partial_{\mu} \partial_{\nu} \theta^{a}\right] \\
& -\left[\partial_{\nu} A_{\mu}^{a}+f^{a b c}\left(A_{\mu}^{c} \partial_{\nu} \theta^{b}+\theta^{b} \partial_{\nu} A_{\mu}^{c}\right)-\frac{1}{g} \partial_{\nu} \partial_{\mu} \theta^{a}\right] \\
& =\partial_{\mu} A_{\nu}^{a}-\partial_{\nu} A_{\mu}^{a}+f^{a b c}\left(A_{\nu}^{c} \partial_{\mu} \theta^{b}-A_{\mu}^{c} \partial_{\nu} \theta^{b}\right)+f^{a b c} \theta^{b}\left(\partial_{\mu} A_{\nu}^{c}-\partial_{\nu} A_{\mu}^{c}\right) .
\end{aligned}
$$

Já para o termo quadrático de (3.18), vamos analisar diretamente a sua variação,

$$
\begin{aligned}
g f^{a b c}\left(A_{\mu}^{\prime b} A_{\nu}^{\prime c}-A_{\mu}^{b} A_{\nu}^{c}\right) & =\delta g f^{a b c} A_{\mu}^{b} A_{\nu}^{c}=g f^{a b c}\left[\left(\delta A_{\mu}^{b}\right) A_{\nu}^{c}+A_{\mu}^{b}\left(\delta A_{\nu}^{c}\right)\right] \\
& =f^{a b c}\left[g\left(f^{c i j} A_{\mu}^{b} A_{\nu}^{j}+f^{b i j} A_{\nu}^{c} A_{\mu}^{j}\right) \theta^{i}-A_{\mu}^{b} \partial_{\nu} \theta^{c}-A_{\nu}^{c} \partial_{\mu} \theta^{b}\right],
\end{aligned}
$$

que pode ser simplificada se utilizarmos a identidade de Jacobi,

$$
f^{a b c} f^{c i j}+f^{a c j} f^{c i b}+f^{a c i} f^{b j c}=0,
$$

e assim obteremos

$$
\delta\left(g f^{a b c} A_{\mu}^{b} A_{\nu}^{c}\right)=f^{a b c}\left(g f^{c i j} A_{\mu}^{i} A_{\nu}^{j} \theta^{b}-A_{\mu}^{b} \partial_{\nu} \theta^{c}-A_{\nu}^{c} \partial_{\mu} \theta^{b}\right) .
$$

Somando (3.19) à este resultado, concluímos que a variação do tensor $\mathrm{F}$ via transformação de calibre se resume a

$$
\delta F_{\mu \nu}^{a}=f^{a b c} F_{\mu \nu}^{c} \theta^{b}
$$

Consequentemente, a variação de $\mathcal{L}_{B}$ será igual a:

$$
\delta\left(-\frac{1}{4} F^{a \mu \nu} F_{\mu \nu}^{a}\right)=-\frac{1}{2} F^{a \mu \nu} \delta F_{\mu \nu}^{a}=-\frac{1}{2} f^{a b c} F^{a \mu \nu} F_{\mu \nu}^{c} \theta^{b}=0,
$$

em que última passagem segue da antissimetria das constantes de estrutura.

Assim, concluímos que

$$
\mathcal{L}_{Y M}=\mathcal{L}_{F}+\mathcal{L}_{B}=\bar{\psi}(x)\left(i \gamma^{\mu} \partial_{\mu}-m\right) \psi(x)-\frac{1}{4} F^{a \mu \nu} F_{\mu \nu}^{a}
$$

é a forma mais geral de uma lagrangiana renormalizável e invariante por transformações de calibre.

\subsection{Regras de Feynman para as teorias de calibre}

No apêndice A, definimos o funcional gerador $Z[J]$, e um método de como obter os propagadores da teoria através dele. Ao aplicarmos este método na parte fermiônica livre de (3.25), isto é, desconsiderando qualquer termo que não seja estritamente quadrático no campo fermiônico, obteremos trivialmente o propagador dos férmions,

$$
\langle T \bar{\psi}(x) \psi(y)\rangle=\left.\frac{1}{Z[0]}\left[\frac{-i \delta}{\delta J_{\bar{\psi}}(x)} \frac{-i \delta}{\delta J_{\psi}(y)} Z[J]\right]\right|_{J=0}=\int \frac{d^{4} k}{(2 \pi)^{4}} \frac{i}{\not k-m} e^{-i k(x-y)} .
$$


Agora, ao tentarmos fazer o mesmo para os campos de calibre encontramos um problema, pois para que possamos escrever $Z[J]$ na forma

$$
\int[d \phi] e^{i \int d^{4} x \frac{1}{2} \phi \cdot Q \cdot \phi-V(\phi)+J \cdot \phi}=e^{V\left(\frac{\partial}{\partial J}\right)} e^{\frac{1}{2} \phi \cdot Q^{-1} \cdot \phi},
$$

é necessário que o operador quadrático, Q, admita inversa, porém isto não ocorre com

$$
Q^{\mu \nu}=g^{\mu \nu} \partial^{2}-\partial^{\mu} \partial^{\nu}
$$

Essa não invertibilidade de (3.28), é evidenciada quando aplicamos esse operador em um campo de calibre com configuração $A_{\mu}^{\theta}(x)=\partial_{\mu} \theta(x)$, pois

$$
\left(g^{\mu \nu} \partial^{2}-\partial^{\mu} \partial^{\nu}\right) \partial_{\mu} \theta(x)=0
$$

e assim vemos que uma infinidade de estados possuem auto-valor nulo em relação à $Q$, e por isto, este não possui inversa.

Ademais, quando substituímos $A_{\mu}^{\theta}(x)=\partial_{\mu} \theta(x)$ em

$$
Z[0]=\int[d A] e^{i \int d^{4} x \frac{1}{2}\left[A_{\nu}\left(g^{\mu \nu} \partial^{2}-\partial^{\mu} \partial^{\nu}\right) A_{\mu}\right]},
$$

o argumento da exponencial irá se anular, e como $\theta(x)$ é definido para os infinitos pontos do espaço-tempo, haverão infinitas configurações de $A$ a serem integradas, e assim $Z[0]$ irá divergir.

Para entendermos melhor esta divergência de um ponto de vista físico, utilizemos uma teoria modelo, cuja lagrangiana irá diferir de $\mathcal{L}_{B}$ apenas pela adição de um termo mássico, isto é, os bósons estarão na camada de massa não nula.

Antes de prosseguirmos, é importante entender a ação do grupo $S U(N)$, que podemos sumarizar como uma rotação unitária no espaço $N$-dimensional, de modo a manter invariante o produto $v_{1}^{*} \cdot v_{2}$ de dois vetores com $N$ dimensões, em que $v_{1}^{*}$ é o ajunto do vetor $v_{1}$.

Dito isso, a simetria desta teoria modelo quanto as transformações do grupo $S U(N)$ se daria da seguinte forma: inicialmente transladaríamos os campos bosônicos para um referencial inercial próprio, e consecutivamente efetuaríamos as rotações nestes campos.

Quando efetuadas nesta ordem, essas operações mantêm a teoria modelo invariante para qualquer que seja a direção das rotações, porém a primeira dessas transformações não pode ser efetuada para os campos $A$, pois estes não possuem massa, o que nos impede de definir um referencial inercial próprio.

Portanto, a lagrangiana (3.25) apresentará apenas simetria por rotações efetuadas ao redor do eixo definido pela direção do movimento dos campos. Um exemplo disto são os fótons na QED, cujos dois graus de liberdade relativos às rotações são definidos pelas duas polarizações transversais à direção do seu movimento. 


\subsubsection{Método de Faddeev-Popov}

Para lidar com esse problema de inversão do operador (3.28), Faddeev e Popov (FADDEEV; POPOV, 1967) desenvolveram um método, que considera as contribuições para os funcionais geradores de diferentes estados, porém equivalentes, apenas uma única vez, pois estas configurações de campos, embora difiram entre si por transformações de calibre, representam um mesmo sistema físico. Assim, eles conseguiram fatorar as infinitas contribuições de cada uma das transformações de calibre definidas em cada ponto do espaço-tempo.

A fim de ilustrar este método, iniciemos com o funcional gerador

$$
Z_{B}[0]=\int[d A] e^{i \int d^{4} x\left[-\frac{1}{4} F_{\mu \nu}^{a} F a \mu \nu\right]},
$$

que contem apenas $\mathcal{L}_{B}$, pois é ele quem dita a dinâmica dos campos $A$.

Dentre as infinitas possibilidades de transformações de calibre para $A$, escolheremos uma delas ao impor a condição $G(A)=0$, que fixa as configurações de $A$ a serem consideradas em $Z_{B}[0]$. Uma forma de introduzir esta condição em (3.31) é através da seguinte definição da unidade,

$$
1=\int d \theta(x) \delta\left(G\left(A^{\theta}\right)\right) \operatorname{det}\left(\frac{\delta G\left(A^{\theta}\right)}{\delta \theta}\right)
$$

em que $\left(A^{\theta}\right)_{\mu}^{a}=A_{\mu}^{a}+\frac{1}{g} D_{\mu} \theta^{a}$.

A substituição desta transformação $\left(A^{\theta}\right)_{\mu}^{a}$ em (3.31), mantém a forma do funcional gerador, pois como já foi verificado, a ação $S(A)$ é invariante via transformações de calibre. Da mesma forma, a medida de integração também será invariante, posto que a transformação para os campos $A$ é composta de uma translação juntamente com uma rotação unitária, e ambas operações não alteram

$$
[d A]=\prod_{x} \prod_{a, \mu} d A_{\mu}^{a}=\prod_{x} \prod_{a, \mu}\left(d A_{\mu}^{a}\right)^{\theta}=\left[d A^{\theta}\right]
$$

Portanto, inserindo (3.32) em (3.31), e aplicando $A_{\mu}^{a} \rightarrow\left(A^{\theta}\right)_{\mu}^{a}$, temos

$$
\int[d A] e^{i S(A)}=\int\left[d A^{\theta}\right] \int[d \theta] e^{i S\left(A^{\theta}\right)} \delta\left(G\left(A^{\theta}\right)\right) \operatorname{det}\left(\frac{\delta G\left(A^{\theta}\right)}{\delta \theta}\right)
$$

Agora, explorando novamente a invariância deste termo, retornaremos a utilizar o campo $A_{\mu}^{a}$. Além disto, a derivada da condição de calibre é independe de $\theta$, uma vez que $\left(A^{\theta}\right)_{\mu}^{a}$ é linear neste parâmetro, e portanto podemos fatorar a integração em $\theta$,

$$
\int[d A] e^{i S(A)}=\left(\int[d \theta]\right) \int[d A] e^{i S(A)} \delta(G(A)) \operatorname{det}\left(\frac{\delta G\left(A^{\theta}\right)}{\delta \theta}\right) .
$$


Posto isto, vemos que essa integral no parâmetro $\theta$ contribuirá apenas com um fator multiplicativo, infinito, porém constante. E como já foi discutido, constantes multiplicativas nos funcionais geradores não contribuem nos valores de quantidades físicas, desta forma, eliminamos a divergência gerada pelas transformações de calibre.

Para continuar com o procedimento de quantização, devemos explicitar a função $G(A)^{2}$, que escolheremos satisfazer o calibre de Lorenz generalizado:

$$
G(A)=\partial^{\mu} A_{\mu}^{a}(x)-\omega^{a}(x),
$$

e como essa função $\omega(x)$ é arbitrária, iremos somar(integrar) sob todas as suas possibilidades. Adicionalmente, essa integração se dará com o auxílio da função $e^{-i \int d^{4} x \frac{\omega^{2}}{2 \xi}}$, que funcionará como um peso, e em que $\xi$ é uma constante positiva a ser escolhida.

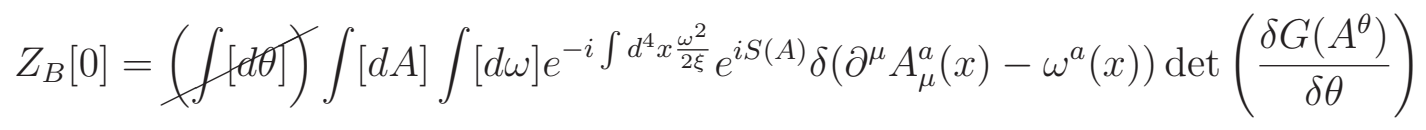

Ao efetuarmos a integral em $\omega$ com o auxílio da função delta, obteremos uma nova lagrangiana para os campos $A$, que possui um termo adicional,

$$
\mathcal{L}_{B}=-\frac{1}{4} F_{\mu \nu}^{a} F^{a \mu \nu}+\frac{1}{2 \xi}\left(\partial^{\mu} A_{\mu}^{a}\right)^{2} .
$$

O termo destacado em (3.38), modifica o termo quadrático de $\mathcal{L}_{B}$, e consecutivamente,

$$
Q^{\mu \nu}=g^{\mu \nu} \partial^{2}+\left(1-\frac{1}{\xi}\right) k^{\mu} k^{\nu}
$$

Desta forma, resultamos com uma expressão para $Q^{\mu \nu}$ que possui inversa bem definida, o que nos permite obter o propagador para os campos de calibre,

$$
\left\langle A_{\mu}^{a}(x) A_{\nu}^{b}(y)\right\rangle=\int \frac{d^{4} k}{(2 \pi)^{4}} \frac{-i}{k^{2}}\left(g_{\mu \nu}-(1-\xi) \frac{k^{\mu} k^{\nu}}{k^{2}}\right) \delta^{a b} e^{-i k(x-y)} .
$$

Ao fixarmos o calibre, foi gerado um fator na expressão acima proporcional à $(1-\xi)^{3}$, e como as quantidades físicas não podem depender de uma constante arbitrária,

2 Existem várias formas de fixarmos as transformações de calibre, dentre as mais utilizadas temos:

Calibre de Lorenz - $G(A)=\partial^{\mu} A_{\mu}$;

Calibre de Coulomb - $G(A)=\partial^{\mu} A_{\mu}$, com $\partial^{\mu}=(0, \vec{\partial})$;

Calibre temporal - $G(A)=t^{\mu} A_{\mu}$, com $t^{\mu}=(1, \overrightarrow{0})$;

Calibre axial - $G(A)=\eta^{\mu} A_{\mu}$, com $\eta$ definindo uma direção fixa no espaço-tempo.

Cada um desses calibres é mais adequado para certas aplicações, por exemplo, o calibre de Lorenz e o axial são invariantes por transformações de Lorentz, enquanto que o calibre temporal e o de Coulomb fixam referenciais especiais.

3 A escolha deste parâmetro $\xi$ será de acordo com cada aplicação, sendo que três das mais utilizadas são: o calibre de Feynman-'t Hooft $\xi=1$, o calibre de Landau $\xi=0$ e o calibre de Yennie $\xi=3$. 
qualquer resultado proveniente deste fator deverá ser nulo. Uma das formas de provar este fato é utilizando as identidades de Ward (WARD, 1950), conforme feito em (PESKIN; SCHROEDER, 1995).

Agora, o único fator que nos resta lidar em (3.37), é o determinante da derivada funcional da condição de calibre pela função parâmetro $\theta$. E como

$$
\frac{\partial}{\partial \theta}(G(A))=\frac{\partial}{\partial \theta}\left[\partial^{\mu}\left(A_{\mu}^{a}+\frac{1}{g} D_{\mu} \theta^{a}-\omega^{a}(x)\right)\right]=\frac{1}{g} \partial^{\mu} D_{\mu}
$$

ao calcularmos o determinante desta expressão, culminaremos com um termo que pode ser identificado com a seguinte integral gaussiana,

$$
\operatorname{det}\left(\frac{1}{g} \partial^{\mu} D_{\mu}\right)=\int[d c][d \bar{c}] e^{i \int d^{4} x \bar{c}\left(-\partial^{\mu} D_{\mu}\right) c} .
$$

Para que a identidade acima seja válida, esses campos $c$ e $\bar{c}$ devem ser anticomutantes, porém eles são escalares, e consecutivamente possuem spin nulo. Embora este fato viole a relação spin-estatística, não haverá problema algum na utilização desses campos, já que eles não representam nenhuma partícula real. Além disto, o comportamento atípico destes campos fez com que fossem batizados por ghosts de Faddeev-Popov.

A relação (3.42), introduzirá um novo termo na lagrangiana de Yang-Mills, a saber

$$
\mathcal{L}_{\text {ghost }}=\bar{c}\left(-\partial^{\mu} D_{\mu}\right) c=-\bar{c}^{\mathrm{a}} \partial^{2} \delta^{a c} c^{c}-g \bar{c}^{\mathrm{a}} \partial^{\mu} f^{a b c} A_{\mu}^{b} c^{c}
$$

O primeiro destes fatores da lagrangiana dos ghosts, representa o termo cinético dos campos $c$, e a partir dele podemos obter o propagador para os ghosts,

$$
\left\langle c^{a}(x) \bar{c}^{b}(y)\right\rangle=\int \frac{d^{4} k}{(2 \pi)^{4}} \frac{i}{k^{2}} \delta^{a b} e^{-i k(x-y)},
$$

já o segundo fator, representa uma interação entre os ghosts e os bósons de calibre.

\subsubsection{Interações na teoria de Yang-Mills}

Até o presente momento, já definimos os propagadores para os férmions, bósons de calibre, e ghosts, que estão representados em (3.45a), (3.45b) e (3.45c), respectivamente.

$$
\longleftarrow=\frac{i}{\not k-m}
$$

$\sim_{a, \mu}^{m}=\frac{-i \delta^{a b}}{k^{2}}\left(g^{\mu \nu}-(1-\xi) \frac{k^{\mu} k^{\nu}}{k^{2}}\right)$

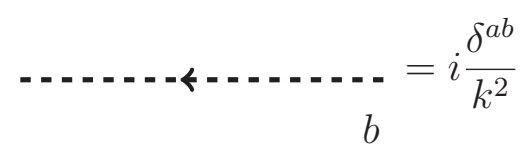


Desta forma, para definirmos o restante das regras de Feynman, ainda é necessário estudar as interações que se dão entre os bósons de calibre e os férmions, entre esses bósons e os ghosts, e por último os dois tipos de auto-interação destes bósons.

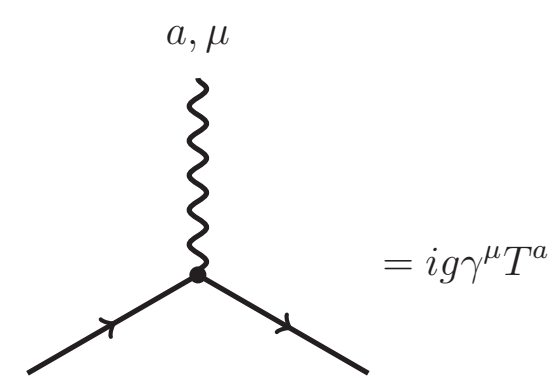

Posto isto, começaremos analisando o termo de interação entre os bósons de calibre e os férmions, cujo diagrama se encontra em (3.46). Este vértice de interação depende apenas da constante de acoplamento $g$, de uma matriz gama, e de um dos geradores do grupo $S U(N)$. Portanto, embora sua estrutura dependa da representação do grupo de simetria e do caráter espinorial dos campos fermiônicos, após a soma nos índices de Dirac e nos índices de cor, sua contribuição resultará em uma constante multiplicativa.

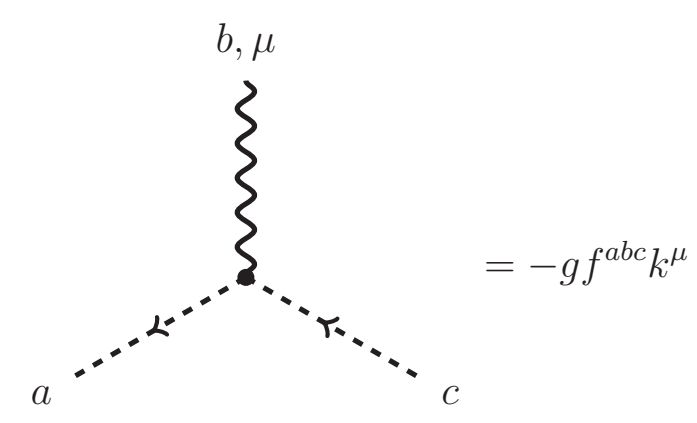

Em contrapartida, o vértice de interação entre os bósons de calibre e os ghosts, além de depender da constante de estrutura, depende também dos momentos dos ghosts. Ademais, o diagrama representando esta interação consta em (3.47), na qual podemos observar a existência de uma seta nas pernas dos ghosts, cuja função é indicar o sentido do fluxo fantasmagórico.

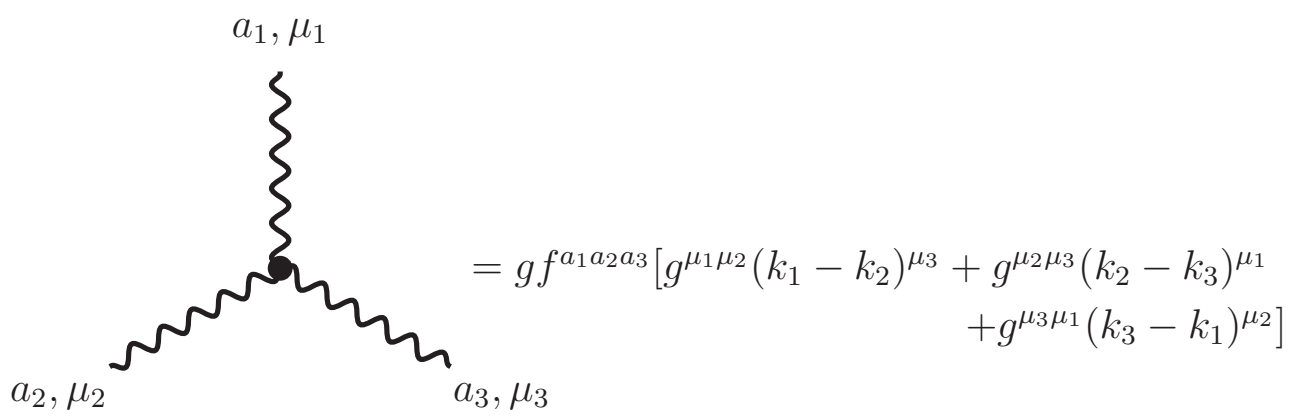

Agora, nos concentrando nas auto-interações dos bósons de calibre, nota-se em (3.48), que o vértice com 3 pontos, isto é, aquele que representa a interação gerada pelo 
termo proporcional à terceira potência do campo $A$, assim como o vértice de interação com os ghosts, depende de uma combinação dos momentos participantes do vértice. Essa similaridade não é a toa, pois como já foi dito, a nossa representação das teorias de calibre possui uma quantidade de graus de liberdade maior do que a existente, e os ghosts surgem justamente para cancelar esses graus de liberdade espúrios.

Por fim, a auto-interação de 4 pontos, que é gerada pelo termo em $\mathcal{L}_{B}$ contendo quatro potências de $A$, depende apenas de constantes de estrutura, e de combinações de métricas do espaço-tempo, o que acarreta, após todas as somas nos índices de cor e contrações dos índices de Lorentz, em uma constante multiplicativa. A priori, $\mathcal{L}_{B}$ gera 16 termos proporcionais à $A^{4}$, porém, utilizando a antissimetria de $f^{a b c}$, é possível agrupa-los em grupos de 4 termos, e assim culminamos com a combinação descrita em (3.49).

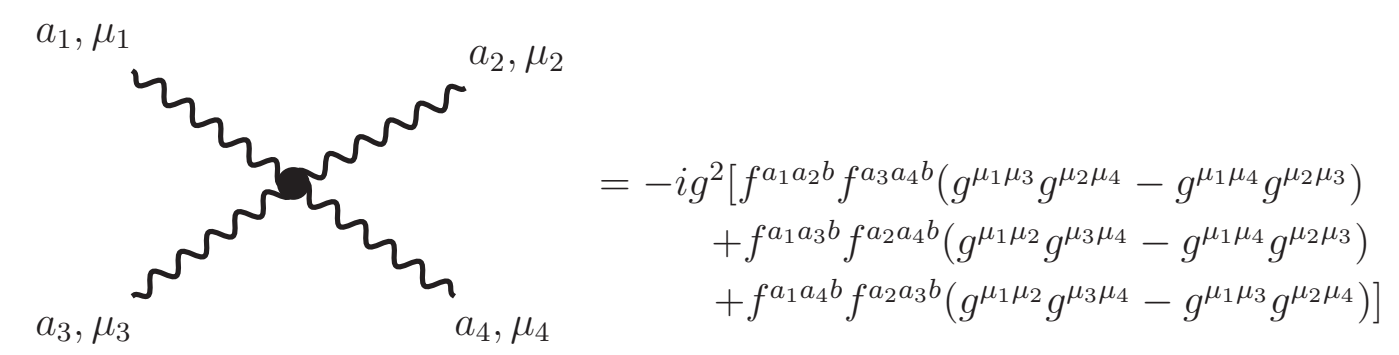

\subsection{Amplitudes frontais da teoria de Yang-Mills}

Até o presente momento, descrevemos as regras de Feynman para a teoria de Yang-Mills à temperatura nula, e assim, para incluir os efeitos da temperatura, iremos seguir a prescrição descrita em 2.3.

De maneira prática, a substituição $k_{0} \rightarrow i \omega_{n}$ não será necessária, pois como mostramos em (2.74), ao calcular a soma nas frequências de Matsubara através dos resíduos da cotangente/tangente hiperbólica, retornamos automaticamente ao caso em que $k_{0}$ assume valores contínuos.

Além disso, já visando a descrição de um plasma formado por bósons de calibre e férmions, tornaremos nossa atenção às correções dos propagadores livres apresentadas no apêndice B, sendo que estas têm como função incluir os efeitos provenientes das interações entre uma partícula, que se propaga livremente, com aquelas partículas advindas das flutuações do vácuo. Para entender melhor este processo, basta acompanhar a análise feita para as interações da ordem de um e dois loops representadas nos diagramas presentes em (B.17) e (B.21), respectivamente.

Todavia, para um sistema com temperatura finita, além dessa interferência na propagação de uma partícula gerada pelo vácuo, teremos também uma resistência criada pelas partículas pertencentes ao ensemble formador do plasma. Isto é, imagine que a 
partícula, a qual o propagador refere-se, pertence a um conjunto de várias outras, assim formando um plasma, e este por sua vez gera um banho térmico que dificulta a propagação das partículas em seu interior.

\subsubsection{Auto-energias da teoria de Yang-Mills}

Agora, levando em consideração apenas os diagramas da ordem de um loop em (B.22), calcularemos as correções para os propagadores dos férmions, dos bósons de calibre e dos ghosts de Faddeev e Popov.

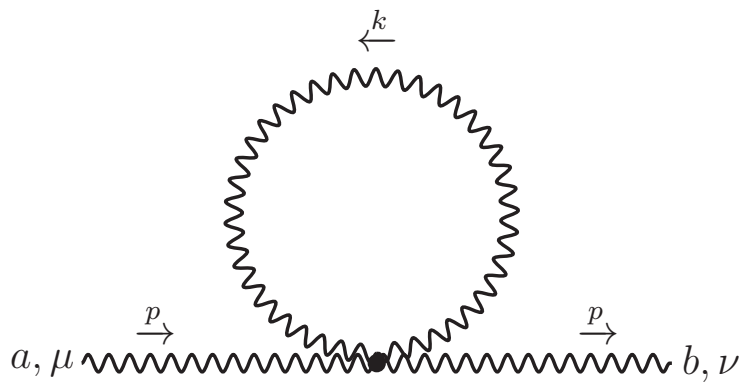

(a)

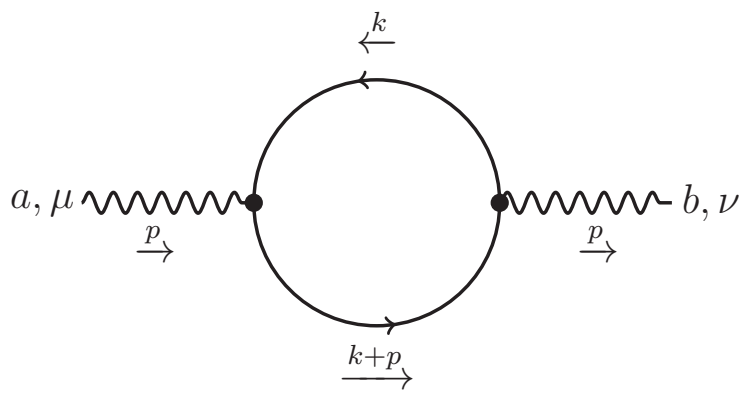

(c)

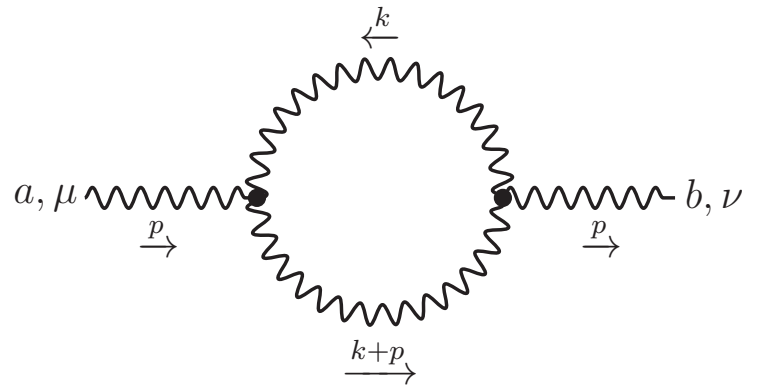

(b)

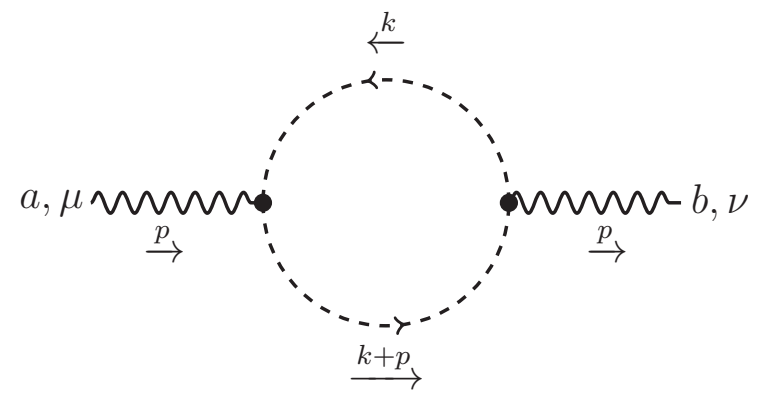

(d)

Figura 7 - Compontes da auto-energia dos bósons de calibre, cujas interações associadas são: (a) auto-interação de 4 pontos dos bósons de calibre; (b) auto-interação de 3 pontos dos bósons de calibre ; (c) interação entre bósons de calibre e férmions; e (d) interação entre bósons de calibre e ghosts.

Posto isto, iniciaremos com as correções para os bósons de calibre, que incluem dois loops gerados pelos termos de auto-interação com 3 e 4 pontos, e outros dois gerados pelos termos de interação com os férmions e com os ghosts, conforme figura 7.

Primeiramente, para o termo de interação com 4 pontos, temos

$$
\Pi_{T \mu \nu}^{a b}=\frac{1}{2 !} T \sum_{n} \int \frac{d^{D-1} k}{(2 \pi)^{D-1}}(-i) g^{2} W_{\mu \nu \lambda_{1} \lambda_{2}}^{a b c_{1} c_{2}}(-i) \delta_{c_{1} c_{2}} \frac{d^{\lambda_{1} \lambda_{2}}(k)}{k^{2}},
$$

em que $d^{\lambda \rho}(k)=g^{\lambda \rho}-(1-\xi) \frac{k^{\mu} k^{\nu}}{k^{2}}$ e $W_{\mu \nu \lambda \rho}^{a b c d}=\left(f^{a c e} f^{b d e}-f^{a d e} f^{c b e}\right) g_{\mu \nu} g_{\lambda \rho}+\left(f^{a b e} f^{c d e}-\right.$ $\left.f^{a d e} f^{b c e}\right) g_{\mu \lambda} g_{\nu \rho}+\left(f^{a c e} f^{d b e}-f^{a b e} f^{c d e}\right) g_{\mu \rho} g_{\lambda \nu}$. 
Como podemos ver na figura $7 \mathrm{a}$, o loop para este termo contém apenas um ponto conectado à duas pernas externas, o que faz essa estrutura assemelhar-se à (2.61). E assim como ocorreu para o tadpole, a sua expressão em amplitudes frontais resulta em um diagrama de arvore contendo apenas um único vértice de interação.

$$
\Pi_{T \mu \nu}^{a b}=-\frac{1}{2} \int \frac{d^{D-1} k}{(2 \pi)^{D-1}} \frac{N_{B}(\beta|\vec{k}|)}{2|\vec{k}|}(-i) \delta_{c_{1} c_{2}} d^{\lambda_{1} \lambda_{2}}(k)\left[\begin{array}{c}
a, \mu \\
c_{1}, \lambda_{1}^{n_{c_{2}}, \lambda_{2}}
\end{array}\right]_{k^{2}=0}
$$

Adicionalmente, se caso utilizarmos o calibre de Feynman-'t Hooft, o integrando da expressão acima será independente da parte angular dos momentos, e teremos apenas que integrar uma potência inteira de $|\vec{k}|$ multiplicando a distribuição de Bose-Einstein.

Embora os outros diagramas da figura 7, também contenham duas pernas externas, eles possuem um vértice de interação a mais. Todavia, mesmo com essa diferença, as contribuições de todos os diagramas da figura 7 são da mesma ordem, pois o vértice de 4 pontos é proporcional à $g^{2}$, enquanto os outros são proporcionais à $g$.

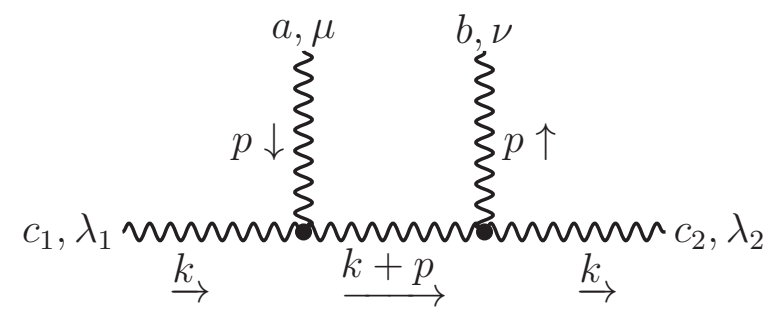

(a)

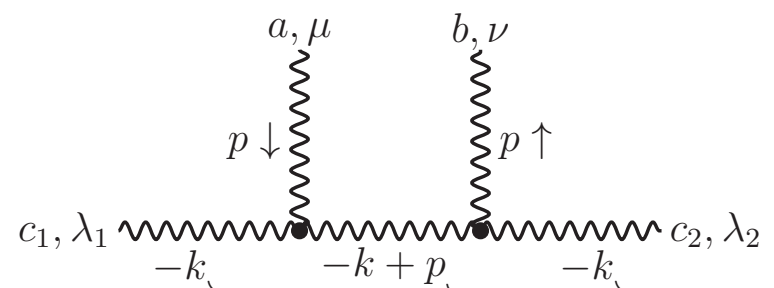

(c)

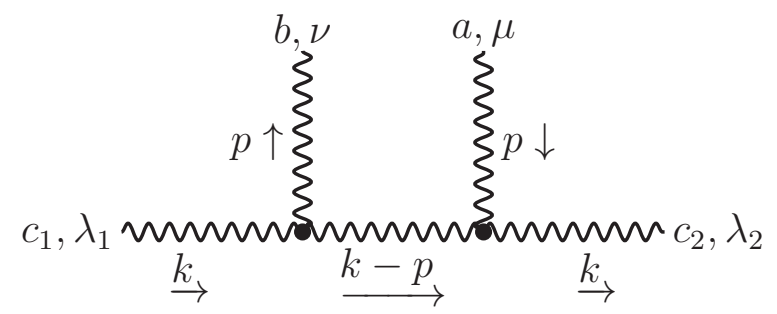

(b)

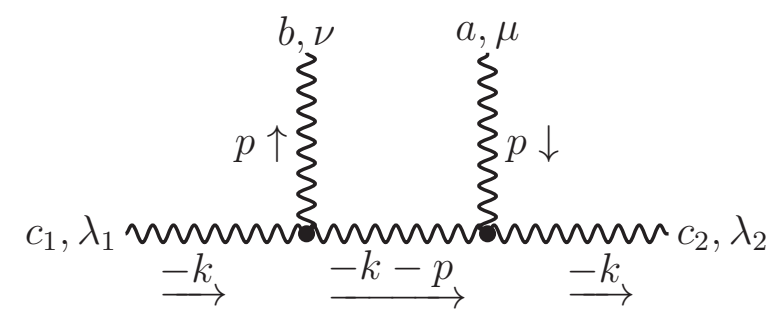

(d)

Figura 8 - Amplitudes frontais, com auto-interações de 3 pontos, da auto-energia dos bósons de calibre.

Dando prosseguimento, o diagrama com interações de 3 pontos representado na 
figura 7b, irá contribuir com

$$
\begin{aligned}
\Pi_{G \mu \nu}^{a b}=\frac{1}{2 !} T \sum_{n} \int \frac{d^{D-1} k}{(2 \pi)^{D-1}} & \left(g f^{b c_{1} c_{4}} V_{\nu \lambda_{1} \lambda_{4}}(-p,-k, k+p)(-i) \delta_{c_{1} c_{2}} \frac{d^{\lambda_{1} \lambda_{2}}(k)}{k^{2}}\right. \\
& \left.\times g f^{a c_{3} c_{2}} V_{\mu \lambda_{3} \lambda_{2}}(p,-k-p, k)(-i) \delta_{c_{3} c_{4}} \frac{d^{\lambda_{3} \lambda_{4}}(k+p)}{(k+p)^{2}}\right),
\end{aligned}
$$

sendo $V_{\mu \lambda \rho}(k, q, p)=(k-q)_{\rho} g_{\mu \lambda}+(p-k)_{\lambda} g_{\rho \mu}+(q-p)_{\mu} g_{\lambda \rho}$. Após efetuada a somatória em $n$, obteremos as amplitudes frontais da figura 8, cuja expressão quantitativa pode ser escrita como

$$
\begin{aligned}
\Pi_{G \mu \nu}^{a b}= & -\frac{1}{2} \int \frac{d^{D-1} k}{(2 \pi)^{D-1}} \frac{N_{B}(\beta|\vec{k}|)}{2|\vec{k}|}(-i) \delta_{c_{1} c_{2}} \frac{d^{\lambda_{1} \lambda_{2}}(k)}{2}( \\
& g f^{b d_{1} c_{2}} V_{\nu \sigma_{1} \lambda_{2}}(-p, k+p,-k)(-i) \delta_{d_{1} d_{2}} \frac{d^{\sigma_{1} \sigma_{2}}(k+p)}{(k+p)^{2}} g f^{a c_{1} d_{2}} V_{\mu \lambda_{1} \sigma_{2}}(p, k,-k-p)+ \\
& g f^{a d_{1} c_{2}} V_{\mu \sigma_{1} \lambda_{2}}(p, k-p,-k)(-i) \delta_{d_{1} d_{2}} \frac{d^{\sigma_{1} \sigma_{2}}(k-p)}{(k-p)^{2}} g f^{b c_{1} d_{2}} V_{\nu \lambda_{1} \sigma_{2}}(-p, k,-k+p)+ \\
& g f^{b d_{1} c_{2}} V_{\nu \sigma_{1} \lambda_{2}}(-p,-k+p, k)(-i) \delta_{d_{1} d_{2}} \frac{d^{\sigma_{1} \sigma_{2}}(-k+p)}{(-k+p)^{2}} g f^{a c_{1} d_{2}} V_{\mu \lambda_{1} \sigma_{2}}(p,-k, k-p)+ \\
& \left.g f^{a d_{1} c_{2}} V_{\mu \sigma_{1} \lambda_{2}}(p,-k-p, k)(-i) \delta_{d_{1} d_{2}} \frac{d^{\sigma_{1} \sigma_{2}}(-k-p)}{(-k-p)^{2}} g f^{b c_{1} d_{2}} V_{\nu \lambda_{1} \sigma_{2}}(-p,-k, k+p)\right)_{k^{2}=0}
\end{aligned}
$$

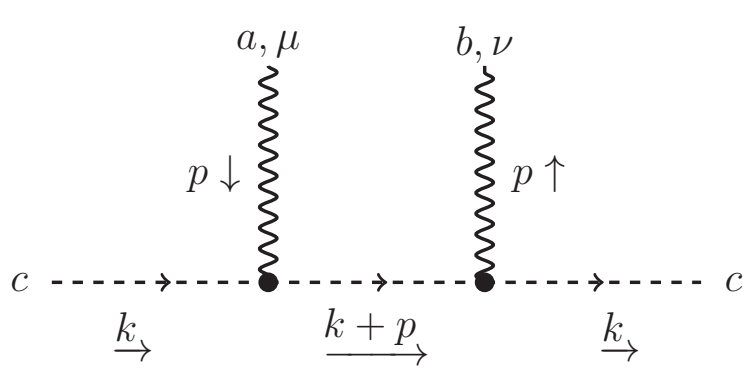

(a)

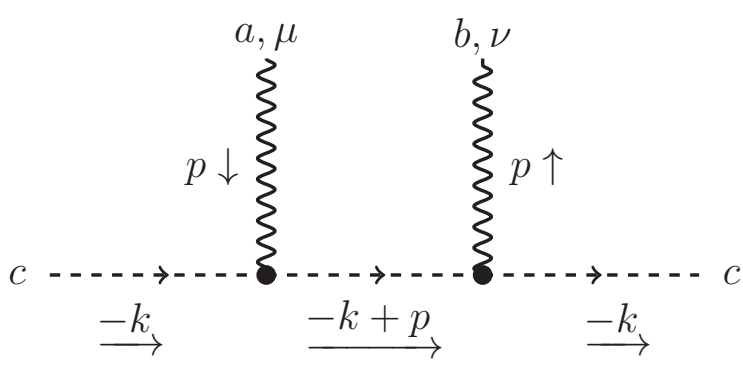

(c)

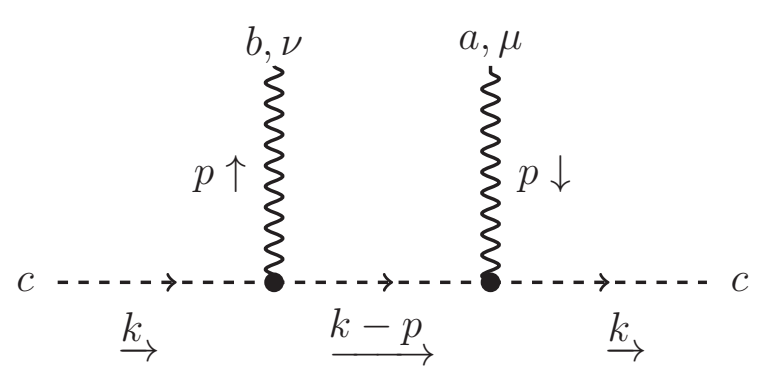

(b)

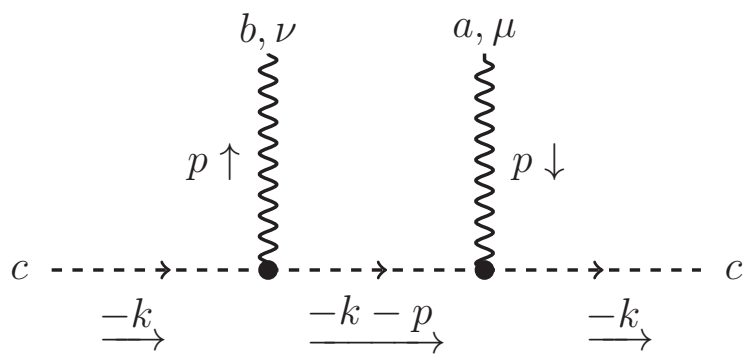

(d)

Figura 9 - Amplitudes frontais, com interações entre bósons de calibre e ghosts, da autoenergia dos bósons de calibre. 
Como já foi adiantado, incluídos nestes fatores acima, estão alguns graus de liberdade espúrios, e para lidar com eles, vamos calcular a correção para os propagadores dos bósons de calibre via interação com os ghosts, figura $7 \mathrm{~d}$.

$$
\Pi_{F P \mu \nu}^{a b}=-T \sum_{n} \int \frac{d^{D-1} k}{(2 \pi)^{D-1}}\left[-g f^{c_{4} b c_{1}}(k+p)_{\nu} \frac{i \delta_{c_{1} c_{2}}}{k^{2}}(-1) g f^{c_{2} a c_{3}} k_{\mu} \frac{i \delta_{c_{3} c_{4}}}{(k+p)^{2}}\right]
$$

Essa expressão resultará nas amplitudes frontais representadas pelos diagramas na figura 9, que culminam na seguinte correção para $\Pi_{\mu \nu}^{a b}$ :

$$
\begin{gathered}
\Pi_{F P \mu \nu}^{a b}=\int \frac{d^{D-1} k}{(2 \pi)^{D-1}} \frac{N_{B}(\beta|\vec{k}|)}{2|\vec{k}|} \frac{i}{2}\left(-g f^{c b d_{1}}(k+p)_{\nu} \frac{i \delta_{d_{1} d_{2}}}{(k+p)^{2}}(-1) g f^{d_{2} a c} k_{\mu}+\right. \\
-g f^{c a d_{1}}(k-p)_{\nu} \frac{i \delta_{d_{1} d_{2}}}{(k-p)^{2}}(-1) g f^{d_{2} b c} k_{\mu}-g f^{c b d_{1}}(-k+p)_{\nu} \frac{i \delta_{d_{1} d_{2}}}{(-k+p)^{2}}(-1) g f^{d_{2} a c}\left(-k_{\mu}\right)+ \\
\left.-g f^{c a d_{1}}(-k-p)_{\nu} \frac{i \delta_{d_{1} d_{2}}}{(-k-p)^{2}}(-1) g f^{d_{2} b c}\left(-k_{\mu}\right)\right)_{k^{2}=0}
\end{gathered}
$$

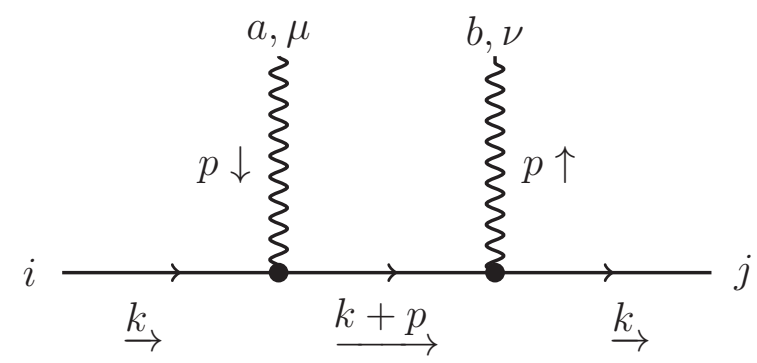

(a)

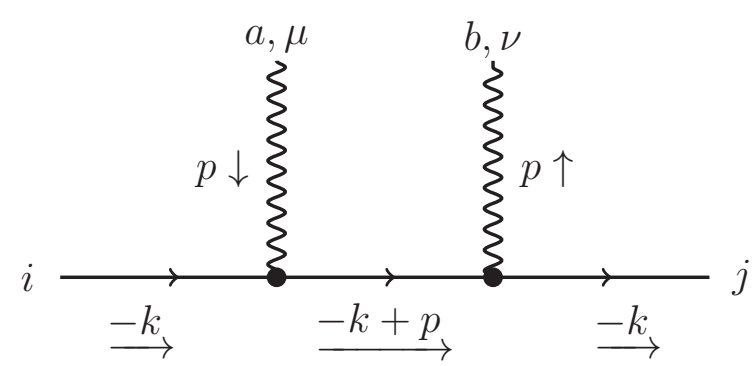

(c)

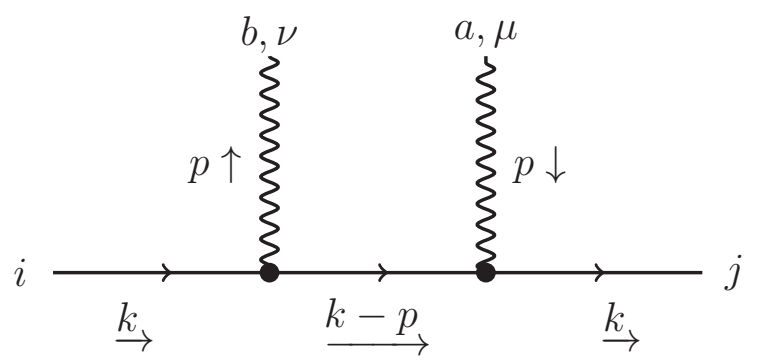

(b)

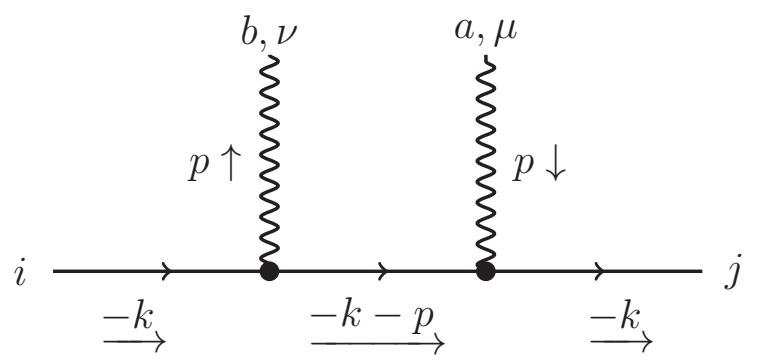

(d)

Figura 10 - Amplitudes frontais, com interações entre bósons de calibre e férmions, da auto-energia dos bósons de calibre.

Por fim, calcularemos a única correção que contém um loop fermiônico, figura 7c, e consecutivamente, será também a única com uma função de Fermi-Dirac no integrando.

$$
\Pi_{F \mu \nu}^{a b}=-N_{f} T \sum_{n} \int \frac{d^{D-1} k}{(2 \pi)^{D-1}} \operatorname{Tr}\left[i g \gamma_{\mu} T^{a} \frac{i}{\not k+\not p-m} i g \gamma_{\nu} T^{b} \frac{i}{\not k-m}\right]
$$


As amplitudes frontais geradas por esta expressão, figura 10, não mais estarão na camada de massa nula, pois como o loop é fermiônico, a camada de massa será relativa aos férmions, e consequentemente $k^{2}=m^{2}$.

$$
\begin{aligned}
& \Pi_{F \mu \nu}^{a b}=-N_{f} \int \frac{d^{D-1} k}{(2 \pi)^{D-1}} \frac{N_{F}\left(\beta \omega_{k}\right)}{2 \omega_{k}} \frac{1}{2} \operatorname{Tr}\left(\left[i g \gamma_{\nu} T^{b} \frac{i}{\not k+\not p-m} i g \gamma_{\mu} T^{a}+i g \gamma_{\mu} T^{a} \frac{i}{\not k-\not p-m} i g \gamma_{\nu} T^{b}\right]\right. \\
& \left.\times i(\not k+m)+\left[i g \gamma_{\nu} T^{b} \frac{i}{-\not k+\not p-m} i g \gamma_{\mu} T^{a}+i g \gamma_{\mu} T^{a} \frac{i}{-\not k-\not p-m} i g \gamma_{\nu} T^{b}\right] i(-\not k+m)\right)_{k^{2}=m^{2}}
\end{aligned}
$$

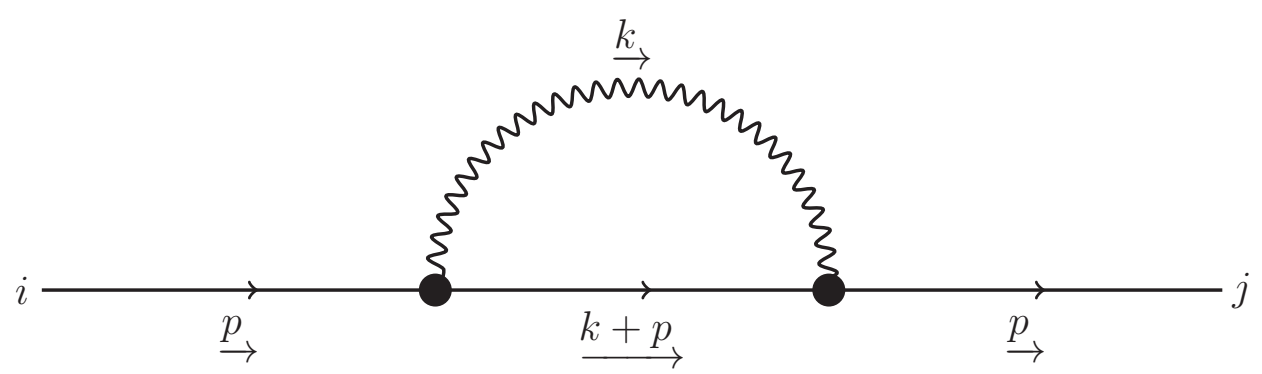

Figura 11 - Auto-energia dos férmions.

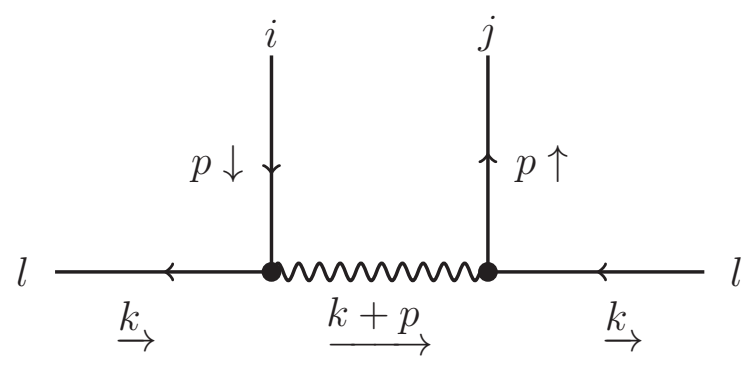

(a)

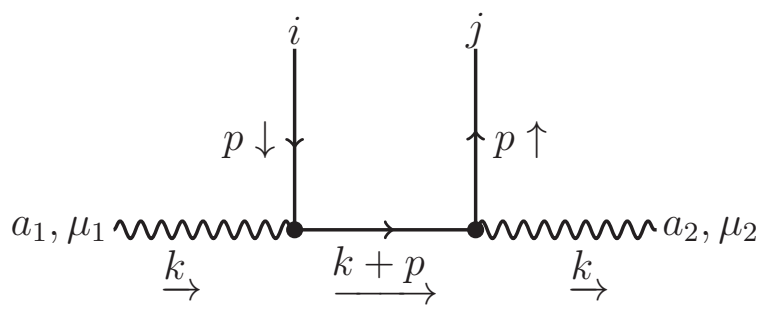

(c)

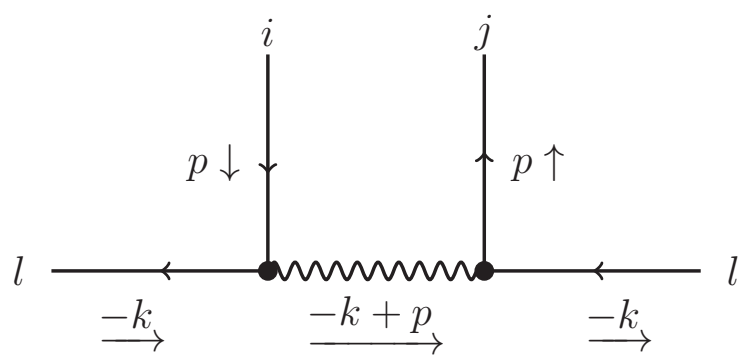

(b)

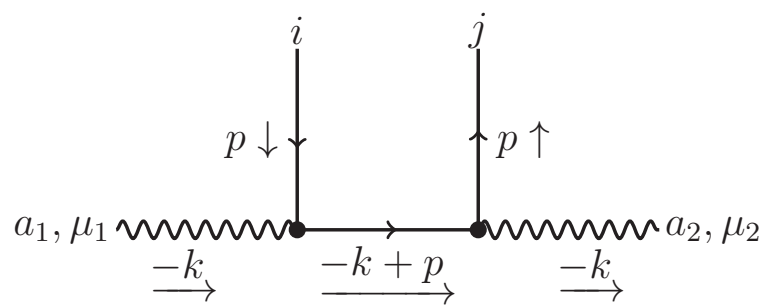

(d)

Figura 12 - Amplitudes frontais, com interações entre bósons de calibre e férmions, da auto-energia dos férmions.

Nesses cálculos para os bósons de calibre, só ocorreram loops com um tipo de campo, e consecutivamente um único tipo de estatística. Agora, para o caso dos férmions, podemos notar na figura 11, que o loop envolve dois tipos de campos, e assim dois tipos de 
distribuição estatística. Desta forma, as correções de um loop para os férmions culminam em

$$
\begin{aligned}
\Sigma_{i j} & =T \sum_{n} \int \frac{d^{D-1} k}{(2 \pi)^{D-1}}\left[i g \gamma_{\mu} T_{i l}^{a} \frac{i}{\not k+\not p-m} i g \gamma_{\nu} T_{l j}^{b}(-i) \delta_{a b} \frac{d^{\mu \nu}(k)}{k^{2}}\right] \\
& =-\int \frac{d^{D-1} k}{(2 \pi)^{D-1}} \frac{1}{2}\left[\frac { N _ { B } ( \beta | \vec { k } | ) } { 2 | \vec { k } | } ( - i ) \delta _ { a _ { 1 } a _ { 2 } } d ^ { \mu _ { 1 } \mu _ { 2 } } ( k ) \left(i g \gamma_{\mu_{1}} T_{i l}^{a_{1}} \frac{i}{\not k+\not p-m} i g \gamma_{\mu_{2}} T_{l j}^{a_{2}}+i g \gamma_{\mu_{1}} T_{i l}^{a_{1}} \times\right.\right. \\
& \left.\times \frac{i}{-\not k+\not p-m} i g \gamma_{\mu_{2}} T_{l j}^{a_{2}}\right)_{k^{2}=0}-\frac{N_{F}\left(\beta \omega_{k}\right)}{2 \omega_{k}}\left(i(\not k+m) i g \gamma_{\mu_{1}} T_{i l}^{a_{1}}(-i) \delta_{a_{1} a_{2}} \frac{d^{\mu_{1} \mu_{2}}(k+p)}{(k+p)^{2}} i g \gamma_{\mu_{2}} T_{l j}^{a_{2}}+\right. \\
& \left.\left.+i(-\not k+m) i g \gamma_{\mu_{1}} T_{i l}^{a_{1}}(-i) \delta_{a_{1} a_{2}} \frac{d^{\mu_{1} \mu_{2}}(-k+p)}{(-k+p)^{2}} i g \gamma_{\mu_{2}} T_{l j}^{a_{2}}\right)_{k^{2}=m^{2}}\right] .
\end{aligned}
$$

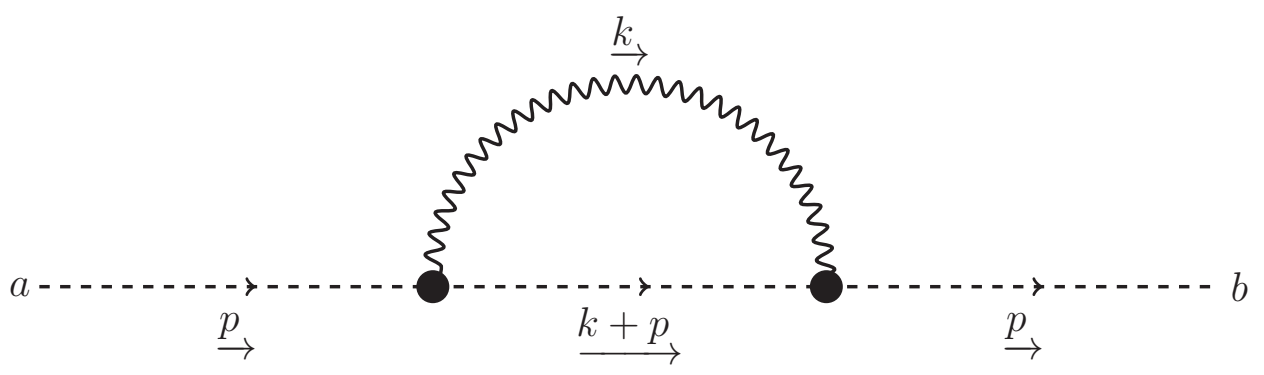

Figura 13 - Auto-energia dos ghosts.

Aliás, as amplitudes frontais da figura 12, contidas na expressão (3.58), são bem distintas, uma vez que seus propagadores representam diferentes tipos de campos. Adicionalmente, os momentos internos dos diagramas 12c e 12d, estão na camada de massa, enquanto que os campos dos diagramas 12a e 12b não possuem massa de repouso.

Além disso, outro loop que também envolve propagadores de dois tipos diferentes de campos, é o relativo as correções para os propagadores dos ghosts, figura 13. Porém, diferentemente do caso dos férmions, a estatística dos propagadores nas amplitudes frontais 


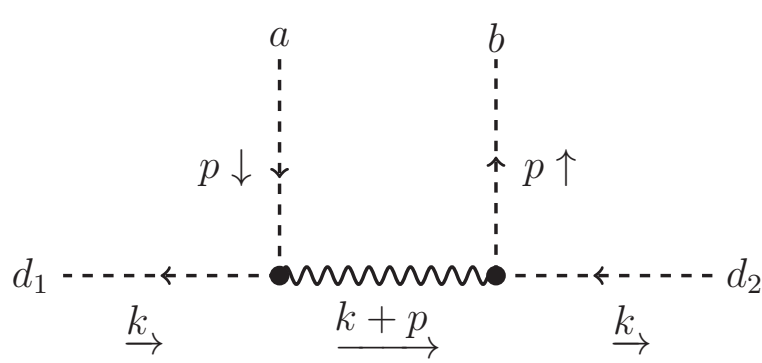

(a)

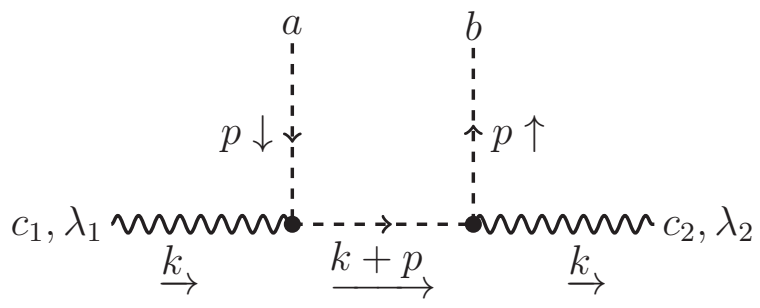

(c)

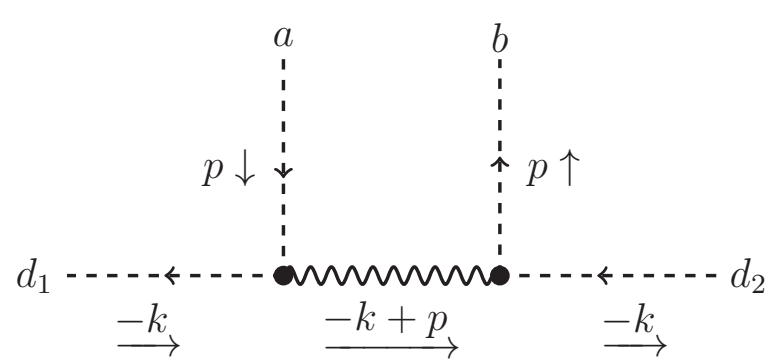

(b)

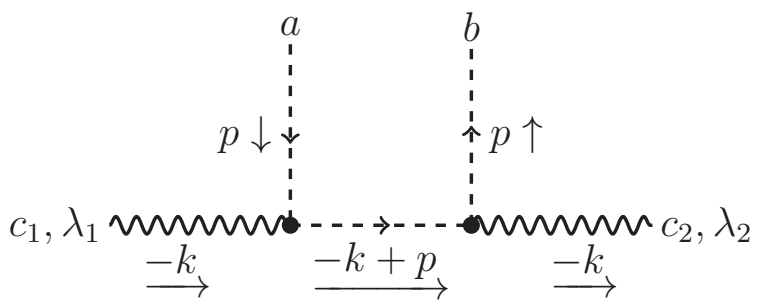

(d)

Figura 14 - Amplitudes frontais, com interações entre bósons de calibre e ghosts, da auto-energia dos ghosts.

se mantêm.

$$
\begin{aligned}
& \tilde{\Pi}^{a b}=T \sum_{n} \int \frac{d^{D-1} k}{(2 \pi)^{D-1}}\left[(-g) f^{d_{1} c_{1} b}(k+p)_{\lambda_{1}}(-i) \delta_{c_{1} c_{2}} \frac{d^{\lambda_{1} \lambda_{2}}(k)}{k^{2}}(-g) f^{a c_{2} d_{2}} k_{\lambda_{2}} \frac{i \delta^{d_{1} d_{2}}}{(k+p)^{2}}\right] \\
& =-\int \frac{d^{D-1} k}{(2 \pi)^{D-1}} \frac{N_{B}(\beta|\vec{k}|)}{2|\vec{k}|} \frac{1}{2}\left[( - i ) \delta _ { c _ { 1 } c _ { 2 } } d ^ { \lambda _ { 1 } \lambda _ { 2 } } ( k ) \left((-g) f^{a c_{1} d_{1}} k_{\lambda_{1}} \frac{i \delta^{d_{1} d_{2}}}{(k+p)^{2}}(-g) f^{d_{2} c_{2} b}(k+p)_{\lambda_{2}}+\right.\right. \\
& \left.+(-g) f^{a c_{1} d_{1}}\left(-k_{\lambda_{1}}\right) \frac{i \delta^{d_{1} d_{2}}}{(-k+p)^{2}}(-g) f^{d_{2} c_{2} b}(-k+p)_{\lambda_{2}}\right)+i \delta_{d_{1} d_{2}}\left((-g) f^{a c_{1} d_{1}} k_{\lambda_{1}}(-i) \delta_{c_{1} c_{2}} \times\right. \\
& \left.\left.\times \frac{d^{\lambda_{1} \lambda_{2}}(k+p)}{(k+p)^{2}}(-g)(k+p)_{\lambda_{2}}+(-g) f^{a c_{1} d_{1}}\left(-k_{\lambda_{1}}\right)(-i) \delta_{c_{1} c_{2}} \frac{d^{\lambda_{1} \lambda_{2}}(-k+p)}{(-k+p)^{2}}(-g)(-k+p)_{\lambda_{2}}\right)\right]_{k^{2}=0}
\end{aligned}
$$

Por fim, embora as amplitudes frontais das figuras 14 e 12, se separem em dois grupos distintos, elas ainda obedecem as regras estabelecidas na seção 2.3.2 do capítulo anterior, pois é evidente que esses diagramas configuram um conjunto completo de permutações cíclicas. 



\section{Limite de altas temperaturas da TQFT}

Na seção 2.3 vimos que as amplitudes térmicas, em ordem de um loop, possuem a seguinte forma genérica:

$$
\int \frac{d^{D-1} k}{(2 \pi)^{D-1}} \frac{N_{B, F}\left(\beta \omega_{k}\right)}{2 \omega_{k}}[\text { Amplitudes Frontais]. }
$$

A seguir, veremos como é possível obter essas amplitudes frontais, inclusive para o caso de uma teoria de calibre não-abeliana, em um espaço-tempo de dimensão $D$, com grupo de simetria $S U(N)$.

Assim, para concluir os cálculos das amplitudes térmicas, bastaria efetuar um último passo, que consiste em solucionar as integrais espaciais em (4.1), porém a maioria dessas integrais não possuem forma fechada, para valores arbitrários da temperatura. Veremos que no caso de particular interesse para o presente estudo, a saber, altas temperaturas, as amplitudes frontais simplificam-se consideravelmente.

Para iniciarmos a análise com um caso simples, consideremos o tadpole da teoria $\lambda \phi^{4}$. A partir da expressão (B.18), generalizada para $D$ dimensões espaço-temporais, no limite de massa nula, temos

$$
\Pi_{1}=12 \lambda \int \frac{d^{D-1} k}{(2 \pi)^{D-1}} \frac{1}{|\vec{k}|} \frac{1}{e^{\beta|\vec{k}|}-1},
$$

cuja solução fechada existe e será calculada a seguir.

A fim de solucionar as integrais em (4.2), começaremos explorando a ausência de termos com dependência angular no seu integrando que, juntamente com uma mudança do sistema de coordenadas, simplifica a expressão acima em

$$
\Pi_{1}=12 \lambda \frac{1}{(2 \pi)^{D-1}} \int d \Omega_{D-1} \int d|\vec{k}||\vec{k}|^{D-3} \frac{1}{e^{\beta|\vec{k}|}-1} .
$$

Aqui vemos duas integrações distintas, sendo que a dependência angular ficou restrita à integral definida pela superfície esférica de $D-1$ dimensões, cuja contribuição será apenas uma constante dependente da dimensão espacial, e que pode ser obtida através do seguinte artifício (PESKIN; SCHROEDER, 1995):

$$
\begin{aligned}
(\sqrt{\pi})^{d} & =\left(\int d x e^{-x^{2}}\right)^{d}=\left(\int d x_{1} e^{-x_{1}^{2}}\right)\left(\int d x_{2} e^{-x_{2}^{2}}\right) \ldots\left(\int d x_{d} e^{-x_{d}^{2}}\right) \\
& =\int d x_{1} \ldots d x_{d} e^{-\sum_{i=1}^{d} x_{i}^{2}} \stackrel{\text { coordenadas esféricas }}{\longrightarrow} \int d \Omega_{d} \int d \rho \rho^{d-1} e^{-\rho^{2}} \\
& =\left(\int d \Omega_{d}\right) \frac{1}{2} \int_{0}^{\infty} d\left(\rho^{2}\right)\left(\rho^{2}\right)^{\frac{d}{2}-1} e^{-\left(\rho^{2}\right)}=\frac{1}{2} \Gamma\left(\frac{d}{2}\right) \int d \Omega_{d} .
\end{aligned}
$$


Na última passagem acima, reconhecemos que a integral em $\rho^{2}$ é uma das representações da função gama. Além disso, ao isolarmos a integral da superfície esférica neste resultado, temos

$$
\int \Omega_{D-1}=\frac{2 \pi^{\frac{D-1}{2}}}{\Gamma\left(\frac{D-1}{2}\right)}
$$

Já a solução da parte radial de (4.3) possui passos mais complexos e longos, e por isto, utilizaremos diretamente a seguinte fórmula (GRADSHTEYN; RYZHIK, 1980):

$$
\int_{0}^{\infty} d r \frac{r^{a}}{e^{\beta r}-1}=\left(\frac{1}{\beta}\right)^{a+1} \zeta(a+1) \Gamma(a+1)=T^{a+1} \zeta(a+1) \Gamma(a+1),
$$

em que $\zeta$ representa a função zeta de Riemann.

Desta forma, para obter a solução de (4.3), basta combinar os resultados (4.5) e $(4.6)$

$$
\Pi_{1}=\frac{3 \lambda T^{D-2} \zeta(D-2) \Gamma(D-2)}{2^{D-4} \pi^{\frac{D-1}{2}} \Gamma\left(\frac{D-1}{2}\right)} .
$$

Esse resultado, quando considerado para o caso usual, em que o espaço-tempo possui 4 dimensões, resulta em uma auto-energia proporcional à $\lambda T^{2}$. E quando substituído no propagador (B.7), culmina em

$$
D\left(\omega_{n}, \vec{k}\right)=\frac{1}{\omega_{n}^{2}+\vec{k}^{2}+m^{2}+\lambda T^{2}} .
$$

Aqui vemos, que essa correção, $\Pi_{1}$, somente será significativa quando $k^{2} \lesssim \lambda T^{2}$, ou alternativamente, pensando nas componentes do quadrimomento $k$ separadamente, $|\vec{k}| \lesssim \sqrt{\lambda} T$. Por outro lado, quando $|\vec{k}| \gtrsim T$, podemos desprezar a correção advinda da auto-energia, e utilizar o propagador livre.

Além dessas condições expostas no parágrafo anterior, é possível notar em (4.2), que a função de distribuição dentre seu papel de amortizar os valores do integrando, é menos eficaz quando $|\vec{k}| \sim T$, isto é, o integrando gera suas maiores contribuições, quando os momentos internos de um loop são da ordem da temperatura.

Dito isso, como alternativa para obtenção de uma solução das integrais em (4.1), parece ser apropriado utilizarmos a condição $|\vec{k}| \sim T$. Além disso, consideraremos também o limite de altas temperaturas, o que implica, consecutivamente, na condição $k \gg p_{i}$, em que $p_{i}$ representa todos os momentos externos.

Na literatura(PISARSKI, 1989), os momentos que obedecem a condição exposta no parágrado acima, são comumente chamados de hard moments, e em contrapartida, os momentos que satisfazem $|\vec{k}| \sim \sqrt{\lambda} T$ foram batizados como soft moments. Fisicamente, quando consideramos $k \gg p_{i}$, estamos em um range de energia no qual as interações mais 
significativas são as colisões elásticas, e portanto estamos muito próximos de um sistema clássico.

Logo, quando aplicarmos a condição de hard moments na expressão (4.1), obteremos um limite clássico para as amplitudes térmicas, e este por sua vez é referenciado na literatura como limite HTL.

\subsection{Limite de altas temperaturas para a teoria $\lambda \phi^{3}$}

Com o intuito de ilustrar os efeitos do limite HTL em amplitudes térmicas, utilizaremos a teoria $\lambda \phi^{3}$. A primeira ordem de $\Pi$ para esta teoria é muito semelhante a (4.7), pois ela possui valor exato quando desprezamos a massa, e por isto, a aplicação deste limite em $\Pi_{1}$ é desnecessária. Portanto, partiremos diretamente para o cálculo de $\Pi_{2}$, cuja expressão se escreve como:

$$
\Pi_{2}=-\lambda^{2} \int \frac{d^{3} k}{(2 \pi)^{3}} \frac{1}{|\vec{k}|} \frac{1}{e^{\beta|\vec{k}|}-1}\left[\frac{1}{(k+p)^{2}}+\frac{1}{(k-p)^{2}}\right] .
$$

Como próximo passo, devemos impor a condição de hard moment na expressão acima, que se dará através de uma expansão dos denominadores do integrando de (4.9),

$$
\frac{1}{(k \pm p)^{2}}=\frac{1}{p^{2} \pm 2 k \cdot p}=\frac{1}{2 k \cdot p}\left(\frac{1}{1 \pm \frac{p^{2}}{2 k \cdot p}}\right)=\frac{1}{2 k \cdot p}\left(1 \mp \frac{p^{2}}{2 k \cdot p}+\ldots\right)
$$

Em seguida, substituiremos essas expansões em (4.9), o que resultará em

$$
\Pi_{2}=\lambda^{2} \int \frac{d^{3} k}{(2 \pi)^{3}} \frac{1}{|\vec{k}|} \frac{1}{e^{\beta|\vec{k}|}-1} \frac{1}{k \cdot p} .
$$

A contração entre $k$ e $p$ ainda pode ser simplificada, já que $k \cdot p=k_{0} p_{0}-\vec{k} \cdot \vec{p}=$ $|\vec{k}| p_{0}-|\vec{k}||\vec{p}| \cos (\theta)$, em que $\theta$ é o ângulo formado entre as partes espaciais de $k$ e $p$. Reescrevendo a expressão acima em coordenadas esféricas e escolhendo $\vec{p}$ na direção da última componente de $k$, obtemos

$$
\begin{aligned}
\Pi_{2} & =-\frac{\lambda^{2}}{(2 \pi)^{3}} \int_{0}^{2 \pi} d \phi \int_{0}^{\infty} d|\vec{k}| \frac{1}{e^{\beta|\vec{k}|}-1} \int_{0}^{\pi} d \theta \frac{\operatorname{sen}(\theta)}{p_{0}-|\vec{p}| \cos (\theta)} \\
& =\frac{\lambda^{2}}{(2 \pi)^{2}} \frac{1}{|\vec{p}|} \ln \left(\frac{p_{0}-|\vec{p}|}{p_{0}+|\vec{p}|}\right) \int_{0}^{\infty} d|\vec{k}| \frac{1}{e^{\beta|\vec{k}|}-1} .
\end{aligned}
$$

É notável que a integral na parte radial de $k$ diverge, e por isto, não podemos obter um resultado fechado. A origem desta divergência pode ser facilmente identificada no limite de massa nula das partículas térmicas. Ou seja, está implícito que $T \gg m$. De qualquer forma, este exemplo serviu para mostrar, em um contexto simples, como é aplicada a condição $k \gg p$. 
Além disso, este exemplo demonstra um tipo de divergência comum na TQFT, que é a divergência infravermelha em teorias de massa nula. Como já comentamos, as funções de distribuição inibem a aparição das divergências ultravioletas, porém, elas introduzem esse novo tipo de divergência.

Adicionalmente, na QFT com temperatura nula, as divergências são tratadas, em geral, via algum dos vários métodos de renormalização. Porém, o problema das divergências infravermelhas, à temperatura finita, possui um significado distinto e requer outras abordagens. Em certos casos é possível somar uma infinidade de diagramas de Feynman, de forma a obter um resultado finito (BRANDT et al., 1993; KRAMMER; REBHAN; SCHULZ, 1995; BOYANOVSKY et al., 1999; KREUZER; REBHAN; SCHULZ, 1990).

\subsection{Limite de altas temperaturas para a teoria de Yang-Mills}

O uso da teoria $\lambda \phi^{3}$ na seção anterior, serviu como um modelo bem simples da aplicação do limite HTL. Em contraste, a teoria de calibre não-abeliana, que estamos tratando desde o segundo capítulo, possui várias estruturas complexas nos denominadores das amplitudes frontais.

Essas dificuldades inerentes à teoria de Yang-Mills serão tratadas via aplicação da condição de hard moments através de uma expansão em série de Laurent das amplitudes frontais, seguida de uma análise dimensional, na qual selecionaremos os termos de maior contribuição. De forma geral, este tipo de procedimento permite visualizar o formato dos termos referentes à uma segunda ordem de aproximação, embora não contemple todos eles.

\subsubsection{HTL aplicado à auto-energia dos bósons de calibre}

Antes de aplicarmos qualquer condição ou limite às expressões da auto-energia, será necessário efetuar todos os traços e soma dos índices de cor que ficaram por fazer. Após todas essas manipulações ${ }^{1}$, as auto-energias dos bósons de calibre resultam em:

$$
\Pi_{T \mu \nu}^{a b}=\frac{-i g^{2} N \delta^{a b}}{2} \int \frac{d^{D-1} k}{(2 \pi)^{D-1}} \frac{|\vec{k}|^{-1}}{e^{\beta|\vec{k}|}-1}\left[(2 D-2) g_{\mu \nu}\right]
$$

$$
1
$$

Foi adotada a seguinte condição de normalização para o traço das matrizes $N \times N$ na representação fundamental,

$$
\operatorname{Tr}\left[T^{a} T^{b}\right]=\frac{1}{2} \delta^{a b}
$$

Além dessa relação, também utilizamos:

$$
\left(T^{a} T^{a}\right)_{i j}=\frac{N^{2}-1}{2 N} \delta_{i j} \text { e } f^{a c d} f^{b c d}=N \delta^{a b} .
$$

E por fim, consideramos as dimensões dos espinores como sendo pares. 


$$
\begin{aligned}
\Pi_{G \mu \nu}^{a b}= & \frac{i g^{2} N \delta^{a b}}{2} \int \frac{d^{D-1} k}{(2 \pi)^{D-1}} \frac{|\vec{k}|^{-1}}{e^{\beta|\vec{k}|}-1}\left[\frac{2(2 D-3) k_{\mu} k_{\nu}+(2 D-3) k_{\mu} p_{\nu}+(2 D-3) k_{\nu} p_{\mu}}{2 k \cdot p\left(1+\frac{p^{2}}{2 k \cdot p}\right)}+\right. \\
+ & \left.\frac{(D-6) p_{\mu} p_{\nu}+g_{\mu \nu}\left(2 k \cdot p+5 p^{2}\right)}{2 k \cdot p\left(1+\frac{p^{2}}{2 k \cdot p}\right)}+k \rightarrow-k\right] \\
\prod_{F P \mu \nu}^{a b}= & \frac{-i g^{2} N \delta^{a b}}{2} \int \frac{d^{D-1} k}{(2 \pi)^{D-1}} \frac{|\vec{k}|^{-1}}{e^{\beta|\vec{k}|}-1}\left[\frac{2 k_{\mu} k_{\nu}+k_{\mu} p_{\nu}+k_{\nu} p_{\mu}}{2 k \cdot p\left(1+\frac{p^{2}}{2 k \cdot p}\right)}+k \rightarrow-k\right] \\
\prod_{F \mu \nu}^{a b}= & \frac{i g^{2} N_{f} \delta^{a b}}{2} 2^{\frac{D}{2}} \int \frac{d^{D-1} k}{(2 \pi)^{D-1}} \frac{\omega_{k}^{-1}}{e^{\beta \omega_{k}}+1}\left[\frac{2 k_{\mu} k_{\nu}+k_{\mu} p_{\nu}+k_{\nu} p_{\mu}-g_{\mu \nu} k \cdot p}{2 k \cdot p\left(1+\frac{p^{2}}{2 k \cdot p}\right)}+k \rightarrow-k\right]
\end{aligned}
$$

Aqui vemos uma grande semelhança na estrutura das expressões acima, que compõem a auto-energia dos bósons de calibre, porém, antes de somá-las, faremos as expansões de seus denominadores, assim como feito em (4.10).

$$
\begin{gathered}
\Pi_{G \mu \nu}^{a b}=\frac{i g^{2} N \delta^{a b}}{2} \int \frac{d^{D-1} k}{(2 \pi)^{D-1}} \frac{|\vec{k}|^{-1}}{e^{\beta|\vec{k}|}-1}\left[\frac{(2 D-3)}{k \cdot p}\left(k_{\mu} p_{\nu}+k_{\nu} p_{\mu}\right)-\frac{(2 D-3) p^{2}}{(k \cdot p)^{2}} k_{\mu} k_{\nu}+\right. \\
\left.-\frac{(D-6) p^{2}}{(k \cdot p)^{2}} p_{\mu} p_{\nu}-\frac{5 p^{4}}{2(k \cdot p)^{2}} g_{\mu \nu}+2 g_{\mu \nu}\right] \\
\prod_{F P \mu \nu}^{a b}=\frac{-i g^{2} N \delta^{a b}}{2} \int \frac{d^{D-1} k}{(2 \pi)^{D-1}} \frac{|\vec{k}|^{-1}}{e^{\beta|\vec{k}|}-1}\left[\frac{k_{\mu} p_{\nu}+k_{\nu} p_{\mu}}{k \cdot p}-\frac{p^{2}}{(k \cdot p)^{2}} k_{\mu} k_{\nu}\right] \\
\prod_{F \mu \nu}^{a b}=\frac{i g^{2} N_{f} \delta^{a b}}{2} 2^{\frac{D}{2}} \int \frac{d^{D-1} k}{(2 \pi)^{D-1}} \frac{\omega_{k}^{-1}}{e^{\beta \omega_{k}}+1}\left[\frac{k_{\mu} p_{\nu}+k_{\nu} p_{\mu}}{k \cdot p}-\frac{p^{2}}{(k \cdot p)^{2}} k_{\mu} k_{\nu}-g_{\mu \nu}\right]
\end{gathered}
$$

O termo do tadpole não possui um denominador a ser expandido, e portanto permanece inalterado no limite HTL. Agora, para dar prosseguimento aos cálculos, analisaremos cada um dos termos acima a fim de identificar quais deles contribuem para uma primeira aproximação, e para isso iremos observar que

$$
\frac{k_{\mu} p_{\nu}}{k \cdot p} \sim \frac{k_{\nu} p_{\mu}}{k \cdot p} \sim 1, \frac{p^{2} p_{\mu} p_{\nu}}{(k \cdot p)^{2}} \sim \frac{p^{2}}{k^{2}} \ll 1, \frac{p^{4}}{(k \cdot p)^{2}} \sim \frac{p^{2}}{k^{2}} \ll 1 .
$$

Obviamente, esses termos muito menores do que 1 são equivalentes à próxima ordem de aproximação, e por isto serão descartados. Desta forma, agruparemos os termos 
provenientes de (4.13a), (4.14a) e (4.14b) para compor $\Pi_{b o s e}$, que representará a parte bosônica da auto-energia dos bósons de calibre.

$$
\begin{aligned}
\prod_{b o s e \mu \nu}^{a b} & =\prod_{G \mu \nu}^{a b}+\prod_{F P \mu \nu}^{a b}+\prod_{T \mu \nu}^{a b} \\
& =\frac{i g^{2} N \delta^{a b}}{2} 2(D-2) \int \frac{d^{D-1} k}{(2 \pi)^{D-1}} \frac{|\vec{k}|^{-1}}{e^{\beta|\vec{k}|}-1}\left[\frac{k_{\mu} p_{\nu}+k_{\nu} p_{\mu}}{k \cdot p}-\frac{p^{2}}{(k \cdot p)^{2}} k_{\mu} k_{\nu}-g_{\mu \nu}\right]
\end{aligned}
$$

Essa parte da auto-energia dos bósons de calibre, possui a mesma estrutura tensorial do termo com loop fermiônico (4.14c), diferindo apenas pela função de distribuição, e por um fator $2(D-2)$ onde para $\Pi_{F \mu \nu}^{a b}$ encontramos $2^{\frac{D}{2}}$. Posto isto, é possível notar que essa forma tensorial em comum, confere a estes termos uma propriedade, que se resume nas seguintes relações:

$$
\begin{gathered}
p^{\mu} \prod_{b o s e \mu \nu}^{a b}=0 \\
p^{\mu} \prod_{F \mu \nu}^{a b}=0 .
\end{gathered}
$$

As duas expressões acima, representam as Identidades de Ward(WARD, 1950), que mesmo antes de impormos o limite HTL, eram respeitadas pela auto-energia total dos bósons de calibre $\Pi=\Pi_{\text {bose }}+\Pi_{F}$. A identidade de Ward resulta da invariância de calibre da ação efetiva térmica.

\subsubsection{Decomposição tensorial das auto-energias}

Nas relações (4.17a) e (4.17b), vemos que $\Pi_{b o s e}$ e $\Pi_{F}$ são ortogonais ao quadrimomento $p$, e portanto podemos decompô-los em termos de dois outros tensores ortogonais à $p^{\mu, \nu}$, e entre si, que serão definidos por

$$
\begin{gathered}
T_{\mu \nu}^{A}=\frac{1}{p^{2}}\left(p_{\mu} p_{\nu}-g_{\mu \nu} p^{2}\right), \\
T_{\mu \nu}^{B}=\bar{u}_{\mu} \bar{u}_{\nu}
\end{gathered}
$$

em que $\bar{u}_{\nu}=u_{\nu}-\frac{p u}{p^{2}} p_{\nu}$, com $u=(1,0,0,0)$ (referencial do banho térmico).

Sem perda de generalidade, podemos imaginar que esse quadrivetor $u$ representa a velocidade de um banho térmico, que é gerado pelo ensemble com temperatura finita. Essa decomposição visa manter o caráter covariante das auto-energias, pois se decidíssemos prosseguir com (4.14c) e (4.16) na forma em que estão, seria necessário efetuar os cálculos componente à componente, assim como é feito, por exemplo, em (KAPUSTA; GALE, 2006). 
Ademais, com o auxílio da quadrivelocidade $u$, é possível escrever as funções de distribuição em um formato explicitamente covariante,

$$
N_{B, F}\left(k_{0}\right)=\frac{1}{e^{\beta k_{0}} \mp 1}=\frac{1}{e^{\beta u \cdot k} \mp 1} .
$$

Antes de prosseguir, devemos notar que a estrutra tensorial em comum entre (4.14c) e (4.16), para a qual efetuaremos a decomposição tensorial é

$$
I_{\mu \nu}=\frac{k_{\mu} p_{\nu}+k_{\nu} p_{\mu}}{k \cdot p}+\frac{p^{2}}{(k \cdot p)^{2}} k_{\mu} k_{\nu}-g_{\mu \nu} .
$$

Além disso, esse tensor pode ser reescrito, genericamente, como $I_{\mu \nu}=A T_{\mu \nu}^{A}+B T_{\mu \nu}^{B}$, que quando contraído ora $\operatorname{com} T_{\mu \nu}^{A}$, e ora $\operatorname{com} T_{\mu \nu}^{B}$, gera o seguinte sistema de equações:

$$
\begin{gathered}
\left\{\begin{array}{l}
T^{A^{\mu \nu}} I_{\mu \nu}=A T^{A^{\mu \nu}} T_{\mu \nu}^{A}+B T^{A^{\mu \nu}} T_{\mu \nu}^{B} \\
T^{B^{\mu \nu}} I_{\mu \nu}=A T^{B^{\mu \nu}} T_{\mu \nu}^{A}+B T^{B^{\mu \nu}} T_{\mu \nu}^{B}
\end{array}\right. \\
\left\{\begin{array}{l}
D-2=A(D-1)+B\left(\frac{(p \cdot u)^{2}-u^{2} p^{2}}{p^{2}}\right) \\
\frac{2(p . u)(k . u)(k \cdot p)-p^{2}(k \cdot u)^{2}-(k \cdot p)^{2} u^{2}}{(k \cdot p)^{2}}=A\left(\frac{(p \cdot u)^{2}-u^{2} p^{2}}{p^{2}}\right)+B\left(\frac{(p \cdot u)^{2}-u^{2} p^{2}}{p^{2}}\right)^{2}
\end{array}\right.
\end{gathered}
$$

Após algumas manipulações algébricas, a solução desse sistema de equações será

$$
\begin{gathered}
A=1-\frac{p^{2}\left[2(k \cdot u)(p \cdot u)(k \cdot p)-p^{2}(k \cdot u)^{2}-(k \cdot p)^{2} u^{2}\right]}{(D-2)(k \cdot p)^{2}\left[(p \cdot u)^{2}-u^{2} p^{2}\right]} ; \\
B=\frac{(D-1)}{(D-2)} \frac{p^{4}\left[2(k \cdot u)(p \cdot u)(k \cdot p)-p^{2}(k \cdot u)^{2}-(k \cdot p)^{2} u^{2}\right]}{(k \cdot p)^{2}\left[(p \cdot u)^{2}-u^{2} p^{2}\right]}-\frac{p^{2}}{(p \cdot u)^{2}-u^{2} p^{2}} .
\end{gathered}
$$

\subsubsection{Integrações}

A decomposição efetuada na subseção anterior, concentrou o caráter tensorial da auto-energia nos tensores apresentados em (4.18), e consecutivamente, fez com que k esteja sempre contraído com algum outro quadrivetor, desta forma, as integrações na parte espacial de $k$ irão agir apenas nos fatores $A$ e $B$. Para que fique mais clara a dependência destes fatores com os momentos internos, os reescreveremos como

$$
\begin{aligned}
A=1 & +\frac{u^{2} p^{2}}{(D-2)\left[(p \cdot u)^{2}-u^{2} p^{2}\right]}-\frac{2 p^{2}(p \cdot u)}{(D-2)\left[(p \cdot u)^{2}-u^{2} p^{2}\right]} \frac{(k \cdot u)}{(k \cdot p)}+ \\
& +\frac{p^{4}}{(D-2)\left[(p \cdot u)^{2}-u^{2} p^{2}\right]} \frac{(k \cdot u)^{2}}{(k \cdot p)^{2}}
\end{aligned}
$$




$$
\begin{aligned}
B= & -\frac{(D-1)}{(D-2)} \frac{p^{4} u^{2}}{\left[(p \cdot u)^{2}-u^{2} p^{2}\right]^{2}}-\frac{p^{2}}{(p \cdot u)^{2}-u^{2} p^{2}}+\frac{(D-1)}{(D-2)} \frac{2(p \cdot u) p^{4}}{\left[(p \cdot u)^{2}-u^{2} p^{2}\right]^{2}} \frac{(k \cdot u)}{(k \cdot p)}+ \\
& -\frac{(D-1)}{(D-2)} \frac{p^{6}}{\left[(p \cdot u)^{2}-u^{2} p^{2}\right]^{2}} \frac{(k \cdot u)^{2}}{(k \cdot p)^{2}} .
\end{aligned}
$$

Embora esses termos contenham muitas componentes, a sua dependência com $k$ é bem simples, pois ela está presente apenas nos fatores $\frac{(k \cdot u)}{(k \cdot p)}$ e $\frac{(k \cdot u)^{2}}{(k \cdot p)^{2}}$, e portanto, necessitaremos efetuar somente as seguintes integrais

$$
\begin{gathered}
I_{1}=\int \frac{d^{D-1 k}}{(2 \pi)^{D-1}} \frac{1}{|\vec{k}|} \frac{1}{e^{\beta|\vec{k}|}-1} ; \\
I_{2}=\int \frac{d^{D-1 k}}{(2 \pi)^{D-1}} \frac{1}{|\vec{k}|} \frac{1}{e^{\beta|\vec{k}|}-1} \frac{1}{p_{0}-|\vec{p}| \cdot \cos (\theta)} ; \\
I_{3}=\int \frac{d^{D-1 k}}{(2 \pi)^{D-1}} \frac{1}{|\vec{k}|} \frac{1}{e^{\beta|\vec{k}|}-1} \frac{1}{\left(p_{0}-|\vec{p}| \cdot \cos (\theta)\right)^{2}} .
\end{gathered}
$$

Nessas integrais escolhemos utilizar o caso bosônico, pois este é mais simples devido a ausência de massa na função de distribuição, porém, a adaptação para o caso fermiônico com massa nula se dará através de apenas um fator, como veremos posteriormente.

Agora, passaremos a utilizar um sistema de coordenadas esféricas nas integrais de (4.26), em que $|\vec{k}|=r$, e o ângulo formado entre a parte espacial dos quadrimomentos $k$ e $p$ será escolhido convenientemente. Como o integrando da primeira dessas integrais não possui dependência angular, iniciaremos por ela,

$$
I_{1}=\frac{\int d \Omega_{D-1}}{(2 \pi)^{D-1}} \int_{0}^{\infty} d r \frac{r^{D-3}}{e^{\beta r}-1} .
$$

As soluções tanto da parte angular, quanto da parte radial desta expressão, já foram introduzidas em (4.5) e (4.6), respectivamente, então

$$
I_{1}=2^{2-D} \frac{T^{D-2} \zeta(D-2) \Gamma(D-2)}{\pi^{\frac{D-1}{2}} \Gamma\left(\frac{D-1}{2}\right)}
$$

Adicionalmente, como todos termos em (4.26) possuem a mesma dependência em relação à parte radial de $\mathrm{k}$, daqui por diante nos concentraremos apenas na parte angular. Posto isto, prosseguiremos com $I_{2}$, cuja parte angular pode ser representada por

$$
\alpha_{2}=\int d \Omega_{D-1} \frac{1}{p_{0}-|\vec{p}| \cdot \cos (\theta)}=\int d \Omega_{D-2} \int d \theta_{D-3} \frac{\sin ^{D-3}\left(\theta_{D-3}\right)}{p_{0}-|\vec{p}| \cdot \cos \left(\theta_{D-3}\right)} .
$$


Aqui vemos, a integral de uma superfície esférica definida em $D-2$ dimensões, cuja solução já foi dada, multiplicando uma integral em $\theta_{D-3}$, na qual faremos a seguinte mudança de variável $v=\cos \left(\theta_{D-3}\right)$.

$$
\alpha_{2}=\frac{2 \pi^{\frac{D-2}{2}}}{\Gamma\left(\frac{D-2}{2}\right)} \int_{-1}^{1} d v \frac{\left(1-v^{2}\right)^{\frac{D-4}{2}}}{p_{0}-|\vec{p}| \cdot v}
$$

Essa integral em $v$ não têm solução simples, e em consulta ao livro Tables of Integral Series and Products(GRADSHTEYN; RYZHIK, 1980), em que definiu-se $B\left(\frac{1}{2}, \frac{D-2}{2}\right)=$ $\Gamma\left(\frac{1}{2}\right) \Gamma\left(\frac{D-2}{2}\right) \Gamma\left(\frac{D-1}{2}\right)$ e $F$ como a função hipergeométrica regularizada ${ }_{2} F_{1}$, concluímos que

$$
\alpha_{2}=\frac{2 \pi^{\frac{D-2}{2}}}{\Gamma\left(\frac{D-2}{2}\right)} \frac{1}{p_{0}-|\vec{p}|} B\left(\frac{1}{2}, \frac{D-2}{2}\right) F\left(\frac{1}{2}, \frac{D-2}{2}, D-2 ; \frac{2|\vec{p}|}{|\vec{p}|-p_{0}}\right)
$$

Compondo esse resultado com a solução da parte radial de (4.26b), temos

$$
I_{2}=2^{2-D} \frac{T^{D-2} \zeta(D-2) \Gamma(D-2)}{\pi^{\frac{D-1}{2}}\left(p_{0}-|\vec{p}|\right)} \Gamma\left(\frac{D-1}{2}\right) F\left(\frac{1}{2}, \frac{D-2}{2}, D-2 ; \frac{2|\vec{p}|}{|\vec{p}|-p_{0}}\right) .
$$

Por fim, nos restou solucionar o termo $I_{3}$, para o qual utilizaremos a equação abaixo, cuja dedução se encontra no apêndice C.

$$
\int d \Omega_{D-1} \frac{p^{2}}{\left(p_{o}-|\vec{p}| \cdot \cos \left(\theta_{D-3}\right)\right)^{2}}=\int d \Omega_{D-1}\left[(D-3)-(D-4) \frac{p_{0}}{p_{o}-|\vec{p}| \cdot \cos \left(\theta_{D-3}\right)}\right]
$$

A partir dela, podemos reescrever $I_{3}$ em relação as outras duas integrais, culminando em

$$
\begin{gathered}
I_{3}=\frac{(D-3)}{p^{2}} I_{1}-\frac{(D-4) p_{0}}{p^{2}} I_{2} \\
I_{3}=2^{2-D} \frac{T^{D-2} \zeta(D-2) \Gamma(D-2)}{\pi^{\frac{D-1}{2}} p^{2}}\left[\frac{(D-3)}{\Gamma\left(\frac{D-1}{2}\right)}-(D-4) \frac{p_{0}}{p_{0}-|\vec{p}|} \Gamma\left(\frac{D-1}{2}\right) \times\right. \\
\left.F\left(\frac{1}{2}, \frac{D-2}{2}, D-2 ; \frac{2|\vec{p}|}{|\vec{p}|-p_{0}}\right)\right] .
\end{gathered}
$$

Assim concluímos o cálculo de todas as componentes de $\Pi_{b o s e}$, sendo que ainda nos resta calcular $\Pi_{F}$. Todavia, a única diferença entre esses dois termos de $\Pi$, é o tipo da distribuição estatística. E como

$$
\int_{0}^{\infty} d r \frac{r^{a}}{e^{\beta r}+1}=\frac{-1+2^{a}}{2^{a}} T^{a+1} \zeta(a+1) \Gamma(a+1),
$$


essa diferença se resumirá a um fator multiplicativo.

Ainda a cerca da expressão acima, é possível notar que nela desprezamos a massa dos férmions, todavia, por estarmos na região da HTL, essa consideração é coerente com todo o desenvolvimento anterior.

Desta forma, podemos somar os resultados para $\Pi_{b o s e}$ e $\Pi_{F}$, e finalmente obter a auto-energia dos bósons de calibre,

$$
\Pi_{\mu \nu}^{a b}=i g^{2} \delta^{a b}\left[N(D-2)+\frac{\left(-8+2^{D}\right)}{2^{\frac{D+2}{2}}} N_{f}\right] \frac{T^{D-2} \zeta(D-2) \Gamma(D-2)}{\pi^{\frac{D-1}{2}} 2^{D-2}} \mathcal{P}_{\mu \nu}(D, p),
$$

em que $\mathcal{P}$ é uma função contendo toda a dependência da auto-energia com os momentos externos, e com a dimensão do espaço-tempo.

Agora, focando apenas neste resultado (4.36), e desconsiderando qualquer problema que possa surgir com a renormalização da teoria de Yang-Mills com temperatura nula, é interessante analisarmos o que ocorre para as diferentes dimensões do espaço-tempo. No caso de $D=2$, isto é, uma dimensão temporal e outra espacial, $\Pi_{\mu \nu}^{a b}$ se torna divergente, assim indicando que o sistema físico clássico advindo do limite de altas temperaturas da teoria de Yang-Mills, definida em uma superfície bi-dimensional, não é factível.

Outro resultado interessante ocorre quando $D=3$, pois neste caso, o termo com loop fermiônico não irá contribuir com (4.36). Já para os casos em que $D \geq 4$, temos $\Pi_{\mu \nu}^{a b}$ finito, fato já esperado, ao menos para $D=4$.

\subsubsection{HTL aplicado à auto-energia dos ghosts}

A expressão da auto-energia dos ghosts é muito mais simples do que a dos bósons de calibre, e após uma soma dos índices de cor em (3.59), temos

$$
\tilde{\Pi}^{a b}=\frac{-i g^{2} N \delta^{a b}}{2} \int \frac{d^{D-1} k}{(2 \pi)^{D-1}} \frac{1}{|\vec{k}|} \frac{p^{2}}{e^{\beta|\vec{k}|}-1}\left[\frac{1}{(k+p)^{2}}+\frac{1}{(k-p)^{2}}\right] .
$$

Agora, ao impormos a condição de hard moments, e na sequência, expandirmos os denominadores no integrando da expressão acima, simplificaremos a auto-energia dos ghosts em

$$
\tilde{\Pi}^{a b}=\frac{i g^{2} N \delta^{a b}}{4} \int \frac{d^{D-1} k}{(2 \pi)^{D-1}} \frac{1}{|\vec{k}|} \frac{1}{e^{\beta|\vec{k}|}-1} \frac{p^{4}}{(k \cdot p)^{2}} .
$$

Analisando o resultado acima, vemos que o fator destacado é claramente muito menor que 1, e portanto, o limite de altas temperaturas da auto-energia dos ghosts será nulo em uma primeira ordem de aproximação. Tal fato, já era esperado, pois como os ghosts representam campos/partículas fictícias geradas por efeitos quânticos, eles não devem possuir um paralelo pertencente à um sistema clássico. 


\subsubsection{HTL aplicado à auto-energia dos férmions}

Para concluir os cálculos deste capítulo, prosseguiremos com a auto-energia dos férmions, e assim, iniciaremos efetuando todas as somas de índices em (3.58) que ainda estão por fazer,

$$
\begin{aligned}
\Sigma_{i j}= & \frac{i g^{2}}{2} \frac{\left(N^{2}-1\right)}{2 N} \delta_{i j} \int \frac{d^{D-1} k}{(2 \pi)^{D-1}}\left[\frac{|\vec{k}|^{-1}}{e^{\beta|\vec{k}|}-1}\left(\frac{(D-2)(\not k+\not p)-D m}{(k+p)^{2}-m^{2}}+k \rightarrow-k\right)+\right. \\
& \left.-\frac{\omega_{k}^{-1}}{e^{\beta \omega_{k}}+1}\left(\frac{(D-2) \not k-D m}{(k-p)^{2}}+k \rightarrow-k\right)\right] .
\end{aligned}
$$

Ao considerarmos o limite de altas temperaturas da expressão acima, ocorrerão algumas diferenças entre os termos bosônicos e fermiônicos de (4.39), onde por bosônicos entendemos ser os termos com $k^{2}=0$, e por fermiônicos os termos com $k^{2}=m^{2}$. Posto isto, iniciaremos com os denominadores bosônicos de $\Sigma_{i j}$, cuja expansão resulta em

$$
\begin{aligned}
\frac{1}{(k \pm p)^{2}-m^{2}} & =\frac{1}{ \pm 2 k \cdot p+p^{2}-m^{2}}=\frac{1}{ \pm 2 k \cdot p} \frac{1}{\left(1 \pm \frac{p^{2}-m^{2}}{2 k \cdot p}\right)} \\
& \approx \frac{1}{ \pm 2 k \cdot p}\left(1 \mp \frac{p^{2}-m^{2}}{2 k \cdot p}\right) .
\end{aligned}
$$

Já para o caso fermiônico, temos

$$
\begin{aligned}
\frac{1}{(k \pm p)^{2}} & =\frac{1}{ \pm 2 k \cdot p+p^{2}+m^{2}}=\frac{1}{ \pm 2 k \cdot p} \frac{1}{\left(1 \pm \frac{\omega_{p}}{2 k \cdot p}\right)} \\
& \approx \frac{1}{ \pm 2 k \cdot p}\left(1 \mp \frac{\omega_{p}}{2 k \cdot p}\right) .
\end{aligned}
$$

Desta forma, a substituição das expansões (4.40) e (4.41) em (4.39), resultará na seguinte expressão para a auto-energia dos férmions:

$$
\begin{aligned}
\Sigma_{i j}= & \frac{i g^{2}}{2} \frac{\left(N^{2}-1\right)}{2 N} \delta_{i j} \int \frac{d^{D-1} k}{(2 \pi)^{D-1}}\left[\frac { | \vec { k } | ^ { - 1 } } { e ^ { \beta | \vec { k } | } - 1 } \left(\frac{(D-2) \not k}{k \cdot p}-\frac{(D-2) \not p\left(p^{2}+m^{2}\right)}{2(k \cdot p)^{2}}+\right.\right. \\
& \left.\left.+\frac{D m\left(p^{2}+m^{2}\right)}{2(k \cdot p)^{2}}\right)+\frac{\omega_{k}^{-1}}{e^{\beta \omega_{k}}+1}\left(\frac{(D-2) \not k}{k \cdot p}-\frac{D m \omega_{p}}{2(k \cdot p)^{2}}\right)\right] .
\end{aligned}
$$

E após selecionarmos os termos que contribuem em uma primeira aproximação, como feito em (4.15), a expressão (4.42) se reduzirá a

$$
\Sigma_{i j}=\frac{i g^{2}}{2} \frac{\left(N^{2}-1\right)}{2 N} \delta_{i j} \int \frac{d^{D-1} k}{(2 \pi)^{D-1}} \frac{1}{|\vec{k}|}\left[\frac{1}{e^{\beta|\vec{k}|}-1} \frac{(D-2) \not k}{k \cdot p}+\frac{1}{e^{\beta|\vec{k}|}+1} \frac{(D-2) \not k}{k \cdot p}\right] .
$$

Ademais, como já não havia mais nenhuma contribuição explícita da massa do férmion em (4.42), e por estarmos em um regime de altas temperaturas, consideramos 
$\omega_{k} \approx|\vec{k}|$ na expressão acima. Além disso, sendo $\not k=\gamma^{0} k_{0}-\gamma^{i} k_{i}$, em que $i$ representa as componentes espaciais do quadrimomento $k$, ao efetuarmos a integração em (4.43) para os valores de $k_{i}$, obteremos integrais nulas, já que estaremos integrando funções ímpares em intervalos simétricos. Portanto, o único termo sobrevivente será

$$
\Sigma_{i j}=\frac{i g^{2}(D-2)}{2} \frac{\left(N^{2}-1\right)}{2 N} \delta_{i j} \gamma^{0} \int \frac{d^{D-1} k}{(2 \pi)^{D-1}}\left[\frac{1}{e^{\beta|\vec{k}|}-1} \frac{1}{k \cdot p}+\frac{1}{e^{\beta|\vec{k}|}+1} \frac{1}{k \cdot p}\right] .
$$

Todavia, integrais destes tipos já foram efetuadas para o caso dos bósons de calibre, então, ao aproveitar esses resultados, obtemos

$$
\begin{array}{r}
\Sigma_{i j}=i g^{2}(D-2) \frac{-1+2^{D-2}}{2^{2 D-4}} \frac{\left(N^{2}-1\right)}{2 N} \delta_{i j} \gamma^{0} \frac{T^{D-2} \zeta(D-2) \Gamma(D-2)}{\pi^{\frac{D-1}{2}}\left(p_{0}-|\vec{p}|\right)} \Gamma\left(\frac{D-1}{2}\right) \times \\
F\left(\frac{1}{2}, \frac{D-2}{2}, D-2 ; \frac{2|\vec{p}|}{|\vec{p}|-p_{0}}\right) .
\end{array}
$$

Assim como ocorreu para o caso dos bósons de calibre, essa auto-energia dos férmions diverge quando estamos em um espaço-tempo bidimensional, em contrapartida, para $D \geq 3$, a função acima é finita. 


\section{Equação de transporte de Boltzmann}

Como alternativa ao método apresentado no capítulo anterior, que utilizou a TQFT seguida do limite de altas temperaturas para calcular as funções de $n$ pontos, iremos agora utilizar um formalismo clássico, que obtém diretamente a expressão das amplitudes térmicas.

Desta forma, iniciaremos a introdução desse outro formalismo através das equações de Wong, sendo que estas descrevem as trajetórias de partículas puntiformes presentes em um plasma de quarks e glúons(WONG, 1970). Adicionalmente, a generalização deste conjunto de equações para um plasma de bósons de calibre e férmions, no escopo de uma teoria de Yang-Mills genérica, é direta, e resulta em

$$
\begin{gathered}
m \frac{d x^{\mu}}{d \tau}=k^{\mu} \\
m \frac{d k^{\mu}}{d \tau}=g Q_{a} F_{a}^{\mu \nu} k_{\nu} \\
m \frac{d Q^{a}}{d \tau}=-g f^{a b c} k^{\mu} A_{b \mu} Q_{c}
\end{gathered}
$$

Essas equações, juntamente com $D_{a b \nu} F_{b}^{\mu \nu}=J_{a}^{\nu}$, em que $J_{a}$ é a corrente associada às cargas $Q_{a}$, formam um conjunto auto-consistente de equações de Vlasov não-abelianas. Embora essas relações sejam definidas para partículas puntiformes, elas serão utilizadas neste capítulo para descrever excitações coletivas no plasma de Yang-Mills.

No capítulo anterior, quando utilizamos o limite de altas temperaturas, os momentos internos aos loops eram da mesma ordem da temperatura, e adicionalmente, estes eram muito maiores do que os momentos externos. Em paralelo, para o nosso plasma clássico, consideraremos que suas partículas irão interagir apenas com as excitações coletivas do plasma, ignorando assim interações pontuais. De forma quantitativa, isto equivale a considerar a distância média entre as partículas da ordem de $1 / T$, e o comprimento característicos dessas excitações da ordem de $1 /(g T)$.

Aliás, ao correlacionarmos os momentos internos aos loops com a distância média entre as partículas, e os momentos externos com os comprimentos de onda das excitações do plasma, vemos que os dois limites discutidos no parágrafo precedente são equivalentes. Sendo assim, os dois formalismos, quântico (em altas temperaturas) e clássico, são duas formas diferentes de observar o mesmo problema físico, e como veremos, produzem um mesmo resultado.

Além disso, no artigo Semi-classical transport theory for non-abelian plasmas(LITIM; MANUEL, 2002), foi demonstrado, para estados quânticos bem localizados, que as variáveis 
de movimento referentes à um pacote de onda (quadrimomento mecânico $p$, valor esperado para o centro de energia $x$, e valor esperado para a carga $Q$ ) também obedecem as equações de Wong, (5.1). Portanto, para o plasma clássico em questão, ao invés de considerarmos as variáveis que descrevem o movimento de uma única partícula, utilizaremos aquelas atreladas à um pacote de onda. Sendo assim, quem irá se comportar classicamente serão as excitações presentes no plasma, e não suas partículas isoladamente.

Associado à esse plasma clássico, descrito nos parágrafos anteriores, existe uma função de distribuição, que de forma geral, obedece à equação de Boltzmann,

$$
\frac{d}{d \tau} f(x, k, Q)=C[f]
$$

em que $\tau$ é o tempo próprio, e $C$ é o termo de colisão, que assumiremos depender da função de distribuição.

Como o limite que estamos utilizando considera apenas as interações entre partículas e campos médios, podemos desprezar o termo de colisão. Desta forma, expandindo a derivação total em relação ao tempo próprio, temos

$$
\frac{d}{d \tau} f(x, k, Q)=\frac{d x^{\mu}}{d \tau} \frac{\partial}{\partial x^{\mu}} f(x, k, Q)+\frac{d k^{\mu}}{d \tau} \frac{\partial}{\partial k^{\mu}} f(x, k, Q)+\frac{d Q^{a}}{d \tau} \frac{\partial}{\partial Q_{a}} f(x, k, Q)=0
$$

Agora, substituindo na equação acima, as derivadas parcias de $p, x$ e $Q$ em relação ao tempo próprio contidas em (5.1), obtemos

$$
k^{\mu}\left(\frac{\partial}{\partial x^{\mu}}-g Q_{a} F_{a \mu \nu} \frac{\partial}{\partial k_{\nu}}-g f^{a b c} A_{b \mu} Q_{c} \frac{\partial}{\partial Q_{a}}\right) f(x, k, Q)=0 .
$$

A priori, essa função de distribuição engloba todos os regimes físicos deste sistema, e por isto, é necessário restringi-la ao caso em que as excitações serão provenientes de pequenas pertubações do estado em equilíbrio. Posto isto, faremos uma expansão da função distribuição em potências da constante de acoplamento,

$$
f(x, x, Q)=f^{(0)}\left(k_{0}\right)+f^{(1)}(x, k, Q)+f^{(2)}(x, k, Q)+\ldots
$$

Como essa expansão é no entorno de um estado em equilíbrio, a função $f^{(0)}\left(k_{0}\right)$ representará justamente este estado, e por isto depende apenas da energia e estatística das partículas. Substituindo a expressão acima em (5.4), obtemos

$$
k^{\mu}\left(\frac{\partial}{\partial x^{\mu}}-g f^{a b c} A_{b \mu} Q_{c} \frac{\partial}{\partial Q_{a}}\right) f^{(1)}(x, k, Q)=g Q_{a} k^{\mu} F_{a \mu \nu} \frac{\partial}{\partial k_{\nu}} f^{(0)}\left(k_{0}\right),
$$

para a primeira ordem em $g$.

As próximas ordens na constante de acoplamento permitem correlacionar $f^{(n+1)}$ com $f^{(n)}$, e consecutivamente, calcular os termos seguintes de (5.5). Portanto, este método 
possibilita calcular qualquer que seja a ordem de aproximação, embora a dificuldade de cálculo aumente substancialmente com a potência de $g$, como será visto no capítulo seguinte.

Ademais, com auxílio destas aproximações da função de distribuição, torna-se possível calcular algumas quantidades físicas, como por exemplo, a densidade de corrente, que pode ser escrita como

$$
j_{a}^{\mu}(x, k)=g \int d Q p^{\mu} Q_{a} f(x, k, Q),
$$

e esta por sua vez correlaciona-se com corrente total através de

$$
J_{a}^{\mu}(x)=\sum_{\text {partículas }, \text { spins }} \int d k j_{a}^{\mu}(x, k)
$$

em que a somatória inclui todos os tipos de partículas e graus de liberdade dos spins.

Desta forma, a fim de extrair a densidade de corrente da equação de Boltzmann, iremos multiplicar (5.6) por $g k^{\alpha} Q_{d}$ e efetuar uma integração no espaço das cargas,

$g \int d Q k^{\alpha} Q_{d} k^{\mu}\left(\frac{\partial}{\partial x^{\mu}}-g f^{a b c} A_{b \mu} Q_{c} \frac{\partial}{\partial Q_{a}}\right) f^{(1)}(x, k, Q)=g \int d Q k^{\alpha} Q_{d} Q_{a} k^{\mu} F_{a \mu \nu} \frac{\partial}{\partial k_{\nu}} f^{(0)}\left(k_{0}\right)$.

No primeiro termo do lado esquerdo desta equação, temos uma derivada espacial, que comuta com todos os termos a sua esquerda, então

$$
g \int d Q k^{\alpha} Q_{d} k^{\mu} \frac{\partial}{\partial x^{\mu}} f^{(1)}(x, k, Q)=\frac{\partial}{\partial x^{\mu}} j_{d}^{(1)^{\alpha}}(x, k) .
$$

Já para o segundo termo do lado esquerdo de (5.9), iremos efetuar uma integração por partes em $Q$, que irá gerar um termo de superfície, cujo valor é nulo, pois estamos supondo que todos os campos se anulam a uma certa distância do centro da pertubação, e assim obteremos

$$
g \int d Q k^{\alpha} Q_{d} k^{\mu}(-g) f^{a b c} A_{b_{\mu}} Q_{c} \frac{\partial}{\partial Q_{a}} f^{(1)}(x, k, Q)=g f^{d b c} k^{\mu} A_{b \mu} j_{d}^{(1)^{\alpha}}(x, k) .
$$

Por fim, o lado direito de (5.9) culmina em

$$
g^{2} \int d Q k^{\alpha} Q_{d} Q_{a} k^{\mu} F_{a \mu \nu} \frac{\partial}{\partial k_{\nu}} f^{(0)}\left(k_{0}\right)=g^{2} k^{\alpha} k^{\mu} F_{d \mu \nu} \frac{\partial}{\partial k_{v}} \int d Q Q_{d} Q_{a} f^{(0)}\left(k_{0}\right) .
$$

Aqui vemos, que essa integral restante no espaço das cargas irá depender da espécie das partículas, isto é, se elas são bósons ou férmions, sendo assim, por ora definiremos:

$$
\mathcal{F}_{a d}\left(k_{0}\right)=\int d Q Q_{d} Q_{a} f^{(0)}\left(k_{0}\right)
$$


Agora, retornando (5.10-5.12) em (5.9), temos

$$
k_{\mu} D_{a b}{ }^{\mu} j_{b}^{(1)}{ }_{b}^{\alpha}(x, k)=g^{2} k^{\alpha} k^{\mu} F_{b \mu \nu} \frac{\partial}{\partial k_{\nu}} \mathcal{F}_{b a}\left(k_{0}\right) .
$$

Aqui culminamos com uma primeira correção para a densidade de corrente em função do estado de equilíbrio, portanto, (5.14) traduz os efeitos mais significativos das pertubações em $f^{(0)}\left(k_{0}\right)$. Ademais, se introduzirmos em (5.14), uma integração no espaço dos momentos $k$, e uma soma sob todas as espécies de partículas e graus de liberdade dos spins, obteremos uma expressão para a corrente total,

$$
J_{b}^{(1) \mu}(x)=g^{2} \int d k k^{\alpha} k^{\mu}\left(\frac{1}{k \cdot D}\right)_{a c} F_{c \mu \nu} \frac{\partial}{\partial k_{\nu}} \sum_{\text {partículas }, \text { spins }} \mathcal{F}_{b a}\left(k_{0}\right) .
$$

Redefinindo $\tilde{\mathcal{F}}_{b a}\left(k_{0}\right)=\sum_{\text {partículas,spins }} \mathcal{F}_{b a}\left(k_{0}\right)$ neste resultado, e efetuando uma integração por partes em $k$, concluímos que

$$
J_{b}^{(1)}{ }_{b}^{\mu}(x)=-g^{2} \int d k \frac{\left(k . D_{a c} k^{\mu} g^{\alpha \nu}-k^{\alpha} k^{\mu} D_{a c}^{\nu}\right)}{(k . D)^{2}} F_{c \mu \nu} \tilde{\mathcal{F}}_{b a}\left(k_{0}\right) .
$$

Nesta passagem, consideramos novamente que o termo de superfície se anula nos extremos, e utilizamos a antissimetria do tensor $F$.

No apêndice A, defini-se uma relação entre a corrente e a ação efetiva, (A.14), que aqui suporemos ser válida para a corrente em (5.16), resultando em

$$
\Gamma_{\text {transp }}=\int d^{D} x A_{a \mu}(x) J_{a}^{\mu}(x)
$$

Essa relação será a base da equivalência entre o formalismo que estamos desenvolvendo neste capítulo, e o limite de altas temperaturas da TQFT. Posto isto, substituiremos (5.16) em (5.17), resultando em

$$
\begin{aligned}
\Gamma_{\text {transp }}^{(1)} & =\int d^{D} x A_{a \mu}(x) J_{a}^{(1)}{ }_{a}^{\mu}(x) \\
& =-g^{2} \int d^{D} x A_{a \mu} \int d k \frac{\left(k \cdot D_{b c} k^{\mu} g^{\alpha \nu}-k^{\alpha} k^{\mu} D_{b c}^{\nu}\right)}{(k \cdot D)^{2}} F_{c \mu \nu} \tilde{\mathcal{F}}_{a b}\left(k_{0}\right) \\
& =g^{2} \int d^{D} x \int d k \frac{\left(k \cdot D_{b c} A_{a}^{\nu}-k^{\alpha} D_{b c}^{\nu} A_{a \alpha}\right)}{(k \cdot D)^{2}} k^{\mu} F_{c \mu \nu} \tilde{\mathcal{F}}_{a b}\left(k_{0}\right) \\
& =g^{2} \int d^{D} x \int d k F_{b \alpha} \frac{k^{\alpha} k^{\mu}}{(k \cdot D)^{2}} F_{a \mu \nu} \tilde{\mathcal{F}}_{a b}\left(k_{0}\right) .
\end{aligned}
$$

$\mathrm{Na}$ passagem de (5.18b) para (5.18c), efetuamos uma integração por partes no espaço das configurações, e em seguida, após reorganizamos alguns termos e renomearmos certos índices mudos, culminamos com (5.18d).

Esse último resultado é explicitamente invariante via transformações de calibre, e sua forma se repete para várias outras teorias, incluindo a QED e Yang-Mills supersimétricas(CZAJKA; MRICZYSKI, 2015). Isto demonstra, que embora essas teorias de calibre 
sejam microscopicamente muito diferentes, elas possuem um mesmo comportamento para longos comprimentos de onda. Podemos entender melhor esse fenômeno, se o compararmos ao problema das antenas no eletromagnetismo, pois de forma geral, a geometria de uma antena governa as características de suas emissões em uma região de curto alcance, porém, a longas distâncias, toda onda emitida por uma antena se comporta como fosse esférica.

Agora, ao invés de prosseguir com (5.18d), como faremos no próximo capítulo, iremos aproveitar para introduzir a conexão entre corrente e as funções de $n$ pontos, (A.24), que para o caso da auto-energia se escreve como

$$
\Pi_{a b}^{\mu \nu}(x, y)=\left.\frac{\delta J_{a}^{(1)^{\mu}}(x)}{\delta A_{\nu}^{b}(y)}\right|_{A=0}
$$

A relação acima extrai a função de dois pontos, ou auto-energia, da expressão para a corrente, e pode ser generalizada para qualquer número de pontos. Por exemplo, para a função de três pontos temos

$$
g \Gamma_{a b c}^{\mu \nu \lambda}(x, y, z)=\left.\frac{\delta^{2}\left[{J^{(1)}}_{a}^{\mu}(x)+J_{a}^{(2)^{\mu}}(x)\right]}{\delta A_{\nu}^{b}(y) \delta A_{\lambda}^{c}(z)}\right|_{A=0} .
$$

Como é notável acima, foi necessário incluir no cálculo da função de 3 pontos a primeira e a segunda ordem de aproximação da corrente. Isto ocorreu, pois $J^{(1)}$ possui termos proporcionais à $A^{2}, A^{3}$ e $A^{4}$, enquanto que $J^{(2)}$ contém termos proporcionais à $A^{2}, A^{3}, A^{4}$ e $A^{5}$. Portanto, para uma função de n pontos, será necessário obter todas as ordens da corrente até $J^{(n-1)}$.

Retornando ao cálculo da auto-energia, substituiremos (5.15) em (5.19), para obter

$$
\begin{aligned}
\Pi_{a b}^{\mu \nu}(x, y) & =\left.g^{2} \int d k k^{\alpha} k^{\mu} \frac{\delta}{\delta A_{\nu}^{b}(y)}\left[\left(\frac{1}{k . D}\right)_{c d} F_{d \mu \nu}\right] \frac{\partial}{\partial k_{\nu}} \tilde{\mathcal{F}}_{c a}\left(k_{0}\right)\right|_{A=0} \\
& =g^{2} \int d k k^{\mu} \frac{1}{k . \partial}\left[g_{\beta}^{\nu} p . \partial\left(\delta^{(n)}(x-y)\right)-k^{\nu} \partial_{\beta}\left(\delta^{(n)}(x-y)\right)\right] \frac{\partial}{\partial k_{\beta}} \tilde{\mathcal{F}}_{a b}\left(k_{0}\right),
\end{aligned}
$$

na qual fica explícito que a função de dois pontos depende apenas da distância entre $x$ e $y$. Assim, ao efetuarmos uma integração por partes no espaço dos momentos nesta equação acima, temos

$$
\Pi_{a b}^{\mu \nu}(x, y)=g^{2} \int d k\left[g^{\mu \nu}-\frac{k^{\nu} \partial^{\mu}+k^{\mu} \partial^{\nu}}{k . \partial}+\frac{k^{\mu} k^{\nu} \partial^{2}}{(k . \partial)^{2}}\right]\left(\delta^{(n)}(x-y)\right) \tilde{\mathcal{F}}_{a b}\left(k_{0}\right) .
$$

Como é usual expressar as funções de n pontos em relação aos momentos, iremos efetuar uma transformada de Fourier nesta expressão,

$$
\Pi_{a b}^{\mu \nu}(p)=g^{2} \int d k\left[g^{\mu \nu}-\frac{k^{\nu} p^{\mu}+k^{\mu} p^{\nu}}{k \cdot p}+\frac{k^{\mu} k^{\nu} p^{2}}{(k \cdot p)^{2}}\right] \tilde{\mathcal{F}}_{a b}\left(k_{0}\right) .
$$


Nessa atual forma, já é possível notar uma grande semelhança entre esta expressão da auto-energia, e a obtida via HTL, (4.16), sendo que a estrutura tensorial é exatamente a mesma. Portanto, para completarmos a comparação, ainda é necessário efetuar a integral no espaço dos momentos, e a no espaço das cargas, contidas em $\tilde{\mathcal{F}}$.

\subsection{Espaço das cargas}

Antes de prosseguirmos com a integração dos momentos em (5.23), efetuaremos a integral em $Q$, cuja medida de integração para $S U(3)$ é

$$
d Q=d^{8} Q C_{r} \delta\left(Q_{a} Q_{a}-q_{2}\right) \delta\left(d_{a b c} Q_{a} Q_{b} Q_{c}-q_{3}\right)
$$

em que $d_{a b c}$ são as constantes totalmente simétricas associadas à $S U(3)$, e $C_{r}$ é a constante de normalização da medida de integração definida por $\int d Q=1$.

As duas funções delta em (5.24), visam garantir a invariância das constantes de Casimir $q_{2}$ e $q_{3}$, que são específicas do grupo $S U(3)$. Aqui especificamos $N=3$, pois infelizmente não é possível definir uma medida de integração genérica para $S U(N)$ de forma compacta, mas independentemente de qual seja o valor de $N$, sempre podemos impor que todas as constantes de Casimir serão conservadas, e assim garantimos que apenas estados físicos reais estarão contribuindo na expressão de $\tilde{\mathcal{F}}$. Porém, mesmo com essa dificuldade, ainda é possível continuar os cálculos para $S U(N)$, pois a definição da constante de Casimir quadrática, $C_{2}$, é válida para qualquer que seja $N$.

$$
\int d Q Q_{a} Q_{b}=C_{2} \delta_{a b}
$$

De forma geral, essa constante depende da dimensão e representação do grupo à qual as partículas pertencem, por exemplo, para aquelas cuja representação for a adjunta do grupo $S U(N)$, que é o caso dos bósons de calibre, temos $C_{2}=N$; já para as partículas na representação fundamental, o que ocorre para os férmions, $C_{2}$ será igual a $1 / 2$. Como resultado direto da definição acima, temos

$$
\begin{aligned}
\mathcal{F}_{a b}\left(k_{0}\right) & =f^{(0)}\left(k_{0}\right) \int d^{8} Q \delta\left(Q_{a} Q_{a}-q_{2}\right) \delta\left(d_{a b c} Q_{a} Q_{b} Q_{c}-q_{3}\right) Q_{b} Q_{a} \\
& =C_{2} \delta_{a b} f^{(0)}\left(k_{0}\right)
\end{aligned}
$$

Aqui vemos o fator $f^{(0)}\left(k_{0}\right)$, que como já foi dito, é igual a função de distribuição de um sistema em equilíbrio, portanto, quando as partículas forem bósons teremos $f^{(0)}\left(k_{0}\right)=$ $N_{B}\left(k_{0}\right)$, e em contrapartida, quando elas forem férmions, temos $f^{(0)}\left(k_{0}\right)=N_{F}\left(k_{0}\right)$. 


\subsection{Integração no espaço dos momentos}

Além da conservação das constantes de Casimir, imposta em (5.24), para que o sistema contenha apenas estados físicos reais, ainda devemos impor em nossos cálculos a positividade da energia, e que as partículas estejam na camada de massa. Assim, definiremos a medida de integração nos momentos, como

$$
d k=\frac{d^{D} k}{(2 \pi)^{D-1}} 2 \theta\left(k_{0}\right) \delta\left(k^{2}-m^{2}\right)
$$

Ao invés de incluirmos essa função degrau $\theta$, e a função delta na medida de integração, poderíamos ter definido a função $f^{(0)}$ de forma a contemplar essas condições, e assim o faremos no próximo capítulo.

Ao substituir essa medida de integração, (5.27), e o valor final de $\tilde{\mathcal{F}}$, (5.26), em (5.23), obtemos

$$
\begin{aligned}
& \Pi_{a b}^{\mu \nu}=g^{2} \sum_{\text {partículas, spins }} C_{2} \delta_{a b} \int \frac{d^{D-1} k}{(2 \pi)^{D-1}} \int d k_{0} 2 \theta\left(k_{0}\right) \delta\left(k^{2}-m^{2}\right) N_{B, F}\left(k_{0}\right) \times \\
& {\left[g^{\mu \nu}-\frac{k^{\nu} p^{\mu}+k^{\mu} p^{\nu}}{k \cdot p}+\frac{k^{\mu} k^{\nu} p^{2}}{(k \cdot p)^{2}}\right] . }
\end{aligned}
$$

Agora, a fim de efetuar a integral em $k_{0}$, devemos reescrever a função delta da expressão acima na seguinte forma:

$$
\delta\left(k^{2}-m^{2}\right)=\delta\left(k_{0}^{2}-\vec{k}^{2}-m^{2}\right)=\delta\left(k_{0}^{2}-\omega_{k}^{2}\right)=\frac{1}{2 \omega_{k}}\left[\delta\left(k_{0}+\omega_{k}\right)+\delta\left(k_{0}-\omega_{k}\right)\right] .
$$

E assim, ao integrar (5.28) em $k_{0}$, a função $\theta\left(k_{0}\right)$ irá garantir que apenas $\delta\left(k_{0}-\omega_{k}\right)$ contribua, portanto,

$$
\Pi_{a b}^{\mu \nu}=g^{2} \sum_{\text {partículas,spins }} C_{2} \delta_{a b} \int \frac{d^{D-1} k}{(2 \pi)^{D-1}} \frac{N_{B, F}\left(k_{0}\right)}{k_{0}}\left[g^{\mu \nu}-\frac{k^{\nu} p^{\mu}+k^{\mu} p^{\nu}}{k \cdot p}+\frac{k^{\mu} k^{\nu} p^{2}}{(k \cdot p)^{2}}\right] .
$$

Por fim, nos resta apenas a somatória acima, onde a contabilização das espécies de partículas produzirá um fator $2 N_{f}$ para os férmions, pois devemos considerar as partículas e antipartículas. Já para o fator, $f_{S}$, proveniente da soma dos graus de liberdade do spin, utilizaremos o resultado do artigo Systematics of higher-spin gauge fields(WIT; FREEDMAN, 1980), que define

$$
f_{S}=\left\{\begin{array}{l}
2\left(\begin{array}{c}
S+D-5 \\
S-1
\end{array}\right)+\left(\begin{array}{c}
S+D-5 \\
S
\end{array}\right), \text { para bósons com spin igual a } S \\
2^{\frac{D}{2}\left(\begin{array}{c}
S+D-4 \\
S
\end{array}\right), \text { para férmions com spin igual a } S+\frac{1}{2} .}
\end{array}\right.
$$

Essa expressão acima é válida apenas para $D \geq 4$, e resulta em $D-2$ para bósons com spin igual a 1 . Em contrapartida, $f_{S}=2^{\frac{D}{2}}$ para os férmions com spin igual a 1/2. 
Assim, reunindo todos esses resultados, vemos que o valor encontrado para a autoenergia via equação de transporte é exatamente igual ao obtido via HTL em (4.16), a menos de um fator $-i$. Todavia, este irá ocorrer naturalmente após efetuarmos uma continuação analítica em (4.16), então o resultado obtido pelos dois formalismos é exatamente igual.

Portanto, concluímos em uma primeira ordem de aproximação, que o formalismo obtido através do limite de altas temperaturas da TQFT é equivalente ao obtido via equação de transporte de Boltzmann, e consecutivamente, vimos que ao aplicarmos as condições previstas pelo HTL na TQFT iremos obter seu limite clássico. 


\section{Limite de altas temperaturas da QED e a equação de transporte}

Verificamos no capítulo 5 que o limite de altas temperaturas da auto-energia, em uma teoria não abeliana, pode ser equivalentemente obtido usando o formalismo de equação de transporte. Sabemos que esse resultado pode ser generalizado para todas as ordens. Ou seja, todas as funções de $n$ glúons, no limite de altas temperaturas, podem ser obtidas via equação de transporte (KELLY et al., 1994) (esse resultado foi também generalizado para o espaço-tempo curvo (BRANDT; FRENKEL; TAYLOR, 1995b)). Nosso principal objetivo, no presente capítulo, será investigar se o mesmo ocorre em uma teoria abeliana, mesmo considerando que cada uma das funções de $n$ fótons possui uma dependência distinta na temperatura. Note que essa questão não foi considerada anteriormente, simplesmente porque a contribuição dominante da QED é de fato aquela advinda da função de dois fótons (auto-energia). Mas, em princípio, as outras funções de Green podem (ou não) ter uma correspondente descrição via equação de Boltzmann (BRANDT; FERREIRA; THUORST, 2015).

Na QED, a dependência com a temperatura varia de acordo com a ordem de aproximação, ou em termos de gráficos de Feynman, com a quantidade de pernas externas. Em contraste, o mesmo não ocorre para as teorias não-abelianas, já que todos os seus termos dependem igualmente da temperatura, como por exemplo, para a QCD, que possui todas amplitudes térmicas proporcionais à $T^{2}$ no limite de massa nula, em $D=4$ (FRENKEL; TAYLOR, 1990).

Além desta propriedade, podemos também considerar uma situação mais geral, tal que as espécies de cargas distintas são tratadas separadamente. Para tornar esta distinção possível, consideraremos um plasma com uma distribuição de cargas genérica e não neutra, assim evitaremos alguns cancelamentos, pois se utilizássemos um plasma neutro, todas as contribuições de gráficos de Feynman com um número ímpar de vértices (férmion-fóton) se anulariam.

Dentre as amplitudes térmicas existentes, utilizaremos as provenientes de gráficos de Feynman como o exposto na figura 15, que formam diagramas 1PI das amplitudes com $n$ fótons.

Ao comparar todas as contribuições destas amplitudes térmicas, verificaremos, no caso de um plasma neutro, que o termo dominante na ação efetiva da QED é gerado pelo tensor de polarização do fóton(DESER; GRIGUOLO; SEMINARA, 1998), cuja dependência térmica possui um fator $T^{2}$. Já as contribuições individuais de pósitrons ou 


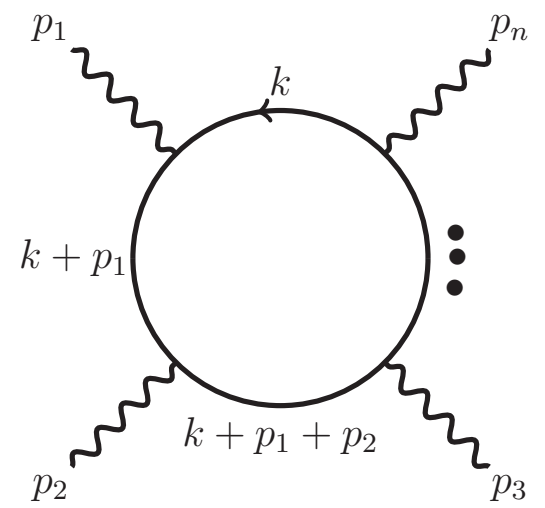

Figura 15 - Diagrama de um loop fermiônico com $n$ fótons externos.

elétrons geram um termo dominante proporcional a $T^{3}$, proveniente da função de um fóton $(n=1)$. Os outros termos ou são proporcionais à uma menor potência da temperatura ou possuem combinações de potências e logaritmos da temperatura. Para o cálculo destas amplitudes via TQFT utilizaremos o formalismo do tempo imaginário conforme o capítulo 2, e após aplicaremos o limite de altas temperaturas.

Iniciaremos pelos cálculos via equação de transporte de Boltzmann, que, como será visto na próxima seção, leva a uma ação efetiva explicitamente invariante por transformações de calibre, para qualquer que seja $n$, pois esta será expressa em termos de tensores eletromagnéticos. Em contrapartida, não é possível obter um resultado, com esta mesma característica explícita, via um procedimento baseado em primeiros princípios, que é o caso da QED com temperatura finita.

Já na terceira seção, calcularemos as mesmas quantidades físicas, para a QED em altas temperaturas, o que nos permitirá comparar os dois formalismos. Este procedimento se restringe até 4 fótons externos, pois mesmo utilizando métodos computacionais (HSIEH; YEHUDAI, 1992), a quantidade de cálculos a serem efetuados cresce consideravelmente com a ordem de aproximação.

Por fim, discutiremos a dependência térmica das amplitudes encontradas. Ademais, o caso de altíssimas temperaturas, ou equivalentemente, o caso no qual a massa é desprezível, envolve termos com divergências infravermelhas, e será tratado a parte no apêndice D.

\subsection{Equação de Boltzmann}

Uma partícula pertencente a um plasma de elétrons ou pósitrons, com quadrimomento $k$ e quadrivetor de posição $x$, e sob a ação de um campo eletromagnético externo $F^{\mu \nu}$, tem sua trajetória descrita pelas seguintes equações de movimento:

$$
m \frac{d x^{\mu}}{d \tau}=k^{\mu}
$$




$$
m \frac{d k^{\mu}}{d \tau}=-q F^{\mu \nu} k_{\nu}
$$

em que $m, \tau$ e $q$, são respectivamente, a massa, o tempo próprio e a carga elétrica da partícula, que assume valor igual a $+e$ para o pósitron e $-e$ para o elétron. Essas duas expressões são claramente o limite abeliano das equações de Wong contidas em (5.1). Além disso, como se trata do caso abeliano de uma teoria de calibre, o tensor eletromagnético se resumirá a $F_{\mu \nu}=\partial_{\mu} A_{\nu}-\partial_{\nu} A_{\mu}$.

Nesta seção, assim como ocorreu no Capítulo 5, ignoraremos os efeitos das colisões entre as partículas, portanto, consideraremos apenas as interações dos elétrons e pósitrons com os campos eletromagnéticos externos.

Como agora o nosso espaço de fase é composto apenas pelas posições e pelos momentos, ao substituir as derivadas dos quadrivetores $x$ e $k$ em relação ao tempo próprio em (5.3), obteremos uma outra equação de transporte mais simples,

$$
k^{\mu} \frac{\partial}{\partial x^{\mu}} f(x, k, q)=q F_{\mu \nu} k^{\nu} \frac{\partial}{\partial k_{\mu}} f(x, k, q) .
$$

Equivalentemente ao feito em (5.5), podemos expandir a função de distribuição em potências dos campos eletromagnéticos, resultando em uma solução da equação de transporte,

$$
f^{(n)}(x, k, q)=\frac{q}{k \cdot \partial} F_{\mu \nu} k^{\nu} \frac{\partial}{\partial k_{\mu}} f^{(n-1)}(x, k, q) .
$$

Essa equação possui um caráter iterativo, o que permite visualizar a forma de todos os termos componentes da função de distribuição. Ademais, a presença de $F_{\mu \nu}$ na expressão acima, deixa claro que as interações são resultado da presença de campos externos.

Novamente, teremos uma corrente, cuja expressão é

$$
{ }^{ \pm} J_{\mu}(x)= \pm e C \int d^{4} k k_{\mu} f(x, k, \pm e),
$$

em que $C$ representa os graus de liberdade do spin.

Esta corrente, obviamente, depende do sinal da carga, ou equivalentemente, se a partícula é um elétron ou um pósitron, e por isto seguiremos fazendo essa distinção, pois se tivéssemos tratando de um plasma neutro teríamos uma corrente total igual a:

$$
J_{\text {total }}={ }^{+} J+{ }^{-} J
$$

Antes de continuarmos, será conveniente analisarmos o que ocorre ao invertermos os sinais dos momentos internos. Portanto, aplicando $k \rightarrow-k$ na equação de transporte, temos

$$
k^{\mu} \frac{\partial}{\partial x^{\mu}} f(x,-k, q)=-q F_{\mu \nu} k^{\nu} \frac{\partial}{\partial k_{\mu}} f(x,-k, q) .
$$


Em contrapartida, a conjugação de carga $(q \rightarrow-q)$, resulta em

$$
k^{\mu} \frac{\partial}{\partial x^{\mu}} f(x, k,-q)=-q F_{\mu \nu} k^{\nu} \frac{\partial}{\partial k_{\mu}} f(x, k,-q) .
$$

Assim, ao compararmos (6.6) com (6.7), concluímos que $f(x, k,-e)=f(x,-k, e)$. Posto isto, demonstramos a equivalência da conjugação de carga com a inversão de sinal do momento interno para este plasma de elétrons e pósitrons.

Utilizando esta equivalência, e expandindo a expressão iterativa da equação de transporte até relacionarmos $f^{(n)}$ com $f^{(0)}$, obtém-se

$$
f^{(n)}(x, k, q)=(\mp e)^{n}\left(\frac{1}{k \cdot \partial} F_{\mu \nu} k^{\nu} \frac{\partial}{\partial k_{\mu}}\right)^{n} f^{(0)}\left(k_{0}\right),
$$

que quando substituída na expressão da corrente para um plasma neutro, deixa claro que os termos com $n$ ímpares serão nulos.

Como já foi citado no capítulo anterior, uma das formas de conectarmos a teoria clássica com a TQFT, é via o cálculo da ação efetiva, e para isto, substituiremos as correntes ${ }^{ \pm} J_{\mu}$ em (5.17).

$$
{ }^{ \pm} \Gamma_{\text {transp }}=\int d^{4} x^{ \pm} J_{\mu}(A) A^{\mu}(x)
$$

Adicionalmente, para o caso do nosso plasma de elétrons e/ou pósitrons em um regime semi-clássico, a função de distribuição pode ser escrita como

$$
f(x, k, e)=\frac{1}{(2 \pi)^{3}} 2 \theta\left(k_{0}\right) \delta\left(k^{2}-m^{2}\right) F(x, k, e) .
$$

Note que agora, estamos garantindo a positividade da energia e a condição da camada de massa diretamente via função de distribuição, em oposição ao feito em (5.27).

A função $F(x, k, e)$ depende dos campos externos, podendo ser em geral bastante complicada. Como nosso objetivo é fazer um tratamento perturbativo, é importante considerar primeiramente a situação em que os campos externos são nulos (ordem zero). Dada essa condição, a função $F^{0}\left(k_{0}\right)$ dependerá apenas das energias das partículas e da estatística. No presente caso, teremos a distribuição de equilíbrio dada por

$$
F^{(0)}\left(k_{0}\right)=N_{F}\left(k_{0}\right)=\frac{1}{e^{\frac{k_{0}}{T}}+1} .
$$

Como qualquer função independente de $x$ será solução da equação de transporte em equilíbrio térmico, essa distribuição de Fermi-Dirac configura um caso especial, e sua utilização irá gerar a seguinte corrente:

$$
{ }^{ \pm} J_{\mu}^{(0)}= \pm \frac{e C}{(2 \pi)^{3}} \int d^{4} k 2 \theta\left(k_{0}\right) \delta\left(k^{2}-m^{2}\right) N_{F}\left(k_{0}\right) k_{\mu} .
$$


A substituição de (5.29) nesta equação, permitirá a integração em $k_{0}$ imediatamente, resultando em uma expressão, cuja função de distribuição depende apenas da parte radial do momento interno.

$$
{ }^{ \pm} J_{\mu}^{(0)}= \pm \frac{e C}{(2 \pi)^{3}} \int \frac{d^{3} k}{\sqrt{\vec{k}^{2}+m^{2}}} N_{F}\left(\sqrt{\vec{k}^{2}+m^{2}}\right) k_{\mu}
$$

Agora, basta introduzir essa corrente em (6.9) para obter a primeira ordem de aproximação da ação efetiva:

$$
{ }^{ \pm} \Gamma_{\text {transp }}^{(1)}= \pm \frac{e C}{(2 \pi)^{3}} \int d^{4} x \int \frac{d^{3} k}{\sqrt{\vec{k}^{2}+m^{2}}} N_{F}\left(\sqrt{\vec{k}^{2}+m^{2}}\right) k_{\mu} A^{\mu}(x) .
$$

Nesta expressão, além destes termos que são explicitamente dependentes da massa, temos ainda $k_{0}=\sqrt{\vec{k}^{2}+m^{2}}$, que nos impede de separar a parte radial da angular na integral dos momentos. Embora essa separação não seja necessária para a finalidade principal do presente estudo, a saber, a comparação entre o limite de altas temperaturas e a equação de transporte de Boltzmann, se quisermos calcular explicitamente as integrais, podemos considerar o limite em que $m \ll T \approx k$, de tal forma que

$$
k=\left(\sqrt{\vec{k}^{2}+m^{2}}, \vec{k}\right)=|\vec{k}|\left(\sqrt{1+\frac{m^{2}}{\vec{k}^{2}}}, \frac{\vec{k}}{|\vec{k}|}\right) \approx|\vec{k}|\left(1, \frac{\vec{k}}{|\vec{k}|}\right) .
$$

Com essa aproximação, toda a dependência na temperatura se reduz ao fator

$$
I^{(n)}(\bar{m})=\int_{0}^{\infty} \frac{d u}{\sqrt{u^{2}+\bar{m}^{2}}} \frac{u^{4-n}}{e^{\sqrt{u^{2}+\bar{m}^{2}}}+1},
$$

em que $\bar{m}=\frac{m}{T}$ (note que o parâmetro $\bar{m}$ pode ser tomado igual a zero para $n=1,2,3$ e serve como um regularizador das integrais com $n \geq 4$; no apêndice $\mathrm{D}$ analisamos o comportamento dessas integrais, usando regularização dimensional).

Assim, podemos escrever (6.14) como

$$
{ }^{ \pm} \Gamma_{\text {transp }}^{(1)}= \pm \frac{e C T^{3} I^{(1)}(\bar{m})}{(2 \pi)^{3}} \int d^{4} x \int d \Omega K_{\mu} A^{\mu}
$$

em que $d \Omega$ representa o elemento de integração nas direções angulares e $I^{(n)}(\bar{m})$ representa a integração sobre $u$. Quando $m \ll T$ podemos usar (6.16), mas em geral a variável $u$ pode estar presente no resultado da integral angular.

Notando que as componentes espaciais da integral acima, $\int d \Omega K_{i}$, são nulas devido à simetria em relação ao intervalo de integração, temos

$$
{ }^{ \pm} \Gamma_{\text {transp }}^{(1)}= \pm \frac{e C T^{3} I^{(1)}(\bar{m})}{2 \pi^{2}} \int d^{4} x A^{0}(x) .
$$


Esse resultado, confirma que para $n=1$, um plasma neutro possuirá corrente e ação efetiva nulas.

Agora, iremos estudar os casos cujas soluções da equação de transporte dependem dos campos externos, ou ao menos, aqueles passíveis de tratamento perturbativo. Para efetuar os cálculos ordem a ordem, um dos métodos mais convenientes envolve a utilização de (6.8), mas ao invés disto, partiremos diretamente para o cálculo da ação efetiva com $n=2$, onde obteremos, através da substituição de (6.3) em (6.4),

$$
{ }^{ \pm} \Gamma_{\text {transp }}^{(n)}=-e^{2} C \int d^{4} x \int d^{4} k A^{\mu} k_{\mu} \frac{1}{k \cdot \partial} k^{\beta} F_{\alpha \beta} \frac{\partial}{\partial k_{\alpha}} f^{(n-2)}(x, \pm k, e) .
$$

O sinal total desta expressão de $\Gamma$, é o mesmo para ambas orientações dos momentos internos, indicando que mesmo no caso de um plasma neutro, se $f^{(n-2)}$ não depender dos sinais dos momentos, a ação efetiva não será nula a priori.

Ainda acerca da expressão (6.19), é possível observar que para $n \geq 2$, haverão interações não triviais entre o plasma e os campos externos. Sendo que os efeitos de todas essas interações, podem ser obtidos através da iteração desta expressão, como será verificado adiante.

Nos próximos passos, efetuaremos algumas manipulações em (6.19), sendo a primeira delas, a integração por partes em relação aos momentos, cujo efeito é eliminar a presença de operadores atuando sobre a função de distribuição.

$$
{ }^{ \pm} \Gamma_{\text {transp }}^{(n)}=e^{2} C \int d^{4} x \int d^{4} k A^{\mu}\left(g^{\alpha}{ }_{\mu} \frac{1}{k . \partial} k^{\beta}-\frac{k_{\mu} k^{\beta} \partial^{\alpha}}{(k . \partial)^{2}}+\frac{k_{\mu} g^{\alpha \beta}}{k . \partial}\right) F_{\alpha \beta} f^{(n-2)}(x, \pm k, e)
$$

Os termos de superfície, provenientes da integração por partes, são nulos devido ao comportamento decrescente da função de Fermi-Dirac para valores tendendo ao infinito. Em (6.20), o termo proporcional à $\delta^{\alpha \beta}$ irá gerar um termo contendo $F_{\alpha \alpha}$, que é nulo devido à antissimetria do tensor eletromagnético.

Em seguida, fazendo uma integração por partes no espaço de configuração, e após algumas redefinições de índices mudos, temos

$$
{ }^{ \pm} \Gamma_{\text {transp }}^{(n)}=e^{2} C \int d^{4} x \int d^{4} k F^{\mu_{2} \nu_{2}} \frac{1}{(k . \partial)^{2}} F^{\mu_{1} \nu_{1}} k_{\mu_{1}} k_{\mu_{2}} g_{\nu_{1} \nu_{2}} f^{(n-2)}(x, \pm k, e),
$$

cujo termo de superfície também é nulo, pelo mesmo motivo já explicitado em (6.20).

A forma da ação efetiva conforme escrita em (6.21), permite uma segunda iteração, e consecutivamente, após outra integração por partes em $k$, culminaremos com uma expressão contendo $f^{(n-3)}$ sem nenhum operador agindo sobre ele. Tal procedimento pode ser aplicado indefinidamente, o que permite calcular a contribuição de qualquer ordem $n$. 
Outra propriedade importante, explicitada em (6.21), é a invariância da ação efetiva por transformações calibre. Para verificar isto, basta notar que $\Gamma$ está escrita em função de tensores eletromagnéticos e de $f^{(n-2)}$, que por sua vez, também depende implicitamente destes tensores, segundo (6.8).

Desse modo, baseando-se nestas duas características da ação efetiva, iremos reescrevê-la no seguinte formato:

${ }^{ \pm} \Gamma_{\text {transp }}^{(n)}=\frac{1}{n !} \int \frac{d^{4} p_{1}}{(2 \pi)^{4}} \ldots \frac{d^{4} p_{n}}{(2 \pi)^{4}} \tilde{A}^{\mu_{1}}\left(p_{1}\right) \ldots \tilde{A}^{\mu_{n}}\left(p_{n}\right){ }^{ \pm} \Pi_{\mu_{1}, \ldots, \mu_{n}}^{\text {transp }}\left(p_{1}, \ldots, p_{n}\right)(2 \pi)^{4} \delta\left(p_{1}+\ldots+p_{n}\right)$,

em que as funções $\tilde{A}^{\mu_{i}}\left(p_{i}\right)$ são as transformadas de Fourier dos campos externos, e ${ }^{ \pm} \Pi$ representa as amplitudes térmicas associadas à equação de transporte de Boltzmann.

A expressão (6.22), além de correlacionar a ação efetiva às amplitudes térmicas de todas as ordens, contém implicitamente as identidades de Ward. Para visualizar isto, basta efetuar uma transformação de calibre através de $\tilde{A}^{\mu_{i}}\left(p_{i}\right) \longrightarrow \tilde{A}^{\mu_{i}}\left(p_{i}\right)+\tilde{\lambda} p_{i}^{\mu_{i}}$, com $\tilde{\lambda}$ constante, e usar a invariância de calibre de (6.22), resultando em

$$
p_{i}^{\mu_{i} \pm} \Pi_{\mu_{1}, \ldots, \mu_{n}}^{\text {transp }}\left(p_{1}, \ldots, p_{n}\right)=0
$$

A forma destas identidades é própria das teorias abelianas, e através delas, deduzimos que cada ordem das amplitudes térmicas é independente das outras ordens e invariante por transformação de calibre individualmente.

Adicionalmente, nota-se através da equação (6.3), que cada iteração diminui em um grau a potência dos momentos internos, e esta mesma potência está diretamente ligada à potência da temperatura. Portanto, para teorias abelianas, cada ordem de aproximação terá uma potência distinta da temperatura.

Dando prosseguimento ao cálculo perturbativo, vamos considerar agora a autoenergia do fóton $(n=2)$, o que nos permitirá verificar os primeiros efeitos das interações com os campos externos.

$$
{ }^{ \pm} \Gamma_{\text {transp }}^{(2)}=e^{2} C \int d^{4} x \int d^{4} k F^{\mu_{2} \nu_{2}} \frac{1}{(k . \partial)^{2}} F^{\mu_{1} \nu_{1}} k_{\mu_{1}} k_{\mu_{2}} g_{\nu_{1} \nu_{2}} f^{(0)}(x, \pm k, e)
$$

A integral em $k_{0}$, e a separação entre as partes radial e angular de $\vec{k}$, podem ser efetuadas de forma análoga à feita para $n=1$.

$$
{ }^{ \pm} \Gamma_{\text {transp }}^{(2)}=e^{2} C \frac{T^{2} I^{(2)}(\bar{m})}{(2 \pi)^{3}} \int d^{4} x \int d \Omega F^{\mu_{2} \nu_{2}} \frac{1}{(k . \partial)^{2}} F^{\mu_{1} \nu_{1}} k_{\mu_{1}} k_{\mu_{2}} g_{\nu_{1} \nu_{2}}
$$

Neste ponto, diferentemente do procedimento assumido no capítulo anterior, prosseguiremos com o tratamento da expressão acima, sendo o próximo passo, substituir os 
campos externos por suas transformadas de Fourier, e consecutivamente, reescreveremos os tensores eletromagnéticos em termos de $\tilde{F}^{\mu_{i} \nu_{i}} \equiv\left(p^{\mu_{i}} \eta^{\nu_{i} \mu}-p^{\nu_{i}} \eta^{\mu_{i} \mu}\right) A_{\mu}$. Além disto, como $p_{1}+p_{2}=0$, fixaremos $p_{1}=p$ e $p_{2}=-p$.

$$
{ }^{ \pm} \Gamma_{\text {transp }}^{(2)}=e^{2} C \frac{T^{2} I^{(2)}(\bar{m})}{(2 \pi)^{3}} \int \frac{d^{4} p}{(2 \pi)^{4}} \int d \Omega \tilde{F}^{\mu_{2} \nu_{2}} \frac{1}{(k \cdot p)^{2}} \tilde{F}^{\mu_{1} \nu_{1}} k_{\mu_{1}} k_{\mu_{2}} g_{\nu_{1} \nu_{2}}
$$

A comparação entre esse resultado para a ação efetiva com a expressão (6.22), permite extrair a amplitude térmica, $\operatorname{com} n=2$, de (6.26).

$$
{ }^{ \pm} \Pi_{\mu \nu}^{\text {transp }}=-e^{2} C \frac{T^{2} I^{(2)}(\bar{m})}{(2 \pi)^{3}} \int d \Omega\left(g_{\mu \nu}-\frac{k_{\mu} p_{\nu}+k_{\nu} p_{\mu}}{k \cdot p}+\frac{p^{2} k_{\mu} k_{\nu}}{(k \cdot p)^{2}}\right)
$$

Após uma análise dimensional desta solução para a auto-energia do fóton, fica claro que temos uma expressão com grau 0 nos momentos externos, fato que se repete para todas as ordens $n$, como é possível verificar em (6.8).

Outra característica presente em (6.27), que é compartilhada por todas as funções de $n$ pontos, é o seu formato, que envolve a presença de uma integral angular, sobre todas as direções de $\widehat{k}=\frac{\vec{k}}{|\vec{k}|}$, e de um tensor produzido pela combinação dos momentos externos com os internos, cuja ordem é $n$. Além disto, a dependência térmica também se repetirá para as diversas ordens de $n$, pois sempre teremos uma potência da temperatura multiplicando a função $I^{(n)}(\bar{m})$.

Essa consistência apontada nesses dois últimos parágrafos, também ocorre para os termos dominantes nos limites de altas temperaturas das teorias não-abelianas, como é observável em (5.30), assim como para teorias de calibre em espaços não comutativos (BRANDT; DAS; FRENKEL, 2002; BRANDT; DAS; FRENKEL, 2003; BRANDT et al., 2002; BRANDT; FRENKEL; MCKEON, 2002).

Por mais uma vez, iremos iteragir a expressão (6.20), assim obtendo ${ }^{ \pm} \Gamma_{\text {transp }}^{(n)}=\mp e^{3} C \int d^{4} x \int d^{4} k F^{\mu_{2} \nu_{2}} \frac{1}{(k . \partial)^{2}} F^{\mu_{1} \nu_{1}} k_{\mu_{1}} k_{\mu_{2}} g_{\nu_{1} \nu_{2}}\left(k_{\beta} \frac{1}{k . \partial} F^{\alpha \beta} \frac{\partial}{\partial k^{\alpha}}\right) f^{(n-3)}(x, \pm k, e)$.

Note, que como $n$ é ímpar, o sinal \pm ressurge multiplicando toda a expressão. Novamente, efetuaremos uma integração por partes no espaço dos momentos, que será seguida de outra integração por partes no espaço de configuração, culminando em

$$
{ }^{ \pm} \Gamma_{\text {transp }}^{(n)}= \pm e^{3} C \int d^{4} x \int d^{4} k F^{\mu_{1} \nu_{1}} \frac{\partial^{\lambda_{1}}}{(k . \partial)^{3}} F^{\mu_{2} \nu_{2}} \frac{\partial^{\lambda_{2}} \partial^{\lambda_{3}}}{(k . \partial)^{3}} F^{\mu_{3} \nu_{3}} T_{\mu_{1} \mu_{2} \mu_{3} \nu_{3} \lambda_{1} \lambda_{2} \lambda_{3}}^{f^{(n-3)}}(x, \pm k, e),
$$

em que os índices $\mu_{3}, \nu_{3}, \lambda_{1}, \lambda_{2}$ e $\lambda_{3}$ são provenientes do rebatismo de alguns índices mudos, e o tensor $T_{\mu_{1} \mu_{2} \mu_{3} \nu_{3} \lambda_{1} \lambda_{2} \lambda_{3}}^{3}$ representa a seguinte combinação dos momentos internos: 
$2 k_{\mu_{1}} k_{\mu_{2}} k_{\mu_{3}} k_{\lambda_{2}} k_{\lambda_{3}} g_{\lambda_{1} \nu_{3}}+k_{\mu_{1}} k_{\mu_{2}} k_{\mu_{3}} k_{\lambda_{1}} k_{\lambda_{2}} g_{\lambda_{3} \nu_{3}}-k_{\mu_{1}} k_{\mu_{3}} k_{\lambda_{1}} k_{\lambda_{2}} k_{\lambda_{3}} g_{\mu_{2} \nu_{3}}-k_{\mu_{2}} k_{\mu_{3}} k_{\lambda_{1}} k_{\lambda_{2}} k_{\lambda_{3}} g_{\mu_{1} \nu_{3}}$.

Agora, com o auxílio de (6.29) e (6.30), será possível calcular a amplitude térmica para $n=3$, bastando substituir os campos externos por suas transformadas de Fourier, e utilizar (6.22) para identificar a amplitude térmica na ação efetiva.

$$
{ }^{ \pm} \Pi_{\mu_{1} \mu_{2} \mu_{3}}^{\text {transp }}= \pm 6 e^{3} C \frac{T I^{(3)}(\bar{m})}{(2 \pi)^{3}} \int d \Omega \mathcal{A}_{\mu_{1} \mu_{2} \mu_{3}}
$$

A expressão para $\mathcal{A}_{\mu_{1} \mu_{2} \mu_{3}}$ por ser muito longa, encontra-se escrita no apêndice E, onde também constam os resultados para $n=4$ e uma descrição do algoritmo utilizado em todos estes cálculos, já que a quantidade de termos, que ocorrem nos procedimentos desenvolvidos nesta seção, aumenta expressivamente conforme prosseguimos com a ordem de aproximação.

De forma geral, podemos concluir que a utilização da equação de transporte de Boltzmann permite encontrar expressões tanto para a ação efetiva, quanto para as amplitudes térmicas, isto para qualquer que seja $n$. E ainda, que essas duas quantidades compartilham termos do seguinte tipo:

$$
\frac{( \pm e)^{n}}{(2 \pi)^{3}} T^{4-n} I^{(n)}(\bar{m}) C \int d \Omega \mathcal{A}_{\mu_{1}, \ldots, \mu_{3}}
$$

\subsection{Limite de altas temperaturas da QED}

Em contrapartida à seção anterior, nesta iremos utilizar uma teoria quântica nos cálculos da ação efetiva e das amplitudes térmicas para um plasma de férmions. Sendo assim, dentre as opções possíveis para incluir os efeitos térmicos na QED, utilizaremos o já apresentado formalismo do tempo imaginário, pois este permite que se obtenha as amplitudes térmicas de forma direta, como foi visto no capítulo 2.

Porém, antes de partimos diretamente para o cálculo das funções de n pontos, precisamos definir as regras de Feynman para a QED. Todavia, elas podem ser obtidas facilmente ao particularizarmos as relações (3.45a), (3.45b) e (3.46) para o caso abeliano, 
resultando em

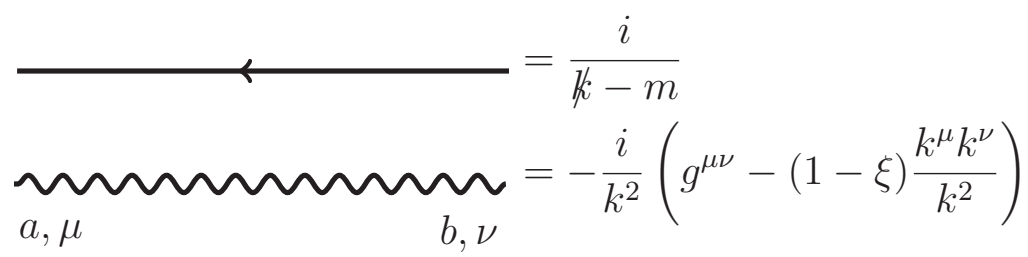

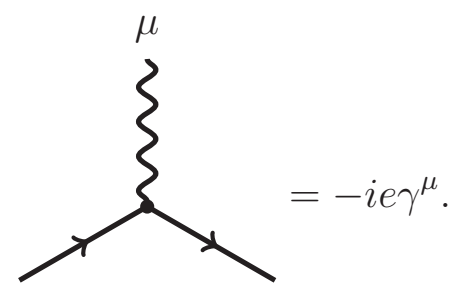

Nessas regras acima, as linhas contínuas representam férmions com frequência de Matsubara $w_{n}=(2 n+1) \pi T$, e as linhas onduladas são fótons com frequência $w_{n^{\prime}}=2 n^{\prime} \pi T$.

Adicionalmente, para manter uma equivalência com a seção anterior, na qual consideramos férmions térmicos interagindo com fótons externos, nesta iremos utilizar um plasma, cujas interações são representadas por diagramas de Feynman compostos por um loop fermiônico e n pernas externas bosônicas.

Esses diagramas de $\mathrm{n}$ fótons, figura 15 , representam apenas uma das $(n-1)$ ! possibilidades de organizarmos os fótons, pernas externas, ao redor do loop fermiônico. No entanto, não será necessário efetuar os cálculos para cada um desses diagramas individualmente, pois basta obter a expressão para um único caso, e então aplicar as permutações dos fótons. Ademais, este procedimento irá garantir uma simetria bosônica ao resultado, esta que já era esperada, pois esses diagramas referem-se às amplitudes térmicas dos fótons.

Assim, aplicando as regras de Feynman ao diagrama da figura 15, temos

$$
G_{\mu_{1}, \ldots, \mu_{n}}=-T \sum_{k_{0}=i \omega_{n}} \int \frac{d^{3} k}{(2 \pi)^{3}} f_{\mu_{1}, \ldots, \mu_{n}}\left(k_{0}, \vec{k}\right),
$$

em que o sinal negativo é devido ao loop fermiônico, e

$$
f_{\mu_{1}, \ldots, \mu_{n}}\left(k_{0}, \vec{k}\right)=\operatorname{Tr}\left[\frac{1}{\not k-m} \gamma_{\mu_{1}} \frac{1}{\not k+\not p_{1}-m} \cdots \gamma_{\mu_{n}} \frac{1}{\not k-\not p_{n}-m}\right] \text {. }
$$

Nesta expressão, assim como na figura 15, o sinal de $p_{n}$, que é contrário aos outros momentos externos, visa garantir a conservação dos momentos.

Além disso, para esses fatores contidos em (6.35), é possível reescrever os propagadores fermiônicos de forma a manter as matrizes gama no numerador ${ }^{1}$, e consequentemente,

$$
\frac{1}{\not k+\not p_{i}-m}=\frac{1}{\not k+\not p_{i}-m} \frac{\not k+\not p_{i}+m}{\not k+\not p_{i}+m}=\frac{\not k+\not p_{i}+m}{\left(k+p_{i}\right)^{2}-m^{2}}
$$


obteremos denominadores com polos em $\pm \sqrt{\vec{k}^{2}+m^{2}}, \pm \sqrt{\left(\vec{k}+p_{1}\right)^{2}+m^{2}}$, e assim por diante. Estes polos, como vimos na seção 2.3, irão garantir, que as partículas pertencentes ao loop estarão na camada de massa.

Novamente, utilizaremos (2.57) para transformar a soma nas frequências de Matsubara em uma integral no plano complexo,

$$
G_{\mu_{1}, \ldots, \mu_{n}}^{\text {therm }}=\int \frac{d^{3} k}{(2 \pi)^{3}} \int_{-i \infty+\delta}^{i \infty+\delta} \frac{d k_{0}}{2 \pi i} N_{F}\left(k_{0}\right)\left[f_{\mu_{1}, \ldots, \mu_{n}}\left(k_{0}, \vec{k}\right)+f_{\mu_{1}, \ldots, \mu_{n}}\left(-k_{0}, \vec{k}\right)\right] .
$$

Nesta última passagem, omitimos o termo contendo os efeitos respectivos ao vácuo, pois além de não depender da temperatura, ele também é puramente quântico, e por isto esperamos que ele não contribua, quando efetuado o limite para um sistema clássico, sendo assim, daqui por diante ignoraremos os termos com temperatura nula.

De forma análoga à separação feita em (6.9), entre as contribuições dos elétrons e dos pósitrons, aplicaremos uma mudança de variáveis $\vec{k} \rightarrow-\vec{k}$ no segundo termo de (6.36), e definiremos

$$
{ }^{ \pm} G_{\mu_{1}, \ldots, \mu_{n}}^{\text {therm }}=\int \frac{d^{3} k}{(2 \pi)^{3}} \int_{-i \infty+\delta}^{i \infty+\delta} \frac{d k_{0}}{2 \pi i} N_{F}\left(k_{0}\right) f_{\mu_{1}, \ldots, \mu_{n}}( \pm k)
$$

sendo que $G={ }^{+} G+{ }^{-} G$.

Após efetuada a integração em $k_{0}$ na expressão acima, aplicaremos uma continuação analítica para o espaço de Minkowski, o que nos levará às regras de Feynman da QED com temperatura nula. Desta forma, como as energias dos fótons externos assumirão valores contínuos, poderemos tratar o plasma em um regime fora da zona de equilíbrio.

$$
{ }^{ \pm} G_{\mu_{1}, \ldots, \mu_{n}}^{\text {therm }}=-\int \frac{d^{3} k}{(2 \pi)^{3}} \frac{N_{F}\left(\sqrt{\vec{k}^{2}+m^{2}}\right)}{2 \sqrt{\vec{k}^{2}+m^{2}}} \sum_{\text {cíclica }} \mathcal{A}_{\mu_{1}, \ldots, \mu_{n}}^{n}\left( \pm k, p_{1}, \ldots, p_{n}\right),
$$

em que os fatores $\mathcal{A}_{\mu_{1} \cdots \mu_{n}}^{n}$ contêm as expressões das amplitudes frontais, que podem ser representadas diagramaticamente como exposto na figura 16.

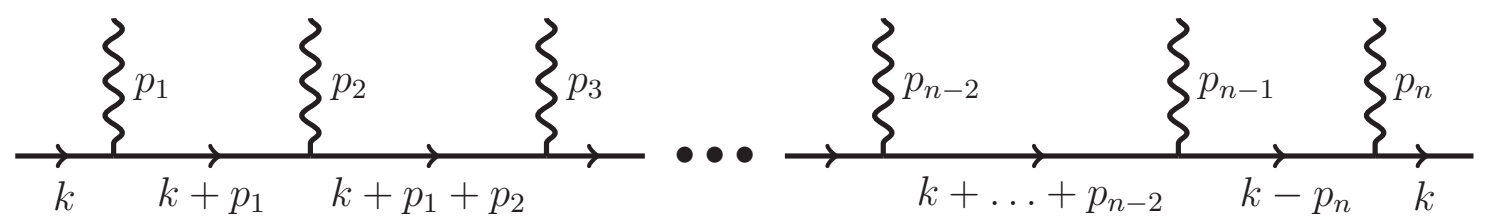

Figura 16 - Amplitude frontal de um férmion térmico interagindo com $n$ fótons externos.

Ademais, podemos interpretar, que esses diagramas se referem a um espalhamento de férmions térmicos, na camada de massa, por fótons pertencentes ao plasma. Tal interpretação foi previamente discutida por Barton(BARTON, 1990), e equivale à discussão efetuada na seção 2.3. 
Retornando às amplitudes frontais contidas em (6.38), e aproveitando os diagramas da figura 16, podemos escrevê-las mais especificamente como

$$
\mathcal{A}_{\mu_{1}, \ldots, \mu_{n}}^{n}\left(k, p_{1}, \ldots, p_{n}\right)=-e^{n} \operatorname{Tr}\left[\gamma_{\alpha_{1}} \gamma_{\mu_{1}} \ldots \gamma_{\alpha_{n}} \gamma_{\mu_{n}}\right] \frac{k^{\alpha_{1}} \ldots\left(k+S_{n-1}\right)^{\alpha_{n}}}{\left(p_{1}^{2}+2 k \cdot p_{1}\right) \ldots\left(S_{n-1}^{2}+2 k . S_{n-1}\right)},
$$

em que $S_{n-1}=p_{1}+p_{2}+\ldots+p_{n-1}$, para $n \geq 2$.

O sinal negativo, que ocorre em (6.39) é devido à continuação analítica. Além disso, após calculados os traços contidos nos fatores $\mathcal{A}_{\mu_{1} \cdots \mu_{n}}^{n}$, e utilizando o fato de que os férmions encontram-se na camada de massa, observaremos um anulamento dos termos proporcionais às potências pares da massa.

Adicionalmente, os termos proporcionais às potências ímpares da massa, serão nulos para dimensões pares do espaço-tempo, pois nesta condição, teremos traços de um número ímpar de matrizes gama. No entanto, se o espaço-tempo possuir dimensão ímpar, ocorrerão termos de Chern-Simons induzidos(CHERN; SIMONS, 1974; DESER; JACKIW; TEMPLETON, 1982). Portanto, para a QED no espaço-tempo com $3+1$ dimensões, não existirá no numerador, nenhum termo proporcional à massa.

Posto isto, devemos notar que a expressão (6.38), juntamente com (6.39), define o resultado para apenas um dos $(n-1)$ ! diagramas que podem ser derivados da figura 16 . Portanto, a fim de obter o resultado completo para as amplitudes térmicas, deveremos somar todas as $(n-1)$ ! permutações das pernas externas.

$$
{ }^{ \pm} \Pi_{\mu_{1}, \ldots, \mu_{n}}^{\text {therm }}=-\int \frac{d^{3} k}{(2 \pi)^{3}} \frac{N_{F}\left(\sqrt{\vec{k}^{2}+m^{2}}\right)}{2 \sqrt{\vec{k}^{2}+m^{2}}} \sum_{\text {cíclica }} \sum_{\substack{(n-1) ! \\ \text { pernas }}} \mathcal{A}_{\mu_{1}, \ldots, \mu_{n}}^{n}\left( \pm k, p_{1}, \ldots, p_{n}\right)
$$

Ao compararmos este resultado com o obtido na seção anterior em (6.32), nota-se que ambas expressões possuem em comum a função $\frac{N_{F}\left(\sqrt{\vec{k}^{2}+m^{2}}\right)}{2 \sqrt{\vec{k}^{2}+m^{2}}}$, fator que domina na região ultravioleta o comportamento da integral nos momentos internos.

Porém, diferentemente do que ocorreu quando utilizamos a equação de transporte, não será possível reescrever os termos $\mathcal{A}_{\mu_{1} \cdots \mu_{n}}^{n}$ como uma função do tipo $|\vec{k}|^{\alpha} \bar{A}_{\mu_{1} \cdots \mu_{n}}^{n}$, onde $\alpha$ é uma potência inteira, e $\bar{A}_{\mu_{1} \cdots \mu_{n}}^{n}$ é uma função que depende apenas da parte angular do quadrivetor $k$. Então, (6.40) não possuirá uma dependência térmica simples do tipo $I^{(n)}(\bar{m})$, como ocorreu para $\pm \Pi_{\mu_{1} \cdots \mu_{n}}^{\text {transp }}$.

A diferença apontada no parágrafo anterior, ocorre devido a efeitos quânticos, que estão presentes apenas em $\Pi_{\mu_{1} \cdots \mu_{n}}^{\text {therm }}$. Desta forma, para haver uma equivalência entre os dois formalismos, será necessário aplicar o limite de altas temperaturas, pois este conduzirá a amplitude $\Pi_{\mu_{1} \cdots \mu_{n}}^{\text {therm }}$ para uma região clássica, conforme argumentado no Capítulo 4, e assim 
obteremos uma amplitude $\prod_{\mu_{1} \cdots \mu_{n}}^{h t l}$ para altas temperaturas, que irá gerar a seguinte ação efetiva:

${ }^{ \pm} \Gamma_{h t l}^{(n)}=\frac{1}{(n-1) !} \int \frac{d^{4} p_{1}}{(2 \pi)^{4}} \ldots \frac{d^{4} p_{n}}{(2 \pi)^{4}} \tilde{A}^{\mu_{1}}\left(p_{1}\right) \ldots \tilde{A}^{\mu_{n}}\left(p_{n}\right){ }^{ \pm} \Pi_{\mu_{1}, \ldots, \mu_{n}}^{h t l}\left(p_{1}, \ldots, p_{n}\right)(2 \pi)^{4} \delta\left(p_{1}+\ldots+p_{n}\right)$.

Agora, cumprindo com o intuito deste capítulo, que é investigar se esta ação efetiva acima equivale à presente em (6.22), para todas as ordens de $n$, e para um plasma com distribuição de cargas genérica, iremos comparar (6.41) com (6.22).

$$
{ }^{ \pm} \prod_{\mu_{1}, \ldots, \mu_{n}}^{h t l}\left(p_{1}, \ldots, p_{n}\right)=\frac{1}{n}{ }^{ \pm} \Pi_{\mu_{1}, \ldots, \mu_{n}}^{\text {transp }}\left(p_{1}, \ldots, p_{n}\right)
$$

Todavia, antes de prosseguir com a comparação entre os termos explícitos destas amplitudes térmicas, ainda é necessário efetuar o limite de altas temperaturas em (6.40). Para isto, consideraremos novamente, o limite em que $k_{\mu} \gg p_{\mu}^{\prime}$, sendo $p_{\mu}^{\prime}$ qualquer um dos momentos externos, e expandiremos os denominadores das amplitudes frontais como

$$
\frac{1}{p_{n}^{2} \pm 2 k \cdot p_{n}}=\frac{1}{2 k \cdot p_{n}\left(1 \pm \frac{p_{n}^{2}}{2 k \cdot p_{n}}\right)} \approx \frac{1}{2 k \cdot p_{n}} \mp \frac{p_{n}^{2}}{\left(2 k \cdot p_{n}\right)^{2}}+\ldots
$$

Reescrevendo novamente $k_{\mu}=|\vec{k}| K_{\mu}$, com $K=\left(1, \frac{\vec{k}}{|\vec{k}|}\right)$, conforme (6.15), e combinando a expansão acima com os numeradores de (6.39), temos

$\mathcal{A}_{\mu_{1}, \ldots, \mu_{n}}^{n}\left( \pm|\vec{k}| K, p_{1}, \ldots, p_{n}\right)= \pm|\vec{k}| \mathcal{A}_{1_{\mu_{1}, \ldots, \mu_{n}}}^{n}+\mathcal{A}_{2 \mu_{1}, \ldots, \mu_{n}}^{n} \pm \frac{1}{|\vec{k}|} \mathcal{A}_{3 \mu_{1}, \ldots, \mu_{n}}^{n}+\frac{1}{|\vec{k}|^{2}} \mathcal{A}_{4_{\mu_{1}, \ldots, \mu_{n}}}^{n} \ldots$

Estas funções $\mathcal{A}_{1 \mu_{1} \cdots \mu_{n}}^{n}, \mathcal{A}_{2 \mu_{1} \cdots \mu_{n}}^{n}$, etc, dependerão apenas das direções dos momentos internos e externos, pois a expansão feita no limite HTL, permitiu fatorar a parte radial de $k$. Além disto, a expansão apresentada em (6.44) é finita e limitada até os termos do tipo $\mathcal{A}_{n}^{n}$.

A princípio, a expressão (6.44) irá produzir, para um determinado $n$, termos com diferentes potências da temperatura, o que contraria os resultados da seção anterior. No entanto, como veremos, ocorrerão alguns anulamentos, de forma a restar apenas uma potência da temperatura para cada uma das amplitudes.

O caso mais simples, $n=1$, não evidencia o uso do limite HTL, pois $\mathcal{A}_{\mu}^{1}$ não possui denominador, e consecutivamente, nenhuma singularidade.

$$
\mathcal{A}_{\mu}^{1}( \pm|\vec{k}| K)=\mp e|\vec{k}| \operatorname{Tr}\left[\not K \gamma_{\mu}\right]=\mp 4 e|\vec{k}| K_{\mu}
$$


A substituição de (6.45) em (6.40), com ou sem o limite HTL, irá gerar o mesmo resultado,

$$
\begin{aligned}
{ }^{ \pm} \Pi_{\mu}^{t h e r m}={ }^{ \pm} \Pi_{\mu}^{h t l} & = \pm \frac{2 e}{(2 \pi)^{3}} \int_{0}^{\infty} d|\vec{k}||\vec{k}|^{3} \frac{N_{F}\left(\sqrt{\vec{k}^{2}+m^{2}}\right)}{2 \sqrt{\vec{k}^{2}+m^{2}}} \int d \Omega K_{\mu} \\
& = \pm \frac{2 e}{(2 \pi)^{3}} T^{3} I^{(1)}(\bar{m}) \int d \Omega K_{\mu} .
\end{aligned}
$$

Na última passagem acima, utilizamos $u T=|\vec{k}|, \bar{m}=\frac{m}{T}$ e $I^{(n)}(\bar{m})$, conforme definido na seção anterior. Este resultado (6.46), irá reproduzir a ação efetiva (6.18) se feita a identificação $C=2$, que já era esperada para férmions em $3+1$ dimensões, pois este valor equivale aos dois graus de liberdade do spin eletrônico.

Dando continuidade à comparação entre os dois formalismos, prosseguiremos para uma ordem de aproximação superior, $n=2$, na qual devemos calcular $\mathcal{A}_{1}^{2}$ e $\mathcal{A}_{2}^{2}$. O primeiro destes fatores é proporcional à $T^{3} I^{(1)}(\bar{m})$, todavia, esperamos que a função de dois pontos seja proporcional à $T^{2} I^{(2)}(\bar{m})$.

Portanto, $\mathcal{A}_{1}^{2}$ não deverá contribuir com a amplitude $\Pi_{\mu \nu}^{h t l}$. Aliás, de forma geral, todos termos do tipo $\mathcal{A}_{1}^{n}$, com $n>1$, devem ser nulos por este mesmo motivo. Para demonstrar este fato, devemos primeiramente, observar que estes termos sempre contêm as maiores potências de $k$ no numerador, isto é, eles são sempre proporcionais ao seguinte tipo de fatores

$$
\frac{K_{\alpha_{1}} K_{\alpha_{2}} \ldots K_{\alpha_{n}}}{\left(K . p_{1}\right)\left[K .\left(p_{1}+p_{2}\right)\right] \ldots\left[K .\left(p_{1}+p_{2}+\ldots+p_{n-1}\right)\right]}
$$

Posto isto, ao somarmos todas as permutações cíclicas destes fatores, e utilizando a conservação de momento, concluiremos que os termos $\mathcal{A}_{1}^{n}$ serão nulos, a menos do caso $n=1$.

$$
\sum_{\text {cíclica }} \mathcal{A}_{1}^{n}{ }_{\mu_{1}, \ldots, \mu_{n}}\left(K, p_{1}, \ldots, p_{n}\right)=0 ; n \geq 2
$$

Como bastou somar apenas as permutações cíclicas, não sendo necessária a soma das permutações das pernas externas, e muito menos impor que o plasma fosse neutro, esta propriedade é geral, e ocorre individualmente para cada um dos diagramas representados na figura 15.

Resta agora, o cálculo do termo proporcional à $T^{2}$, que representa a autoenergia do fóton. Após poucos cálculos e algumas simplificações, obtemos

$$
\mathcal{A}_{2 \mu \nu}^{2}=2 e^{2}\left(g_{\mu \nu}-\frac{K_{\mu} p_{\nu}+K_{\nu} p_{\mu}}{K \cdot p}+\frac{p^{2} K_{\mu} K_{\nu}}{(K \cdot p)^{2}}\right) .
$$


A inserção desta equação em (6.40) tem como resultado:

$$
{ }^{ \pm} \Pi_{\mu \nu}^{h t l}=-\frac{2 e^{2}}{(2 \pi)^{3}} T^{2} I^{(2)}(\bar{m}) \int d \Omega\left(g_{\mu \nu}-\frac{K_{\mu} p_{\nu}+K_{\nu} p_{\mu}}{K \cdot p}+\frac{p^{2} K_{\mu} K_{\nu}}{(K \cdot p)^{2}}\right) .
$$

Novamente, concluímos que $C$ deve ser igual a 2 ao compararmos (6.50) com (6.27). Já para as ordens superiores, $n \geq 3$, assim como na seção anterior, utilizaremos o pacote de cálculos algébricos FeynCalc.

Na ordem $n=3$, além do termo proporcional à $T^{3}$, que é nulo conforme (6.48), existem outros dois, um deles proporcional à $T^{2}$ e outro à $T$. Para o termo quadrático na temperatura, temos a expressão abaixo, que foi obtida via rotina apresentada no apêndice E. É notável, que (6.51) é antissimétrico na permutação das pernas externas, e assim ao somarmos todas as suas permutações cíclicas, ocorrerão alguns cancelamentos triviais culminando em um resultado nulo para $\mathcal{A}_{2}^{3}$.

$$
\mathcal{A}_{2 \mu_{1} \mu_{2} \mu_{3}}^{3}=\frac{2 e^{3} K_{\mu_{3}}}{K \cdot p_{3}}\left(g_{\mu_{1} \mu_{2}}-\frac{K_{\mu_{1}} p_{\mu_{\mu_{2}}}+K_{\mu_{2}} p_{1_{\mu_{1}}}}{K \cdot p_{1}}+\frac{p_{1}^{2} K_{\mu_{1}} K_{\mu_{2}}}{\left(K \cdot p_{1}\right)^{2}}\right)-\left(\mu_{1}, p_{1}\right) \leftrightarrow\left(\mu_{3}, p_{3}\right)
$$

Por fim, devemos calcular a contribuição dos fatores $\mathcal{A}_{3 \mu_{1} \cdots \mu_{n}}^{3}$ para $\prod_{\mu_{1} \mu_{2} \mu_{3}}^{h t l}$. O resultado e a comparação, deste termo dominante, com o obtido em (6.31), estão apresentados no apêndice $\mathrm{E}$, onde novamente encontramos uma equivalência entre os dois formalismos, se $C=2$.

Também no apêndice E, vemos que assim como ocorreu para todas as ordens anteriores, quando consideramos $n=4$, encontramos uma equivalência entre o formalismo da equação de transporte de Boltzmann e o limite HTL da QED com temperatura finita, considerando $C=2$. Para chegar a tal conclusão, observamos que existe um cancelamento das contribuições provenientes dos fatores $\mathcal{A}_{2 \mu_{1} \cdots \mu_{n}}^{4}$ e $\mathcal{A}_{3 \mu_{1} \cdots \mu_{n}}^{4}$, após efetuada a somatória de todas as permutações. Além destes dois termos, existe o proporcional à $T^{3}$, que mostramos ser nulo através da equação (6.48).

Neste capítulo, demonstramos através de cálculos explícitos, que até a ordem $n=4$, existe uma equivalência entre o limite de altas temperaturas da QED e a equação de transporte de Boltzmann. Tal equivalência, é evidenciada através da comparação entre as amplitudes térmicas resultantes de cada formalismo.

Adicionalmente, mostramos que a dependência térmica destas amplitudes, não é representada apenas por uma potência da temperatura, mas de uma função cujo parâmetro contém a relação direta entre massa e temperatura, $I^{(n)}(\bar{m})$. 



\section{Conclusão}

No presente trabalho investigamos a conexão entre o formalismo de equação de transporte de Boltzmann, e o limite de altas temperaturas da TQFT. Para tal, assumimos que ambas as teorias geram uma mesma ação efetiva, que por sua vez pode ser expandida em termos de funções de $n$ pontos. Desta forma, para verificar a equivalência entre os dois formalismos, comparamos as amplitudes térmicas obtidas por cada um deles.

Além disso, vimos que não é possível obter uma expressão explícita e geral para essas amplitudes quando obtidas via TQFT, o que nos impede de efetuar essa comparação para uma ordem de aproximação arbitrária. Sendo assim, foi necessário especificar as ordens de aproximação para as quais efetuamos nossos cálculos, que foram iniciados pelo caso de uma teoria de calibre não-abeliana com dimensão espaço-temporal e grupo de simetria genéricos. Assim sendo, após cálculos explícitos até a segunda ordem de aproximação, foi possível estabelecer e concluir que ambos os formalismos concordam no limite de altas temperaturas.

Todavia, com o intuito de demonstrar uma conexão ainda mais profunda, escolhemos uma teoria de calibre abeliana, mais especificamente a QED, que propiciou manter a rastreabilidade da dependência térmica de cada ordem de aproximação isoladamente. Adicionalmente, consideramos também uma distribuição de cargas genérica, e assim pudemos analisar as amplitudes com um número ímpar de pernas externas, pois se tivéssemos utilizado um plasma neutro, tais contribuições seriam nulas. Posto isto, foram efetuados cálculos explícitos até a quarta ordem de aproximação, que devido à grande quantidade de termos, exigiu a utilização de uma rotina computacional. Concluímos então que os formalismos, clássico e quântico, concordam no limite de altas temperaturas.

Adicionalmente, no decorrer do estudo, vimos que o formalismo da equação de Boltzmann, permite que identifiquemos o comportamento térmico dos termos dominantes, para qualquer que seja a ordem de iteração, o que não ocorre para o limite de altas temperaturas da TQFT. Somado à este fato, ainda tivemos que demonstrar a nulidade de vários termos obtidos via HTL, sendo que estes aumentam em quantidade, em ordens superiores de aproximação. Portanto, o formalismo clássico permite obter de forma mais direta as amplitudes térmicas com um número arbitrário de fótons externos.

Em contrapartida, se considerarmos um certo modelo físico descrito pela TQFT, desde que as condições para impor o HTL sejam cumpridas, sempre poderemos obter o limite de altas temperaturas deste sistema físico, assim culminando nas amplitudes térmicas que seriam obtidas via um sistema clássico, sendo este equivalente ao limite de altas temperaturas do nosso modelo quântico inicial. Mesmo que esse procedimento não 
permita obter de forma direta as equações de movimento deste sistema clássico, ao menos saberíamos o resultado das interações de seus termos, e assim, após uma certa engenharia reversa, talvez fosse possível recuperar essas equações que regem o comportamento do referido sistema. No entanto, um procedimento contrário a este não é possível, pois a teoria quântica é muito mais rica em detalhes e interações, cuja fineza de seus efeitos é perdida ao impormos o limite de altas temperaturas.

Por fim, toda essa conexão demonstrada entre a equação de transporte e o limite de altas temperaturas da TQFT, se deu para um plasma em que desconsideramos os efeitos das colisões. Como resultado prático, foi necessário somente considerar os diagramas com apenas um loop, que resultam em amplitudes frontais representadas por um férmion térmico interagindo com $n$ fótons ou n bósons de calibre pertencentes ao plasma. Diante disso, é natural imaginar que, talvez, as próximas correções quânticas possam descrever os termos de colisão, e portanto, necessitaríamos considerar também os diagramas de dois ou mais loops. Esse problema está em aberto, e configura uma desafiadora oportunidade de pesquisa. 


\section{APÊNDICE A - Ação efetiva}

Como alternativa ao formalismo desenvolvido na seção 2.2, onde expomos como extrair os diagramas de Feynman da função de partição, demonstraremos um método baseado em funcionais geradores. Para entendermos o papel desses funcionais, vamos inicialmente estudar uma generalização da função de partição,

$$
Z[J]=\int[d \phi] e^{i \int d^{4} x[\mathcal{L}+J(x) \phi(x)]} .
$$

Esse funcional, definido para campos escalares, que aqui usamos por simplicidade, contém um termo a mais na ação, que representa uma fonte externa, e por isto é denominado termo fonte. Ademais, a finalidade prática deste novo termo, pode ser explicitada no seguinte cálculo:

$$
\frac{\delta}{\delta J(x)} Z[J]=\int[d \phi] \frac{\delta}{\delta J(x)} e^{i \int d^{4} x^{\prime}\left[\mathcal{L}+J\left(x^{\prime}\right) \phi\left(x^{\prime}\right)\right]}=\int[d \phi] i \phi(x) e^{i \int d^{4} x^{\prime}\left[\mathcal{L}+J\left(x^{\prime}\right) \phi\left(x^{\prime}\right)\right]} .
$$

Na expressão acima vemos que a aplicação do operador $\frac{\delta}{\delta J}^{1}$ no funcional gerador $Z$, irá agir na exponencial do termo fonte, resultando na extração de uma potência do campo escalar.

Ao combinarmos as expressões (A.1) e (A.2), é possível criar um procedimento que obtém a média de qualquer potência dos campos a partir da função de partição,

$$
\begin{array}{r}
\left.\frac{1}{Z[0]}\left(-i \frac{\delta}{\delta J\left(x_{1}\right)}\right)\left(-i \frac{\delta}{\delta J\left(x_{2}\right)}\right) \ldots\left(-i \frac{\delta}{\delta J\left(x_{n}\right)}\right) Z[J]\right|_{J=0}= \\
\frac{1}{Z[0]} \int[d \phi] \phi x_{1} \phi\left(x_{2}\right) \ldots \phi\left(x_{n}\right) e^{i \int d^{4} x^{\prime}[\mathcal{L}]}=\left\langle T_{\tau} \phi\left(x_{1}\right) \phi\left(x_{2}\right) \ldots \phi\left(x_{n}\right)\right\rangle,
\end{array}
$$

em que $T_{\tau}$ é o operador de ordenação temporal.

Além disto, vemos através de (A.5), que o funcional (A.1) é capaz de gerar todas as funções de correlação para uma determinada teoria, que por sua vez estão relacionadas a todos os tipos de diagramas, desde os desconexos até os 1PI.

1 Podemos definir a derivação funcional, através da seguinte equação:

$$
\frac{\delta}{\delta J(x)} J(y)=\delta^{(n)}(x-y)
$$

em que $n$ é a dimensão do espaço-tempo. Com o auxílio desta relação e da regra para derivação de funções compostas, podemos efetuar a derivada de funcionais mais complexos, por exemplo,

$$
\frac{\delta}{\delta J(x)} e^{i \alpha \int d^{n} y J(y) f(y)}=i \alpha f(x) e^{i \alpha \int d^{n} y J(y) f(y)} .
$$


Inspirados por (B.22), que associa o logaritmo da função de partição com os diagramas próprios, vamos definir um funcional $W[J]$ que obedece a seguinte relação:

$$
Z[J]=e^{i W[J]} .
$$

Agora, com o intuito de analisar a ação do operador $\frac{\delta}{\delta J}$ em $W[J]$, iremos aplicá-lo em ambos os lados da equação acima, e em seguida, cessaremos a fonte externa,

$$
\begin{aligned}
\left.\frac{\delta}{\delta J\left(x_{1}\right)} Z[J]\right|_{J=0}=\frac{\delta}{\delta J\left(x_{1}\right)} e^{i W[J]} & =\left.i\left[\frac{\delta}{\delta J\left(x_{1}\right)} W[J]\right] e^{i W[J]}\right|_{J=0}=\left.i Z[0]\left[\frac{\delta}{\delta J\left(x_{1}\right)} W[J]\right]\right|_{J=0} \\
\left.\frac{\delta}{\delta J\left(x_{1}\right)} W[J]\right|_{J=0} & =-\left.i \frac{1}{Z[0]} \frac{\delta}{\delta J\left(x_{1}\right)} Z[J]\right|_{J=0}=\left\langle\phi\left(x_{1}\right)\right\rangle .
\end{aligned}
$$

Embora essa média $\left\langle\phi\left(x_{1}\right)\right\rangle$ seja nula, aqui fica claro que a derivação funcional de $W[J]$ gera diretamente a média de uma potência dos campos. Para compreender melhor essa relação, agora iremos aplicar o operador $\frac{\delta}{\delta J}$ duas vezes em (A.6), e novamente, extinguir os efeitos da fonte externa,

$$
\begin{aligned}
& \left.\frac{\delta}{\delta J\left(x_{1}\right)} \frac{\delta}{\delta J\left(x_{2}\right)} Z[J]\right|_{J=0}=\left.\frac{\delta^{2}}{\delta J\left(x_{1}\right) \delta J\left(x_{2}\right)}\left[e^{i W[J]}\right]\right|_{J=0}=\left.\frac{\delta}{\delta J\left(x_{1}\right)}\left[i \frac{\delta}{\delta J\left(x_{2}\right)} W[J]\right] e^{i W[J]}\right|_{J=0}= \\
& =\left.Z[0]\left[i \frac{\delta^{2}}{\delta J\left(x_{1}\right) \delta J\left(x_{2}\right)} W[J]\right]\right|_{J=0}+\left.Z[0]\left[i \frac{\delta}{\delta J\left(x_{2}\right)} W[J] i \frac{\delta}{\delta J\left(x_{1}\right)} W[J]\right]\right|_{J=0} .
\end{aligned}
$$

Ao isolarmos a derivação funcional dupla de $W[J]$ na expressão acima, juntamente com o resultado (A.7), obtemos

$$
\begin{aligned}
\left.\frac{\delta^{2}}{\delta J\left(x_{1}\right) \delta J\left(x_{2}\right)} W[J]\right|_{J=0} & =-\left.i \frac{1}{Z[0]} \frac{\delta}{\delta J\left(x_{1}\right)} \frac{\delta}{\delta J\left(x_{2}\right)} Z[J]\right|_{J=0}-i\left\langle\phi\left(x_{2}\right)\right\rangle\left\langle\phi\left(x_{1}\right)\right\rangle \\
& =i\left(\left\langle\phi\left(x_{2}\right) \phi\left(x_{1}\right)\right\rangle-\left\langle\phi\left(x_{2}\right)\right\rangle\left\langle\phi\left(x_{1}\right)\right\rangle\right) .
\end{aligned}
$$

Desconsiderando por um momento que $\langle\phi(x)\rangle$ é nulo, vemos que o último termo a direita desta expressão, desconta de $\left\langle\phi\left(x_{2}\right) \phi\left(x_{1}\right)\right\rangle$ seus diagramas desconexos. Aliás, o mesmo ocorre para a próxima ordem, onde temos

$$
\begin{gathered}
\left.\frac{\delta^{3}}{\delta J\left(x_{1}\right) \delta J\left(x_{2}\right) \delta J\left(x_{3}\right)} W[J]\right|_{J=0}=i^{2}\left(\left\langle\phi\left(x_{1}\right) \phi\left(x_{2}\right) \phi\left(x_{3}\right)\right\rangle-\left\langle\phi\left(x_{1}\right) \phi\left(x_{2}\right)\right\rangle\left\langle\phi\left(x_{3}\right)\right\rangle+\right. \\
\left.-\left\langle\phi\left(x_{1}\right) \phi\left(x_{3}\right)\right\rangle\left\langle\phi\left(x_{2}\right)\right\rangle-\left\langle\phi\left(x_{2}\right) \phi\left(x_{3}\right)\right\rangle\left\langle\phi\left(x_{1}\right)\right\rangle+2\left\langle\phi\left(x_{1}\right)\right\rangle\left\langle\phi\left(x_{2}\right)\right\rangle\left\langle\phi\left(x_{3}\right)\right\rangle\right),
\end{gathered}
$$

e assim, sucessivamente, para todas as ordens superiores. 
Portanto, $W[J]$ é o funcional gerador das funções de correlação conexas, fato já demonstrado na seção B, onde foram utilizadas diretamente as expressões explícitas de $\ln (Z)$. Adicionalmente, podemos generalizar (A.10) como

$$
\frac{\delta^{n} W[J]}{\delta J\left(x_{1}\right) \ldots \delta J\left(x_{n}\right)}=i^{n-1}\left\langle\phi\left(x_{1}\right) \ldots \phi\left(x_{n}\right)\right\rangle_{\text {conexos }} .
$$

Agora que determinamos o comportamento de $W[J]$, iremos utilizá-lo para obter um outro funcional, que como veremos, irá gerar as funções de correlação próprias. Para obter esse novo funcional, consideraremos a seguinte transformação de Legendre,

$$
\Gamma\left[\phi_{c}\right]=W[J]-\int d^{4} x J(x) \phi_{c}(x),
$$

em que $\phi_{c}$ é um campo conjugado à corrente $J$ através da relação:

$$
\frac{\delta W[J]}{\delta J(x)}=\phi_{c}(x)
$$

É importante notar que $\phi_{c}$ é igual a média do campo $\phi$, porém, diferentemente do ocorrido para (A.7), agora estamos considerando os efeitos da fonte externa.

E como o funcional $\Gamma$ depende de $\phi_{c}$, calcularemos a variação de (A.12) em relação a esse campo

$$
\begin{aligned}
\frac{\delta \Gamma\left[\phi_{c}\right]}{\delta \phi_{c}(x)} & =\frac{\delta W[J]}{\delta \phi_{c}(x)}-\int d^{4} x^{\prime} \frac{\delta J\left(x^{\prime}\right)}{\delta \phi_{c}(x)} \phi_{c}\left(x^{\prime}\right)-J(x) \\
& =\int d^{4} x^{\prime} \frac{\delta W[J]}{\delta J\left(x^{\prime}\right)} \frac{\delta J\left(x^{\prime}\right)}{\delta \phi_{c}(x)}-\int d^{4} x^{\prime} \frac{\delta J\left(x^{\prime}\right)}{\delta \phi_{c}(x)} \frac{\delta W[J]}{\delta J\left(x^{\prime}\right)}-J(x) \\
& =-J(x) .
\end{aligned}
$$

Na dedução acima, utilizamos, na passagem da primeira para a segunda linha, a regra da cadeia no primeira termo, e substituímos (A.13) no segundo termo. Adicionalmente, o resultado acima, define a fonte externa $J$ como um funcional do campo $\phi_{c}$, e têm a mesma estrutura das equações de Euler-Lagrange para um sistema sob os efeitos de uma fonte externa,

$$
\frac{\delta S[\phi]}{\delta \phi(x)}=-J(x)
$$

o que origina a denominação ação efetiva para $\Gamma$.

Posto isto, com o intuito de estabelecer relações entre a ação efetiva e as funções de correlação conexas, iniciaremos notando que

$$
\frac{\delta^{2} \Gamma\left[\phi_{c}\right]}{\delta J(y) \delta \phi_{c}(x)}=-\delta(x-y)
$$

Ao aplicar a regra da cadeia para a derivação funcional em relação à corrente,

$$
\frac{\delta}{\delta J(y)}=\int d^{4} z \frac{\delta \phi_{c}(z)}{\delta J(y)} \frac{\delta}{\delta \phi_{c}(z)}=\int d^{4} z \frac{\delta^{2} W[J]}{\delta J(y) \delta J(z)} \frac{\delta}{\delta \phi_{c}(z)},
$$


no lado esquerda de (A.16), temos

$$
\begin{array}{r}
-\int d^{4} z \frac{\delta \phi_{c}(z)}{\delta J(y)} \frac{\delta^{2} \Gamma\left[\phi_{c}\right]}{\delta \phi_{c}(z) \delta \phi_{c}(x)}=\delta(x-y) \\
-\int d^{4} z \frac{\delta^{2} W[J]}{\delta J(y) \delta J(z)} \frac{\delta^{2} \Gamma\left[\phi_{c}\right]}{\delta \phi_{c}(z) \delta \phi_{c}(x)}=\delta(x-y) .
\end{array}
$$

Esse resultado estabelece que $\frac{\delta^{2} \Gamma\left[\phi_{c}\right]}{\delta \phi_{c} \delta \phi_{c}}$ é a função inversa de $-\frac{\delta^{2} W[J]}{\delta J \delta J}$, que por sua vez é igual a $i$ vezes o propagador conexo, conforme (A.9), então a segunda derivação funcional da ação efetiva é igual a $-i$ vezes o inverso do propagador conexo.

E como o inverso do propagador, conforme (B.7), está relacionado diretamente com as funções de n pontos, e estas são geradas pelos diagramas 1PI, então a expressão (A.18) indica que $\Gamma$ é o funcional gerador das funções de correlação próprias.

Para nos convencer de que este tipo de relação perdura para todas as ordens superiores, derivaremos a expressão (A.18) em função da corrente,

$$
\int d^{4} z \frac{\delta^{3} W[J]}{\delta J(u) \delta J(y) \delta J(z)} \frac{\delta^{2} \Gamma\left[\phi_{c}\right]}{\delta \phi_{c}(z) \phi_{c}(x)}+\int d^{4} z \frac{\delta^{2} W[J]}{\delta J(y) \delta J(z)} \frac{\delta^{3} \Gamma\left[\phi_{c}\right]}{\delta J(u) \delta \phi_{c}(z) \phi_{c}(x)}=0 .
$$

Usando novamente a regra da cadeia, (A.17), no segundo termo da equação acima, obtemos

$\int d^{4} z \frac{\delta^{3} W[J]}{\delta J(u) \delta J(y) \delta J(z)} \frac{\delta^{2} \Gamma\left[\phi_{c}\right]}{\delta \phi_{c}(z) \phi_{c}(x)}=-\int d^{4} z d^{4} w \frac{\delta^{2} W[J]}{\delta J(y) \delta J(z)} \frac{\delta^{3} \Gamma\left[\phi_{c}\right]}{\delta \phi_{c}(z) \delta \phi_{c}(x) \phi_{c}(w)} \frac{\delta^{2} W[J]}{\delta J(w) \delta J(u)}$.

Recorrendo à expressão (A.18), podemos reescrever a equação acima como

$$
\frac{\delta^{3} W[J]}{\delta J\left(x_{1}\right) \delta J\left(x_{2}\right) \delta J\left(x_{3}\right)}=\int d^{4} y_{1} d^{4} y_{2} d^{4} y_{3} \frac{\delta^{2} W[J]}{\delta J\left(x_{1}\right) \delta J\left(y_{1}\right)} \frac{\delta^{2} W[J]}{\delta J\left(x_{2}\right) \delta J\left(y_{2}\right)} \frac{\delta^{2} W[J]}{\delta J\left(x_{3}\right) \delta J\left(y_{3}\right)} \times
$$

Esse resultado nos mostra, que a função de correlação conexa pode ser decomposta em uma estrutura, na qual temos como base a função de correlação própria, e a cada perna externa associamos um propagador conexo. Para o caso das funções de correlação de 3 pontos, podemos representar diagramaticamente essa conexão como

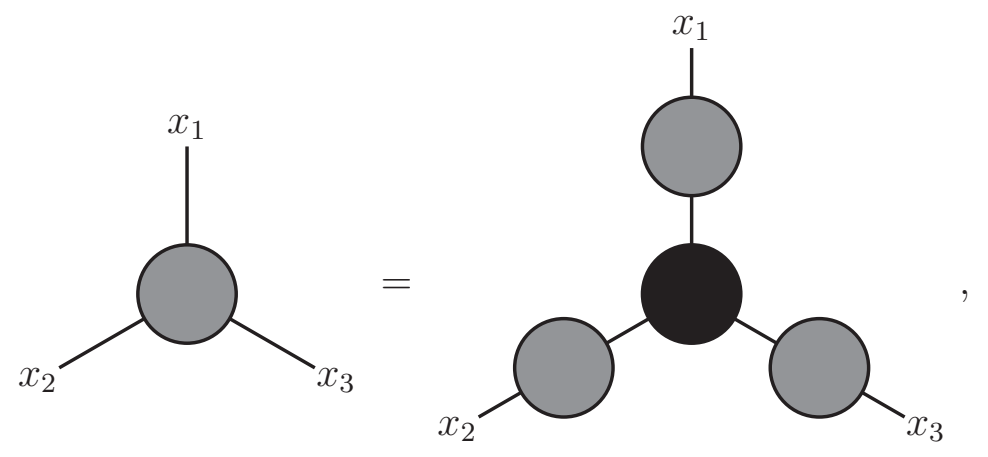


em que os círculos cinzas representam a soma dos diagramas conexos, e o círculo preto representa a soma dos diagramas 1 PI.

Esse tipo de conexão é válida para todas as próximas ordens, e assim podemos escrever a função de correlação própria, П, para qualquer ordem $n$, como

$$
\Pi\left(x_{1}, \ldots, x_{n}\right)=\left.i \frac{\delta^{n} \Gamma[\phi]}{\delta \phi\left(x_{1}\right) \ldots \delta \phi\left(x_{n}\right)}\right|_{\phi=0} .
$$

De forma equivalente, é possível utilizar (A.14), e escrever a equação acima em função da corrente externa,

$$
\Pi\left(x_{1}, \ldots, x_{n}\right)=-\left.i \frac{\delta^{n-1} J\left(x_{n}\right)}{\delta \phi\left(x_{1}\right) \ldots \delta \phi\left(x_{n-1}\right)}\right|_{\phi=0} .
$$

Até o presente momento, consideramos que a ação efetiva é um funcional diferenciável, e portanto, será também expansível em uma série de Taylor,

$$
\Gamma[\phi]=\left.\sum_{n=1}^{\infty} \frac{1}{n !} \int d^{4} x_{1} \ldots d^{4} x_{n} \frac{\delta^{n} \Gamma[\phi]}{\delta \phi\left(x_{1}\right) \ldots \delta \phi\left(x_{n}\right)}\right|_{\phi=0} \phi\left(x_{1}\right) \ldots \phi\left(x_{n}\right) .
$$

Por fim, ao substituir (A.24) nesta expansão, obteremos

$$
\Gamma[\phi]=-i \sum_{n=1}^{\infty} \frac{1}{n !} \int d^{4} x_{1} \ldots d^{4} x_{n} \Pi\left(x_{1}, \ldots, x_{n}\right) \phi\left(x_{1}\right) \ldots \phi\left(x_{n}\right) .
$$

\section{A.1 Funcional gerador das funções de correlação}

Assim como fizemos em (2.27), vamos aqui admitir que a exponencial da parte de interação da ação em (A.1) é passível de uma expansão em série de potências. Desta forma, podemos reescrever a expressão para o funcional gerador das funções de correlação como

$$
\begin{aligned}
Z[J] & =\int[d \phi] e^{i \int d^{4} x\left[\mathcal{L}_{0}-V[\phi]+J(x) \phi(x)\right]} \\
& =\int[d \phi] \sum_{n=0}^{\infty} \frac{S_{I}^{n}}{n !} e^{i \int d^{4} x\left[\mathcal{L}_{0}+J(x) \phi(x)\right]}
\end{aligned}
$$

Nesta expressão, estamos considerando que a parte de interação é totalmente representada por um potencial $V$, e este por sua vez admite uma expansão em série de potências do campo $\phi$.

$$
\begin{aligned}
Z[J] & =\int[d \phi] \sum_{n=0}^{\infty}(-i)^{n} \int d^{4} x_{1} \ldots d^{4} x_{n} V\left[\phi\left(x_{1}\right)\right] \ldots V\left[\phi\left(x_{n}\right)\right] e^{i \int d^{4} x\left[\mathcal{L}_{0}+J(x) \phi(x)\right]} \\
& =\int[d \phi] \sum_{n=0}^{\infty}(-i)^{n} \int d^{4} x_{1} \ldots d^{4} x_{n} \sum_{l_{1}=0}^{\infty} V_{l_{1}} \phi^{l_{1}}\left(x_{1}\right) \ldots \sum_{l_{n}=0}^{\infty} V_{l_{n}} \phi^{l_{n}}\left(x_{n}\right) e^{i \int d^{4} x\left[\mathcal{L}_{0}+J(x) \phi(x)\right]}
\end{aligned}
$$


Como vimos em (A.2), para cada um desses campos, provenientes das expansões de $V$, podemos efetuar a substituição $\phi\left(x_{j}\right) \rightarrow-i \frac{\delta}{\delta J\left(x_{j}\right)}$, culminando em

$$
Z[J]=\int[d \phi] e^{V\left(-\frac{\delta}{\delta J}\right)} e^{i \int d^{4} x\left[\mathcal{L}_{0}+J(x) \phi(x)\right]} .
$$

Agora, para lidar com o termo restante na expressão acima, primeiramente, consideraremos que a parte cinética da ação deste funcional gerador pode ser escrita como

$$
S_{0}=\int d^{4} x \frac{1}{2} \phi(x)\left(-\partial^{2}-m^{2}\right) \phi(x),
$$

e adicionalmente, definiremos

$$
W_{0}[J]=\int d^{4} x\left[\frac{1}{2} \phi(x)\left(-\partial^{2}-m^{2}\right) \phi(x)+J(x) \phi(x)\right] .
$$

A fim de desacoplar o campo $\phi$ da corrente externa, aplicaremos nesta relação, a seguinte mudança de variável $\phi(x)=\phi^{\prime}(x)+i \int d^{4} y D(x-y) J(y)$, cujo Jacobiano é unitário, e em que $D(x-y)$ é a função de Green no espaço das posições.

$$
\begin{array}{r}
W_{0}[J]=\int d^{4} x\left[\frac{1}{2} \phi^{\prime}(x)\left(-\partial^{2}-m^{2}\right) \phi^{\prime}(x)+\frac{i}{2} \int d^{4} z \phi^{\prime}(x)\left(-\partial^{2}-m^{2}\right) D(x-z) J(z)+\right. \\
+\frac{i}{2} \int d^{4} y D(x-y) J(y)\left(-\partial^{2}-m^{2}\right) \phi^{\prime}(x)- \\
-\frac{1}{2} \int d^{4} y d^{4} z D(x-y) J(y)\left(-\partial^{2}-m^{2}\right) D(x-z) J(z)+ \\
\left.+i \int d^{4} J(x) D(x-y) J(y)+J(x) \phi^{\prime}(x)\right]
\end{array}
$$

Após alguns cancelamentos, que surgem ao substituirmos a equação de movimento $\left(-\partial^{2}-m^{2}\right) D\left(x-x^{\prime}\right)=i \delta\left(x-x^{\prime}\right)$ em (A.32), temos

$$
W_{0}[J]=\int d^{4} x\left[\frac{1}{2} \phi^{\prime}(x)\left(-\partial^{2}-m^{2}\right) \phi^{\prime}(x)+\frac{i}{2} \int d^{4} y J(x) D(x-y) J(y)\right] .
$$

Retornando esse resultado em (A.29), obteremos uma expressão para o funcional gerador $Z$ que é explicitamente independente do campo $\phi$.

$$
Z[J]=Z[0] e^{V\left(-\frac{\delta}{\delta J}\right)} e^{-\frac{1}{2} \int d^{4} x d^{4} y J(x) D(x-y) J(y)}
$$

Além disso, esse formato de $Z$ é mais conveniente que o expresso em (A.1), a depender da aplicação. 


\section{APÊNDICE B - Propagadores no ensemble completo}

O presente apêndice, em contraste ao que ocorreu nos primeiros capítulos, nos quais utilizamos apenas os propagadores livres, tem o intuito de analisar a forma dos propagadores quando considerada a teoria completa. Sendo assim, iniciaremos pela definição do propagador à temperatura finita no espaço das configurações introduzida no apêndice $\mathrm{A}$,

$$
D(x, y)=\langle\mathcal{T} \phi(x) \phi(y)\rangle
$$

Por questões de conveniência, substituiremos as transformadas de Fourier dos campos $\phi$ na expressão acima, e fixaremos $y=0$.

$$
D(x, y)=\frac{\beta}{V} \sum_{l, j} \int d^{3} p \int d^{3} q e^{i \vec{p} \cdot \vec{x}} e^{i \omega_{l} \cdot \tau}\left\langle\tilde{\phi}_{l}(\vec{p}) \tilde{\phi}_{j}(\vec{q})\right\rangle
$$

A fim de extrair da expressão acima o propagador definido no espaço dos momentos, multiplicaremos esta equação por $e^{-i\left(\vec{k} \cdot \vec{x}+\omega_{n} \cdot \tau\right)}$, e integraremos sob todo o espaço euclidiano $x_{E}=(\tau, \vec{x})$.

$$
\begin{aligned}
\int_{0}^{\beta} d \tau \int d^{3} x e^{-i\left(\vec{k} \cdot \vec{x}+\omega_{n} \cdot \tau\right)} D(x, y) & =\frac{\beta}{V} \sum_{l, j} \int d^{3} p d^{3} q \int d^{4} x_{E} e^{i\left[(\vec{p}-\vec{k}) \cdot \vec{x}+\left(\omega_{l}-\omega_{n}\right) \cdot \tau\right]}\left\langle\tilde{\phi}_{l}(\vec{p}) \tilde{\phi}_{j}(\vec{q})\right\rangle \\
D\left(\omega_{n}, \vec{k}\right) & =\beta^{2}\left\langle\tilde{\phi}_{n}(\vec{k}) \tilde{\phi}_{-n}(-\vec{k})\right\rangle
\end{aligned}
$$

Na expressão acima, para que a integral no lado direito da primeira linha não fosse nula, as seguintes condições deveriam ser satisfeitas, $j=-l$ e $\vec{q}=-\vec{k}$, o que culminou na segunda linha. Essas restrições são geradas pelas simetrias das somas em $j$ e $l$, e das integrais em $q$ e $p$.

Essa média, em (B.3), que contempla o produto dos campos $\phi$, diferentemente dos casos anteriores, será efetuada no ensemble completo, isto é, iremos considerar todas as interações, então,

$$
D\left(\omega_{n}, \vec{k}\right)=\beta^{2} \frac{\int d[\phi] e^{S} \tilde{\phi}_{n}(\vec{k}) \tilde{\phi}_{-n}(-\vec{k})}{\int d[\phi] e^{S}} .
$$

Além disso, essa ação $S$ expressa acima, contém duas partes, uma quadrática no campo $\phi$, e outra contendo os termos de interação, que será identificada como $S_{I}$. Portanto, 
podemos escrever, de forma genérica,

$$
S=-\frac{1}{2} \tilde{\phi}_{n}(\vec{k}) D_{0}^{-1} \tilde{\phi}_{-n}(-\vec{k})+S_{I},
$$

em que $D_{0}$ é o já definido propagador livre. Dito isto, não é difícil notar que uma derivação funcional de $\int[d \phi] e^{S}$ em relação a $D_{0}^{-1}$, reproduz (B.3). Desta forma, temos

$$
\begin{aligned}
D\left(\omega_{n}, \vec{k}\right) & =-2 \frac{\delta \ln (Z)}{\delta D_{0}^{-1}}=-2 \frac{\delta D_{0}^{-1}}{\delta D_{0}} \frac{\delta \ln (Z)}{\delta D_{0}} \\
& =2 D_{0}^{2} \frac{\delta \ln (Z)}{\delta D_{0}} .
\end{aligned}
$$

Esse resultado sugere que $D$ depende diretamente de $D_{0}$, sendo assim, utilizaremos o seguinte ansatz:

$$
\begin{aligned}
D\left(\omega_{n}, \vec{k}\right) & =\frac{1}{\omega_{n}^{2}+\vec{k}^{2}+m^{2}+\Pi\left(\omega_{n}, \vec{k}\right)} \\
& =\frac{D_{0}}{1+D_{0} \Pi} .
\end{aligned}
$$

Essa função $\Pi\left(\omega_{n}, \vec{k}\right)$, que agora simplesmente representa a diferença entre o propagador livre e o da teoria completa, possui uma importante interpretação física, que será demonstrada no decorrer desta seção, sendo que em casos gerais, ela pode ou não depender da energia e dos momentos dos campos, e em certos limites corresponderá à uma massa efetiva, como pode ser visto no capítulo 4. Ademais, a forma da equação (B.7) permite interpretar, que a função $\Pi$ irá contribuir com correções para o propagador livre. Para evidenciar este fato, basta expandir (B.7) em relação à $D_{0} \Pi$.

$$
D\left(\omega_{n}, \vec{k}\right)=D_{0}\left[1-D_{0} \Pi+\left(D_{0} \Pi\right)^{2}-\left(D_{0} \Pi\right)^{3}+\ldots\right]
$$

Dando prosseguimento, ao substituirmos (B.7) em (B.6), temos

$$
\begin{aligned}
\frac{D_{0}}{1+D_{0} \Pi} & =2 D_{0}^{2} \frac{\delta\left(\ln \left(Z_{0}\right)+\ln \left(Z_{I}\right)\right)}{\delta D_{0}} \\
\frac{1}{1+D_{0} \Pi} & =2 D_{0}\left(\frac{\delta \ln \left(Z_{0}\right)}{\delta D_{0}}+\frac{\delta \ln \left(Z_{I}\right)}{\delta D_{0}}\right) .
\end{aligned}
$$

Esse logaritmo da função de partição livre pode ser facilmente obtido se regressarmos a expressão

$$
Z_{0}=N^{\prime} \prod_{n, k} \int_{-\infty}^{\infty} d \phi_{n}(\vec{k}) e^{-\frac{1}{2} \phi_{n}(\vec{k}) \beta^{2}\left(\omega_{n}^{2}+\vec{k}^{2}+m^{2}\right) \phi_{n}(\vec{k})}
$$

Como a integral em $\phi$ é gaussiana, ela resulta em

$$
Z_{0}=N^{\prime} \prod_{n, k} \frac{1}{\sqrt{\beta^{2} D_{0}^{-1}\left(\omega_{n}, \vec{k}\right)}} .
$$


Agora, basta tomarmos o logaritmo dos dois lados desta equação,

$$
\ln \left(Z_{0}\right)=\frac{1}{2} \sum_{n} \sum_{k} \ln \left[\frac{D_{0}\left(\omega_{n}, \vec{k}\right)}{\beta^{2}}\right]+\ln \left(N^{\prime}\right) .
$$

E assim concluímos, que o primeiro termo a direita da equação (B.9) é simplesmente

$$
\frac{\delta \ln \left(Z_{0}\right)}{\delta D_{0}}=\frac{1}{2 D_{0}}
$$

Ao regressarmos esse resultado na equação (B.9), podemos reescrevê-la como

$$
\frac{1}{1+D_{0} \Pi}=1+2 D_{0} \frac{\delta \ln \left(Z_{I}\right)}{\delta D_{0}} \text {. }
$$

A partir desta relação é possível calcular qualquer ordem das correções dos propagadores. Adicionalmente, para que identifiquemos com qual das ordens de aproximação estamos lidando, faremos uma expansão em $\Pi$ de acordo com sua potência $l$ na constante de acoplamento,

$$
\Pi=\sum_{l=1}^{\infty} \Pi_{l}
$$

Com o intuito de exemplificar a mecânica envolvida na utilização de (B.14), calcularemos $\Pi_{1}$ para a teoria $\phi^{4}$. Utilizando a expansão (B.8) até o segundo termo, temos

$$
\begin{aligned}
1-D_{0} \Pi_{1} & =1+2 D_{0} \frac{\delta \ln \left(Z_{1}\right)}{\delta D_{0}} \\
\Pi_{1} & =-2 \frac{\delta \ln \left(Z_{1}\right)}{\delta D_{0}} .
\end{aligned}
$$

A substituição da expressão (2.37) de $\ln \left(Z_{1}\right)$ na equação acima, culminará em

$$
\begin{aligned}
\Pi_{1} & =-2 \frac{\delta}{\delta D_{0}}\left[-3 \lambda \beta V\left(T \sum_{n_{1}} \int \frac{d^{3} p_{1}}{(2 \pi)^{3}} D_{0}\left(\omega_{n_{1}}, \vec{p}_{1}\right)\right)\left(T \sum_{n_{2}} \int \frac{d^{3} p_{2}}{(2 \pi)^{3}} D_{0}\left(\omega_{n_{2}}, \vec{p}_{2}\right)\right)\right] \\
& =6 \lambda \beta V\left[\left(T \sum_{n_{1}} \int \frac{d^{3} p_{1}}{(2 \pi)^{3}} \beta(2 \pi)^{3} \delta^{(4)}\left(x-x_{1}\right)\right)\left(T \sum_{n_{2}} \int \frac{d^{3} p_{2}}{(2 \pi)^{3}} D_{0}\left(\omega_{n_{2}}, \vec{p}_{2}\right)\right)+1 \leftrightarrow 2\right] \\
& =-12
\end{aligned}
$$

Esse resultado é muito útil, pois permite visualizar qual é a ação do operador $\frac{\delta}{\delta D_{0}}$ em um diagrama de Feynman. Para tornar isto mais explícito, reescreveremos a equação (B.16) em sua forma diagramática:

$$
\Pi_{1}=-2 \frac{\delta}{\delta D_{0}}(3 \bigcirc)=-12 \bigcirc
$$


Aqui vemos, que a derivação funcional em relação à $D_{0}$ é equivalente a cortarmos uma das linhas do diagrama. Além disso, este corte deve ocorrer em todas as linhas internas, o que gerou um fator 2 no resultado acima.

A fim de compreender esse mecanismo mais profundamente, seguiremos para a próxima ordem de aproximação, $l=2$.

$$
\begin{aligned}
-D_{0} \Pi_{2}+\left(D_{0} \Pi_{1}\right)\left(D_{0} \Pi_{1}\right) & =2 D_{0} \frac{\delta \ln \left(Z_{2}\right)}{\delta D_{0}} \\
\Pi_{2} & =-2 \frac{\delta \ln \left(Z_{2}\right)}{\delta D_{0}}+\Pi_{1} D_{0} \Pi_{1} \\
\Pi_{2} & =-2 \frac{\delta \ln \left(Z_{2}\right)}{\delta D_{0}}+144
\end{aligned}
$$

Novamente recorrendo aos resultados do primeiro capítulo, temos

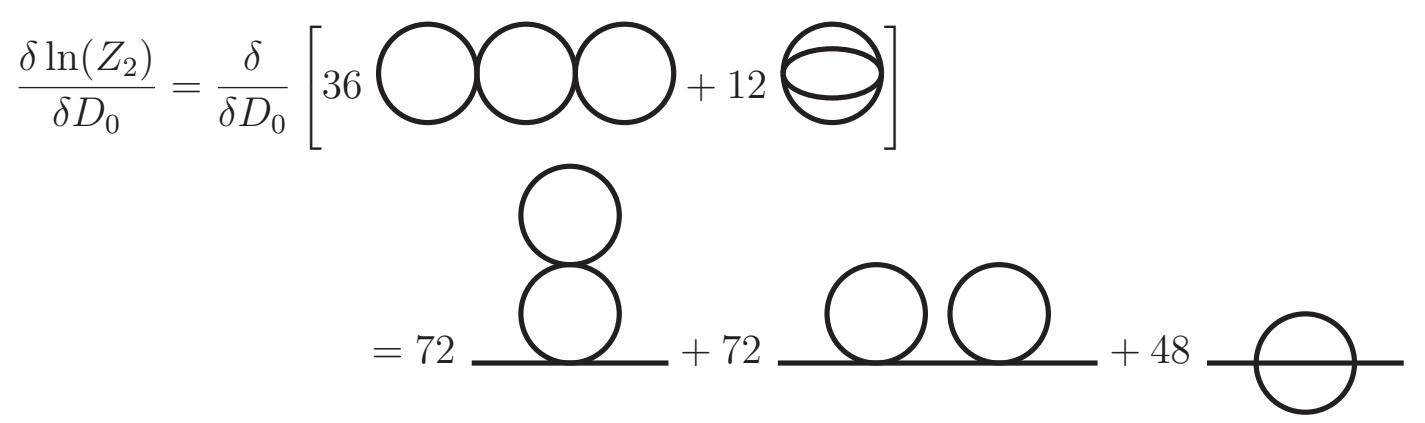

Retornando esse resultado em (B.19),

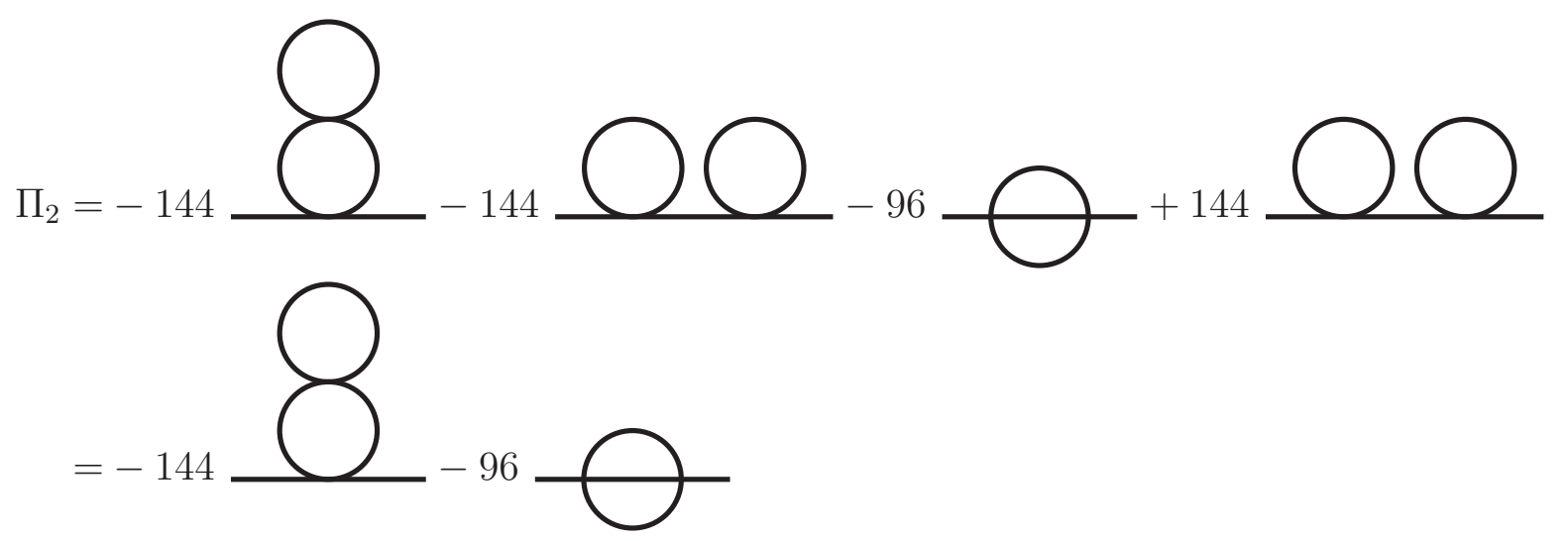

Após o cancelamento acima, é notável que os termos sobreviventes são do tipo 1PI, que de forma resumida, podem ser definidos como aqueles diagramas que ao sofrerem um corte em uma de suas linhas/propagadores, não irão gerar dois outros diagramas desconectados. 
Esse comportamento irá se repetir para todas as próximas ordens de aproximação, o que nos leva ao seguinte procedimento para a obtenção de $\Pi_{l}$ : primeiramente, construiremos todos os diagramas, com a ordem $l$ desejada, que contribuem para $\ln \left(Z_{I}\right)$; em seguida aplicaremos o operador $-2 \frac{\delta}{\delta D_{0}}$ nesses diagramas; e por fim, coletaremos apenas os diagramas 1PI.

$$
\Pi_{l}=\left[-2\left(\frac{\delta \ln \left(Z_{I}\right)}{\delta D_{0}}\right)_{l}\right]_{1 \mathrm{PI}}
$$





\section{APÊNDICE C - Integrais angulares}

Iniciaremos os cálculos desse apêndice pela seguinte integral:

$$
I=\int d \Omega_{D-1}\left(p_{j} \frac{\partial}{\partial p_{i}}-p_{i} \frac{\partial}{\partial p_{j}}\right) \frac{p_{j} k_{i}}{k_{0}|\vec{p}|-\vec{k} \vec{p}} .
$$

Ao tratar, isoladamente, cada um dos integrandos acima, temos

$$
\begin{gathered}
p_{j} \frac{\partial}{\partial p_{i}}\left(\frac{p_{j} k_{i}}{k_{0} \cdot|\vec{p}|-\vec{k} \cdot \vec{p}}\right)=\frac{p_{j} \delta_{i j} k_{i}}{k_{0} \cdot|\vec{p}|-\vec{k} \cdot \vec{p}}+\frac{p_{j} p_{j} k_{i} \delta_{i l} k_{l}}{\left(k_{0} \cdot|\vec{p}|-\vec{k} \cdot \vec{p}\right)^{2}}=\frac{\vec{k} \cdot \vec{p}}{k \cdot p}+\frac{\vec{k}^{2} \vec{p}^{2}}{(k \cdot p)^{2}}, \\
p_{i} \frac{\partial}{\partial p_{j}}\left(\frac{p_{j} k_{i}}{k_{0} \cdot|\vec{p}|-\vec{k} \cdot \vec{p}}\right)=\frac{p_{i} \delta_{j j} k_{i}}{k_{0} \cdot|\vec{p}|-\vec{k} \cdot \vec{p}}+\frac{p_{i} p_{j} k_{i} \delta_{j l} k_{l}}{\left(k_{0} \cdot|\vec{p}|-\vec{k} \cdot \vec{p}\right)^{2}}=\frac{(D-1) \vec{k} \cdot \vec{p}}{k \cdot p}+\frac{(\vec{k} \cdot \vec{p})^{2}}{(k \cdot p)^{2}} .
\end{gathered}
$$

Assim, retornando esses termos em (C.1), culminamos com

$$
\begin{aligned}
I & =\int d \Omega_{D-1}\left[\frac{\vec{k} \cdot \vec{p}}{k \cdot p}+\frac{\vec{k}^{2} \vec{p}^{2}}{(k \cdot p)^{2}}-\frac{(D-1) \vec{k} \cdot \vec{p}}{k \cdot p}-\frac{(\vec{k} \cdot \vec{p})^{2}}{(k \cdot p)^{2}}\right] \\
& =\int d \Omega_{D-1} \frac{-(D-2) \vec{k} \cdot \vec{p} k_{0} p_{0}+(D-3)(\vec{k} \cdot \vec{p})^{2}+\vec{k}^{2} \vec{p}^{2}}{(k \cdot p)^{2}} .
\end{aligned}
$$

$\mathrm{E} \operatorname{como}(\vec{k} \cdot \vec{p})^{2}=(k \cdot p)^{2}-\left(k_{0} p_{0}\right)^{2}+2 \vec{k} \cdot \vec{p} k_{0} p_{0}$, então

$$
\begin{aligned}
I & =\int d \Omega_{D-1}\left[\frac{(D-4) \vec{k} \cdot \vec{p} k_{0} p_{0}-(D-3)\left(k_{0} p_{0}\right)^{2}+\vec{k}^{2} \vec{p}^{2}}{(k \cdot p)^{2}}+(D-3)\right] \\
& =\int d \Omega_{D-1}\left[\frac{-(D-4) k_{0} p_{0}(k \cdot p)-k^{2} p_{0}^{2}}{(k \cdot p)^{2}}+(D-3)\right] \\
& =\int d \Omega_{D-1}\left[\frac{-(D-4) k_{0} p_{0}}{k \cdot p}+(D-3)\right]-\int d \Omega_{D-1} \frac{k^{2} p_{0}^{2}}{(k \cdot p)^{2}} .
\end{aligned}
$$

Regressando novamente à (C.1), e observando que a medida de integração $d \Omega_{D-1}$ pode ser reescrita como $d p_{1} \ldots d p_{D-1} \delta\left(p_{1}^{2}+p_{2}^{2}+\ldots+p_{D-1}^{2}-1\right)$, obteremos

$$
I=\int d p_{1} \ldots d p_{D-1} \delta\left(p_{1}^{2}+\ldots+p_{D-1}^{2}-1\right)\left(p_{j} \frac{\partial}{\partial p_{i}}-p_{i} \frac{\partial}{\partial p_{j}}\right) \frac{p_{j} k_{i}}{k_{0}|\vec{p}|-\vec{k} \cdot \vec{p}} .
$$

Por fim, uma integração por partes na integral acima evidencia que este termo é nulo, e portanto

$$
\int d \Omega_{D-1} \frac{k^{2} p_{0}^{2}}{(k \cdot p)^{2}}=\int d \Omega_{D-1}\left[\frac{-(D-4) k_{0} p_{0}}{k \cdot p}+(D-3)\right] .
$$





\section{APÊNDICE D - Função térmica no limite de massa nula}

No limite de massa nula, vimos que toda a dependência na temperatura pode ser expressa pela equação 6.16, de modo que todas as amplitudes térmicas possuem um fator $T^{4-n} I^{(n)}(\bar{m})$. Definindo $R^{(n)}(\bar{m}) \equiv \bar{m}^{n-4} I^{(n)}(\bar{m})$, podemos fazer uma análise gráfica.

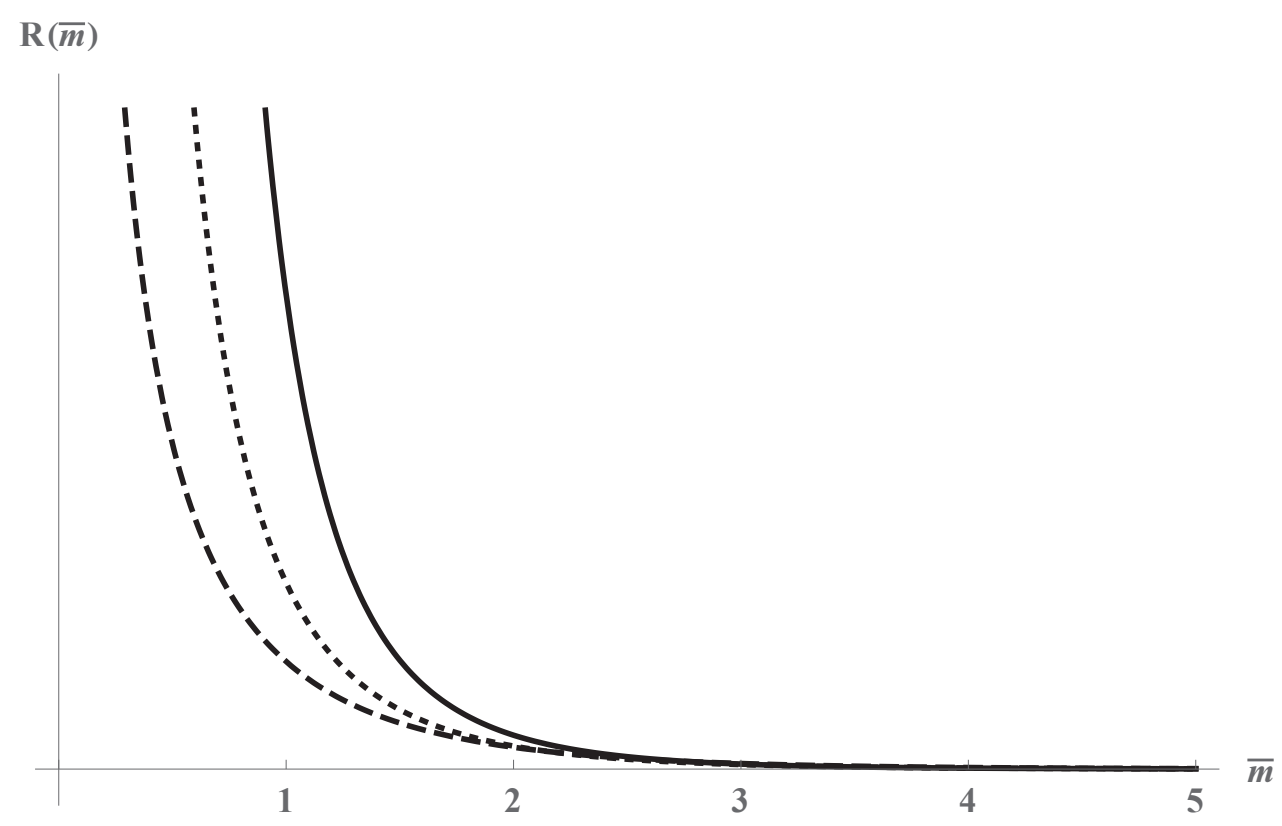

Figura 17 - Dependência das amplitudes térmicas em relação ao parâmetro $\bar{m}=\frac{m}{T}$, para $n=1,2$ e 3 .

No gráfico 17, observa-se para $n=1,2$ e 3, um comportamento semelhante. Quando o fator $\bar{m}$ tende a um número grande, isto é, quando a temperatura é baixa, todas as ordens decrescem, e tendem a zero. Da mesma forma, quando $\bar{m}$ tende a zero, indicando que a temperatura é muito maior que a massa dos férmions, todas as ordens tendem ao infinito. Porém, é notável que para $n=1$, este crescimento inicia-se a uma temperatura menor do que para $n=2$ e $n=3$, indicando ser este o termo dominante. Ademais, uma relação semelhante ocorre entre $n=2$ e $n=3$, e portanto, concluímos que $n=2$ gera o termo subdominante.

Já para $n=4$, figura 18 , atribui-se o crescimento abrupto, na região onde $\bar{m}$ é pequeno, à divergência do termo $R^{(4)}$, que ocorre no limite de massa nula. Esta divergência, na região do infravermelho, também ocorrerá para todas as próximas ordens ímpares, $n \geq 5$. 


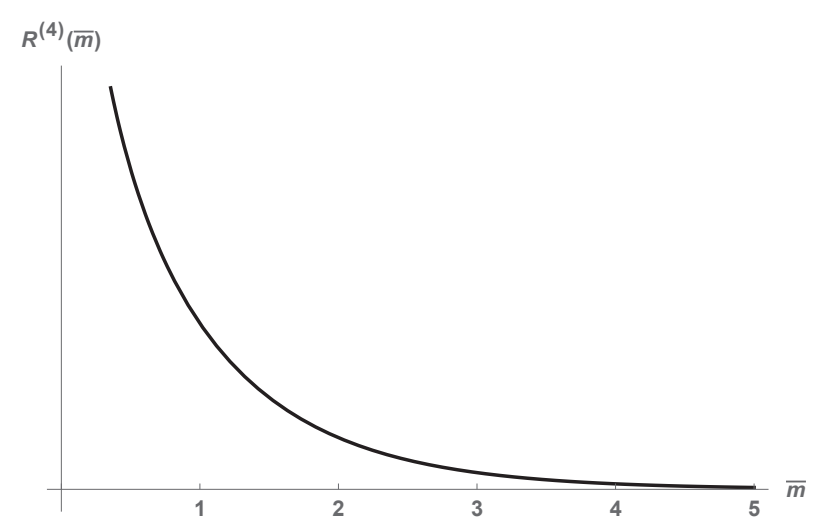

Figura 18 - Dependência das amplitudes térmicas em relação ao parâmetro $\bar{m}=\frac{m}{T}$, para $n=4$.

A fim de estudar essas divergências analiticamente, analisaremos

$$
\mathcal{I}_{n}=\int_{0}^{\infty} d|\vec{k}| \frac{|\vec{k}|^{3-n}}{e^{\beta|\vec{k}|}+1}
$$

Esta integral representa a parte radial das amplitudes térmicas, e para as três primeiras ordens de aproximação, temos

$$
\begin{gathered}
\mathcal{I}_{1}=\frac{3 T^{3}}{2} \zeta(3) \\
\mathcal{I}_{2}=\frac{\pi^{2} T^{2}}{12} \\
\mathcal{I}_{3}=T \ln (2)
\end{gathered}
$$

Aqui vemos, que $\mathcal{I}_{n}$ é finita para essas ordens mais baixas de n, e além do mais fica claro que o primeiro termo é o dominante, e o segundo é o subdominante. Porém, a partir de $n=4$, será necessário regularizar a integral em (D.1).

$$
\mathcal{I}_{n}^{r d}=\left(\frac{e^{\gamma}}{\mu}\right)^{\epsilon} \int_{0}^{\infty} d|\vec{k}| \frac{|\vec{k}|^{\epsilon+3-n}}{e^{\beta|\vec{k}|}+1}
$$

A prescrição escolhida acima, foi a regularização dimensional, onde consideramos o espaço-tempo assumindo uma dimensão igual a $4+\epsilon, \operatorname{com} \epsilon \rightarrow 0$.

Além disto, $\mu$ é um parâmetro com dimensão de massa, que permitirá recuperar as dimensões físicas originais(BRANDT et al., 2002; BRANDT; DAS; FRENKEL, 2003), e $\gamma$ é a constante de Euler-Mascheroni. 
Utilizando novamente $u T \equiv|\vec{k}|$, obteremos

$$
\begin{aligned}
\mathcal{I}_{n}^{r d} & =T^{4-n}\left(\frac{e^{\gamma} T}{\mu}\right)^{\epsilon} \int_{0}^{\infty} d u \frac{u^{\epsilon+3-n}}{e^{u}+1} \\
& =T^{4-n}\left(\frac{e^{\gamma} T}{\mu}\right)^{\epsilon}\left(1-\frac{1}{2^{3-n+\epsilon}}\right) \Gamma(4-n+\epsilon) \zeta(4-n+\epsilon)
\end{aligned}
$$

Nesta expressão, para $n \leq 3$, é possível impor a condição $\epsilon \rightarrow 0$, o que reproduz (D.2a), (D.2b) e (D.2c). No entanto, quando $n \geq 4$, ocorrem polos do tipo $\frac{1}{\epsilon}$ na função $\Gamma(4-n+\epsilon)$, e portanto, deveremos efetuar os cálculos caso a caso.

Para o primeiro destes casos em que ocorre a divergência infravermelha, $n=4$, utilizaremos as seguintes aproximações:

$$
\begin{gathered}
\Gamma(\epsilon) \approx \frac{1}{\epsilon} \\
\left(\frac{T}{\mu}\right)^{\epsilon}=e^{\epsilon \ln \left(\frac{T}{\mu}\right)} \approx 1+\epsilon \ln \left(\frac{T}{\mu}\right),
\end{gathered}
$$

que quando substituídas em (D.4), resultam em

$$
\mathcal{I}_{4}^{r d}=\frac{1}{2} \ln \left(\frac{\pi T}{2 \mu}\right)+\frac{1}{2 \epsilon} .
$$

Pela primeira vez, encontramos uma ordem na qual a dependência da temperatura é diferente de um simples polinômio. Adicionalmente, podemos relacionar a esta divergência contida em (D.6), o comportamento de $R^{(n)}(\bar{m})$, quando $\bar{m}$ está próximo de zero, conforme gráfico 18. Em contrapartida, assim como ocorre para $n=1,2$ e 3 , na região onde $\bar{m}$ é grande, a função $R^{(4)}(\bar{m})$ descresse rapidamente.

Para a próxima ordem, $n=5$, utilizaremos

$$
\begin{gathered}
\Gamma(-1+\epsilon) \approx-\frac{1}{\epsilon}-1+\gamma \\
\zeta(-1+\epsilon) \approx-\frac{1}{12}+\zeta^{\prime}(-1) \epsilon .
\end{gathered}
$$

A composição dessas duas expansões, com as provenientes de $e^{\epsilon \log \left(\frac{e^{\gamma} T}{\mu}\right)}$ e de $e^{\epsilon \log (2)}$, quando substituída em (D.4), nos permite obter:

$$
\mathcal{I}_{5}^{r d}=-\frac{1}{T} \ln \left(\frac{T}{\mu}\right)-\frac{1}{T}+\frac{1}{T}\left(3 \zeta^{\prime}(-1)+\frac{1}{3} \ln (2)-\frac{1}{4 \epsilon}\right) .
$$

Diferentemente dos termos anteriores, $n<5$, caso consideremos o limite $T \rightarrow \infty$, este termo irá se anular. Esta tendência ocorrerá para todas as ordens subsequentes, porém é importante notar que para termos com $n$ par, $n=2 j,(j=3,4,5, \ldots)$, teremos 
$\zeta(4-2 j+\epsilon) \approx \zeta^{\prime}(4-2 j) \epsilon$, que cancela o polo proveniente da função gama. Portanto, todas as funções de Green com $n>4$ e par serão finitas.

Como estes termos pares, com $n>4$, tendem a zero, quando $\bar{m} \sim 0$, eles apresentam um comportamento diferente das funções $\operatorname{com} n=1,2,3$ e 4, e podem ser desprezados no limite de altas temperaturas.

Embora o comportamento das amplitudes térmicas, na região de altas temperaturas, seja diferente entre ordens distintas, todas elas se assemelham, quando considerado o limite de baixas temperaturas. Tal fato, já era esperado, pois, quando tomado o limite de temperatura nula de qualquer contribuição térmica na ação efetiva, estas devem-se anular. 


\section{APÊNDICE E - Algoritmo do cálculo das amplitudes térmicas}

Os cálculos efetuados nos capítulos 3, 4 e 5 para teorias de calibre, e em especial para a QED no capítulo 6, podem se tornar extremamente longos dependendo da quantidade de pernas externas no caso das amplitudes frontais, ou da quantidade de iterações efetuadas na equação de transporte. Portanto se faz necessário a utilização de recursos computacionais, sendo que no caso do presente trabalho utilizamos o FeynCalc(HSIEH; YEHUDAI, 1992), que é um pacote de computação algébrica, do aplicativo Mathematica, destinado à cálculos em Física de Partículas.

Para ambos os formalismos, clássico e quântico, é possível definir algoritmos genéricos, que em linhas gerais, devem ser independentes da ordem de aproximação. Todavia, eles dependem de cálculos prévios, como por exemplo, a confecção dos diagramas de Feynman das amplitudes frontais, que além de depender da teoria de calibre em si, depende também da ordem de aproximação e do tipo dos loops.

A seguir, apresentaremos, para um plasma de fótons e elétrons/pósitrons regido pelas leis da eletrodinâmica, os algoritmos contendo as funções que serão utilizadas nos cálculos futuros, no âmbito de cada uma das teorias separadamente. Algumas dessas funções, por dependerem fortemente da ordem de aproximação, não poderão ser explicitadas, permitindo somente sua descrição.

Porém, antes de iniciarmos estes cálculos, vamos redefinir alguns comandos, a fim de economizar na notação,

$$
\begin{aligned}
F V[k, \mu] & =\text { FourVector }[k, \mu], \\
D S[k] & =\text { DiracSlash }[k], \\
G[\mu, \nu] & =\text { MetricTensor }[\mu, \nu], \\
C O N[a, b] & =\text { Contract }[\text { FourVector }[a, \lambda] \text { FourVector }[b, \lambda] ;
\end{aligned}
$$

em que cada uma dessas funções representa, respectivamente, um quadrivetor, a contração entre um quadrivetor e uma matriz gama de Dirac, a métrica espacial, e a contração entre dois quadrivetores.

\section{E.1 QED}

Iniciaremos com o algoritmo para o limite de altas temperaturas da QED, cujo input será a expressão contendo as amplitudes frontais, a qual foi obtida a partir das regras 
de Feynman. Posto isto, para tornar mais clara a expansão no limite da HTL, separaremos os numeradores dos denominadores.

Os numeradores, na forma descrita abaixo, seguem um certo padrão, que incluí a presença de $k$, momento interno ao loop, em todos os termos, e também os fatores $a_{i}$, que representam combinações dos momentos externos $p_{i}$.

$$
\begin{gathered}
N U M\left[k \_, p 1 \_, p 2_{\ldots}, p 3 \ldots, \ldots, p n \_, \mu 1 \_, \mu 2 \_, \mu 3 \ldots, \ldots, \mu n \_\right]= \\
\text {Expand }[\text { Contract }[\operatorname{Tr}[(D S[k]+m) . G A[\mu 1] .(D S[k+a 1]+m) \cdot G A[\mu 2] .(D S[k+a 2]+m) . \\
G A D[\mu 3] .(D S[k+a 3]+m) \ldots G A[\mu n] .(D S[k+a n]+m)]]] / .\left\{C O N[k, k] \rightarrow m^{2},\right. \\
\left.C O N[k, p 1] \rightarrow \frac{C O N[k, p 1]}{x}, C O N[k, p 2] \rightarrow \frac{C O N[k, p 2]}{x} \ldots C O N[k, p n] \rightarrow \frac{C O N[k, p n]}{x}\right\},
\end{gathered}
$$

em que $G A\left[\mu_{i}\right]$ representa uma matriz gama com índice $\mu_{i}$; e Expand, Contract e $\operatorname{Tr}$, são funções que, respectivamente, expandem os termos de uma expressão, contraem os índices de Lorentz, e calculam o traço nos índices de Dirac.

O principal objetivo de (E.2) é calcular os traços presentes nos numeradores das amplitudes frontais, pois a quantidade de termos, nos quais esta expressão se decompõe, aumenta expressivamente com a ordem da aproximação. Ademais, como $x$ é inversamente proporcional a $k$, ao substituirmos $k \rightarrow \frac{k}{x}$ e considerarmos $x \rightarrow 0$, obteremos a mesma expressão caso considerássemos $k \rightarrow \infty$, assim impondo o limite HTL.

Ainda a cerca da expressão (E.2), como o loop que estamos utilizando é fermiônico, foi imposto que os momentos internos estão na camada de massa, $k^{2}=m^{2}$.

Já para os denominadores, após considerarmos novamente a condição da camada de massa, temos

$$
D E N\left[k_{-}, p i_{-}\right]=\frac{1}{C O N[k, k]+2 C O N[k, p i]} / . C O N[k, p i] \rightarrow \frac{C O N[k, p i]}{x} \text {. }
$$

A expressão acima, define somente o denominador de um único propagador, porém as amplitudes frontais possuem uma sequência destes fatores, que após combinados serão expandidos em uma série, cujo fator de expansão será $x$.

$$
\begin{aligned}
& D E N A M P\left[k_{\ldots}, p 1_{\ldots}, p 2_{\ldots}, \ldots, p n_{-}\right]= \\
& \operatorname{Expand}[N \text { ormal }[\text { Series }[D E N[k, a 1] . D E N[k, a 2] \ldots D E N[k, a n-1],\{x, 0, n\}]]
\end{aligned}
$$

Ao combinarmos os numeradores com os propagadores, iremos obter uma expressão completa, que depende de várias potências da temperatura e/ou de logaritmos multiplicados 
por potências da temperatura. Tal dependência, conforme demonstrado em 6.2 , deve limitar-se a apenas uma potência da temperatura, desta forma, para auxiliar no descarte destes termos nulos presentes nas amplitudes térmicas, definiremos

$$
\begin{gathered}
A M P 0\left[p 1_{-}, p 2_{-}, p 3_{\ldots}, \ldots, p n_{-}, \mu 1_{-}, \mu 2_{-}, \mu 3 \ldots, \ldots, \mu n_{-}\right]= \\
\frac{1}{2} \operatorname{Expand}[\operatorname{Normal}[\operatorname{Series}[N U M[k, a 1, a 2, a 3, \ldots, \mu 1, \mu 2, \mu 3, \ldots] \\
\operatorname{DENAMP}[k, a 1, a 2, \ldots],\{x, 0, n-2\}]]
\end{gathered}
$$

sendo esta fração de um meio proveniente de (6.40).

Agora, para finalmente completar o limite de altas temperaturas, consideraremos a primeira ordem não nula das amplitudes térmicas, e em seguida faremos $x=1$.

$$
\begin{aligned}
& A M P Q\left[p 1_{\_}, p 2_{\_}, p 3_{\ldots}, \ldots, p n_{\_}, \mu 1_{\_}, \mu 2_{\_}, \mu 3 \ldots, \ldots, \mu n_{-}\right]= \\
& \frac{1}{2} \operatorname{Expand}[\operatorname{Normal}[\operatorname{Series}[N U M[k, a 1, a 2, a 3, \ldots, \mu 1, \mu 2, \mu 3, \ldots] D E N A M P[k, a 1, a 2, \ldots], \\
& \{x, 0, n-1\}]]-A M P 0\left[p 1_{\_}, p 2_{\_}, p 3 \_, \ldots, p n_{\_}, \mu 1 \_, \mu 2 \_, \mu 3 \ldots, \ldots, \mu n_{-}\right] . / x \rightarrow 1
\end{aligned}
$$

Esse resultado representa apenas uma das possíveis amplitudes frontais, portanto, para obter o resultado final, devemos somar todas as permutações cíclicas das amplitudes frontais, e todas as permutações das pernas externas, conforme o formalismo do tempo imaginário.

$$
\begin{aligned}
& A M P T O T Q\left[p 1 \_, p 2 \ldots, p 3 \ldots, \ldots, \mu 1 \_, \mu 2 \_, \mu 3 \ldots, \ldots\right]= \\
& {[A M P Q[p 1, p 2, p 3, \ldots, \mu 1, \mu 2, \mu 3, \ldots]+A M P Q[p 2, p 3, \ldots, p 1, \mu 2, \mu 3, \ldots, \mu 1]} \\
& \quad+A M P Q[p 3, p 4, \ldots, p 1, p 2, \mu 3, \mu 4, \ldots, \mu 1, \mu 2]+\ldots]
\end{aligned}
$$

Todo esse procedimento descrito até agora, pode ser resumido pelo fluxograma na figura 19, onde o input é representado pelas amplitudes frontais, que como já comentado devem ser calculadas previamente, e após todos os procedimentos descritos no algoritmo, culminamos com o limite de altas temperaturas das amplitudes térmicas no output.

Agora que já possuímos um algoritmo genérico, partiremos para sua aplicação nos casos das amplitudes térmicas com 3 e 4 fótons externos. Para fins de economia, iremos omitir alguns passos desnecessários ao entendimento dos cálculos, como por exemplo, o carregamento do pacote FeynCalc.

Iniciando com o caso de 3 pernas externas, consideraremos que os momentos dos dois propagadores constantes nas amplitudes frontais serão $p_{1} \mathrm{e}-p_{3}$, assim obedecendo a 


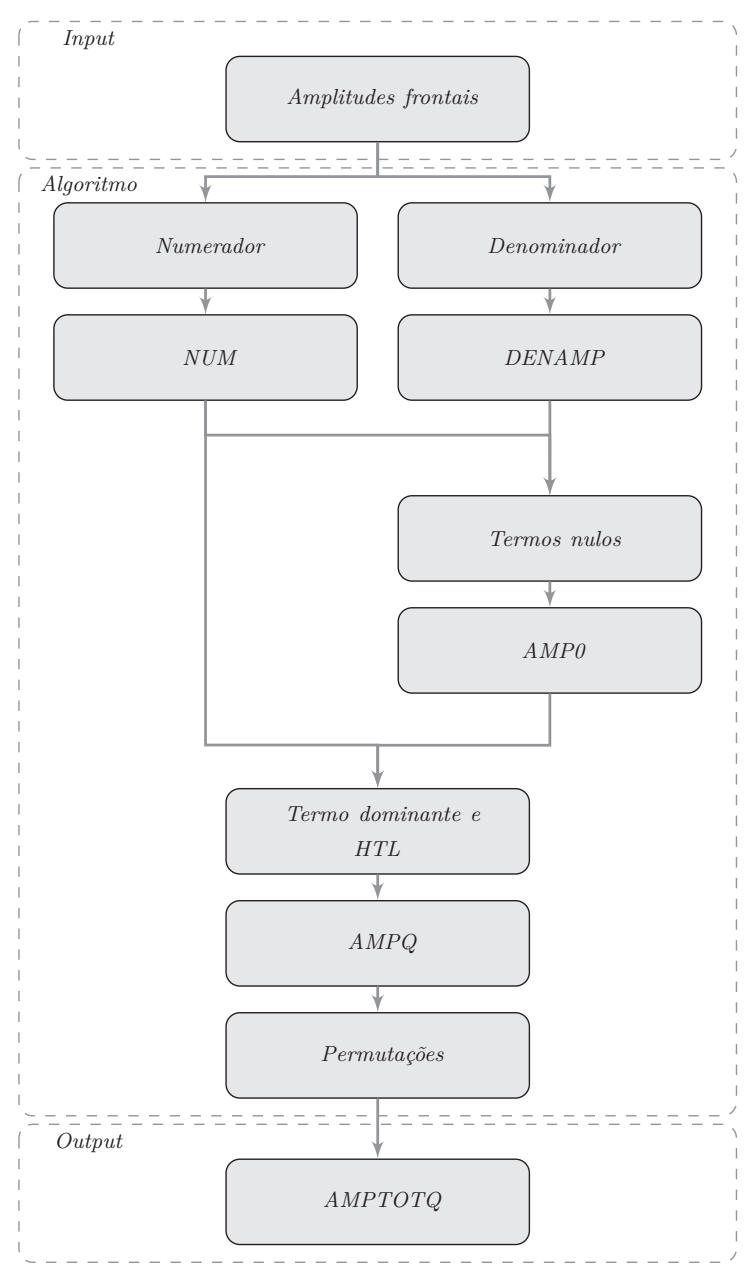

Figura 19 - Fluxograma do algoritmo utilizado no cálculo do limite de altas temperaturas das correções do propagador de um fóton, em um plasma no âmbito da QED.

conservação dos quadrimomentos. Os resultados dos cálculos estão presentes na figura 21, onde podemos observar que a quantidade de termos aumentou expressivamente em relação às amplitudes com apenas duas pernas externas.

Esse aumento expressivo se torna ainda mais evidente no caso de 4 pernas externas, como pode ser observado no resultado final dos cálculos expressos na figura 22, onde vemos que apenas uma única amplitude frontal possui quase 20000 termos. Por este mesmo motivo, os outros outputs na figura 22 foram omitidos, e tivemos que utilizar outras duas funções $A M P Q P E R M 1$ e AMPQPERM2 para auxiliar nas permutações, pois caso contrário deveríamos escrever 24 termos.

\section{E.2 Equação de transporte de Boltzmann}

Já para o formalismo clássico, não existe a necessidade de expansões, desta forma os cálculos são mais simples, bastando apenas efetuar o desmembramento da expressão (6.9), após feitas as iterações, nos seus vários sub-termos. 
A fim de comparar os resultados desta seção com os da seção anterior, utilizaremos nos passos a seguir o tensor eletromagnético no espaço dos momentos,

$$
F\left[p_{\_}, m_{\_}, n_{\_}, \mu_{\_}\right]=F V[p, m] G[n, \mu]-F V[p, n] G[m, \mu] .
$$

Além destes, teremos os tensores $T^{n}$, que dependem inteiramente dos momentos internos. E como sua forma varia de acordo com a ordem da iteração, e não existe uma simples sistemática para expandirmos estes termos ordem a ordem, não será possível exibir uma expressão de forma genérica para os mesmos, sendo que os representaremos por

$$
T N\left[k_{\ldots}, m 1_{-}, m 2_{\Perp}, m 3_{\ldots}, \ldots\right] .
$$

Essas duas funções, (E.8) e (E.9), apresentadas até agora, serão suficientes para o cálculo das amplitudes no presente formalismo, sendo que sob os tensores eletromagnéticos ainda atuarão alguns operadores, que quando no espaço de configurações, possuem derivadas espaciais, assim gerando alguns termos dependentes dos momentos externos.

$$
A M P C\left[p 1_{\ldots}, p 2_{\ldots}, p 3 \ldots, \ldots, \mu 1 \_, \mu 2 \_, \mu 3 \ldots, \ldots\right]
$$

Da mesma forma como não foi possível explicitar uma forma genérica para $T^{n}$, não será possível definirmos uma expressão geral para as amplitudes (E.10) sem especificarmos a ordem de iteração.

Ademais, a expressão (E.10) configura apenas uma das possibilidades para as amplitudes, já que existe uma arbitrariedade ao numerarmos os momentos em (6.22), portanto, devemos simetrizar este resultado através da soma de todas as permutações cíclicas possíveis,

$$
\begin{aligned}
& A M P C S Y M\left[p 1_{\ldots}, p 2_{\ldots}, p 3_{\ldots}, \ldots, \mu 1 \_, \mu 2_{\ldots}, \mu 3 \ldots, \ldots\right]= \\
& \begin{aligned}
\{A M P C[p 1, p 2, p 3, \ldots, \mu 1, \mu 2, \mu 3, \ldots]+A M P C[p 2, p 3, \ldots, p 1, \mu 2, \mu 3, \ldots, \mu 1]+ \\
+A M P C[p 3, p 4, \ldots, p 1, p 2, \mu 3, \mu 4, \ldots, \mu 1, \mu 2]+\ldots\} .
\end{aligned}
\end{aligned}
$$

Mas como essa expressão foi obtida a partir de uma teoria clássica, devemos ainda corrigi-la por um fator que leva em consideração os graus de liberdade do spin, cujo valor é igual a 2 para $3+1$ dimensões espaço-temporais, segundo (5.31).

Novamente, podemos resumir os procedimentos descritos nesta seção através de um fluxograma, figura 20, cujo input é proveniente das iterações de (6.22) até a ordem desejada. Da mesma forma como ocorre no fluxograma da figura 19, teremos como output a amplitude térmica total. 


$$
\begin{aligned}
& \text { AMPTOTC }\left[p 1_{\_}, p 2 \_, p 3 \ldots, \ldots, \mu 1 \_, \mu 2 \_, \mu 3 \ldots, \ldots\right]= \\
& \text { fator do } \operatorname{spin} \times A M P C S Y M\left[p 1 \_, p 2 \_, p 3 \ldots, \ldots, \mu 1 \_, \mu 2 \_, \mu 3 \ldots, \ldots\right]
\end{aligned}
$$

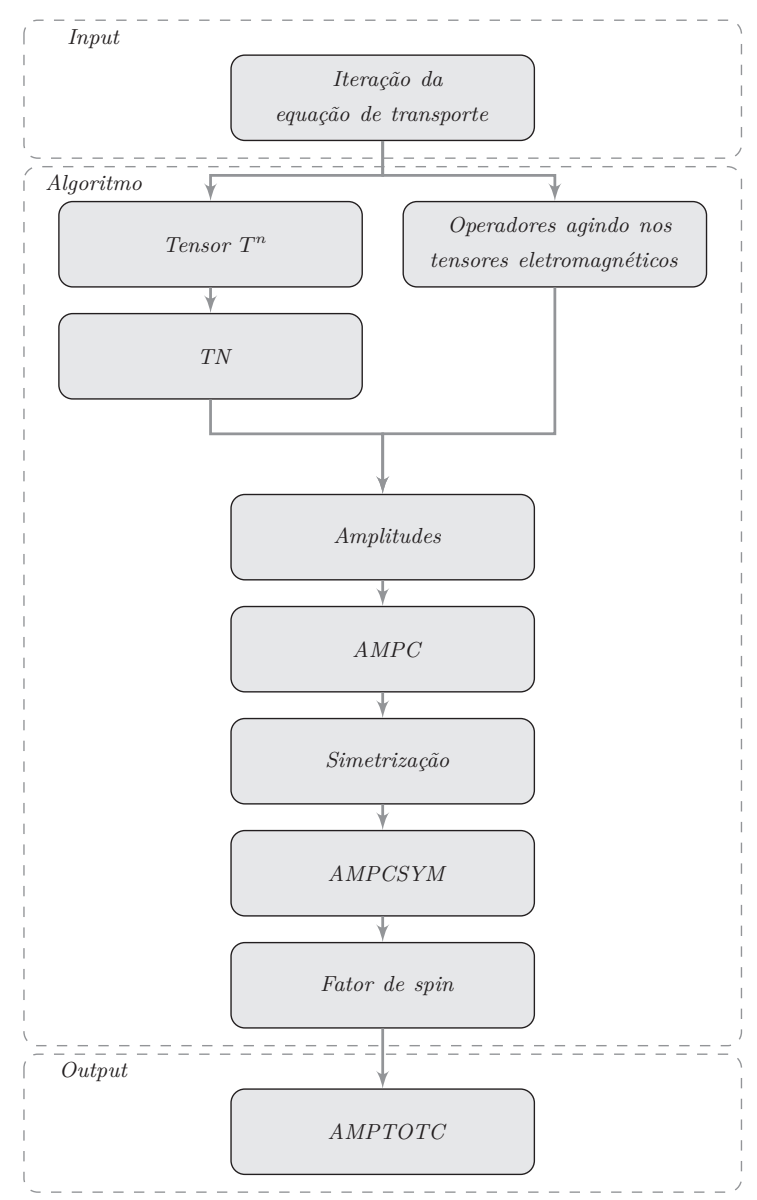

Figura 20 - Fluxograma do algoritmo utilizado nos cálculos das amplitudes térmicas via iterações da equação de transporte de Boltzmann, para um plasma eletrônico.

Assim como fizemos na seção anterior, ao aplicarmos este algoritmo para a terceira e quarta ordens de iteração da equação de Boltzmann, obtemos os resultados expostos nas figuras 23 e 24.

\section{E.3 Comparação entre os resultados}

Por fim, podemos comparar os dois resultados através de

$$
\begin{array}{r}
\frac{1}{n} A M P T O T C\left[p 1 \_, p 2 \_, p 3 \_, \ldots, \mu 1 \_, \mu 2 \_, \mu 3 \_, . .\right]-A M P T O T Q\left[p 1 \_, p 2 \_, p 3 \_, \ldots\right. \\
\left.\mu 1 \_, \mu 2 \_, \mu 3 \_, \ldots\right] ; \quad(\mathrm{E} .13)
\end{array}
$$


em que o fator $\frac{1}{n}$ é proveniente de (6.42). Adicionalmente, por uma limitação do Mathematica, deveremos efetuar o comando abaixo, logo em seguida ao cálculo acima.

$$
\text { ExpandScalarProduct }[\%] \cdot / p_{n} \rightarrow-p_{n-1}-p_{n-2}-\ldots-p_{1}
$$

Vale salientar, que na comparação (E.13), assim como em outros passos, foi necessário incluir a função ExpandScalar Product no algoritmo, devido ao FeynCalc não utilizar automaticamente a propriedade distributiva dos quadritensores, FourVector $[k+$ $p, \mu]=$ FourVector $[k, \mu]+$ FourVector $[p, \mu]$.

Este procedimento, como é exposto nas figuras 25 e 26, foi aplicado para $n=3$ e 4 , onde obtivemos (E.14) igual a 0 em ambos os casos, o que confirma uma concordância entre o formalismo clássico e o quântico até a quarta ordem de aproximação. 


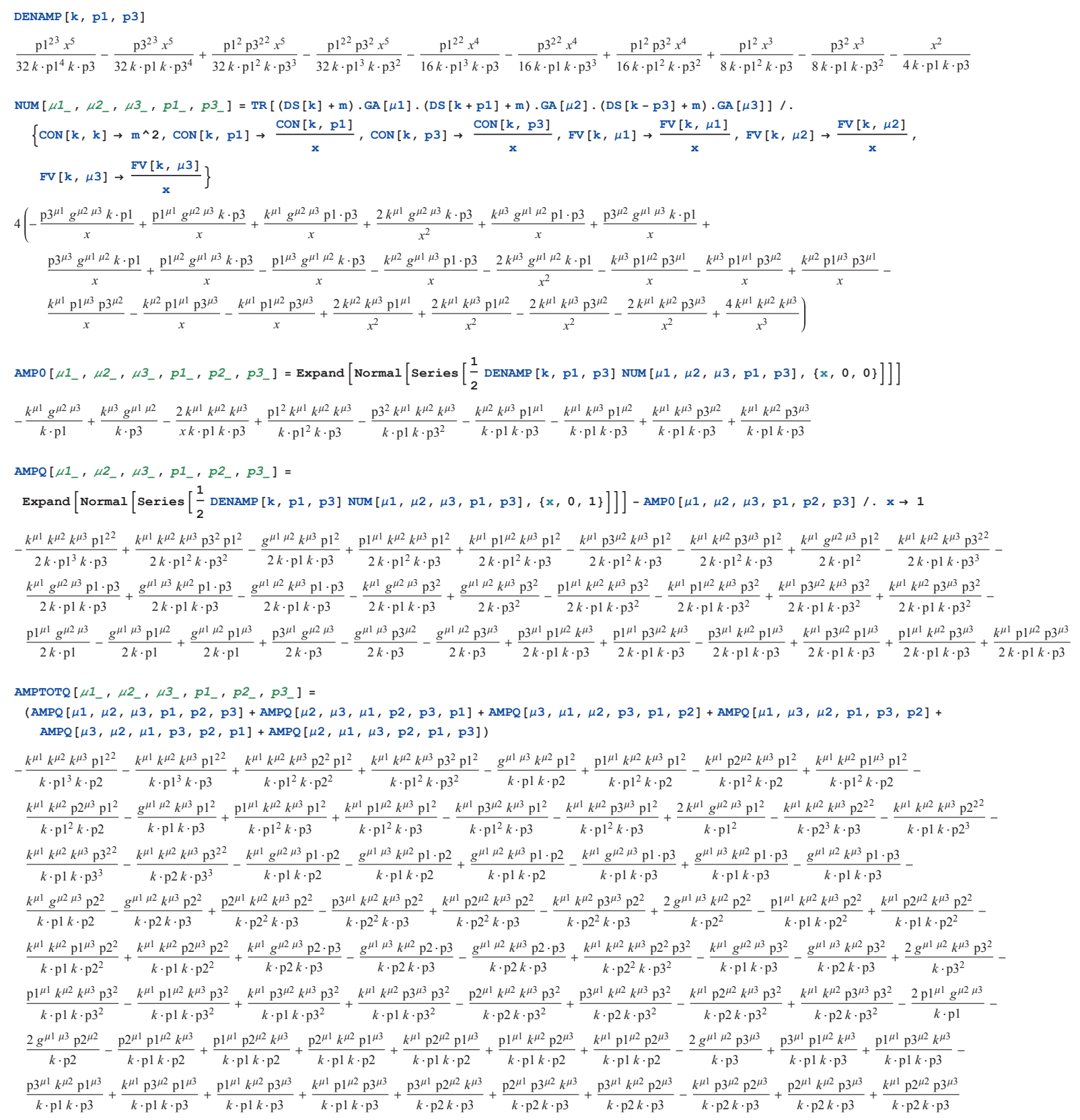

Figura 21 - Cálculos efetuados via o aplicativo Mathematica para uma amplitude térmica com 3 pernas externas seguindo o algoritmo da figura 19, onde os comandos, texto em azul, estão seguidos por seus respectivos outputs. 


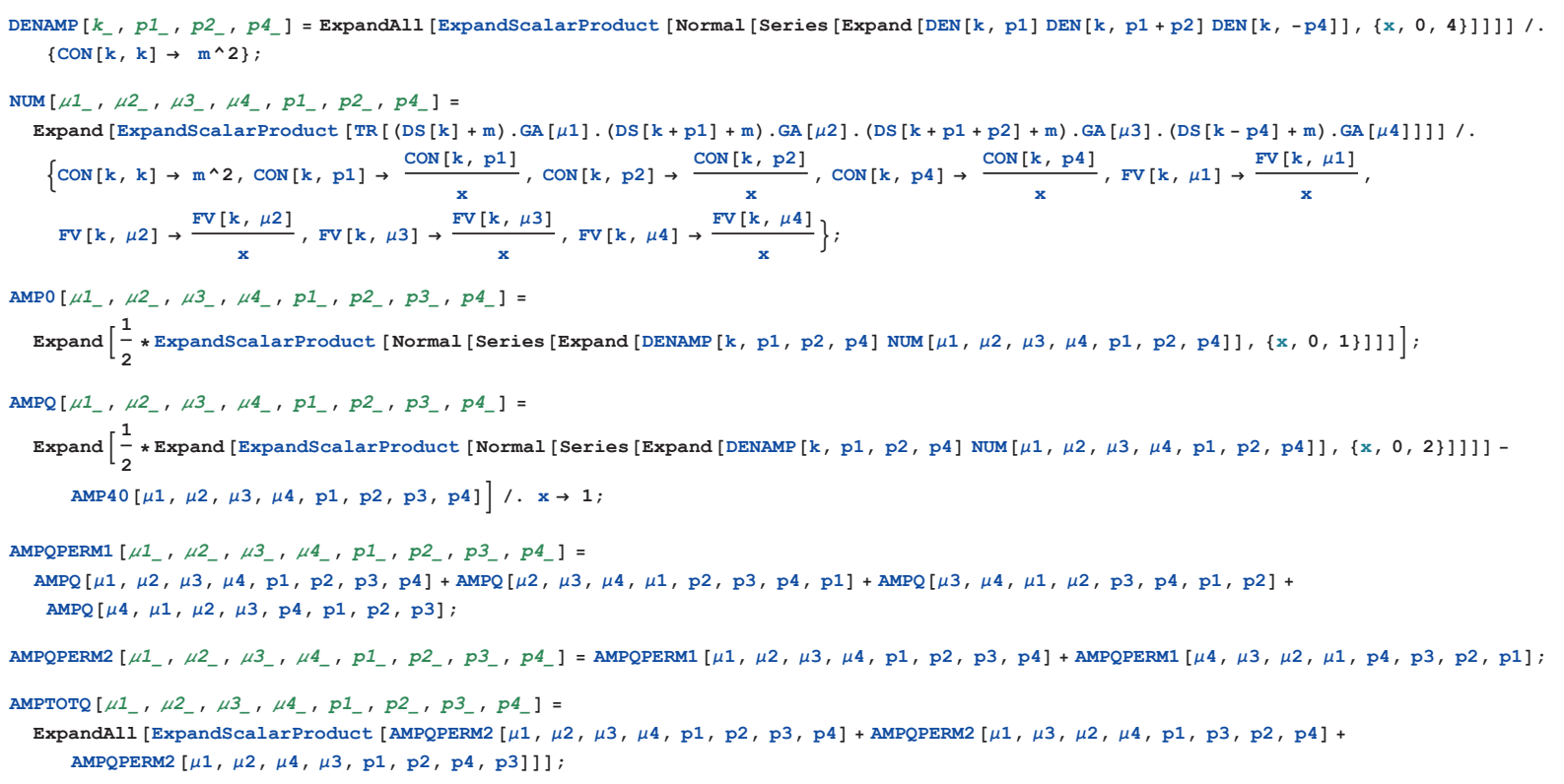

Figura 22 - Cálculos efetuados via o aplicativo Mathematica para uma amplitude térmica com 4 pernas externas seguindo o algoritmo da figura 19, onde os comandos, texto em azul, estão seguidos por seus respectivos outputs. 
$\operatorname{TN}\left[k_{-}, \mu 1_{-}, \nu 1_{-}, \mu 2_{-}, \nu 2_{-}, \mu 3_{-}, \nu 3_{-}, \lambda 1_{-}, \lambda 2_{-}, \lambda 3_{-}\right]=$

$2 \operatorname{FV}[k, \mu 1] \operatorname{FV}[k, \mu 2] \operatorname{FV}[k, \mu 3] \operatorname{FV}[k, \lambda 2] \operatorname{FV}[k, \lambda 3] \mathrm{G}[v 1, \nu 2] \mathrm{G}[v 3, \lambda 1]+$

$\operatorname{FV}[k, \mu 1] \operatorname{FV}[k, \mu 2] \operatorname{FV}[k, \mu 3] \operatorname{FV}[k, \lambda 1] \operatorname{FV}[k, \lambda 2] \mathrm{G}[v 1, \nu 2] \mathrm{G}\left[v_{3}, \lambda 3\right]-$

$\operatorname{FV}[k, \mu 1] \operatorname{FV}[k, \mu 3] \operatorname{FV}[k, \lambda 1] \operatorname{FV}[k, \lambda 2] \operatorname{FV}[k, \lambda 3] \mathrm{G}[v 1, \gamma 2] \mathrm{G}[v 3, \mu 2]-\operatorname{FV}[k, \mu 2] \operatorname{FV}[k, \mu 3] \mathrm{FV}[k, \lambda 1] \mathrm{FV}[k, \lambda 2] \mathrm{FV}[k, \lambda 3] \mathrm{G}[v 1, \gamma 2] \mathrm{G}[\mu 1, \nu 3]$ $-k^{\lambda 1} k^{\lambda 2} k^{\lambda 3} k^{\mu 1} k^{\mu 3} g^{\mu 2 v 3} g^{\nu 1 \nu 2}-k^{\lambda 1} k^{\lambda 2} k^{\lambda 3} k^{\mu 2} k^{\mu 3} g^{\mu 1 \nu 3} g^{\nu 1 \nu 2}+k^{\lambda 1} k^{\lambda 2} k^{\mu 1} k^{\mu 2} k^{\mu 3} g^{\lambda 3 \nu 3} g^{\nu 1 v 2}+2 k^{\lambda 2} k^{\lambda 3} k^{\mu 1} k^{\mu 2} k^{\mu 3} g^{\lambda 1 v 3} g^{\nu 1 \nu 2}$

$\operatorname{AMPC}\left[\mu 1_{-}, \mu 2_{-}, \mu 3_{-}, p 1_{-}, p 2_{-}, p 3_{-}\right]=$

Expand [

Contract [FV [p3, 1$] \mathrm{F}[\mathrm{p} 3, \delta 1, \gamma 1, \mu 3] \mathrm{F}[\mathrm{p} 2, \delta 2, \nu 2, \mu 2] \mathrm{F}[\mathrm{p} 1, \delta 3, \nu 3, \mu 1] \mathrm{FV}[\mathrm{p} 1, \lambda 2] \mathrm{FV}[\mathrm{p} 1, \lambda 3] \mathrm{TN}[\mathrm{k}, \delta 1, \nu 1, \delta 2, \nu 2, \delta 3, \nu 3, \lambda 1, \lambda 2, \lambda 3]] /$ $\left.\operatorname{CON}[k, \mathrm{p} 1]^{\wedge} 3 / \operatorname{CON}\left[k, \mathrm{p}^{3}\right]^{\wedge} 3\right]$

$-\frac{k^{\mu 1} k \cdot \mathrm{p} 2 \mathrm{p}^{2} g^{\mu 2 \mu 3}}{k \cdot \mathrm{p} 1^{2} k \cdot \mathrm{p} 3}+\frac{k^{\mu 1} \mathrm{p} 1 \cdot \mathrm{p} 2 g^{\mu 2 \mu 3}}{k \cdot \mathrm{p} 1 k \cdot \mathrm{p} 3}-\frac{k^{\mu 1} k \cdot \mathrm{p} 2 \mathrm{p} 1 \cdot \mathrm{p} 3 g^{\mu 2 \mu 3}}{k \cdot \mathrm{p} 1 k \cdot \mathrm{p} 3^{2}}-\frac{\mathrm{p}^{\mu 1} g^{\mu 2 \mu 3}}{k \cdot \mathrm{p} 3}+\frac{\mathrm{p}^{\mu 1} k \cdot \mathrm{p} 2 g^{\mu 2 \mu 3}}{k \cdot \mathrm{p} 1 k \cdot \mathrm{p} 3}+\frac{\mathrm{p} 3^{\mu 1} k \cdot \mathrm{p} 2 g^{\mu 2 \mu 3}}{k \cdot \mathrm{p} 3^{2}}+\frac{k^{\mu 1} k^{\mu 2} \mathrm{p}^{\mu 3} \mathrm{p}^{2}}{k \cdot \mathrm{p} 1^{2} k \cdot \mathrm{p} 3}+$

$\frac{k^{\mu 1} \mathrm{p} 3^{\mu 2} k^{\mu 3} k \cdot \mathrm{p} 2 \mathrm{p}^{2}}{k \cdot \mathrm{p} 1^{2} k \cdot \mathrm{p} 3^{2}}-\frac{k^{\mu 1} \mathrm{p} 3^{\mu 2} k^{\mu 3} \mathrm{p} 1 \cdot \mathrm{p} 2}{k \cdot \mathrm{p} 1 k \cdot \mathrm{p} 3^{2}}+\frac{k^{\mu 1} k^{\mu 2} \mathrm{p}^{\mu 3} \mathrm{p} 1 \cdot \mathrm{p} 3}{k \cdot \mathrm{p} 1 k \cdot \mathrm{p} 3^{2}}+\frac{2 k^{\mu 1} \mathrm{p}^{\mu 2} k^{\mu 3} k \cdot \mathrm{p} 2 \mathrm{p} 1 \cdot \mathrm{p} 3}{k \cdot \mathrm{p} 1 k \cdot \mathrm{p} 3^{3}}-\frac{k^{\mu 1} k^{\mu 2} k^{\mu 3} \mathrm{pl}^{2} \mathrm{p} 2 \cdot \mathrm{p} 3}{k \cdot \mathrm{p} 1^{2} k \cdot \mathrm{p} 3^{2}}-\frac{2 k^{\mu 1} k^{\mu 2} k^{\mu 3} \mathrm{p} 1 \cdot \mathrm{p} 3 \mathrm{p} 2 \cdot \mathrm{p} 3}{k \cdot \mathrm{p} 1 k \cdot \mathrm{p} 3^{3}}-$

$\frac{g^{\mu 1 \mu 3} k^{\mu 2} \mathrm{p} 2 \cdot \mathrm{p} 3}{k \cdot \mathrm{p} 3^{2}}-\frac{g^{\mu 1 \mu 2} k^{\mu 3} \mathrm{p} 2 \cdot \mathrm{p} 3}{k \cdot \mathrm{p} 3^{2}}+\frac{\mathrm{p} \mu^{\mu 1} k^{\mu 2} k^{\mu 3} \mathrm{p} 2 \cdot \mathrm{p} 3}{k \cdot \mathrm{p} 1 k \cdot \mathrm{p} 3^{2}}+\frac{k^{\mu 1} \mathrm{p}^{\mu 2} k^{\mu 3} \mathrm{p} 2 \cdot \mathrm{p} 3}{k \cdot \mathrm{p} 1 k \cdot \mathrm{p} 3^{2}}+\frac{k^{\mu 1} k^{\mu 2} \mathrm{p}^{\mu 3} \mathrm{p} 2 \cdot \mathrm{p} 3}{k \cdot \mathrm{p} 1 k \cdot \mathrm{p} 3^{2}}+\frac{2 \mathrm{p}^{\mu 1} k^{\mu 2} k^{\mu 3} \mathrm{p} 2 \cdot \mathrm{p} 3}{k \cdot \mathrm{p} 3^{3}}+\frac{g^{\mu 1 \mu 2} \mathrm{p}^{\mu 3}}{k \cdot \mathrm{p} 3}-$

$\frac{\mathrm{p}{ }^{\mu 1} k^{\mu 2} \mathrm{p}^{\mu 3}}{k \cdot \mathrm{p} 1 k \cdot \mathrm{p} 3}-\frac{k^{\mu 1} \mathrm{p}^{\mu 2} \mathrm{p}^{\mu 3}}{k \cdot \mathrm{p} 1 k \cdot \mathrm{p} 3}+\frac{\mathrm{p} 2^{\mu 1} \mathrm{p}^{\mu 2} k^{\mu 3}}{k \cdot \mathrm{p} 3^{2}}-\frac{\mathrm{p}^{\mu 1} k^{\mu 2} \mathrm{p}^{\mu 3}}{k \cdot \mathrm{p} 3^{2}}+\frac{g^{\mu 1 \mu 3} \mathrm{p} 3^{\mu 2} k \cdot \mathrm{p} 2}{k \cdot \mathrm{p} 3^{2}}-\frac{\mathrm{p} 1^{\mu 1} \mathrm{p}^{\mu 2} k^{\mu 3} k \cdot \mathrm{p} 2}{k \cdot \mathrm{p} 1 k \cdot \mathrm{p} 3^{2}}-\frac{k^{\mu 1} \mathrm{p}^{\mu 2} \mathrm{p}^{\mu 3} k \cdot \mathrm{p} 2}{k \cdot \mathrm{p} 1 k \cdot \mathrm{p} 3^{2}}-\frac{2 \mathrm{p} 3^{\mu 1} \mathrm{p}^{\mu 2} k^{\mu 3} k \cdot \mathrm{p} 2}{k \cdot \mathrm{p} 3^{3}}$

$\operatorname{AMPSYM}\left[\mu 1, \mu 2, \mu 3_{-}, p 1, p 2_{-}, p 3_{-}\right]=$

$(\operatorname{AMPC}[\mu 1, \mu 2, \mu 3, \mathrm{p} 1, \mathrm{p} 2, \mathrm{p} 3]+\operatorname{AMPC}[\mu 2, \mu 3, \mu 1, \mathrm{p} 2, \mathrm{p} 3, \mathrm{p} 1]+\operatorname{AMPC}[\mu 3, \mu 1, \mu 2, \mathrm{p} 3, \mathrm{p} 1, \mathrm{p} 2]+\operatorname{AMPC}[\mu 1, \mu 3, \mu 2, \mathrm{p} 1, \mathrm{p} 3, \mathrm{p} 2]+$

$\operatorname{AMPC}[\mu 3, \mu 2, \mu 1, \mathrm{p} 3, \mathrm{p} 2, \mathrm{p} 1]+\operatorname{AMPC}[\mu 2, \mu 1, \mu 3, \mathrm{p} 2, \mathrm{p} 1, \mathrm{p} 3])$

$\frac{\mathrm{p} 1^{\mu 1} k \cdot \mathrm{p} 3 g^{\mu 2 \mu 3}}{k \cdot \mathrm{p} 1 k \cdot \mathrm{p} 2}+\frac{\mathrm{p} 2^{\mu 1} k \cdot \mathrm{p} 3 g^{\mu 2 \mu 3}}{k \cdot \mathrm{p} 2^{2}}-\frac{k^{\mu 1} k \cdot \mathrm{p} 3 \mathrm{p}^{2} g^{\mu 2 \mu 3}}{k \cdot \mathrm{p} 1^{2} k \cdot \mathrm{p} 2}-\frac{k^{\mu 1} k \cdot \mathrm{p} 2 \mathrm{p}^{2} g^{\mu 2 \mu 3}}{k \cdot \mathrm{p} 1^{2} k \cdot \mathrm{p} 3}-\frac{k^{\mu 1} k \cdot \mathrm{p} 3 \mathrm{p} 1 \cdot \mathrm{p} 2 g^{\mu 2 \mu 3}}{k \cdot \mathrm{p} 1 k \cdot \mathrm{p} 2^{2}}+\frac{k^{\mu 1} \mathrm{p} 1 \cdot \mathrm{p} 2 g^{\mu 2 \mu 3}}{k \cdot \mathrm{p} 1 k \cdot \mathrm{p} 3}-\frac{k^{\mu 1} \mathrm{p} 1 \cdot \mathrm{p} 2 g^{\mu 2 \mu 3}}{k \cdot \mathrm{p} 1^{2}}-\frac{k^{\mu 1} \mathrm{p} 1 \cdot \mathrm{p} 2 g^{\mu 2 \mu 3}}{k \cdot \mathrm{p} 2^{2}}+\frac{k^{\mu 1} \mathrm{p} 1 \cdot \mathrm{p} 3 g^{\mu 2 \mu 3}}{k \cdot \mathrm{p} 1 k \cdot \mathrm{p} 2}-$

$\frac{k^{\mu 1} \mathrm{p} 1 \cdot \mathrm{p} 3 g^{\mu 2 \mu 3}}{k \cdot \mathrm{p} 1^{2}}-\frac{k^{\mu 1} \mathrm{p} 1 \cdot \mathrm{p} 3 g^{\mu 2 \mu 3}}{k \cdot \mathrm{p} 3^{2}}-\frac{k^{\mu 1} k \cdot \mathrm{p} 2 \mathrm{p} 1 \cdot \mathrm{p} 3 g^{\mu 2 \mu 3}}{k \cdot \mathrm{p} 1 k \cdot \mathrm{p} 3^{2}}+\frac{\mathrm{p}^{\mu 1} g^{\mu 2 \mu 3}}{k \cdot \mathrm{p} 1}+\frac{\mathrm{p} 3^{\mu 1} g^{\mu 2 \mu 3}}{k \cdot \mathrm{p} 1}-\frac{\mathrm{p} 3^{\mu 1} g^{\mu 2 \mu 3}}{k \cdot \mathrm{p} 2}-\frac{\mathrm{p} 2^{\mu 1} g^{\mu 2 \mu 3}}{k \cdot \mathrm{p} 3}+\frac{\mathrm{p} 1^{\mu 1} k \cdot \mathrm{p} 2 g^{\mu 2 \mu 3}}{k \cdot \mathrm{p} 1 k \cdot \mathrm{p} 3}+\frac{\mathrm{p} 2^{\mu 1} k \cdot \mathrm{p} 1 g^{\mu 2 \mu 3}}{k \cdot \mathrm{p} 2^{2}}+$

$\frac{\mathrm{p} 3^{\mu 1} k \cdot \mathrm{p} 1 g^{\mu 2 \mu 3}}{k \cdot \mathrm{p} 3^{2}}+\frac{\mathrm{p} 3^{\mu 1} k \cdot \mathrm{p} 2 g^{\mu 2 \mu 3}}{k \cdot \mathrm{p} 3^{2}}+\frac{g^{\mu 1 \mu 3} \mathrm{p}^{\mu 2} k \cdot \mathrm{p} 2}{k \cdot \mathrm{p} 1^{2}}+\frac{g^{\mu 1 \mu 2} \mathrm{p}^{\mu 3} k \cdot \mathrm{p} 2}{k \cdot \mathrm{p} 1^{2}}-\frac{2 k^{\mu 1} \mathrm{p}^{\mu 2} \mathrm{p1}^{\mu 3} k \cdot \mathrm{p} 2}{k \cdot \mathrm{p} 1^{3}}+\frac{g^{\mu 1 \mu 3} \mathrm{p}^{\mu 2} k \cdot \mathrm{p} 3}{k \cdot \mathrm{p} 1 k \cdot \mathrm{p} 2}-\frac{\mathrm{p}^{\mu 1} k^{\mu 2} \mathrm{p1}^{\mu 3} k \cdot \mathrm{p} 3}{k \cdot \mathrm{p} 1^{2} k \cdot \mathrm{p} 2}-\frac{k^{\mu 1} \mathrm{p}^{\mu 2} \mathrm{p1}^{\mu 3} k \cdot \mathrm{p} 3}{k \cdot \mathrm{p} 1^{2} k \cdot \mathrm{p} 2}+$

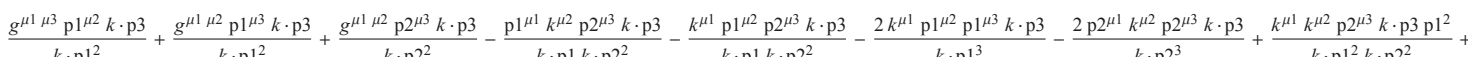

$k \cdot \mathrm{p} 1 k \cdot \mathrm{p} 2^{2} \quad k \cdot \mathrm{p} 1 k \cdot \mathrm{p} 2^{2}$

$\frac{k^{\mu 1} \mathrm{p} 3^{\mu 2} k^{\mu 3} \mathrm{p}^{2}}{k \cdot \mathrm{p} 1^{2} k \cdot \mathrm{p} 2}+\frac{k^{\mu 1} k^{\mu 2} \mathrm{p}^{\mu 3} \mathrm{p}^{2}}{k \cdot \mathrm{p} 1^{2} k \cdot \mathrm{p} 3}+\frac{k^{\mu 1} \mathrm{p}^{\mu 2} k^{\mu 3} k \cdot \mathrm{p} 2 \mathrm{p}^{2}}{k \cdot \mathrm{pl}^{2} k \cdot \mathrm{p} 3^{2}}-\frac{g^{\mu 1} \mu^{3} k^{\mu 2} k \cdot \mathrm{p} 3 \mathrm{p} 1 \cdot \mathrm{p} 2}{k \cdot \mathrm{p} 1^{2} k \cdot \mathrm{p} 2}+\frac{2 k^{\mu 1} k^{\mu 2} \mathrm{p}^{\mu 3} k \cdot \mathrm{p} 3 \mathrm{p} 1 \cdot \mathrm{p} 2}{k \cdot \mathrm{p} 1^{3} k \cdot \mathrm{p} 2}+\frac{2 k^{\mu 1} k^{\mu 2} \mathrm{p}^{\mu 3} k \cdot \mathrm{p} 3 \mathrm{p} 1 \cdot \mathrm{p} 2}{k \cdot \mathrm{p} 1 k \cdot \mathrm{p} 2^{3}}+\frac{\mathrm{p} 3^{\mu 1} k^{\mu 2} k^{\mu 3} \mathrm{p} 1 \cdot \mathrm{p} 2}{k \cdot \mathrm{p} 1^{2} k \cdot \mathrm{p} 2}+$

$\frac{g^{\mu 1 \mu 3} k^{\mu 2} \mathrm{p} 1 \cdot \mathrm{p} 2}{k}+\frac{\mathrm{p} 3^{\mu 1} k^{\mu 2} k^{\mu 3} \mathrm{p} 1 \cdot \mathrm{p} 2}{k \cdot 1^{2} k \cdot \mathrm{p} 3}+\frac{k^{\mu 1} \mathrm{p}^{\mu 2} k^{\mu 3} \mathrm{p} 1 \cdot \mathrm{p} 2}{k \cdot \mathrm{p}^{2} k \cdot \mathrm{p} 3}+\frac{k^{\mu 1} k^{\mu 2} \mathrm{p}^{\mu 3} \mathrm{p} 1 \cdot \mathrm{p} 2}{k \cdot \mathrm{p} 1^{2} k \cdot \mathrm{p} 3}+\frac{\mathrm{p}^{\mu 1} k^{\mu 2} k^{\mu 3} \mathrm{p} 1 \cdot \mathrm{p} 2}{k \cdot \mathrm{p} 2^{2} k \cdot \mathrm{p} 3}+\frac{k^{\mu 1} \mathrm{p}^{\mu 2} k^{\mu 3} \mathrm{p} 1 \cdot \mathrm{p} 2}{k \cdot \mathrm{p} 2^{2} k \cdot \mathrm{p} 3}+\frac{k^{\mu 1} k^{\mu 2} \mathrm{p}^{\mu 3} \mathrm{p} 1 \cdot \mathrm{p} 2}{k \cdot \mathrm{p}^{2} k \cdot \mathrm{p} 3}-\frac{g^{\mu 1 \mu 3} k^{\mu 2} \mathrm{p} 1 \cdot \mathrm{p} 2}{k \cdot \mathrm{p} 1^{2}}-$

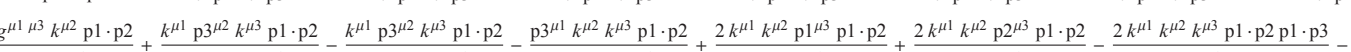

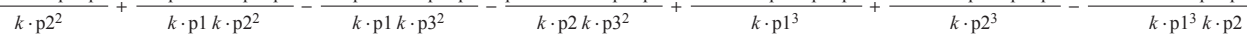
$\frac{2 k^{\mu 1} k^{\mu 2} k^{\mu 3} \mathrm{p} 1 \cdot \mathrm{p} 2 \mathrm{p} 1 \cdot \mathrm{p} 3}{k \cdot \mathrm{p} 1^{3} k \cdot \mathrm{p} 3}+\frac{\mathrm{p}^{\mu 1} k^{\mu 2} k^{\mu 3} \mathrm{p} 1 \cdot \mathrm{p} 3}{k \cdot \mathrm{p} 1^{2} k \cdot \mathrm{p} 2}+\frac{k^{\mu 1} \mathrm{p} 2^{\mu 2} k^{\mu 3} \mathrm{p} 1 \cdot \mathrm{p} 3}{k \cdot \mathrm{p} 1^{2} k \cdot \mathrm{p} 2}+\frac{k^{\mu 1} k^{\mu 2} \mathrm{p}^{\mu 3} \mathrm{p} 1 \cdot \mathrm{p} 3}{k \cdot \mathrm{p} 1^{2} k \cdot \mathrm{p} 2}-\frac{g^{\mu 1 \mu 2} k^{\mu 3} k \cdot \mathrm{p} 2 \mathrm{p} 1 \cdot \mathrm{p} 3}{k \cdot \mathrm{p} 1^{2} k \cdot \mathrm{p} 3}+\frac{2 k^{\mu 1} \mathrm{p}^{\mu 2} k^{\mu 3} k \cdot \mathrm{p} 2 \mathrm{p} 1 \cdot \mathrm{p} 3}{k \cdot \mathrm{p} 1^{3} k \cdot \mathrm{p} 3}+\frac{g^{\mu 1 \mu 2} k^{\mu 3} \mathrm{p} 1 \cdot \mathrm{p} 3}{k \cdot \mathrm{p} 2 k \cdot \mathrm{p} 3}+$

$\frac{\mathrm{p} 2^{\mu 1} k^{\mu 2} k^{\mu 3} \mathrm{p} 1 \cdot \mathrm{p} 3}{k \cdot 1^{2} k \cdot \mathrm{p} 3}-\frac{2^{\mu 1} k^{\mu 2} k^{\mu 3} \mathrm{p} 1 \cdot \mathrm{p} 3}{k \cdot 2^{2} k \cdot \mathrm{p} 3}-\frac{g^{\mu 1 \mu 2} k^{\mu 3} \mathrm{p} 1 \cdot \mathrm{p} 3}{k \cdot \mathrm{p} 1^{2}}-\frac{k^{\mu 1} k^{\mu 2} \mathrm{p} 2^{\mu 3} \mathrm{p} 1 \cdot \mathrm{p} 3}{k \cdot g^{\mu 1 \mu 2} k^{\mu 3} \mathrm{p} 1 \cdot \mathrm{p} 3}+\frac{k^{\mu 1} k^{\mu 2} \mathrm{p} 2^{\mu 3} \mathrm{p} 1 \cdot \mathrm{p} 3}{k \cdot \mathrm{p} 3^{2}}+\frac{\mathrm{p} 2^{\mu 1} k^{\mu 2} k^{\mu 3} \mathrm{p} 1 \cdot \mathrm{p} 3}{k \cdot \mathrm{p} 2 k \cdot \mathrm{p} 3^{2}}+\frac{k^{\mu 1} \mathrm{p} 2^{\mu 2} k^{\mu 3} \mathrm{p} 1 \cdot \mathrm{p} 3}{k \cdot \mathrm{p} 2 k \cdot \mathrm{p} 3^{2}}+$ $\frac{k^{\mu 1} k^{\mu 2} \mathrm{p} 2^{\mu 3} \mathrm{p} 1 \cdot \mathrm{p} 3}{k}+\frac{2 k^{\mu 1} \mathrm{p} 1^{\mu 2} k^{\mu 3} \mathrm{p} 1 \cdot \mathrm{p} 3}{k \cdot 2 k^{\mu 1} \mathrm{p}^{\mu 2} k^{\mu 3} \mathrm{p} 1 \cdot \mathrm{p} 3}+\frac{2 k^{\mu 1} \mathrm{p} 3^{\mu 2} k^{\mu 3} k \cdot \mathrm{p} 2 \mathrm{p} 1 \cdot \mathrm{p} 3}{k \cdot 3^{3}}-\frac{g^{\mu 1} \mu 3 k^{\mu 2} k \cdot \mathrm{p} 3 \mathrm{p} 2^{2}}{k \cdot k^{\mu 1} k^{\mu 2} \mathrm{p}^{\mu 3} k \cdot \mathrm{p} 3 \mathrm{p}^{2}}-\frac{k^{\mu 1} k^{\mu 2} k^{\mu 3} \mathrm{p} 1 \cdot \mathrm{p} 3 \mathrm{p} 2^{2}}{k \cdot \mathrm{p} 1^{2} k \cdot \mathrm{p} 2}-$

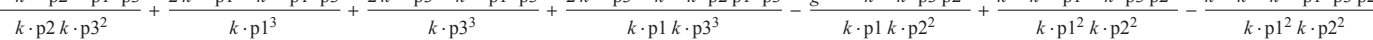
$\frac{k^{\mu 1} k^{\mu 2} k^{\mu 3} \mathrm{p} 1 \cdot \mathrm{p} 3 \mathrm{p}^{2}}{k \cdot \mathrm{p} 2^{2} k \cdot \mathrm{p} 3^{2}}+\frac{k^{\mu 1} k^{\mu 2} \mathrm{p}^{\mu 3} \mathrm{p} 2^{2}}{k \cdot \mathrm{p} 2^{2} k \cdot \mathrm{p} 3}-\frac{g^{\mu 1 \mu 3} k^{\mu 2} k \cdot \mathrm{p} 1 \mathrm{p} 2^{2}}{k \cdot \mathrm{p} 2^{2} k \cdot \mathrm{p} 3}+\frac{\mathrm{p}^{\mu 1} k^{\mu 2} k^{\mu 3} \mathrm{p} 2^{2}}{k \cdot \mathrm{p} 1 k \cdot \mathrm{p} 2^{2}}+\frac{\mathrm{p} 3^{\mu 1} k^{\mu 2} k^{\mu 3} k \cdot \mathrm{p} 1 \mathrm{p} 2^{2}}{k \cdot \mathrm{p} 2^{2} k \cdot \mathrm{p} 3^{2}}-\frac{k^{\mu 1} k^{\mu 2} k^{\mu 3} \mathrm{p} 1^{2} \mathrm{p} 2 \cdot \mathrm{p} 3}{k \cdot \mathrm{p} 1^{2} k \cdot \mathrm{p} 2^{2}}-\frac{k^{\mu 1} k^{\mu 2} k^{\mu 3} \mathrm{p} 1^{2} \mathrm{p} 2 \cdot \mathrm{p} 3}{k \cdot \mathrm{p} 1^{2} k \cdot \mathrm{p} 3^{2}}-$

$\frac{2 k^{\mu 1} k^{\mu 2} k^{\mu 3} \mathrm{p} 1 \cdot \mathrm{p} 2 \mathrm{p} 2 \cdot \mathrm{p} 3}{k}-\frac{2 k^{\mu 1} k^{\mu 2} k^{\mu 3} \mathrm{p} 1 \cdot \mathrm{p} 2 \mathrm{p} 2 \cdot \mathrm{p} 3}{k \cdot \mathrm{p} 1 k \cdot \mathrm{p}^{3}}-\frac{2 k^{\mu 1} k^{\mu 2} k^{\mu 3} \mathrm{p} 1 \cdot \mathrm{p} 3 \mathrm{p} 2 \cdot \mathrm{p} 3}{k \cdot \mathrm{p} 1 \cdot \mathrm{p} 3^{3}}-\frac{2 k^{\mu 1} k^{\mu 2} k^{\mu 3} \mathrm{p} 1 \cdot \mathrm{p} 3 \mathrm{p} 2 \cdot \mathrm{p} 3}{k \cdot g^{\mu}{ }^{\mu} \mu^{3} k^{\mu 2} \mathrm{p} 2 \cdot \mathrm{p} 3}-\frac{k^{\mu 1} k^{\mu 2} \mathrm{p}^{\mu 3} \mathrm{p} 2 \cdot \mathrm{p} 3}{k \cdot \mathrm{p} 1 k \cdot \mathrm{p} 2}+\frac{g^{\mu 1 \mu 2} k^{\mu 3} \mathrm{p} 2 \cdot \mathrm{p} 3}{k \cdot 3^{2}}-$

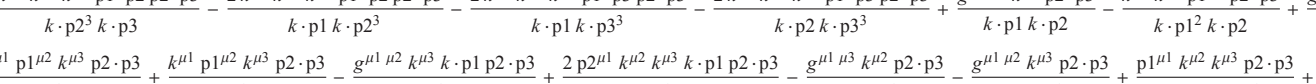

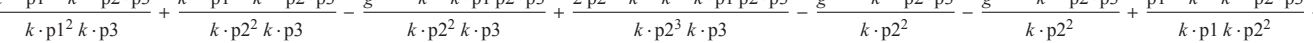

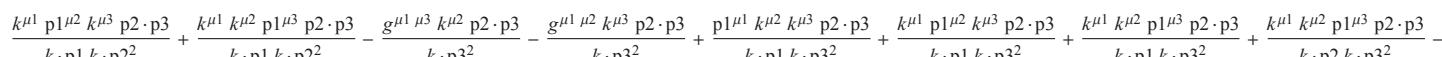
$\frac{g^{\mu 1 \mu 3} k^{\mu 2} k \cdot \mathrm{p} 1 \mathrm{p} 2 \cdot \mathrm{p} 3}{k \cdot \mathrm{p} 2 k \cdot \mathrm{p} 3^{2}}+\frac{2 \mathrm{p} 2^{\mu 1} k^{\mu 2} k^{\mu 3} \mathrm{p} 2 \cdot \mathrm{p} 3}{k \cdot \mathrm{p} 2^{3}}+\frac{2 \mathrm{p} 3^{\mu 1} k^{\mu 2} k^{\mu 3} \mathrm{p} 2 \cdot \mathrm{p} 3}{k \cdot \mathrm{p} 3^{3}}+\frac{2 \mathrm{p} 3^{\mu 1} k^{\mu 2} k^{\mu 3} k \cdot \mathrm{p} 1 \mathrm{p} 2 \cdot \mathrm{p} 3}{k \cdot \mathrm{p} 2 k \cdot \mathrm{p} 3^{3}}-\frac{k^{\mu 1} k^{\mu 2} k^{\mu 3} \mathrm{p} 1 \cdot \mathrm{p} 2 \mathrm{p} 3^{2}}{k \cdot \mathrm{p} 1^{2} k \cdot \mathrm{p} 3^{2}}-\frac{k^{\mu 1} k^{\mu 2} k^{\mu 3} \mathrm{p} 1 \cdot \mathrm{p} 2 \mathrm{p} 3^{2}}{k \cdot \mathrm{p} 2^{2} k \cdot \mathrm{p} 3^{2}}-\frac{g^{\mu 1} \mu 2 k^{\mu 3} k \cdot \mathrm{p} 2 \mathrm{p} 3^{2}}{k \cdot \mathrm{p} 1 k \cdot \mathrm{p} 3^{2}}+$

$\frac{k^{\mu 1} \mathrm{p}^{\mu 2} k^{\mu 3} k \cdot \mathrm{p} 2 \mathrm{p} 3^{2}}{k \cdot \mathrm{p}^{2} k \cdot \mathrm{p} 3^{2}}+\frac{2^{\mu 1} k^{\mu 2} k^{\mu 3} \mathrm{p}^{2}}{k \cdot \mathrm{p} 1 k \cdot \mathrm{p} 3^{2}}+\frac{k^{\mu 1} \mathrm{p}^{\mu 2} k^{\mu 3} \mathrm{p}^{2}}{k \cdot \mathrm{p} 2 k \cdot \mathrm{p} 3^{2}}-\frac{g^{\mu 1 \mu 2} k^{\mu 3} k \cdot \mathrm{p} 1 \mathrm{p} 3^{2}}{k \cdot \mathrm{p} 2 k \cdot \mathrm{p} 3^{2}}+\frac{\mathrm{p}^{2^{\mu 1}} k^{\mu 2} k^{\mu 3} k \cdot \mathrm{p} 1 \mathrm{p} 3^{2}}{k \cdot \mathrm{p} 2^{2} k \cdot \mathrm{p} 3^{2}}-\frac{g^{\mu 1 \mu 3} \mathrm{p}^{\mu 2}}{k \cdot \mathrm{p} 1}-\frac{g^{\mu 1 \mu 2} \mathrm{p}^{\mu 3}}{k \cdot \mathrm{p} 1}+\frac{g^{\mu 1 \mu 3} \mathrm{p}^{\mu 2}}{k \cdot \mathrm{p} 2}+\frac{g^{\mu 1 \mu 3} \mathrm{p}^{\mu 2}}{k \cdot \mathrm{p} 2}-$

$\frac{g^{\mu 1 \mu 2} \mathrm{p}^{\mu 3}}{k \cdot \mathrm{p} 2}-\frac{\mathrm{p} 3^{\mu 1} \mathrm{p}^{\mu 2} k^{\mu 3}}{k \cdot \mathrm{p} 1 k \cdot \mathrm{p} 2}-\frac{\mathrm{p} 1^{\mu 1} \mathrm{p}^{\mu 2} k^{\mu 3}}{k \cdot \mathrm{p} 1 k \cdot \mathrm{p} 2}-\frac{k^{\mu 1} \mathrm{p}^{\mu 2} \mathrm{p}^{\mu 3}}{k \cdot \mathrm{p} 1 k \cdot \mathrm{p} 2}-\frac{\mathrm{p}^{\mu 1} k^{\mu 2} \mathrm{p}^{\mu 3}}{k \cdot \mathrm{p} 1 k \cdot \mathrm{p} 2}-\frac{g^{\mu 1 \mu 3} \mathrm{p}^{\mu 2}}{k \cdot \mathrm{p} 3}+\frac{g^{\mu 1 \mu 2} \mathrm{p}^{\mu 3}}{k \cdot \mathrm{p} 3}+\frac{g^{\mu 1 \mu 2} \mathrm{p}^{\mu 3}}{k \cdot \mathrm{p} 3}+\frac{g^{\mu 1 \mu 2} \mathrm{p}^{\mu 3} k \cdot \mathrm{p} 2}{k \cdot \mathrm{p} 1 k \cdot \mathrm{p} 3}-\frac{\mathrm{p} 3^{\mu 1} \mathrm{p}^{\mu 2} k^{\mu 3} k \cdot \mathrm{p} 2}{k \cdot \mathrm{p} 1^{2} k \cdot \mathrm{p} 3}-$

$\frac{k^{\mu 1} \mathrm{p}^{\mu 2} \mathrm{p}^{\mu 3} k \cdot \mathrm{p} 2}{k \cdot \mathrm{p} 1^{2} k \cdot \mathrm{p} 3}-\frac{\mathrm{p} 2^{\mu 1} \mathrm{p} 3^{\mu 2} k^{\mu 3}}{k \cdot \mathrm{p} 1 k \cdot \mathrm{p} 3}-\frac{\mathrm{p} 1^{\mu 1} k^{\mu 2} \mathrm{p}^{\mu 3}}{k \cdot \mathrm{p} 1 k \cdot \mathrm{p} 3}-\frac{k^{\mu 1} \mathrm{p}^{\mu 2} \mathrm{p}^{\mu 3}}{k \cdot \mathrm{p} 1 k \cdot \mathrm{p} 3}-\frac{\mathrm{p} 2^{\mu 1} k^{\mu 2} \mathrm{p}^{\mu 3}}{k \cdot \mathrm{p} 1 k \cdot \mathrm{p} 3}-\frac{\mathrm{p}^{\mu 1} \mathrm{p}^{\mu 2} k^{\mu 3}}{k \cdot \mathrm{p} 2 k \cdot \mathrm{p} 3}-\frac{\mathrm{p} 2^{\mu 1} k^{\mu 2} \mathrm{p}^{\mu 3}}{k \cdot \mathrm{p} 2 k \cdot \mathrm{p} 3}-\frac{k^{\mu 1} \mathrm{p}^{\mu 2} \mathrm{p}^{\mu 3}}{k \cdot \mathrm{p} 2 k \cdot \mathrm{p} 3}-\frac{k^{\mu 1} \mathrm{p}^{\mu 2} \mathrm{p}^{\mu 3}}{k \cdot \mathrm{p} 2 k \cdot \mathrm{p} 3}+\frac{g^{\mu 1 \mu 3} \mathrm{p}^{\mu 2} k \cdot \mathrm{p} 1}{k \cdot \mathrm{p} 2 k \cdot \mathrm{p} 3}+$

$\frac{g^{\mu 1} \mu 2 \mathrm{p} 3^{\mu 3} k \cdot \mathrm{p} 1}{k \cdot \mathrm{p} 2 k \cdot \mathrm{p} 3}-\frac{\mathrm{p} 2^{\mu 1} \mathrm{p}^{\mu 2} k^{\mu 3} k \cdot \mathrm{p} 1}{k \cdot \mathrm{p} 2^{2} k \cdot \mathrm{p} 3}-\frac{\mathrm{p} 2^{\mu 1} k^{\mu 2} \mathrm{p}^{\mu 3} k \cdot \mathrm{p} 1}{k \cdot \mathrm{p} 2^{2} k \cdot \mathrm{p} 3}-\frac{\mathrm{p} 3^{\mu 1} \mathrm{p}^{\mu 2} k^{\mu 3}}{k \cdot \mathrm{p} 1^{2}}-\frac{\mathrm{p} 2^{\mu 1} k^{\mu 2} \mathrm{p}^{\mu 3}}{k \cdot \mathrm{p} 1^{2}}+\frac{k^{\mu 1} \mathrm{p}^{\mu 2} \mathrm{p}^{\mu 3}}{k \cdot \mathrm{p} 1^{2}}+\frac{k^{\mu 1} \mathrm{p}^{\mu 2} \mathrm{p}^{\mu 3}}{k \cdot \mathrm{p} 1^{2}}-\frac{\mathrm{p}^{\mu 1} \mathrm{p}^{\mu 2} k^{\mu 3}}{k \cdot \mathrm{p} 2^{2}}+\frac{\mathrm{p}^{\mu 1} k^{\mu 2} \mathrm{p}^{\mu 3}}{k \cdot \mathrm{p} 2^{2}}+$

$\frac{\mathrm{p} 3^{\mu 1} k^{\mu 2} \mathrm{p}^{\mu 3}}{k \cdot \mathrm{p} 2^{2}}-\frac{k^{\mu 1} \mathrm{p}^{\mu 2} \mathrm{p}^{\mu 3}}{k \cdot \mathrm{p} 2^{2}}+\frac{g^{\mu 1 \mu 2} \mathrm{p}^{\mu 3} k \cdot \mathrm{p} 1}{k \cdot \mathrm{p} 2^{2}}+\frac{\mathrm{p} 3^{\mu 1} \mathrm{p}^{\mu 2} k^{\mu 3}}{k \cdot \mathrm{p} 3^{2}}+\frac{\mathrm{p} 2^{\mu 1} \mathrm{p}^{\mu 2} k^{\mu 3}}{k \cdot \mathrm{p} 3^{2}}-\frac{k^{\mu 1} \mathrm{p} 3^{\mu 2} \mathrm{p}^{\mu 3}}{k \cdot \mathrm{p} 3^{2}}-\frac{\mathrm{p} 3^{\mu 1} k^{\mu 2} \mathrm{p}^{\mu 3}}{k \cdot \mathrm{p} 3^{2}}+\frac{g^{\mu 1 \mu 3} \mathrm{p} 3^{\mu 2} k \cdot \mathrm{p} 1}{k \cdot \mathrm{p} 3^{2}}+\frac{g^{\mu 1 \mu 3} \mathrm{p} 3^{\mu 2} k \cdot \mathrm{p} 2}{k \cdot \mathrm{p} 3^{2}}-$

$\underline{\mathrm{p} 1^{\mu 1} \mathrm{p}^{\mu 2} k^{\mu 3} k \cdot \mathrm{p} 2}-\frac{k^{\mu 1} \mathrm{p}^{\mu 2} \mathrm{p}^{\mu 3} k \cdot \mathrm{p} 2}{k}-\frac{\mathrm{p} 3^{\mu 1} \mathrm{p} 2^{\mu 2} k^{\mu 3} k \cdot \mathrm{p} 1}{\mathrm{p} 3^{\mu 1} k^{\mu 2} \mathrm{p}^{\mu 3} k \cdot \mathrm{p} 1}-\frac{2 \mathrm{p} 2^{\mu 1} k^{\mu 2} \mathrm{p}^{\mu 3} k \cdot \mathrm{p} 1}{k \cdot \frac{\mathrm{p} 3^{\mu 1}}{\mathrm{p}} 3^{\mu 2} k^{\mu 3} k \cdot \mathrm{p} 1}-\frac{2 \mathrm{p} 3^{\mu 1} \mathrm{p}^{\mu 2} k^{\mu 3} k \cdot \mathrm{p} 2}{k \cdot \mathrm{p} 3^{3}}$

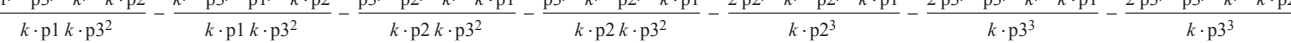

$\operatorname{AMPTOTC}[\mu 1, \mu 2, \mu 3, \mathrm{p} 1, \mathrm{p} 2, \mathrm{p} 3]=2 * \operatorname{AMPSYM}[\mu 1, \mu 2, \mu 3, \mathrm{p} 1, \mathrm{p} 2, \mathrm{p} 3] ;$

Figura 23 - Cálculos efetuados via o aplicativo Mathematica para uma amplitude térmica com 3 pernas externas seguindo o algoritmo da figura 20, onde os comandos, texto em azul, estão seguidos por seus respectivos outputs. 


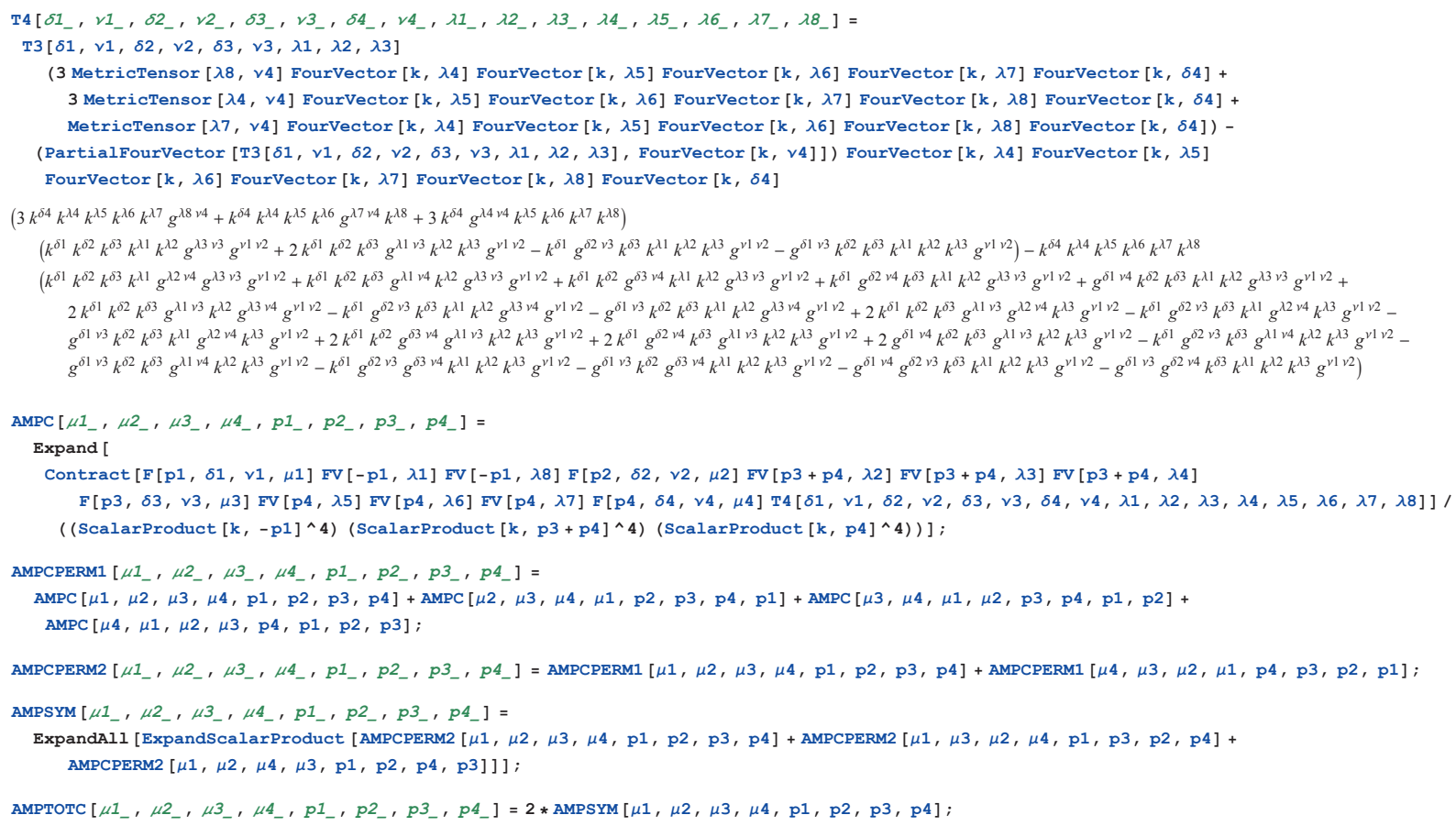

Figura 24 - Cálculos efetuados via o aplicativo Mathematica para uma amplitude térmica com 4 pernas externas seguindo o algoritmo da figura 20, onde os comandos, texto em azul, estão seguidos por seus respectivos outputs.

Expand [AМРтОтQ $\left.[\mu 1, \mu 2, \mu 3, \mathrm{p} 1, \mathrm{p} 2, \mathrm{p} 3]-\frac{1}{3} \operatorname{AMPTOTC}[\mu 1, \mu 2, \mu 3, \mathrm{p} 1, \mathrm{p} 2, \mathrm{p} 3]\right] / . \mathrm{p} 2 \rightarrow-\mathrm{p} 1-\mathrm{p} 3 ;$

Simplify [ExpandScalarProduct [\%] ]

0

Figura 25 - Resultado dos cálculos via o aplicativo Mathematica para uma amplitude térmica com 3 pernas externas, onde os comandos, texto em azul, estão seguidos pelos outputs.

Expand [ExpandScalarProduct $\left[\right.$ АMPTOTQ $\left.\left.[\mu 1, \mu 2, \mu 3, \mu 4, \mathrm{p} 1, \mathrm{p} 2, \mathrm{p} 3, \mathrm{p} 4]-\frac{1}{4} * \operatorname{AMPTOTC}[\mu 1, \mu 2, \mu 3, \mu 4, \mathrm{p} 1, \mathrm{p} 2, \mathrm{p} 3, \mathrm{p} 4]\right]\right] / .\{\mathrm{p} 4 \rightarrow-\mathrm{p} 1-\mathrm{p} 2-\mathrm{p} 3\} ;$ Simplify [\%, TimeConstraint $\rightarrow 10000]$

0

Figura 26 - Resultado dos cálculos via o aplicativo Mathematica para uma amplitude térmica com 4 pernas externas, onde os comandos, texto em azul, estão seguidos pelos outputs. 



\section{Referências}

BARTON, G. On the finite temperature Quantum Electrodynamics of free electrons and photons. Ann. Phys., v. 200, p. 271, 1990. Citado na página 91.

BELLAC, M. L. Thermal Field Theory. Cambridge, England: Cambridge University Press, 1996. Citado na página 15.

BIRRELL, N.; DAVIES, P. Quantum fields in curved space. Cambridge, UK: Cambridge University Press, 1982. (Cambridge Monographs on Mathematical Physics). Citado na página 21.

BOYANOVSKY, D. et al. Dynamical renormalization group resummation of finite temperature infrared divergences. Phys. Rev., D60, p. 065003, 1999. Citado na página 64.

BRAATEN, E.; PISARSKI, R. D. Deducing hard thermal loops from ward identities. Nucl. Phys., B339, p. 310-324, 1990. Citado na página 16.

BRANDT, F.; FERREIRA, R.; THUORST, J. Classical Boltzmann equation and high-temperature QED. Phys.Rev., D91, n. 4, p. 045023, 2015. Citado 3 vezes nas páginas 15, 16 e 81.

BRANDT, F. T.; CUADROS-MELGAR, B.; MACHADO, F. R. Thermal one- and two-graviton Green's functions in the temporal gauge. Phys. Rev., D67, p. 125006, 2003. Citado na página 30.

BRANDT, F. T.; DAS, A.; FRENKEL, J. Classical transport equation in noncommutative QED at high temperature. Phys. Rev., D66, p. 105012, 2002. Citado na página 88.

BRANDT, F. T.; DAS, A.; FRENKEL, J. Transport equation for the photon Wigner operator in non- commutative QED. Phys. Rev., D68, p. 085010, 2003. Citado 2 vezes nas páginas 88 e 114.

BRANDT, F. T. et al. Transport equation and hard thermal loops in noncommutative Yang-Mills theory. Phys. Rev., D66, p. 045011, 2002. Citado 2 vezes nas páginas 88 e 114.

BRANDT, F. T.; FRENKEL, J.; MACHADO, F. R. Thermal forward scattering amplitudes in temporal gauges. Published in Osaka 2000, High Energy Physics, vol. 2 1353-1355, p. 4, 2000. Citado na página 30.

BRANDT, F. T.; FRENKEL, J.; MCKEON, D. G. C. Hard thermal effects in noncommutative U(N) Yang-Mills theory. Phys. Rev., D65, p. 125029, 2002. Citado na página 88.

BRANDT, F. T.; FRENKEL, J.; TAYLOR, J. C. High temperature QCD and the classical Boltzmann equation in curved space-time. Nucl. Phys., B437, p. 433-446, 1995. Citado na página 15.

BRANDT, F. T.; FRENKEL, J.; TAYLOR, J. C. High temperature QCD and the classical Boltzmann equation in curved space-time. Nucl. Phys., B437, p. 433-446, 1995. Citado na página 81. 
BRANDT, F. T. et al. Effective actions for Braaten-Pisarski resummation. Can. J. Phys., v. 71, p. 219-226, 1993. Citado na página 64.

CHERN, S.-S.; SIMONS, J. Characteristic forms and geometric invariants. Annals Math., v. 99, p. 48-69, 1974. Citado na página 92.

CZAJKA, A.; MRICZYSKI, S. Universality of the hard-loop action. Phys.Rev., D91, n. 2, p. 025013, 2015. Citado na página 76.

DAS, A. Finite Temperature Field Theory. NY: World Scientific, 1997. Citado na página 15.

DESER, S.; GRIGUOLO, L.; SEMINARA, D. Effective QED actions: Representations, gauge invariance, anomalies and mass expansions. Phys. Rev., D57, p. 7444-7459, 1998. Citado na página 81.

DESER, S.; JACKIW, R.; TEMPLETON, S. Topologically massive gauge theories. Ann. Phys., v. 140, p. 372-411, 1982. Citado na página 92.

FADDEEV, L. D.; POPOV, V. N. Feynman diagrams for the Yang-Mills field. Phys. Lett., B25, p. 29-30, 1967. Citado 2 vezes nas páginas 16 e 48.

FEYNMAN, R. P.; HIBBS, A. R. Quantum Mechanics and Path Integrals. [S.1.]:

McGraw-Hill, 1965. Citado na página 18.

FRENKEL, J.; TAYLOR, J. C. High Temperature Limit of Thermal QCD. Nucl. Phys., B334, p. 199, 1990. Citado 2 vezes nas páginas 16 e 81.

GRADSHTEYN, I. S.; RYZHIK, M. Tables of Integral Series and Products. New York: Academic, 1980. Citado 2 vezes nas páginas 62 e 69.

HSIEH, A.; YEHUDAI, E. HIP: Symbolic high-energy physics calculations. Comput. Phys., v. 6, p. 253-261, 1992. Citado 2 vezes nas páginas 82 e 117.

KAPUSTA, J.; GALE, C. Finite-temperature field theory: Principles and applications. [S.1.: s.n.], 2006. Citado 4 vezes nas páginas 15, 20, 28 e 66.

KELLY, P. F. et al. Classical transport theory and hard thermal loops in the quark-gluon plasma. Phys. Rev., D50, p. 4209-4218, 1994. Citado na página 81.

KRAMMER, U.; REBHAN, A. K.; SCHULZ, H. Resummations in hot scalar electrodynamics. Ann. Phys., v. 238, p. 286-331, 1995. Citado na página 64.

KREUZER, M.; REBHAN, A.; SCHULZ, H. Resummed loop expansion for high temperature QCD. Phys. Lett., B244, p. 58, 1990. Citado na página 64.

LITIM, D. F.; MANUEL, C. Semi-classical transport theory for non-Abelian plasmas. Phys. Rept., v. 364, p. 451-539, 2002. Citado 2 vezes nas páginas 15 e 73.

MATSUBARA, T. A New Approach to Quantum Statistical Mechanics. Prog. Theor. Phys., v. 14, p. 351-378, 1955. Citado na página 15.

MUTA, T. Foundations of Quantum Chromodynamics. Singapore: World Scientific, 1987. Citado 3 vezes nas páginas 21, 23 e 45 . 
PESKIN, M. E.; SCHROEDER, D. V. An Introduction To Quantum Field Theory (Frontiers in Physics). Boulder: Westview Press, 1995. Citado 7 vezes nas páginas 16, 20, $23,25,28,50$ e 61.

PISARSKI, R. D. Scattering amplitudes in hot gauge theories. Phys. Rev. Lett., v. 63, p. 1129, 1989. Citado na página 62.

RAMOnd, P. Field Theory: A Modern Primer. Menlo Park: Addison-Wesley, 1990. Citado 2 vezes nas páginas 21 e 23.

SCHWINGER, J. S. Brownian motion of a quantum oscillator. J. Math. Phys., v. 2, p. 407-432, 1961. Citado na página 17.

TAYLOR, J. C.; WONG, S. M. H. The effective action of hard thermal loops in QCD. Nucl. Phys., B346, p. 115-128, 1990. Citado na página 16.

UMEZAWA, H.; MATSUMOTO, H.; TACHIKI, M. THERMO FIELD DYNAMICS AND CONDENSED STATES. 1982. Citado na página 17.

UTIYAMA, R. Invariant theoretical interpretation of interaction. Physical Review, APS, v. 101, n. 5, p. 1597, 1956. Citado na página 41.

WARD, J. C. An identity in quantum electrodynamics. Phys. Rev., v. 78, p. 182, 1950. Citado 2 vezes nas páginas 50 e 66.

WIT, B. de; FREEDMAN, D. Z. Systematics of higher-spin gauge fields. Physical Review D, APS, v. 21, n. 2, p. 358, 1980. Citado na página 79.

WONG, S. K. Field and particle equations for the classical Yang-Mills field and particles with isotopic spin. Nuovo Cim., A65, p. 689-694, 1970. Citado na página 73.

YANG, C.-N.; MILLS, R. L. Conservation of isotopic spin and isotopic gauge invariance. Phys. Rev., v. 96, p. 191-195, 1954. Citado 3 vezes nas páginas 16, 41 e 42.

ZEE, A. Quantum Field Theory in a Nutshell. Princeton: Princeton University Press, 2003. Citado na página 21. 